Albert Peters

Ökonomische

Kriterien für eine

Aufgabenverteilung in
der Marktwirtschaft

Eine deskriptive und normative Betrachtung für den Allokationsbereich 


\section{Albert Peters}

\section{Ökonomische Kriterien für eine Aufgabenverteilung in der Marktwirtschaft}

Die für marktwirtschaftliche Systeme grundlegende Frage nach der Abgrenzung öffentlicher von privaten Aufgaben wird in der Wirtschafts- und speziell der Finanzwissenschaft seit jeher kontrovers diskutiert. Der Verfasser unternimmt zunächst den Versuch, mit Hilfe verschiedener Kriterien die tatsächliche Aufgabenverteilung in der Bundesrepublik zu beschreiben und die Tauglichkeit der Kriterien für eine Abgrenzung öffentlicher Aufgaben zu überprüfen (deskriptiver Ansatz). Ziel des Hauptteils der Untersuchung ist es, auf Grundlage der Theorie der Allokationspolitik ökonomische Kriterien für eine zweckmäßige Aufgabenverteilung in der Marktwirtschaft zu entwickeln (normativer Ansatz).

Albert Peters wurde 1953 in Emden geboren. 1973-1978 Studium der Volkswirtschaftslehre an der Universität Hamburg; 1979-1981 Forschungsreferent am Forschungsinstitut für öffentliche Verwaltung bei der Hochschule für Verwaltungswissenschaften Speyer; seit 1981 wissenschaftlicher Assistent am Lehrstuhlvon Prof. Dr. K. Littmann an derHochschulefürVerwaltungswissenschaften Speyer. 
Ökonomische Kriterien für eine Aufgabenverteilung in der Marktwirtschaft 


\section{FINANZWISSENSCHAFTLICHE SCHRIFTEN}

Herausgegeben von den Professoren

Albers, Krause-Junk, Littmann, Oberhauser, Pohmer, Schmidt

Band 26

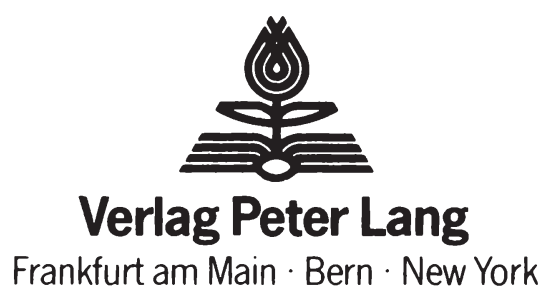




\section{Albert Peters}

\section{Ökonomische Kriterien für eine Aufgabenverteilung in der Marktwirtschaft

\author{
Eine deskriptive und normative
} \\ Betrachtung für den Allokationsbereich}

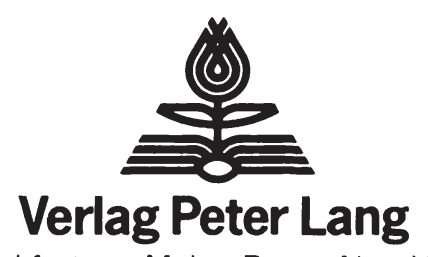

Frankfurt am Main · Bern - New York 


\section{Peters, Albert:}

Ökonomische Kriterien für eine Aufgabenverteilung in der Marktwirtschaft : e. deskriptive u. normative Betiachtung für d. Allokationsbereich / Albert Peters. - Frankfurt am Main ; Bern ; New York: Lang, 1985.

(Finanzwissenschaftliche Schriften ; Bd. 26) ISBN 3-8204-8304-7

NE: GT

Open Access: The online version of this publication is published on www.peterlang.com and www.econstor.eu under the international Creative Commons License CC-BY 4.0. Learn more on how you can use and share this work: http://creativecommons.org/licenses/by/4.0.

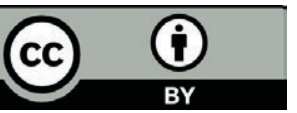

This book is available Open Access thanks to the kind support of ZBW - Leibniz-Informationszentrum Wirtschaft.

ISSN 0170-8252

ISBN 3-8204-8304-7

ISBN 978-3-631-75163-3 (eBook)

(C) Verlag Peter Lang $\mathrm{GmbH}$, Frankfurt am Main 1985

Alle Rechte vorbehalten.

Nachdruck oder Vervielfältigung, auch auszugsweise, in allen Formen wie Mikrofilm, Xerographie, Mikrofiche, Mikrocard, Offset verboten.

Druck und Bindung: Weihert-Druck GmbH, Darmstadt 


\section{VORWORT}

Die vorliegende Arbeit entstand während meiner Tätigkeit als Forschungsreferent am Forschungsinstitut für öffentliche Verwaltung bei der Hochschule für Verwaltungswissenschaften Speyer und als wissenschaftlicher Mitarbeiter am dortigen Lehrstuhl von Prof. Dr. K. Littmann. Sie wurde im Juni 1984 weitgehend abgeschlossen und im Dezember 1984 von der Hochschule als Dissertation angenommen.

Angeregt wurde die Untersuchung von meinem verehrten Lehrer, Herrn Professor Dr. Konrad Littmann. Ihm möchte ich an erster Stelle meinen aufrichtigen Dank für seine wertvolle Unterstützung und Förderung aussprechen. Herrn Professor Dr. Dieter Duwendag sei für die kritischen und ergänzenden Hinweise gedankt.

Frau Hiltrud Deubner gilt mein herzlicher Dank, da sie hilfsbereit und engagiert die Manuskripte in eine lesbare Form zu bringen verstand.

Schließlich bin ich auch den Kolleginnen und Kollegen sowie den Hörerinnen und Hörern zu Dank verpflichtet. Die wissenschaftlich äußerst fruchtbare und ausgesprochen angenehme Atmosphäre an der Hochschule, die stets Antrieb war und zugleich Abwechslung bot, hat ganz wesentlich zum Gelingen dieser Arbeit beigetragen. 
Albert Peters - 978-3-631-75163-3

Downloaded from PubFactory at 01/11/2019 07:34:44AM

via free access 


\section{$\underline{\text { Erster Teil }}$}

\section{GRUNDLAGEN}

I. Uber die Schwierigkeiten einer allgemeinen Begriffsbestimmung öffentlicher Aufgaben

1. Öffentliche Aufgaben in der rechts-, verwaltungsund politikwissenschaftlichen Diskussion

1.1. Rechtswissenschaft

1.2. Verwaltungswissenschaften

1.3. Politikwissenschaft

II. Methodische Ansätze einer Abgrenzung öffentlicher von privaten Aufgaben

2. Positiver Ansatz

3. Normativer Ansatz 


\section{DESKRIPTIVE KRITERIEN EINER ABGRENZUNG ÖFFENTLICHER} VON PRIVATEN AUFGABEN

I. Funktionale Abgrenzungskriterien

1. Bestimmung eines "öffentlichen Kernbereichs"

2. Systematisierungsversuche öffentlicher Aufgaben

2.1. Aufgabengliederungen nach formalen Kriterien

2.2. Aufgabengliederungen nach materiellen Kriterien

2. Verwirklichung des Gemeinwohls versus Maximierung des Eigennutzes

3.1. Eingrenzung der Diskussion

3.2. Zur Identifikation des Gemeinwohls in bipolaren Staatsauffassungen

3.2.1 Organische Staatsauffassung

3.2.2. Individualistische Staatsauffassung

3.3. Schlußfolgerungen

4. Erkenntniswert funktionaler Abgrenzungskriterien

II. Institutionelle Abgrenzungskriterien

1. Zur Notwendigkeit einer Überwindung der Dichotomie von Markt und Staat

2. Typologie öffentlicher und privater Aufgabenträger

2.1. Gebietskörperschaften

2.2 Parafiski

2.3. Beliehene

2.4. Unternehmen

2.5. Freie Berufe

2.6. Verbände

2.7. Selbstorganisationen

2.8. Private Haushalte 
3. Die Bedeutung der Trägerschaft als Abgrenzungskriterium $\begin{array}{ll}\text { für öffentliche und private Aufgaben } & 76\end{array}$

3.1. Die Bedeutung der Rechtsform 76

3.2. Die Bedeutung der Eigentumsverhältnisse 81

4. Die Kompetenzstruktur der Aufgabenträger 85

4.1. Informationsträger 89

4.2. Durchführungsträger 91

4.3. Finanzierungsträger 96

4.4. Entscheidungsträger 96

4.5. Aufsichtsträger 97

5. Erkenntniswert institutioneller Abgrenzungskriterien 98

III. Technisch-ökonomische Abgrenzungskriterien 99

1. Das Konzept der öffentlichen Güter 99

1.1. Konstitutive Eigenschaften öffentlicher Güter 101

1.2. Anmerkungen zum Kriterium der Nichtrivalität im Konsum 103

1.3. Anmerkungen zum Kriterium der Nichtanwendbarkeit des $\begin{array}{ll}\text { Ausschlußprinzips } & 105\end{array}$

1.4. Öffentliche Güter und öffentliche Aufgaben 108

1.5. Praktische Relevanz des Konzepts der öffentlichen Güter 109

2. Das Konzept der Koordinationsmechanismen 112

2.1. Typologie der Koordinationsmechanismen 113

2.1.1. Marktpreissystem 114

2.1.2. Windhundverfahren 115

2.1.3. Kontingentierung 116

2.1.4. Verlosung 117

2.1.5. Angebot bis zur Sättigung 118 
2.2. Alternative Typologien gesamtwirtschaftlicher

Steuerungsformen

2.3. Praktische Relevanz des Konzepts der Koordinationsmechanismen

IV. Ergebnisse

126

\section{Dritter Teil}

\section{NORMATIVE KRITERIEN FÜR EINE AUFGABENVERTEILUNG IN DER MARKTWIRTSCHAFT}

I. Zur konzeptionellen und methodologischen Trennung von Allokation und Distribution

II. Theoretischer Rahmen der allokativen Analyse

1. Das wohlfahrtsökonomische Referenzsystem

1.1. Skizzierung des wohlfahrtsökonomischen Argumentationsgerüstes

1.2. Zur wirtschaftlichen Anwendbarkeit des wohlfahrtsökonomischen Referenzsystems

2. Gesellschaftliche Ziele als Referenzsystem

3. Leitlinien eines Referenzsystems für realisierbare Alternativen

3.1. Beurteilungskriterien

3.2. Interventionsebenen und -formen

3.3. Entscheidungskriterien 
III. Kriterien für ein allokatives Marktversagen 164

1. Marktversagen auf der Angebotsseite 164

1.1. Monopolistisches Unternehmensverhalten 164

$\begin{array}{ll}\text { 1.2. Sinkende Durchschnittskosten } & 179\end{array}$

1.3. Fehlender Ausweis zukünftiger Knappheitsverhältnisse 193

1.4. Externe Effekte der Produktion 200

$\begin{array}{ll}\text { 2. Marktversagen auf der Nachfrageseite } & 217\end{array}$

2.1. Öffentliche Güter 217

2.2. Meritorische Güter 224

3. Ergebnisse 242

$\begin{array}{ll}\text { ZUSAMMENFASSUNG } & 245\end{array}$

LITERATURVERZEICHNIS 251 
AER

AöfU

BFuP

DOV

EJPR

$\mathrm{HdF}$

HdSt

HdSW

HdWW

Jb. Nat.Stat.

JEL

JLAWEC

JPE

JPOIEC

N.F.

NTJ

QJE

REStat

REStud

SchdVSocpol

SEJ

SZVS

VVDStRL

WiSta

ZfbF

ZfgS

ZögU
The American Economic Review

Archiv für öffentliche und freigemeinwirtschaftliche Unternehmen

Betriebswirtschaftliche Forschung und Praxis

Die offentliche Verwaltung

European Journal of Political Research

Handbuch der Finanzwissenschaft

Handwörterbuch der Staatswissenschaften

Handwörterbuch der Sozialwissenschaften

Handwörterbuch der Wirtschaftswissenschaft

Jahrbücher für Nationalökonomie und Statistik

The Journal of Economic Literature

The Journal of Law and Economics

The Journal of Public Economics

The Journal of Political Economy

Neue Folge

National Tax Journal

The Quarterly Journal of Economics

The Review of Economics and Statistics

The Review of Economic Studies

Schriften des Vereins für Socialpolitik

Southern Economic Journal

Schweizerische Zeitschrift für Volkswirtschaft und Statistik

Veröffentlichungen der Vereinigung Deutscher Staatsrechtslehrer

Wirtschaft und Statistik

Zeitschrift für betriebswirtschaftliche Forschung

Zeitschrift für die gesamte Staatswissenschaft

Zeitschrift für öffentliche und gemeinwirtschaftliche Unternehmen 
Verzeichnis der Obersichten

Se ite

1 Ausschnitt einer hierarchisch geordneten Ziel-(Aufgaben-) struktur für das Bildungswesen

2 Strukturebenen und Dimensionen öffentlicher Aufgaben

3 Haushaltssystematik des Bundes - Funktionenplan

4 Aufgabensystematik nach Hans Peter Bull

5 Die Institutionen der Parafiski

6 Unternehmenstypologie anhand der Unterscheidungsmerkmale Eigentumsverhältnisse und Rechtsform

7 Rechtsformen öffentlicher und privater Aufgabenträger

8 Schematische Darstellung der Kompetenzstruktur der Aufgabenträger

9 Ausschlußprinzip und Rivalität im Konsum als Kriterien zur Unterscheidung öffentlicher und privater Güter

10 Ausprägungen des Koordinationsmechanismus "Anaebot bis zur Sättigung"

11 Zusammenhang zwischen politisch-(sozio-)ökonomischen Entscheidungs- und technisch-ökonomischen Koordinationsmechanismen

12 Koordinationsmechanismen als Kriterium zur Abgrenzung öffentlicher von privater Güterbereitstellung

13 Eine grobe Matrix zur Beurteilung der Abgrenzungskriterien

14 Wohlfahrtsökonomisches Referenzsystem und öffentliche Aufgaben

15 Gesellschaftliche Ziele als Referenzsystem und öffentliche Aufgaben

16 Ein Referenzsystem für realisierbare Alternativen und öffentliche Aufgaben

17 Interventionsebenen und -formen

18 Interventionsebenen und-formen bei monopolistischem Unternehmensverhalten 
19 Interventionsebenen und -formen bei fehlendem Ausweis zukünftiger Knappheitsverhältnisse

20 Kategorien externer Effekte

21 Interventionsebenen und -formen bei externen Effekten der Produktion

22 Interventionsebenen und -formen bei verzerrten Konsumentenpräferenzen 


\section{EINFUHRUNG}

Falls es in den Wirtschafts- und in den Verwaltungswissenschaften größere Felder ungelöster Probleme gibt, so gehört zu ihnen zweifellos die Bestimmung öffentlicher Aufgaben. Bereits im Jahre 1792 formulierte zwar WILHELM VON HUMBOLDT seine "Ideen zu einem Versuch, die Grenzen der Wirksamkeit des Staates zu bestimmen"1), und einer der exponiertesten Vertreter der wirtschaftswissenschaft, JOHN MAYNARD KEYNES, schrieb 1926: "Es ist vielleicht die wichtigste Aufgabe der heutigen Nationalökonomen, von neuem zwischen den Agenda und Non-Agenda des Staates zu unterscheiden." 2) Bis heute hat aber die Diskussion über das Verhältnis zwischen Markt- und Staatswirtschaft nichts an Aktualität und Brisanz verloren ${ }^{3)}$, sondern sie wird mit unaeminderter Intensität und wechselnder StoBrichtung weitergeführt.

Einen eindeutigen Trend weisen lediglich die empirischen Fakten auf. Im Zeitablauf ist eine ständig zunehmende Aktivität des öffentlichen Sektors beobachtbar, so daB es nicht verwundert, daB das von ADOLPH WAGNER bereits 1863 aufgestellte "Gesetz der wachsenden Ausdehnung der öffentlichen und speciell der Statstäthigkeiten"4) große Beachtung gefunden hat und zumindest in der deutschen Literatur häufig den Ausgangspunkt für Untersuchungen über die Entwicklung der Staatstätigkeit bildet. Da das Wagnersche "Gesetz" jedoch den Anforderungen, die an ein Entwicklungsgesetz gestellt werden müssen, nicht gerecht zu werden vermag ${ }^{5)}$, mithin die Hypothese vom Wachstum der Staatsaktivität

1) W. von Humboldt, Ideen zu einem Versuch, die Grenzen der Wirksamkeit des Staates zu bestimmen, veröffentlicht Berl in 1841.

2) J. M. Keynes, Das Ende des Laissez-Faire, München und Leipzig 1926, S. 31.

3) "Der Fragenkreis darf daher mit Recht zu den Kardinalproblemen der Nationalökonomie gezählt werden". K. Littmann, Strukturen und Entwicklungen der staatlichen Aktivität in der Bundesrepubi ik Deutschland 1950-1970, in: Strukturwandlungen einer wachsenden Wirtschaft, hrsg. von F. Neumark, SchdVSocpol, N.F., Bd. 30/II, Berl in 1964, S. 779.

4) A. Wagner, Grundlegung der politischen Okonomie, Theil 1: Grundlagen der Volkswirtschaft, 3. Aufl., Leipzig-Heidelberg 1893, S. 895.

5) Vgl. K. Littmann, offentliche Ausgaben II: Die "Gesetze" ihrer langfristigen Entwicklung, in: HdWW, Bd. 1, Stuttaart u.a. 1977, S. $351 \mathrm{f}$. 
keinen Anspruch auf Allgemeingultigkeit erheben kann, bleibt die Frage, welche Aufgaben privat und welche öffentlich erfiult werden sollten, weiter Gegenstand heftiger Auseinandersetzungen.

Im Laufe der Zeit haben sich freilich die Akzente verschoben. Während in den 60 er Jahren und Anfang der 70 er Jahre der Ruf nach mehr öffentlichen Leistungen die politische und wissenschaftliche Landschaft in der Bundesrepublik Deutschland beherrscht hat ${ }^{1)}$, wird seit Mitte der 70 er Jahre vor allem skeptisch über Aufgabenzuwächse und einer damit möglicherweise einhergehenden oberlastung des Staates diskutiert. ${ }^{2}$ )

1) Die auf Galbraith zurückgehende These vom "privaten Wohlstand" bei gleichzeitiger "öffentlicher Armut" fand in dieser Zeit auch in der Bundesrepublik weitgehende Beachtung; vgl. Z.B. die Referate von A. Krause, W. Engels, G. Wehner und G. von Kortzfleisch, Privater Wohlstand - Offentliche Armut?, in: Der Staatssektor in der sozialen Marktwirtschaft, hrsg. von D. Duwendag, Schriftenreihe der Hochschule Speyer, Bd. 59, Berlin 1976, S. $141 \mathrm{ff}$. Zu den Möglichkeiten der Ausdehnung staatlicher Aktivitäten aus damaliger Sicht vgl. auch Wissenschaftlicher Beirat beim BMF, Gutachten zur Finanzierung eines höheren Staatsanteils am Sozialprodukt vom 3.6.1972, in: Wissenschaftlicher Beirat beim Bundesministerium der Finanzen. Entschließungen, Stellungnahmen und Gutachten 1949-1973, hrsg. vom Bundesministerium der Finanzen, Tübingen 1974, S. $535 \mathrm{ff}$. sowie K.-H. Raabe, Projektionen eines "Korridors für den Staatssektor", in: Der Staatssektor in der sozialen Marktwirtschaft, hrsg. von D. Duwendag, Schriftenreihe der Hochschule Speyer, Bd. 59, Berlin 1976, S. $37 \mathrm{ff}$.

2) Zu nennen ist hier insbesondere der Wissenschaftliche Beirat beim Bundesministerium der Finanzen, der 1975 in einem Gutachten u.a. den Abbau öffentlicher Leistungen sowie die Verlagerung bisher öffentlich angebotener Leistungen auf den privaten Bereich vorschiug und damit eine breit angelegte Privatisierungsdebatte entfachte; $v g l$. Wissenschaftlicher Beirat beim BMF, Zur Lage und Entwicklung der Staatsfinanzen in der Bundesrepublik Deutschland, Bulletin der Bundesregierung Nr. 103 vom 16.8.1975, S. $1001 \mathrm{ff}$. Es folgten Stellungnahmen u.a. vom Sachverständigenrat zur Begutachtung der gesamtwirtschaftlichen EntwickTung, Jahresgutachten 1975/76, Stuttgart-Mainz 1975, Tz. 333 ff.; von der Kommission für wirtschaftlichen und sozialen Wandel, Wirtschaftlicher und sozialer Wandel in der Bundesrepublik Deutschland, Gutachten der Kommission, Göttincen 1977, Kapitel II, Tz. 129; vom Deutschen Städtetag, Privatisierung öffent1 icher Aufgaben. DST-Beiträge zur Kommunalpolitik, Heft 2, Köln 1976; von der Gewerkschaft OTV, Zur Privatisierung öffentlicher Dienstleistungen, Stel Tungnahme des geschäftsführenden Hauptvorstandes, Heft 1, Stuttgart 1977; vom Bund der Steuerzahler (Hrsg.), Privatisierung öffentlicher Dienstleistungen, Wiesbaden 1978. VgT. auch die Dokumentation zur Privatisieruna von D. Bischoff, K.-0. Nickusch, Privatisierung öffentlicher Aufgaben, Ber1 in-New York 1977. Eine Literaturübersicht gibt $\mathrm{H}$. Hanusch (Hrsg.), Reform öffentlicher Leistungen, Baden-Baden 1978, S. $127 \mathrm{ff}$. 
Vielfältige Gründe können für diese Tendenzwende angeführt werden. GewiB ist die schnelle Zunahme der Defizite öffentlicher Haushalte mitverantwortlich für diese Entwicklung, doch nicht nur die Etatsituation, sondern der gesamte öffentliche Sektor ist in das Schußfeld der Kritik geraten. Die Schlagworte "Gesetzesflut", "Bürokratisierung" und "Staatsverdrossenheit" belegen dies eindrucksvoll. Ahnlich schlagwortartig besetzt ist aber auch die Rezeptur. Begriffe wie "Privatisierung" und "Entstaatlichung" beherrschen die oftmals durch Tagesereignisse geprägte und häufig ideologisch ausgerichtete politische Debatte.

Gegenwärtig befindet sich die politische Diskussion offenbar auf einer Privatisierungswoge der sich ablösenden "Privatisierungs-" und "Verstaatlichungs"-Wellen. 1) Die Argumentation folgt dabei in etwa diesem Ritual: Bringt der Markt unerwuinschte Ergebnisse hervor, so wird der Ruf nach staatlichen Interventionen laut. Ist hingegen der Stat an diesen unerwünschten Ergebnissen bereits beteiligt, dann wird mehr Mut zum Markt gefordert und der Sachverstand sowie die Flexibilität privater Unternehmer beschworen. Unzureichende Effizienz des öffentlichen Dienstes führt rasch zu Bestrebungen, private Dienste in Anspruch zu nehmen. Im Falle privaten MiBmanagements wird dagegen das Heil in öffentlicher Steuerung und Kontrolle gesehen. Versagt eine Verwaltung auf dezentraler Ebene, so wird Zentralisierung angestrebt. Verfügt man bereits über eine zentrale Verwaltung, ertönt vielerorts der Ruf nach mehr Bürgernähe. Die Reihe der Beispiele für solche wechselnden Argumentationsweisen ließe sich ohne Schwierigkeiten verlängern.

1) Freilich weisen allein die im Zeitablauf beobachtbaren Privatisierungsbestrebungen sehr verschiedene Facetten auf. Stand in der ersten Hälfte der 60 er Jahre die Privatisierung staatlichen Produktivvermögens im Vordergrund (Teilprivatisierung von Preussag, VEBA und VW), so rückte Mitte der $60 \mathrm{er}$ Jahre vor allem die Delegation von "hoheitlichen" Verwaltunasaufgaben auf Private (z.B. TOV) ins Blickfeld des Interesses. Gegenstand der Mitte der 70er Jahre einsetzenden und bis heute anhaltenden "großen" Privatisierunasdebatte ist dagegen vornehmlich die Obertragung der Durchführung von Versorgungsaufgaben (z.B. Muillabfuhr, Personennahverkehr) und sog. Annex-Aufgaben (z.B. Gebäudereinigung, Instandhaltung öffentlicher Fuhrparks) auf private Unternehmen. Schließlich wird in jüngster Zeit wieder über eine Neukonzeption der Beteiligungspolitik des Bundes nachgedacht. (Zu Beginn des Jahres 1984 reduzierte der Bund seine Beteiligung an der VEBA auf 30 v.H., die Veräußerung weiterer staatlicher Beteiligungen wird gegenwärtig diskutiert). 
Die wissenschaftliche Diskussion zeigt, daß es bisher an einer systematischen Bearbeitung der Probleme mangelt und statt dessen am Einzelfall darzulegen versucht wird, ob und warum eine bestimmte Aktivität (z.B. Muillabfuhr, Personennahverkehr, Hochschulbildung) als öffentliche Aufgabe zu betrachten ist und in welcher Organisationsform oder mit welchen Instrumenten diese öffentliche Aufgabe am besten durchgeführt werden kann. Dabei wird eine Vielzahl von Argumenten aus unterschiedlichsten Begründungszusammenhängen vorgetragen ( $z$.B. der Gesichtspunkt der historisch gewachsenen Ordnung, der MaBstab der Kostenminimierung, der unbestimmte Rechtsbegriff des Gemeinwohls), die als Beurteilungskriterien bestenfalls plausibel, aber in der Regel nicht zwingend aus einem System heraus abgeleitet sind. Folglich weichen auch die Argumentationsketten bei einschlägigen Untersuchungen im allgemeinen stark voneinander ab. Daraus lassen sich zum Teil auch die bemerkenswerten Differenzen in der wissenschaftlichen Beurteilung des aktuellen Streites iber eine Entstaatlichung bzw. Privatisierung öffentlicher Aufaaben erklären.

Diese - für Wissenschaft und Praxis gleichermaBen unbefriedigende - Situation führt zu dem Wunsch, grundsätzliche oberlegungen zu einer Grenzziehung zwischen öffentlichen und privaten Aufgaben vorzunehmen. Voraussetzung für eine rationale Diskussion über die Festlegung politischer Prioritäten für die Erfüllung neuer oder den Abbau vorhandener öffentlicher Aufgaben ist die Entwicklung von Kriterien, die zur Begründung der Aufgabenwahrnehmung durch den öffentlichen Sektor herangezogen werden können. An der Erarbeitung solcher Kriterien sind Vertreter verschiedener Wissenschaften zu beteiligen.

Bei den öffentlichen Aufgaben handelt es sich um ein äuBerst komplexes Gebilde, das sich dem Betrachter nur in Ausschnitten erschließt. Im grundlegenden ersten Teil der Untersuchuna werden deshalb zunächst die Konturen der wichtigsten Definitionen öffentlicher Aufgaben, die in den einschlägigen wissenschaftlichen Beiträgen auBerhalb der ökonomischen Arbeiten zu finden sind, skizziert. Dabei soll lediglich ein Eindruck von dem ty- 
pischen Vorgehen vermittelt werden, ohne die Nuancen zu markieren, die innerhalb der einzelnen Disziplinen die Auffassuna der jeweiligen Autoren voneinander trennen. Die Schwierigkeiten einer allgemeinen Begriffsbestimmung öffentlicher Aufgaben werden anschließend präzisiert. AuBerdem wird kurz auf die grundsätz1 ich unterscheidbaren methodischen Ansätze einer Abgrenzung öffentlicher von privaten Aufgaben eingegangen.

Sämtliche Versuche, die in der Bundesrepublik Deutschland gegenwärtig als öffentliche Aufgaben betrachteten Aktivitäten mit Hilfe eines einzigen umfassenden Kriteriums von privaten Aufgaben abzugrenzen, müssen an der Komplexität öffentlicher Aufgaben scheitern. Im zweiten Teil wird daher von vornherein ein anderer Weg eingeschlagen: Unterschiedliche, auf verschiedenen Ebenen liegende Kriterien werden zur Beschreibung der tatsächlichen Aufgabenverteilung in der Bundesrepublik Deutschland herangezogen (deskriptiver Ansatz). Darin eingeschlossen ist die kritische Oberprüfung des Erkenntniswerts traditioneller Kriterien sowie die Ermittlung jener Abgrenzungskriterien, die als aussagekräftig zu qualifizieren sind. Die Erörterung soll zudem einen Beitrag zur terminologischen Klarstellung und zur Systematisierung der mit uneinheitlichen Begriffen und aus unklaren Begründungszusammenhängen geführten Privatisierungsdebatte leisten. Schlieblich erleichtert sie auch den Zugang zur nachfolgenden Analyse, insbesondere ermöglicht sie die Vorstrukturierung der dort $z u$ behandelnden Fragestellungen.

Ziel des dritten Teils der Untersuchung ist der Versuch, die in der wirtschaftswissenschaftlichen Literatur diskutierten - im ökonomischen Sinne - normativen kriterien einer kritischen Prüfung zu unterziehen und, soweit erforderlich, Kriterien für eine ökonomisch zweckmäBige Aufgabenverteilung in der Marktwirtschaft zu entwickeln. Einige der auf Grundlage des wohlfahrtstheoretischen Argumentationsgerüstes abgeleiteten Fälle für ein Marktversagen können dabei als Beurteilungskriterien erneut aufgenommen werden. Mängelanalysen wurden für den allokativen Bereich vor dem Hintergrund verschiedener fundamentaler theoretischer Ansätze wie u.a. der Theorie der öffentlichen Güter (Samu- 
elson, Musgrave), der Anatomie des Marktversagens (Bator) oder dem Konzept der externen Effekte (Mishan, Head) sowie deren Weiterentwicklungen erstellt. Da diese Ansätze sich jedoch immer auf die Ableitung ausgewählter Kategorien von Marktmängeln beschränken, ist es notwendig, zusätzliche Beurteilungskriterien in das bestehende Denksystem einzufügen. Darüber hinaus ist es aber auch unerläßlich, den Argumentationsrahmen in verschiedene Richtungen zu erweitern. Dies gilt zum einen für die von der Wohlfahrtsökonomie gesetzten Totalbedingungen. Zum anderen werden aus den Idealbedingungen des allgemeinen Konkurrenzgleichgewichts die Fälle des "Marktversagens" abgeleitet und daraus häufig ohne weitere Prüfung der Schluß gezogen, durch statiliche Eingriffe sei eine effiziente Güterversorgung zu gewährleisten. Soll der Eindruck vermieden werden, die relevante Entscheidung sei diejenige zwischen einer mit Mängeln behafteten und einer ideal funktionierenden Form der Aufgabenerfüllung ("Nirwana-Ansatz"), so sind den Kosten, die der Volkswirtschaft iniolge der fehlerhaften Funktionsweise des Marktpreissystems erwachsen, die gesamtwirtschaftlichen Kosten einer Korrektur der Marktschwächen gegenüberzustellen. Dabei ist zu berücksichtigen, daß es zur Obertragung einer Aufgabe an den Staat grundsätzlich eine Reihe abgestufter Möglichkeiten gibt, die in sehr unterschiedlichem Maße Kosten verursachen, und daneben ist $z u$ bedenken, daß jeder staatliche Eingriff ebenfalls lediglich suboptimale Ergebnisse produziert. 


\section{Erster Teil}

\section{G R U N D L A G E N}

\section{Ober die Schwierigkeiten einer allgemeinen Begriffsbestim- mung öffentlicher Aufgaben}

Die umfangreiche wissenschaftliche Beschäftigung mit dem Problemkreis öffentlicher Aufgaben hat bisher wenig zur allgemeinen Begriffsbestimmung beigetragen. Vielmehr ist im Zusammenhang mit den öffentlichen Aufgaben immer noch ein babylonisches Sprachgewirr zu konstatieren, welches einerseits darauf zurückzuführen sein dürte, daß verschiedene wissenschaftliche Disziplinen sich mit diesem Fragenkreis auseinandersetzen und andererseits darauf, daß der Aufgabenbegriff selbst äußerst weit gespannt ist. Die (öffentlichen) Aufgaben stellen eine amorphe Masse dar, die sich immer nur ausschnittweise ins Blickfeld rücken läßt. Der Betrachter wird aber recht bald überfordert, wollte er den Versuch machen, alle Ecken und Winkel dieses unförmigen Gebildes zugleich auszuleuchten.

1. Offentliche Aufgaben in der rechts-, verwaltungs- und politikwissenschaftlichen Diskussion

Der überwiegend ohne jegliche Erläuterung verwendete Terminus "öffentliche Aufgaben" legt die Vermutung nahe, hier handele es sich um einen Kommunikationsbegriff, über dessen Vorstellungsinhalt breites Einverständnis angenommen werden kann und der deshalb einer klaren begrifflichen Festlegung nicht mehr bedarf. 1) Bei genauerer Betrachtung wird allerdings deutlich, daß "öffentliche Aufgaben" lediglich die klammer bilden für die Vielzahl der z.B. in rechts-, verwaltungs-, politik- und wirtschaftswissenschaftlichen Analysen verwendeten Ausdrücke wie u.a. Staats-

1) So auch E. Mäding, Aufgaben als Reformthema, in: Reform kommunaler Aufgaben, hrsg. vom Institut für Kommunalwissenschaften, Studien zur Kommunalpolitik, Bd. 19, Bonn 1978, S. 3. 
aufgaben, Verwaltungsaufgaben, öffentliche Güter, meritorische Güter, Kollektivgüter, öffentliche Leistungen oder öffentliche Bedürnisse. Zum Teil wird den genannten Begriffen ledialich der Charakter von Synonymen zugewiesen, überwiegend dient ihre Abgrenzung aber nicht nur einem sprachlichen, sondern einem sachlichen Zweck. Gilt Letzteres, so spiegelt sich in der terminologischen Variationsbreite vor allem auch das unterschiedliche Erkenntnisinteresse der beteiligten Wissenschaften wider. Es ist daher aufschlußreich, zunächst eine kurze Skizzieruna der Aufgabendiskussion in den der Wirtschaftswissenschaft benachbarten Disziplinen vorzunehmen.

\subsection{Rechtswissenschaft}

Offentliche Aufgaben werden in der jüngeren rechtswissenschaftlichen Literatur im allgemeinen nicht aus einer unmittelbaren Gegenüberstellung zu den privaten Aufgaben bestimmt, sondern nur mittelbar im Rahmen einer Diskussion des Verhältnisses staatlicher zu öffentlichen Aufgaben. 1) Während lange Zeit beide Begriffe undifferenziert fur ein und denselben Sachverhalt verwendet wurden oder sogar ausdrücklich eine Gleichsetzung erfuh$r^{2}{ }^{2}$, nehmen Juristen heute überwiegend eine scharfe Trennung zwischen staatlichen und öffentlichen Aufgaben vor. ${ }^{3}$ )

Der Blick in das Grundgesetz zeigt, daß nur wenige Artikel dem Staat ausdrücklich bestimmte Aufaaben zuweisen, daß diese Aufgabenzuordnungen vornehmlich im Rahmen der Kompetenzabgrenzung zwischen Bund und Ländern vorgenommen werden und es sich darüber hinaus um erst im Zeitablauf eingefügte Artikel handelt. ${ }^{4}$ Ex-

1) Vgl. hierzu auch den überblick über den rechtswissenschaftlichen Diskussionsstand bei $W$. Kirberger, Staatsentlastung durch private Verbände, Schriften zur öffentlichen Verwaltung und öffentlichen Wirtschaft, Bd. 30 , Baden-Baden 1978, S. $56 \mathrm{ff}$.

2) So z.B. E. R. Huber, Wirtschaftsverwaltungsrecht, Bd. 1, 2. Auf1., Tübingen 1953, S. 535 .

3) Vgl. insbes. H. Peters, offentliche und staatliche Aufqaben, in: Festschrift für H. C. Nipperdey zum 70. Geburtstag, Bd. 2, München und Berl in 1965, S. $877 \mathrm{ff.}$; vgl. daneben H. H. Klein, Zum Begriff der öffentlichen Aufgabe,

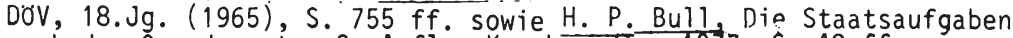
nach dem Grundgesetz, 2. Aufl., Kronbergits. 1977, S. $48 \mathrm{ff}$.

4) Vgl. H. P. Bull, a.a.0., S. 149; H. H. Klein, Zum Begriff der öffentlichen Aufgabe, a.a.0., S. $757 \mathrm{f}$. 
plizit werden im Grundgesetz lediglich folgende Funktionen als statiche Aufgaben genannt:

- Aufstellung der Streitkräfte zur Verteidigung (Art. 87a)

- Katastrophenschutz (Art. 87a, 2 i.V.m. 35, 2 und 3)

- Abwehr von Gefahren für den Bestand des Bundes oder eines Landes, Notstandsverfassung (Art. $87 a, 4$ i.V.m. 91)

- Konjunkturstabilisierung (Art. 109)

- Ausbau und Neubau von Hochschulen (Art. 91a)

- Verbesserung der Agrarstruktur und des Küstenschutzes (Art. 91a)

- Bildungsplanung (Art. 91b)

- Forschungsförderung (Art. 91b)

- Aufsicht uiber das Schulwesen (Art. 7)

- Verwaltung des Hoheitsvermögens (Art. 87, 87b, 87c, 87d, 88, $89,90)$.

Ober die namentliche Nennung dieser wenigen Statsaufgaben hinaus ist die Verfassung jedoch geprägt durch eine Reihe von Grundprinzipien, nämlich dem Rechtsstaatsprinzip, dem Sozialstaatspostulat und dem Subsidiaritätsprinzip. Mit der Formulierung dieser Rechtsnormen umschreibt die Verfassung nun nicht mehr einen fest umrissenen und geschlossenen Bereich stati icher Aktivitäten, sondern im Rahmen dieser Normen ist den Interpretationen daruber, was als statiche Aufgabe zu beareifen ist, breiter spielraum gegeben. ${ }^{1)}$

Das Grundgesetz überläßt es also in hohem Maße dem Gesetzgeber selbst, die notwendige Konkretisierung der Normen vorzunehmen, wobei der Staat diese Kompetenz-Kompetenz nach RUPERT SCHOLZ bisher zu einer extensiven Ausdehnung der Staatsaufgaben genutzt hat. 2) Damit bleibt aber festzustellen, daß das Grund-

1) Zu den verschiedenen Interpretationen des Sozialstaatsprinzips sowie deren - durch das Rechtsstaats - und Subsidiaritätsprinzip gezogenen - Grenzen siehe H.P. Bull, a.a.0., S. $167 \mathrm{ff}$.

2) $\mathrm{Vgl}$. R. Scholz, Grenzen staatlicher Aktivität unter der arundaesetzlichen Wirtschaftsverfassuna, in: Der Staatssektor in der sozialen Marktwirtschaft, hrsg. von D. Duwendag, Schriftenreihe der Hochschule Spever, Bd. 59, Berlin 1976, S. 120. 
gesetz kaum weiterhelfen kann, wenn es gilt, die Aufgaben des Staates zu bestimmen. Die wenigen expliziten Aufqabenzuweisungen folgen offensichtlich keinem übergeordneten gemeinsamen Kriterium, und die Grundprinzipien der Verfassung sind zu unpräzise, um eine klare Abgrenzung zu ermöalichen. Schließlich bleibt auch die Frage nach der Trennungslinie zwischen staatlichen und öffentlichen Aufgaben offen, da das Grundgesetz hier keine begriffliche Unterscheidung vornimmt.

Dieses Ergebnis wird unterstrichen durch die Rechtsprechung oberster Gerichte. Auch hier mangelt es an direkten Stellungnahmen zur Abgrenzung privater und öffentlicher Aufgaben. Dort, wo die Verfassungsrichter darüber zu befinden hatten, in welchen Fällen eine staatliche im Gegensatz zu einer öffentlichen Aufgabe vorliegt, lassen die Urteilsbegründungen vermuten, daß die Richter ihre Entscheidungen in jedem der Fälle auf Grund anderer Argumente getroffen haben. ${ }^{1)}$ Nicht selten entsteht auch der Eindruck, die Urteile seien nachträgliche Rechtfertigungen historisch gewachsener Zustände. ${ }^{2)}$ Ein gemeinsames Kriterium für die Unterscheidung staticher und öffentlicher Aufaaben fehlt auch hier. Offenbar gibt es aber eine Vielzahl von Aufgaben, die, obwohl sie nicht direkt vom Staat erfüllt werden, so doch "öffentliche Bezüge" aufweisen. Offentliche Aufgaben sind deshalb für HANS PETERS solche, "an deren Erfüllung die offentlichkeit maßgeblich interessiert ist." 3 ) Das Vorliegen eines öffentlichen Interesses ist jedoch für ihn noch nicht konstitutiv für die obernahme der Aufgabe durch den Staat. Vielmehr können auch Private zur Erfüllung öffentlicher Aufgaben tätig werden. Demzufolge stellen die durch statliche Erfüllung gekennzeichneten Staatsaufgaben eine Teilmenge des größeren Bereichs der öffentIichen Aufgaben dar. ${ }^{4)}$ Das öffentliche Interesse wird damit mit-

1) So wird Z.B. die ärztliche Betreuung der Bevölkerung als staatliche Aufaabe (BVerfGE 6, S. 252), die Tätigkeit des Kassenarztes hingegen als öffentliche Aufgabe angesehen (BVerfGE 11, S. 39). Weitere Beispiele finden sich bei M. Krautzberger, Die Erfüllung öffentlicher Aufgaben durch Private, Berlin 1971, S. $97 \mathrm{ff}$.

2) Vgl. 2.B. die Urteilsbegründung zum Arbeitsvermittlungsmonopol des Staates, BVerfGE 21, S. $254 \mathrm{ff}$.

3) H. Peters, a.a.0., S. 878.

4) Ebenda, S. 879. 
telbar auch zum Abgrenzungskriterium zwischen öffentlichem und privatem Aufgabenbereich, da nach der obigen Definition immer dann eine Aufgabe - quasi als Restgröße - als privat identifiziert werden kann, wenn die offentlichkeit einer bestimmten Tätigkeit ihr maßgebliches Interesse versagt.

Wird der Versuch unternommen, die bisher offen gelassenen materiellen Merkmale des öffentlichen Interesses inhaltich zu bestimmen, ergeben sich freilich immense Schwieriakeiten. Gute Gründe sprächen nämlich dafür, daß dann z.B. der Brötchenverkauf des Bäckers als öffentliche Aufgabe zu betrachten wäre, dokumentieren doch die von ihm zu erfüllenden Auflagen wie Mindestaewicht der Brötchen und Nachtbackverbot ein weitgehendes Interesse der offentlichkeit an seiner Arbeit. Selbst eine so private Tätigkeit wie die des Friseurs mag dann zum Gegenstand des öffentlichen Interesses werden, da er doch zur Preisauszeichnung und zur Erfüllung von Mindesthygienestandards verpflichtet ist. Bei hinreichend weiter Interpretation des Kriteriums "offentiches Interesse" kann somit letztlich jede private Aktivität zur öffentlichen Aufgabe erhoben werden. Die Diskussion um das öffentiche Interesse hat deshalb bereits FORSTHOFF dazu veranlaßt, "die konkrete Entscheidung des Staates darüber, was öffentlich und was privat ist" ${ }^{1)}$ zum einzig sinnvollen Abgrenzungskriterium zu erheben. Damit wird jedoch erneut lediglich auf die Gestaltungskompetenz des Gesetzgebers verwiesen. 2) Für den um Legaldefinitionen bemühten Juristen ist vielleicht auf diesem Wege eine befriedigende A.ntwort gefunden. Für den okonomen, dessen Erkenntnisinteresse in der Aufdeckung vor allem funktionaler Zusammenhänge liegt, nimmt die Aussage, daß alles das öffentliche Aufgabe sei, was der Staat zur öffentlichen Aufgabe erklärt, eher den Charakter einer Leerformel an.

1) E. Forsthoff; Die öffentliche Körperschaft im Bundesstaat, Beiträge zum öffentTichen Recht der Gegenwart, Bd. 3, Tübing̣en 1931, S. 17.

2) $\mathrm{Vgl}$. dazu in der jüngeren Literatur Mäding, der darauf hinweist, daß "die Bestimmung des öffentlichen Interesses ... im Rahmen des verfaßten staatlich-gesellschaftlichen Gesamtsystems konkret entschieden wird." E. Mäding, Aufgaben als Reformthema, a.a.0., S. 13. So auch F. Ossenbühl, Die Erfüllung von Verwaltungsaufgaben durch Private, VVDStRL, Bd. 29, Berl in 1971, S. 153 m.w.N. 
Hingegen zeigt die in der Rechtswissenschaft vorherrschende Trennung von staatiichen und öffentlichen Aufgaben und der damit verbundene Hinweis auf die unterschiedliche Trägerschaft öffentiicher Aufgaben den Weg für die weitere Untersuchung. Zu überprüfen ist, ob die in der Rechtswissenschaft dominierende Meinung, Statsaufgaben seien eine Teilmenge der öffentlichen Aufgaben, unter Berïcksichtigung ökonomischer Aspekte zumindest in Zweifel gezogen, wenn nicht gar revidiert werden muß und es statt dessen vielmehr zutrifft, daß öffentliche Aufgaben sowohl vom Staat als auch von den Privaten erfültt werden, ebenso wie private Aufgaben nicht nur Privaten, sondern auch dem Staat überlassen sein können.

\subsection{Verwaltungswissenschaften}

Ennerhalo der Verwaltungswisserischaften weruen die Komplexität öffentlicher Aufgaben und die damit einhergehenden Probleme einer Begriffsbestimmung ausdrücklich und übereinstimmend herausgestellt. Während aber einzelne Autoren die Notwendigkeit der Bestimmung von Kriterien für eine Aufgabenabgrenzung als Grundlage einer - noch zu entwickelnden - Theorie der öffentlichen Aufgaben betonen ${ }^{1)}$, wird von anderen darauf hingewiesen, daß die Indeterminiertheit und zeitlich-räumliche Bedingtheit öffentlicher Aufgaben aus verwaltungswissenschaftlicher Sicht zu dem Ergebnis führt, "daß es allgemeingültige Kriterien für die Bestimmung öffentlicher Aufgaben nicht gibt."2) Mit dem Versuch, den vermeintlich engeren Terminus "Verwaltungsaufgaben" 3 ) mittels eines inhärenten kriteriums zu umschreiben, scheint ebenfalls wenig gewonnen, stellt doch LUHMANN dazu resümierend fest:

1) Vgl. etwa E. Mäding, Aufgaben als Reformthema, a.a.0., S. 4 f.; R. Hill-

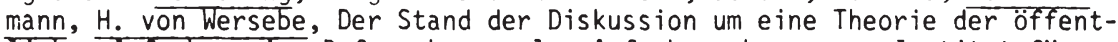
Tichen Aufgaben, in: Reform kommunaler Aufgaben, hrsg. vom Institut für Kommunalwissenschaften, Studien zur Kommunalpolitik, Bd. 19, Bonn 1978, S. 65.

2) F. Wagener, Organisation der öffentlichen Verwaltung, in: Handwörterbuch der Organisation, 2. Auf1., Stuttgart 1980, Sp. 1407; vgl. auch E. Laux, Aufgabenentwicklung und Personalbedarf in der öffentlichen Verwaltung, DOV, 32. Jg. (1979), S. 729.

3) Vgl. Z.B. E. Becker, Verwaltungsaufgaben, in: Verwaltung, hrsq. von F. Morstein-Marx, Berl in 1965, S. $187 \mathrm{ff}$.; E. Mäding, Aufgaben der öffentlichen Verwaltung, in: Die Verwaltung, Bd. б (1973), S. $257 \mathrm{ff}$. 
"Ein Verwaltungsbegriff, der die gesamten Einzelhandlungen der nicht-regierenden Exekutive deskriptiv bezeichnen könnte, hat sich bisher nicht finden lassen. Die Suche danach sollte einaestellt werden. Die Tätigkeiten sind so heterogen, daß ihr Allaemeinbegriff ins Nichtssagende verdünnt werden müBte und dadurch unspezifisch würde."1) Die Verwaltungswissenschaften behelfen sich deshalb bei der Beantwortung der Frage nach der Bestimmung öffentlicher Aufgaben mit dem Verweis auf den politischen EntscheidungsprozeB, d.h. Sie gehen davon aus, daß politische Handlungsträger den Kreis der öffentlichen Aufgaben festsetzen und ihnen hierbei ein breiter Spielraum für alternative Formen der Aufgabenzuweisung verbleibt. ${ }^{2}$ ) Demzufolge geht die verwaltungswissenschaftliche Literatur überwiegend pragmatisch vor, indem sie formale, äußerlich erkennbare und an dem jeweiligen objekt der Untersuchung ausgerichtete Abgrenzungskriterien verwendet. Untersuchungsgegenstand ist hier vor allem die Verteiluna öffentlicher Aufgaben zwischen oder innerhalb administrativer Teilsysteme, etwa

- Aspekte einer zweckmäßigen Aufgabenzuweisuna auf die Haupteinheiten der öffentlichen Verwaltung (Bund, Länder, Kreise, Gemeinden) sowie Reformen bestehender Aufgabenverteilungen, 3 )

- Ansätze und Instrumente einer Aufgabenüberprüfung in speziellen Verwaltungen, 4 )

1) N. Luhmann, Theorie der Verwaltungswissenschaft, Bestandsaufnahme und Entwurf, Köln und Berlin 1966, S. 71.

2) "In einem Staatswesen westlicher Prägung werden jeweils die Aufgabenbereiche als öffentliche Aufgaben angesehen, die die politischen Vertreterkörperschaften in einem bestimmten Zeitpunkt und für ein bestimmtes Gebiet im Rahmen einer bestimmten sozialen, gesellschaftlichen und wirtschaftlichen Lage durch die Aufnahme in den Haushaltsplan oder anderswie zur öffentlichen Aufgabe erklärt haben." F. Wagener, Organisation der öffentlichen Verwaltung, a.a.0., Sp. 1407 (Unterstreichung im Original kursiv).

3) Vgl. Z.B. Institut für Kommunalwissenschaften (Hrsq.), Reform kommunaler Aufgaben, Studien zur Kommunalpolitik, Bd. 19, Bonn 1978.

4) Vgl. z.B. R. Dieckmann, Aufgabenkritik in einer Großstadtverwaltuna, Schriftenreithe der Hochschule Speyer, Bd. 65, Berlin 1977. 
- Möglichkeiten einer Obertragung öffentlicher Aufgaben an organisatorisch und/oder rechtlich verselbständigte Aufgabenträger. 1)

Zur Abgrenzung öffentlicher Aufgaben wählt dabei z.B. DIECKMANN das Kriterium Trägerschaft. Nach seiner Auffassung sind alle jene Aufgaben als öffentliche zu bezeichnen, die zum Zeitpunkt der Oberprüfung von öffentlichen Trägern wahrgenommen werden. Zur näheren Spezifizierung der eher vagen Formulierung "Wahrnehmung öffentlicher Aufgaben" werden dann der Einsatz öffentlichen Personals und öffentlicher Mittel herangezogen. 2 )

Die nur skizzenhaften Ausführungen zur Vorgehensweise der Verwaltungswissenschaften lassen erkennen, daß es - auch aus verwaltungswissenschaftlicher Sicht - kein umfassendes inhaltliches Kriterium zur Abgrenzung öffentlicher und privater Aufgaben gibt. Eine Abgrenzung kann allenfalls mit Hilfe verschiedener formaler Kriterien, die am jeweiligen Untersuchungsgegenstand ausaerichtet sind, vorgenommen werden. Gleichzeitig unterstreicht auch die verwaltungswissenschaftliche Literatur die Bedeutung einer exakteren Analyse der Aufgabenträger, da offenbar öffentliche Aufgaben in höchst unterschiedlichen Organisationsformen vollzogen werden.

\subsection{Politikwissenschaft}

Die Rechtswissenschaft und die Verwaltungswissenschaften überlassen die Bestimmung öffentlicher Aufgaben also letztlich dem politischen Entscheidungsprozeß. Daraus könnte der Schluß gezogen werden, $d a B$ eine Abgrenzung öffentlicher und privater Aufgaben insbesondere das Erkenntnisinteresse der Politikwissenschaft gefunden hat und $d a B$ die Bemühungen um eine begriffliche Bestimmung

1) Vgl. F. Wagener, Typen der verselbständigten Erfuillung öffentlicher Aufgaben, in: Verselbständigung von Verwaltungsaufgaben, hrsg. v. F. Wagener, Schriften der Deutschen Sektion des Internationalen Instituts für Verwaltungswissenschaften, Bd. 1, Bonn 1976, S. 31 ff.; G. F. Schuppert, Die Erfüllung öffentlicher Aufgaben durch verselbständigte Verwattungseinheiten, Göttingen 1981.

2) R. Dieckmann, a.a.0., S. 72 . 
des Untersuchungsgegenstandes in dieser Wissenschaftsdisziplin besonders weit fortgeschritten sind. Ein Blick in die Literatur zeigt indes, daB auch aus politikwissenschaftlicher sicht weder eine allgemeingültige Begriffsklärung öffentlicher Aufgaben noch die Erstellung eines historisch ableitbaren Entwicklungsschemas möglich ist. "Was vorhanden ist, ist irgendwann einmal unter sehr unterschiedlichen Bedingungen der öffentlichen Hand zugewachsen oder zugesprochen worden. Die meisten dieser Bedingungen lassen sich im nachhinein feststellen. Der Bestand an sich bleibt dennoch ein Zufallsgebilde. Eine leitende Idee wird man nur schwerlich entdecken."1) Deshalb verweist auch die Politikwissenschaft selbst auf den politischen ProzeB, denn ELLWEIN stellt fest: "Was aus welchem Grund öffentliche Aufgabe zu sein hat, muß sich aus den Verhältnissen und dem jeweiligen politischen Kräftespiel ergeben."2)

Wird systemtheoretisch argumentiert, so ergeben sich grundsätzlich zwei mögliche Ansatzpunkte für den Versuch einer Bestimmung öffentlicher Aufgaben aus dem politischen ProzeB. Die ursprünglich von EASTON ${ }^{3}$ ) entwickelte politische systemtheorie betrachtet das politische system in erster Linie als Steuerungszentrale der Gesellschaft, in dem Forderungen und gewährte Unterstiutzung (Politik-Inputs) in politische Entscheidungen (Politik-Outputs) verwandelt und über deren Erfolg und Wirkung Rückmeldungen (Feedbacks) erstattet werden, die wiederum als politische Inputs fungieren. $\left.{ }^{4}\right)$

1) Th. Ellwein, Regierung und Verwaltung, 1. Teil: Regierung als politische Führung, Stuttgart u.a. 1970, S. 73.

2) Th. Ellwein, Einführung in die Regierungs- und Verwaltungslehre, Stuttgart u.a. T966, S. 35.

3) Vgl. D. Easton, A Framework for Political Analysis, Englewood Cliffs 1965.

4) Es ist darauf aufmerksam zu machen, daß die aus dem ökonomischen Sprachgebrauch übernommenen Begriffe Input und Output in der Politikwissenschaft eine völlig andere Bedeutung besitzen. Da sie aber inzwischen in der politischen Systemtheorie weitgehend Verwendung finden, empfiehlt sich auch hier die Beibehaltung der gewählten Terminologie. Zur besseren Unterscheidung werden sie jedoch ausdruicklich als politische Inputs und Outputs gekennzeichnet oder in Anführungszeichen gesetzt. 
Die erste Möglichkeit besteht demnach darin, die Ergebnisse des politischen Entscheidungsprozesses zu analysieren, d.h. die Phänomene zu erfassen, welche die Aktionen der politischen Handlungsträger sowie den am Ende dieses Prozesses stehenden Politik-output determinieren und damit auch die Aufgabenzuordnung zum öffentlichen und privaten Sektor politisch begründen. Eine solche Analyse zeigt insbesondere, daB aufgrund der Wirkungsweise des politischen Prozesses weder eine extensive Ausdehnung noch ein rigoroser Abbau öffentlicher Aufgabenerfiillung zu erwarten ist. Vielmehr düfte die in der Vergangenheit erfolgte Aufgabenverteilung eine wichtige Orientierungsmarke für die Politiker bilden, die gegenwärtig über eine Neuordnung der Aufgaben zu entscheiden haben, dies umso mehr, da jeder Versuch einer veränderten Aufgabenwahrnehmung erfahrungsgemäß auf den Widerstand der durch das bisher angewandte Verfahren Begünstigten stößt. Daraus erklärt sich auch, warum längst erkannte Mängel in der Aufgabenzuordnung gar nicht oder nur zögernd behoben werden und die bestehende Praxis der Aufgabenerfiullung auch dann erhalten bleibt, wenn alle anderen Erwägungen gegen einen Fortbestand in dieser Form sprechen. Die Politikwissenschaft gibt damit Erklärungen dafür, weshalb keine totale Neuordnung der Aufgaben, sondern in der Regel nur marginale Korrekturen der Aufgabenverteilung möglich sind. Sie liefert mithin Erkenntnisse über das Verhalten der Politiker, den Einfluß von Verwaltungen und Interessengruppen sowie der typischen Wirkungsweise des politischen Prozesses, die treffend gekennzeichnet wird durch Schlagworte wie "Politik der kleinen Schritte" oder "piecemeal engineering", "muddling through", "Prinzip des rekurrenten Ausschlusses", "Inkrementalismus" etc." Die Analyse des "Outputs" des politischen Prozesses ermöglicht somit zwar, die Faktoren zu benennen, welche die Realisierungschancen einer grundsätzlich veränderten Grenzziehung zwischen öffentlichem und privatem Bereich beträchtlich vermindern, und sie gibt damit bereits wichtige Hinweise auch für die Rahmenbedingungen

1) Vgl. dazu Ch. E. Lindblom, Inkrementalismus: Die Lehre vom "Sich-Durchwursteln", in: Wohlfahrtsstaat und Massenloyalität, hrsg. von K.-D. Narr und C. Offe, Köln 1975, S. $161 \mathrm{ff}$. 
der ökonomischen Untersuchung, dennoch sind auf diesem Wege keine inhaltichen Kriterien für eine Bestimmung öffentlicher Aufgaben zu gewinnen. Was politisch als öffentliche Aufgabe zu betrachten ist, bleibt weiterhin offen.

Die zweite Möglichkeit besteht darin, den Ansatzpunkt für eine Aufgabenbestimmung auf der "Input"-Seite des politischen Prozesses zu suchen. Vereinfacht können unter "Inputs" die verschiedenen Programme der politischen Parteien und die differierenden Vorstellungen der gesellschaftlichen Gruppen (Verbände, Bürgerinitiativen, Eliten etc.) einerseits sowie die dahinter stehenden kontroversen politischen Theorien andererseits verstanden werden. 1) Es bedarf keiner näheren Erläuterung, daB beide "Inputbereiche" eine Fiille sich teils unterstuitzender, teils konkurrierender Kriterien für eine Bestimmung öffentlicher Aufgaben bieten. So reicht bspw. das Spektrum der den politischen Theorien zu entnehmenden Kriterien von der Forderung nach einem weitgehenden Abbau öffentlicher Aufgaben (konservative Positionen) und Auffassungen, die dem Stat alle jene Aufgaben zuweisen, die zur Aufrechterhaltung der Funktionsfähigkeit des pluralistischen systems notwendig sind (beschreibendrechtfertigende Positionen) über polit-ökonomische Thesen, die dem Staat und dessen Aufgaben aus dem Prozeß der kapitalistischen Warenproduktion heraus erklären und daraus die Notwendigkeit der Erbringung spezifischer Steuerungs- und Komplementärleistungen durch den Staat ableiten (politische Krisentheorien) bis hin zu neo-marxistischen Positionen, in denen der Stat die allgemeinen Interessen der bürgerlichen Gesellschaft als "ideeller Gesamtkapitalist" wahrnimmt und öffentliche Aufgaben sich ergeben aus der geschichtichen Bewegung des Akkumulationsprozesses und den Krisen- und Zusammenbruchstendenzen des Kapita1 ismus. 2)

1) Ausführlicher dazu D. Easton, A Framework for Political Analysis, a.a.0., S. $12 \mathrm{ff}$.; W.-D. Narr, Theoriebegriffe und Systemtheorie, 3. Aufi., Stuttgart U.a. 1972, S. 151; W. Jann, Kategorien der Policy-Forschung, Speyerer Arbeitshefte 37, Speyer 1981, S. $23 \mathrm{ff}$.

2) Einen überblick über die grundsätzlichen Positionen der zentralen politischen Theorien geben $C$. Böhret, W. Jann, M. T. Junkers und E. Kronenwett, Innenpolitik und politische Theorie, Opladen 1979, S. $309 \mathrm{ff}$. 
Wie nicht anders zu erwarten, bietet auch die "Input"-Seite des politischen Prozesses infolge der Vielzahl äußerst heterogener politischer Kriterien für eine Identifizierung öffentlicher Aufgeben nicht die Möglichkeit einer exakten Grenzziehung zwischen öffentlichem und privatem Bereich.

\section{Zur Spannweite des Aufgabenbegriffs}

Eine systematische Erfassung öffentlicher Aufgaben wird auch durch den Umstand erschwert, daß Wort und Begriff der Aufgabe häufig ohne Erläuterung wie selbstverständlich verwendet werden und deshalb oftmals mehrdeutig und unscharf bleiben. Offenbar gehört die Aufgabe bzw. öffentliche Aufgabe "zu den handlichen Kommunikationswörtern der vorwissenschaftlichen wie der speziellen fachlichen Diskussion über Gesellschaft, Politik, Staat und öffentliche Angelegenheiten"1). Die Aufgabe vermittelt dabei von einem vielfältigen Sachverhalt eine Grundvorstellung und steht deshalb nicht selten synonym für Zweck, Ziel, Funktion, Obliegenheit, Anliegen, Bedürnis oder Pflicht. Daneben wird sie auch im Sinne von Auftrag, Denkgegenstand oder Tat verwendet. 2 )

KRAUTZBERGER betont, daß dem Wort "Aufgabe" im allgemeinen Sprachgebrauch und in der Fachsprache unterschiedliche Bedeutungsinhalte zukommen. In der Allgemeinsprache kann Aufgabe entweder "etwas Aufgegebenes" meinen und rückt so in die Nähe von "Auftrag", wobei dann insbesondere die Pflichtkomponente betont wird, oder aber unter Aufgabe wird "anvertrauen" verstanden und damit primär auf "Befugnis" abgezielt. Als Rechtsbegriff wird demgegenüber die Aufgabe zumeist mit Kompetenzzuweisung gleichgesetzt oder steht ganz allgemein für Tätigkeitsbereich im Sinne eines zugleich garantierten und limitierten Wirkungskreises einer Person oder Institution. ${ }^{3)}$ BULL stellt besonders heraus, daB Aufgaben nicht Aktivitäten um ihrer selbst willen begründen, son-

1) E. Mäding, Aufgaben der öffentlichen Verwaltung, a.a.0., S. 258.

2) Ebenda m.w.N.

3) Vgl. M. Krautzberger, a.a.0., S. $41 \mathrm{ff}$. 
dern daß mit dem Wort Aufgabe stets die deutliche Hinordnung auf ein Ziel verbunden ist. ${ }^{1)}$ Dort, wo innerhalb der Wirtschaftswissenschaft der Terminus Aufgabe explizit Verwendung findet (z.B. bei der Aufgabenplanung), wird ebenfalls auf dessen Zielorientierung hingewiesen. Wesentliches Kennzeichen einer Aufgabe ist somit also ihr Mittelcharakter im Hinblick auf die Realisierung von Zielen.

Da Ziele jedoch sowohl äußerst allgemein als auch sehr konkret formuliert werden können, bietet die Berücksichtigung des Zielaspektes allein noch keine Gewähr für eine präzisere Aufgabendefinition. Vielmehr bleibt der Aufgabenbegriff extrem weit gespannt und folglich kann auch der materielle Gehalt einer Aufgabe recht unterschiedliche Dimensionen aufweisen. So wird z.B. der weit interpretierbare Bereich "Verbesserung der Gesundheitsvorsorge" ebenso als Aufgabe formuliert wie die wesentlich konkreter umschriebene Aktivität "Ausstattung von Krankenhäusern mit modernen Röntgengeräten". Was sinnvollerweise als Aufgabe gelten soll, ist abhängig vom jeweiligen Erkenntnisinteresse bzw. vom konkreten Untersuchungsgegenstand und muB daher der fallweisen Entscheidung überlassen bleiben. Aus der Mehrdimensionalität und der Vielzahl unterschiedlicher Anwendungsfälle deshalb bereits den SchluB zu ziehen, der Aufgabenbegriff sei ungeeignet für die allgemeine fachliche Diskussion, ist jedoch zumindest verfruiht. Statt dessen sollte bei Aufgabenanalysen zunächst eine hierarchisch geordnete Ziel-bzw. Aufgabenstruktur festgelegt werden, anhand derer die dem spezifischen Erkenntnisinteresse der jeweiligen Analyse entsprechende Bedeutungsebene der Aufgabe sichtbar gemacht werden kann.

Eine solche Ziel-(Aufgaben-)hierarchie ist gekennzeichnet durch eine stufenweise Ableitung mehrerer nachgeordneter Teilziele aus zuvor ermittelten Oberzielen. Der Abstraktionsgrad der Ziele nimmt dabei mit Richtung nach unten immer weiter ab; je tiefer die Teilziele in der Hierarchie angesiedelt sind, desto konkreter ist also ihr Inhalt zu umschreiben. Diese schrittweise Auf-

1) Vgl. H. P. Bull, a.a.0., S. 44. 
fächerung zu einer Aufgabenstruktur kann übersichtich und systematisch in Form eines sich verzweigenden Ziel-Mittel-Baumes dargestellt werden. Auf der obersten Baumebene wird das generelle Ziel formuliert und auf der direkt untergeordneten Ebene werden dann alternative Mittel zur Erreichung des oben definierten Zieles erfaßt. Diese alternativen Mittel haben zugleich wieder Zielcharakter für die nachfolgende Ebene etc. Jede Ebene hat demnach zugleich Ziel- und Mittelfunktion, mit Ausnahme der obersten Ebene, die ausschließlich der Zielformulierung dient, und der untersten Ebene, die lediglich zur Beschreibung ganz spezieller Mittel herangezogen wird.

Ziel- oder Aufgabenstrukturen bildeten bereits zu Beginn der sechziger Jahre in den USA eine wesentliche Grundlage für die öffentliche Planung, und zwar im Rahmen des zunächst von McNamara im US-Verteidigungsministerium eingeführten, nach den dortigen Erfolgen auf die gesamte Bundesverwaltung ausgedehnten, später jedoch unter Präsident Nixon wieder weitgehend abgeschafften Planning-Programming-Budgeting Systems (PPBS), mit dem der Obergang von einer primär ausgabenorientierten Planung zu einer zielbezogenen und von administrativen Organisationsstrukturen losgelösten Aufgabenplanung vollzogen werden sollte. 1) Ein solchermaBen hierarchisch strukturiertes Ziel-bzw. Aufgabensystem ist das gemeinsame Ergebnis der in der Planungsphase des PPBS formulierten gesellschaftlichen Ziele (National Goals Analysis) und der Ausarbeitung einer sog. Programmstruktur - die schrittweise Operationalisierung der oberziele zu konkreten Programmielen - im Rahmen der Programmierungsphase. Die Programmstruktur, die im Regelfall drei bis vier Ziel-oder Aufgabenebenen umfaßt, besteht aus Programmkategorien und - subkategorien, Programmelementen und -subelementen (siehe ubersicht 1). Die allgemein formu-

1) Einen informativen überblick über Funktionsweise und Anwendungsprobleme des PPBS gibt D. Brümmerhoff, Das "Planning-Programming-Budgeting System" (PPBS), in: Finanzarchiv, N.F., Bd. 29 (1970), S. $64 \mathrm{ff} . ;$ zu den Zielen, Ansatzpunkten und Grenzen öffentlicher Aufgabenplanung vgl. in diesem Zusammenhang auch E. Wille, Art. Offentlicher Haushalt IV: Finanz- und Aufgabenplanung, in: HdWW, Bd. 5, Stuttgart u.a. 1980, S. 605 ff. 


\section{Übersicht 1:}

Zielebene

Programm-

kategorien

(Leitbilder)

Programm-

subkategorien

(Funktionen)

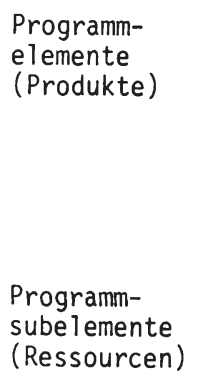

\section{Ausschnitt einer hierarchisch geordneten Ziel-(Aufgaben-) struktur}

Erhöhung des Bildungsniveaus der Bevölkerung

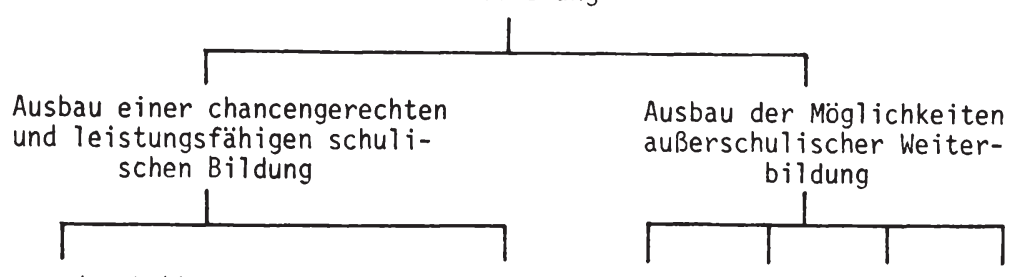

Steigerung der Zahl der Abschluisse an weiterbildenden Einrichtungen

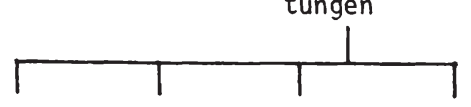

differen- Einrich- Bildungs- individuaziertes tung von beratung lisierter Bildungs- Förderangebot kursen

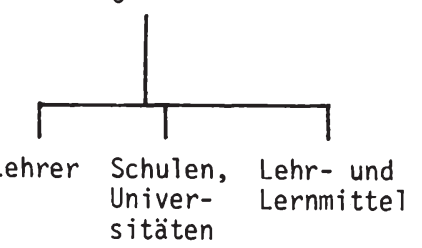

Steigerung der Zahl der Absolventen aus sozial benachteiligten Schichten

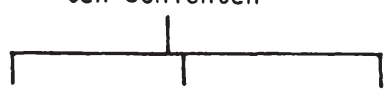

Erhöhung Begabtender Durch-mobilisie- finanzielle lässigkeit rung und Unterstuitzung des Bil- -förderung dungssys tems
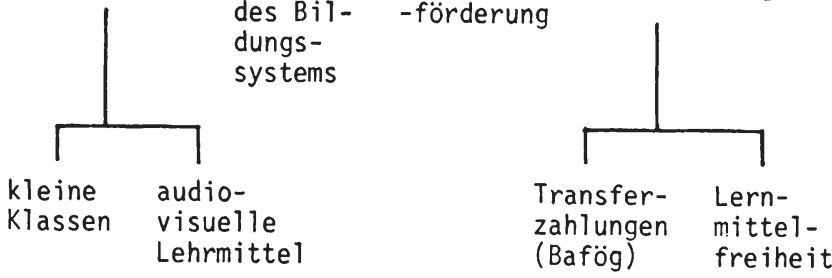
lierten gesellschaftlichen Ziele (z.B. Erhöhung des Bildungsniveaus der Bevölkerung) erfahren durch die auf der obersten Strukturebene angesiedelten Programmategorien eine erste, wenn auch noch sehr vage inhaltliche Umschreibung (z.B. Ausbau einer chancengerechten und leistungsfähigen schulischen Bildung). Mit $\mathrm{Hilfe}$ der Programmsubkategorien erfolgt wiederum eine weitergehende Festlegung der übergeordneten Ziele auf der Kategorieebene. Sie bilden die erste Stufe, die eine Zieloperationalisierung erlaubt (z.B. Steigerung der Zahl der Abschlüsse an weiterbildenden Einrichtungen). Die auf der dritten Ebene rangierenden Programmelemente stellen dann ganz spezifische, identifizierbare Leistungen dar (z.B. Einrichtung von Förderkursen) und können, ähnlich wie bereits die Subkategorien im Hinblick auf die Programmkategorien, als Mittel zur Realisierung der Subkategorien betrachtet werden. Auf der vierten Ebene werden schlieblich als Programmsubelemente die sachlichen und personellen Ressourcen ausgewiesen ( $z . B$. Lehr- und Lernmitte 1). 1)

Die Analyse nationaler Ziele sowie die Programmstruktur des PPBS stellen demnach den Versuch dar, den Bereich öffentlicher Aufgaben mittels einer hierarchischen Ordnung systematisch zu erfassen. Ohne Zweifel gelingt es, die enorme Spannweite des Aufgabenbegriffes anschaulich zu dokumentieren. Damit wird nicht nur ein Beitrag zur Verbesserung der Transparenz geleistet, sondern auch Orientierungspunkte für grundsätzliche Möglichkeiten der Aufgabendimensionierung geliefert. Das PPBS trägt also zur Verdeutlichung des Problems der Mehrdimensionalität öffentlicher Aufgaben bei, lösen kann es hingegen dieses Problem nicht, denn das Planungsinstrument gibt keine Auskunft darüber, welche Ebene zweckmäßigerweise als Aufgabenebene gelten soll. Gleichwohl kann in diesem Zusammenhang auf einige Konsequenzen hingewiesen werden, die sich mit Blickrichtung auf das Erkenntnisinteresse dieser Untersuchung ergeben.

1) Vgl. E. Wille, Art. Offentlicher Haushalt IV: Finanz- und Aufgabenplanung, a.a.0., S. 608. Ausführlich dazu C. Böhret, Entscheidungshilfen für die Regierung, Opladen 1970, insbes. S. $182 \mathrm{ff}$. sowie H. Reinermann, Programmbudgets in Regierung und Verwaltung, Baden-Baden 1975, S. $120 \mathrm{ff}$. 
Allgemeine Ziele wie Freiheit, Gerechtigkeit oder Sicherheit haben zumindest auf den ersten Blick ausgesprochen integrativen Charakter, wird doch der Wunsch nach Realisierung dieser Ziele von kaum einem Mitglied der Gesellschaft ernsthaft in Frage geste11t. 1) Breiter Konsens scheint jedoch allein deshalb gewährleistet, weil allgemeine Zielformulierungen die Eigenschaft von Leerformeln aufweisen: Sie können jederzeit beliebig ausgefuillt werden und entziehen sich infolge mangelnder Operationalität jeglicher Kontrolle der Zielerreichung. GERHARD COLM, der Begründer der "National Goals Analysis", schlägt deshalb vor, die Zielbestimmung auf eine operationalisierbare Ebene zu verlagern und damit "konkrete Einzelziele" wie Landesverteidigung, Erziehung oder Gesundheit zur Zielformulierung heranzuziehen. ${ }^{2)}$ Allerdings hält COLM auch auf dieser Ebene eine weitgehende Ubereinstimmung über den grundsätzlichen Inhalt dieser Einzelziele für wahrscheinlich, was jedoch nicht heißt, daß über die Geschwindigkeit der Zielverwirklichung und dem geeigneten Mitteleinsatz sowie der relativen Priorität der Ziele nicht bereits beträchtliche Differenzen zwischen den Individuen der Gesellschaft bestehen können. 3)

Die Alternative, zur Bestimmung von Aufgaben möglichst eine untere und damit hinreichend operationalisierte Zielebene heranzuziehen, erweist sich allerdings aus mehreren Gründen ebenfalls als problematisch.

1) Als zumindest in der westlichen Welt ganz überwiegend anerkannte grundlegende Ziele werden etwa von Dahl und Lindblom Freiheit, Rationalität, Demokratie, subjektive Gleichheit, Sicherheit, Fortschritt und eine angemessene Berücksichtigung der individuellen und Gruppeninteressen genannt. Allerdings wird darauf hingewiesen, daß selbst diese Ziele als "instumental goals" im Hinblick auf die Verwirklichung absoluter Oberziele (prime goals) wie z.B. der Existenzsicherung des Menschen zu begreifen sind; $v g l$. R. A. Dahl und Ch. E. Lindblom, Politics, Economics, and Welfare, New York 1953, S. $28 \mathrm{ff}$.

2) Vgl. G. Colm, National Goals Analysis and Marginal Utility Economics, in: Finanzarchiv, N.F., Bd. 24 (1965), S. 218 f. Von den konkreten Einzelzielen (achievement goals) unterscheidet Colm die ordnungspolitischen Hauptaufgaben (performance goals), zu denen seiner Ansicht nach Vollbeschäftigung, angemessene Preisstabilität, Konsumentensouveränität und ein vernünftiger Ausgleich zwischen Arbeit und Freizeit gehören; vgl. dazu G. Colm, Art. Nationalbudget, in: HdSW, Bd. 7, Stuttgart u.a. 1961, S. 536.

3) $\mathrm{Vgl}$. G. Colm, National Goals Analysis and Marginal Utility Economics, a.a.0., S. 219 . 
Zunächst: Je konkreter eine Aufgabe inhaltlich umschrieben wird, desto enger wird notwendigerweise die Verknüpfung von Aufgabenbestimmung und Art und Weise der Aufgabenerfüllung. Offenbar wird dann häufig übersehen, daß die Durchführung einer Aufgabe grundsätzlich auf höchst unterschiedlichem Wege möglich ist. Symptomatisch hierfür ist das bei der Aufstellung von Haushaltsplänen übliche Vorgehen der Bedarfsanmeldungen von unten nach oben. Mit der Bereitstellung von sachlichen oder personellen Ressourcen werden hier i.d.R. gleichzeitig die mit ihrer Hilfe wahrzunehmenden Aufgaben fortgeschrieben und institutionell verankert, ohne nähere Prüfung, ob die praktizierte Wahrnehmungsform auch weiterhin als zweckmäßig zu betrachten oder u.U. die Aufgabe uberhaupt in Frage zu stellen ist. Diese weitverbreitete Identifizierung der Art und Weise der Aufgabenerfüllung mit der Aufgabenbestimmung versperrt den Ausblick auf neue, eventuell weit besser geeignete Formen der Aufgabenwahrnehmung, verhindert damit ein Denken in Alternativen und erschwert sowohl die Aufgabenanalyse als auch die Aufgabenkritik.

Aus dem ersten Punkt ergibt sich unmittelbar auch eine zweite Oberlegung. Hierarchisch geordnete Zielsysteme weisen Interdependenzen zwischen den einzelnen Strukturebenen auf, d.h. auf höherer Ebene gefällte Entscheidungen determinieren die Struktur der Ziele bzw. Aufgaben auf untergeordneter Ebene. So ist z.B. zur Erreichung des Zieles "saubere Umwelt" nicht nur eine Vielzahl alternativer Maßnahmen des öffentlichen Sektors denkbar, sondern grundsätzlich wäre ebenso vorstellbar, daß ein individueller und einklagbarer Rechtsanspruch auf eine saubere Umwelt allgemein anerkannt würde mit der Folge, daß die Wirtschaftssubjekte nun selbst ausreichende Vorkehrungen zur Vermeidung von Umweltverschmutzungen zu ergreifen hätten.

Schließich: Während zwischen den Zielebenen auftretende gegenseitige Abhängigkeiten als vertikale Interdependenzen bezeichnet werden können, existieren daruber hinaus horizontale Interdependenzen infolge der multiplen Zielsetzung vieler Aufgaben, d.h. ein und dieselbe Aufgabe kann diverse Funktionen erfillien. So geht mit der Bereitstellung einer öffentlichen Parkanlage 
nicht nur eine Verbesserung des Freizeitangebots für die Bevölkerung einher, sondern gleichzeitig dient die Parkanlage der Reinhaltung der Luft. Wird zur Aufgabenbestimmung eine untere Ebene innerhalb der Zielstruktur herangezogen, so werden auch diese Interdependenzen zwangsläufig vernachlässigt.

Anhand der Obersicht 2 lassen sich aus den Erwägungen einige SchluBfolgerungen ziehen. Als Grundlage für die weitere Unter-

Obersicht 2: Strukturebenen und Dimensionen öffentlicher Aufgaben

\begin{tabular}{|c|c|c|c|}
\hline STRUKTUREBENE & $\begin{array}{l}\text { EIGENSCHAFT DER } \\
\text { STRUKTUREBENE }\end{array}$ & DIMENSION & $\begin{array}{l}\text { Beispiel: } \\
\text { BILDUNGSWESEN }\end{array}$ \\
\hline Ziele & \multirow{2}{*}{$\begin{array}{l}\text { keine Operationa- } \\
\text { lisierung } \\
\text { möglich }\end{array}$} & $\begin{array}{l}\text { allgeme ine } \\
\text { nationale } \\
\text { Ziele }\end{array}$ & $\begin{array}{l}\text { Erhöhung des } \\
\text { Bildungsniveaus } \\
\text { der Bevölkerung }\end{array}$ \\
\hline Leitbilder & & $\begin{array}{l}\text { allgemeine } \\
\text { Charakteri- } \\
\text { sierung na- } \\
\text { tionaler } \\
\text { Ziele }\end{array}$ & $\begin{array}{l}\text { Ausbau einer } \\
\text { chancengerech- } \\
\text { ten und lei- } \\
\text { stungsfähigen } \\
\text { schulischen } \\
\text { Bildung }\end{array}$ \\
\hline Funktionen & $\begin{array}{l}\text { erste Stufe der } \\
\text { Zieloperationa- } \\
\text { lisierung }\end{array}$ & $\begin{array}{c}\text { öffentliche } \\
\text { Aufgaben } \\
\text { (i.e.S.) }\end{array}$ & $\begin{array}{l}\text { Steigerung der } \\
\text { Zahl der Ab- } \\
\text { schlüsse an } \\
\text { weiterbildenden } \\
\text { Einrichtungen }\end{array}$ \\
\hline Produkte & $\begin{array}{l}\text { operationaler } \\
\text { Output ohne di- } \\
\text { rekten Zielbezug }\end{array}$ & $\begin{array}{l}\text { öffentliche } \\
\text { Leistungen, } \\
\text { öffentliche } \\
\text { Guiter }\end{array}$ & $\begin{array}{l}\text { differenziertes } \\
\text { Bildungsangebot; } \\
\text { Einrichtung von } \\
\text { Förderkursen }\end{array}$ \\
\hline \multirow{2}{*}{ Ressourcen } & $\begin{array}{l}\text { sachlicher und } \\
\text { personeller } \\
\text { Input }\end{array}$ & $\begin{array}{l}\text { private und } \\
\text { offentliche } \\
\text { Produktions- } \\
\text { mittel }\end{array}$ & $\begin{array}{l}\text { Schulen, Lehr- } \\
\text { mittel, Lehrer }\end{array}$ \\
\hline & $\begin{array}{l}\text { monetärer } \\
\text { Input }\end{array}$ & $\begin{array}{l}\text { öffentliche } \\
\text { Ausgaben }\end{array}$ & $\begin{array}{l}\text { Ausgaben für } \\
\text { Schulen, Lehr- } \\
\text { mittel, Lehrer }\end{array}$ \\
\hline
\end{tabular}


suchung scheint es weder sinnvoll, eine obere Zielebene zu wählen, da diese eine Operationalisierung der Aufgabenstellung nicht zuläßt, noch scheint es zweckmäßig, die weitere Analyse auf einer unteren Ebene anzusetzen, da in diesem Fall insbesondere durch den Konnex von Aufgabenbestimmung und Aufgabendurchführung der Mittelcharakter sowohl der getätigten Ausgaben und eingesetzten Produktionsmittel (Inputs) als auch der erbrachten Leistungen (Outputs) verschleiert wird. Dem Erkenntnisinteresse dieser Arbeit entspricht es dagegen wesentlich eher, den Aufgabenbegriff auf der Funktionenebene anzusiedeln, zeichnet sich doch hier die Aufgabenbestimmung durch einen direkten zielbezug aus und wird gleichzeitig die erste Stufe der Zielstruktur genutzt, die eine Operationalisierung erlaubt. Die Funktionenebene besitzt damit die Vorteile oberer und unterer Zielebenen, ohne jedoch auch deren Nachteile aufzuweisen.

\section{Methodische Ansätze einer Abgrenzung öffentlicher von privaten Aufgaben}

Die skizzenhafte Wiedergabe einiger Aussagen wissenschafti icher Disziplinen hat u.a. gezeigt, daß die Diskussion uber eine Abgrenzung der Aufgabenbereiche auf Grundlage ganz verschiedener Ansätze erfolgen kann. Nach dem methodischen Vorgehen unterscheiden sich die Ansätze derart, daß eine Gruppe versucht, öffentliche Aufgaben zu beschreiben, eine zweite Gruppe danach strebt, die Erklärungsfaktoren des gewachsenen und gegenwärtig vorhandenen Aufgabenbestandes zu liefern und schließlich eine dritte Gruppe erörtert, welche Aufgabenverteilung in der Volkswirtschaft als wïnschenswert anzusehen ist. Damit lassen sich die im Grundsatz unterscheidbaren Ansätze auf folgende kurze Fragestellungen verengen: "Was ist?", "Warum ist es so?" und "Wie sollte es sein?".

Oder etwas exakter auf unsere Fragestellung projiziert:

- Mit Hilfe welcher Kriterien können die gegenwärtig in der Bundesrepublik Deutschland als öffentliche Aufgaben betrachteten Aktivitäten von den privaten Aufgaben abgegrenzt werden (deskriptiver Ansatz)? 
- Welche Bestimmungsgründe können zur Erklärung des gegenwärtigen Bestandes und der Entwicklung öffentlicher Aufgaben herangezogen werden (positiver Ansatz)?

- Welche Kriterien sind für die Ableitung einer ökonomisch zweckmäßigen Aufgabenverteilung zwischen öffentlichem und privatem Sektor maßgebend (normativer Ansatz)?

Innerhalb der Wirtschaftswissenschaft ist heute weitgehend die methodologische Trennung zwischen positiver und normativer Betrachtungsweise gebräuchlich. 1) Ganz allgemein gilt, daß die positive Theorie Ist-Aussagen aufstellt, mit deren Hilfe UrsacheWirkungs-Zusammenhänge erklärt sowie Prognosen erstellt werden können. Das Ziel der normativen Theorie besteht dagegen darin, aus einem Kranz vorgegebener Prämissen Soll-Aussagen für bestimmte Problembereiche abzuleiten. In der Literatur findet sich hingegen selten eine Differenzierung zwischen rein deskriptiver Betrachtung und den darüber hinausgehenden Erklärungsansätzen. 2) Die Dreiteilung der Betrachtungsweise erscheint zweckmäßig, da Mißverständnisse und Irrtümer in der wissenschaftlichen Auseinandersetzung zu einem beträchtlichen Teil auf die Nichtbeachtung unterschiedlicher Diskussionsansätze zurückzuführen sind. Gleichwohl kann diese Trennung lediglich konzeptionellen Charakter besitzen, deren Vorteil sich in einer möglichst klaren Darstellungsweise der drei grundsätzlichen Möglichkeiten der Behandlung öffentlicher Aufgaben erschöpft.

1) Die Unterscheidung von "positive science" und "normative science" wurde bereits 1891 erstmals von John Neville Keynes getroffen; vgl. J. N. Keynes, The Scope and Method of Political Economy, 4. Auf1., London, New York 1955, S. $9 \mathrm{ff}$.

2) So z.B. P. Streeten, Programs and Prognoses, QJE, Bd. 68 (1954), S. 355 ff.; 0 . Morgenstern, Deskriptive, Predictive, and Normative Theory, in: Kyklos, Bd. 25 (1972), S. $699 \mathrm{ff}$.; H. C. Recktenwald, Staatsausgaben in säkularer Sicht, in: Theorie und Praxis des finanzpolitischen Interventionismus. F. Neumark zum 75. Geburtstag, hrsg. von H. Haller u.a., Tübingen 1970, S. 407. Differenzierter dazu K. Schmidt, Zu einigen Theorien über die relative Ausdehnung der öffentlichen Ausgaben, in: Finanzarchiv, N.F., Bd. 24 (1965), S. 193. 


\section{Deskriptiver Ansatz}

Die Deskription liefert Informationen darüber, was gegenwärtig in der Bundesrepublik Deutschland und damit zeit- und raumbezogen als öffentliche Aufgabe angesehen wird. Darüber hinaus sind mit ihrer Hilfe Längsschnitts- und Querschnittsvergleiche möglich. ${ }^{1)}$ So kann beispielsweise gezeigt werden, daß eine Aufgabe, die heute von öffentlichen Institutionen wahrgenommen wird, vor einigen Jahrzehnten noch durch Private erfült wurde und umgekehrt oder daB eine Aufgabe, die in der Bundesrepublik $z u$ den öffentlichen Aufgaben zählt, z.B. in den USA dem privaten Bereich zugeordnet wird. Eine bloBe Beschreibung leistet dagegen keinen Beitrag zur Beantwortung der Frage, warum eine Aufgabe als öffentlich bzw. privat betrachtet wird, und liefert auch keine Aussage darüber, inwieweit eine bestimmte Aufgabenverteilung ökonomisch zweckmäßig ist.

Die bereits dargelegte Komplexität öffentlicher Aufgaben läBt vermuten, daß Versuche, öffentliche und private Aufgaben mittels eines einzigen umfassenden Kriteriums abgrenzen zu wollen, von vornherein zum Scheitern verurteilt sind. Außerdem wäre hierzu eine präzise Begrifflichkeit erforderlich, an der es aber gerade im Hinblick auf die Beschreibung öffentlicher Aufgaben mangelt. Deshalb soll der Versuch unternommen werden, im Rahmen einer systematisch angelegten Deskription die Tauglichkeit verschiedener, unterschiedlichen Fragestellungen Rechnung tragender Kriterien für eine Aufgabenabgrenzung zu überprüfen. Einzelne Kriterien können dabei zu Kriterienkategorien zusammengefaßt werden, und zwar zu

- funktionalen Kriterien, welche die Zuordnung anhand der speziell öffentlichen oder privaten Zwecksetzung einer Aufgabe vornehmen;

1) Das bedeutet, daß auch sämtliche statistischen Erhebungen dem deskriptiven Ansatz zugeordnet werden können. 
- institutionellen Kriterien, welche die "offentlichkeit oder "Privatheit" einer Aufgabe allein von der Zugehörigkeit des Aufgabenträgers zum öffentlichen bzw. privaten Sektor abhängig machen;

- technisch-ökonomischen Kriterien, die entweder aus ganz spezifischen Eigenschaften einer Aufgabe auf ihre "offentlichkeit" schließen oder aber die Ausgestaltung des Koordinationsmechanismus, uber den die Erfüllung der Aufgabe erfolgt, zum entscheidenden Merkmal erheben.

Um die Tauglichkeit der Kriterien für eine Aufgabenabgrenzung prüfen zu können, müssen wiederum konkrete Anforderungen an die Kriterien selbst gestellt werden:

1. sollten sie in Bezug auf die jeweilige Fragestellung präzise und eindeutig unterscheidbare Ausprägungen aufweisen, insbesondere sollten uberschneidungen vermieden werden (Eindeutigkeit), und

2. sollten die abzugrenzenden Aufgaben ohne Rest diesen unterscheidbaren Ausprägungen ebenso eindeutig zugeordnet werden können (Zuordnungsmöglichkeit).

\section{Positiver Ansatz}

Anknüpfungspunkt der positiven Theorien ${ }^{1)}$ sind die tatsächlich $z u$ beobachtenden privaten und öffentlichen Aktivitäten. Von vornherein orientieren sie sich nicht an einem Soll-Zustand, sondern ihr Anliegen ist die Erklärung ${ }^{2)}$ realer Verhältnisse. 3 ).

1) Häuser spricht in diesem Zusammenhang von pragmatischen Theorien; vgl. K. Häuser, Uber Ansätze zur Theorie der Staatsausgaben, in: Beiträge und Theorie der öffentlichen Ausgaben, hrsg. von H. Timm und H. Haller, SchdVSocpol, N.F., Bd. 47, Berlin, S. 57.

2) Die Bedeutung der Erklärung innerhalb der Theorienbildung wird besonders von Buchanan hervorgehoben, wenn er schreibt: "Explanation is the primary function of theory". J. M. Buchanan, The Demand and Supply of Public Goods, 2. Auf1., Chicago 1969, S. 1. Neben der Erklärung können aber auch die Prognose der zukünftigen Entwicklung sowie die Erarbeitung von Grundlagen für wirtschaftspolitische Empfehlungen als Funktionen der positiven Analyse genannt werden; vgl. dazu E. R. Morss, Some Thoughts on the Determinants of State and Local Expenditures, NTJ, Bd. 19 (1966), S. 96.

3) Vgl. K. Littmann, Problemstellung und Methoden der heutigen Finanzwissenschaft, HdF, Bd. 1, 3. Aufl., Tübingen 1977, S. 103. 
Im Mittelpunkt des Interesses stehen demnach die Bestimmungsfaktoren, die eine historisch-charakteristische Struktur von privaten und öffentlichen Aufgaben in einem bestimmten Lande hervorgebracht haben. So kann die positive Analyse darüber Auskunft geben, warum bestimmte Güter in der Vergangenheit über den Markt angeboten wurden, heute hingegen öffentlich bereitgestellt werden und umgekehrt. Sie kann daneben Begründungen dafür liefern, weshalb einige Tätigkeiten, die in der Bundesrepublik Deutschland als öffentliche Aufgaben geführt werden, gleichzeitig im Ausland zum Bereich der öffentlichen Aufgaben gehören und vice versa. Solche Erklärungsansätze können somit u.a. aufschlußreiche Erkenntnisse über Ziele und Aktivitäten politischer Handlungsträger - beispielsweise rationales und fehlerhaftes Verhalten von Politikern, Einflußnahme von Verwaltungen und Verbänden, rekurrenter Anschluß einmal gefällter Entscheidungen - vermitteln.1)

Obschon bestimmte Aspekte der positiven Analyse später an verschiedenen Stellen wieder aufzunehmen sind, wird in dieser Arbeit darauf verzichtet, neben der Erörterung der deskriptiven und normativen Kriterien für eine Aufgabenabgrenzung und -verteilung die möglichen Bestimmungsgründe der empirisch feststellbaren Struktur privater und öffentlicher Aktivitäten gesondert zu untersuchen. Zwar mangelt es bisher an einer umfassenden positiven Analyse der Aufgabenverteilung ${ }^{2}$, es ist aber nicht zu erwarten, daß eine geschlossene Theorie aus ökonomischer sicht überhaupt entwickelt werden kann. Die gegenwärtige Aufgabenverteilung ist nun einmal nicht allein das Resultat ökonomischer

1) Vgl. G. Krause-Junk, Abriß der Theorie von den öffentlichen Gütern, HdF, Bd. 1, 3. AufT., Tübingen 1977, S. 690.

2) Dagegen existiert eine Reihe von Beiträgen zur positiven Theorie der Staatsausgaben; im überblick vgl. dazu F. L. Pryor, Elements of a Positive Theory of Public Expenditures, in: Finanzarchiv. N.F., Bd. 26 (1967), S. $405 \mathrm{ff}$. sowie G. Schwarting, Positive Theorien der Staatsausgaben: Ein kritischer Uberblick, SZVS 116. Jg. (1980), S. $67 \mathrm{ff}$. Unterstellt wird in diesen Ansätzen, die staatlichen Ausgaben seien ein hinreichend genauer Indikator für die Aktivität des Staates. Zu den Problemen dieser Annahme vgl. zweiter Teil, Abschnitt II, Punkt 4.3. dieser Arbeit. 
Zweckmäßigkeitserwägungen. Im Gegenteil, häufig werden politische Entscheidungen getroffen, die nur wenig durch ökonomische Rationalität geprägt sind. Die Wirtschaftswissenschaft kann deshalb zur Erklärung der beobachtbaren Aufgabenverteilung in der Volkswirtschaft nur einen vergleichsweise bescheidenen Beitrag leisten. Geschichts- und Politikwissenschaftler düften eher berufen sein, einen positiven Ansatz zur Erklärung der Aufgabenverteilung auszufiullen. Die Wirtschaftswissenschaft ist jedoch gerade aus diesem Grunde umso mehr gefordert, dem Politiker Informationen daruber zu liefern, welche Aufgabenverteilung unter ökonomischen Gesichtspunkten als zweckmäßig zu betrachten ist.

\section{Normativer Ansatz}

Normative Aussagen beruhen zwangsläufig auf Wertvorstellungen, die auch dann, wenn sie vom Wissenschaftler explizit gemacht werden, den Charakter eines subjektiven Ansatzes beibehalten. ${ }^{1}$ ) Normen sind eben nicht aus beobachtbaren Fakten ableitbar, sondern normative Aussagen ergeben sich aus anderen, allgemeineren Soll-Sätzen. Deshalb braucht auch "die Welt des Sollens... wenig oder gar nichts mit der Welt des Seins gemeinsam zu haben". 2)

Die Grundfrage des normativen Ansatzes lautet demnach: Welche Struktur hat eine ökonomisch wïnschenswerte Aufgabenverteilung zwischen privatem und öffentlichem Sektor? Die normative Analyse wird dabei in der wirtschaftswissenschaftlichen Literatur

1) Inwieweit normative Aussagen uiberhaupt in den Bereich der Wissenschaft gehören, soll hier nicht weiter erörtert werden. Es mag genügen, darauf zu verweisen, daß insbesondere in der Finanzwissenschaft die Bedeutung normativer Aussagen unbestritten ist. Beispielhaft sind nur die Fundamentalprinzipien der Besteuerung, die Haushaltsgrundsätze und die Ableitung eines optimalen Budgetvolumens zu nennen. Statt vieler vgl. hierzu die 1962 auf der Arbeitstagung des Vereins für Socialpolitik gehaltenen Vorträge von Weisser und Albert; G. Weisser, Das Problem der systematischen Verknüpfung von Normen und von Aussagen der positiven Okonomik in grundsätzlicher Betrachtung, erläutert anhand des Programms einer sozialwissenschaftlichen Grunddiszipl in aus Empfehlungen und Warnungen ( $S$. $16 \mathrm{ff}$. ) und $H$. Albert, Wertfreiheit als methodisches Prinzip, in: Probleme der normativen Okonomik und der wirtschaftlichen Beratung, hrsg. von $E$. von Beckerath und H. Giersch, SchdVSocpol, N.F., Bd. 29, Berlin 1963, S. $32 \mathrm{ff}$.

2) G. Krause-Junk, Abriß der Theorie von den öffentlichen Gütern, a.a.0., S. 691; vgl. auch 0. Morgenstern, a.a.0., S. $710 \mathrm{f}$. 
in aller Regel mit der Wohlfahrtstheorie (welfare economics) gleichgesetzt, die unter Verwendung einer gesellschaftlichen Wohlfahrtsfunktion das Ziel der Wohlstandsmaximierung formuliert und unter bestimmten Bedingungen ökonomische Bestlösungen abzuleiten versucht. Die methodologischen Schwierigkeiten der normativen Analyse bestehen allerdings vornehmlich darin, $d a B$ a priori festgelegt werden muß, unter welchen Bedingungen ein Optimum erreicht ist, genauer, wodurch sich eine - in diesem Fall ökonomisch-soziale - Bestlösung der Aufgabenzuordnung auszeichnen soll. 
Zweiter Teil

\section{DESKRIPTIVE KRITERIEN EINER ABGRENZUNG ÖFFENTLICHER VON PRIVATEN AUFGABEN}

\section{Funktionale Abgrenzungskriterien}

Soll eine deskriptive Aufgabenabgrenzung allein nach funktionalen Gesichtspunkten vorgenommen werden, so ist der mit einer konkreten Tätigkeit verbundene öffentliche oder private Zweck ausschlaggebend für die Identifikation einer Aktivität als öffentliche oder private Aufgabe. In der Literatur finden sich zahlreiche Versuche einer funktionalen Beschreibung öffentlicher Aufgaben, die grob drei Richtungen zugeordnet werden können. Die erste Richtung geht davon aus, daß es einen festen Grundbestand öffentlicher Funktionen gibt, die einen sog. "öffentlichen Kernbereich" bilden. Die zweite Richtung unternimmt den Versuch, öffentliche Aufgaben zu systematisieren, indem der vorhandene Aufgabenbestand nach bestimmten formalen Gesichtspunkten oder mit Hilfe materieller Kriterien gegliedert wird. 1 ) Die dritte Richtung schlieBlich versucht aus einer Gegenüberstellung von "Gemeinwohl" und "Eigennutzstreben" inhaltliche Kriterien für eine Aufgabenabgrenzung zu gewinnen.

1. Bestimmung eines "öffentlichen Kernbereichs"

In der Literatur besteht weitgehender Konsens daruber, da $B$ es einen "Kernbereich des offentlichen" gibt, d.h. einen allgemein anerkannten Grundbestand von Aufgaben, deren öffentliche Funktionen so klar hervortreten, daB eine eindeutige Identifikation

1) Oberblickartig dazu G. F. Schuppert, Die öffentliche Aufgabe als Schlüsselbegriff der VerwaTtungswissenschaft, in: Verwaltungsarchiv, Bd. 71 (1980), S. 309.; R. Hillmann und H. von Wersebe, a.a.0., S. $71 \mathrm{ff}$.; H. P. Bull, a.a.0., S. $213 \mathrm{ff}$. 
dieser Aufgaben als öffentliche vorgenommen werden kann und gleichzeitig die Notwendigkeit ihrer Bereitstellung durch den statichen Sektor als unumstritten angesehen wird.

Strittig bleibt in diesen Fällen jedoch gleich dreierlei.

Erstens mangelt es bereits an einem einheitlichen Begriff für diesen Komplex öffentlicher Aufgaben. Sie werden bezeichnet als "originäre"1), "wesentliche"2) oder "staatsnotwendige"3) Aufgaben, als "Staatsaufgaben aus der Natur der Sache"4), als "geborene" 5 ) Aufgaben oder als "sine qua non activities"6), um nur einige Termini zu nennen. 7 )

Zweitens besteht auch bezüglich des Umfangs des öffentlichen Kernbereichs und damit hinsichtlich der Beantwortung der Frage, welche Aufgaben denn diesem Kernbereich zuzuordnen sind, keineswegs Ubereinstimmung. Durchgehend wird zwar die Aufrechterhaltung der äußeren und inneren Sicherheit genannt, daneben werden von einigen Autoren aber auch beispielsweise die Ausstattung des Staates mit finanziellen Mitteln ${ }^{8}$ ), bau- und gesundheitsbehördliche Aufgaben ${ }^{9}$ ) oder die Sicherung des Eigentums ${ }^{10}$ ) ausdrücklich

1) H.P.Bul1, a.a.0., S. 99.

2) W. Leisner, Werbefernsehen und öffentliches Recht, Berlin 1967, S. $14 \mathrm{ff}$.

3) P. Kirchhof, Der Begriff der hoheitsrechtlichen Befugnisse in Artikel 33 Absatz IV des Grundgesetzes, Diss., Muinchen 1968, S. 111.

4) M. Krautzberger, a.a.0., S. 53.

5) K. Schmidt, Zur Geschichte der Lehre von den Kollektivbeduirfnissen, in: Systeme und Methoden in den Wirtschafts- und Sozialwissenschaften, E. von Beckerath zum 75. Geburtstag, hrsg. von W. Kloten u.a., Tübingen 1964, S. 361 sowie G. Baum, Probleme der Privatisierung öffentlicher Aufgaben, in: Privatisierung - Gewinn für wen?, Vorträge und Diskussionsbeiträge der 21. beamtenpolitischen Arbeitstagung des Deutschen Beamtenbundes, Bonn 1980 , S. 26.

6) R. Rose, On the Priorities of Government: A Developmental Analysis of PubTic PoTicies, EJPR 4 (1976), S. $249 \mathrm{f}$.

7) Ausführlicher vgl. dazu H.P.Bull, a.a.0., S. 99 ff. und M. Krautzberger, a.a.0., S. $49 \mathrm{ff}$.

8) So R. Rose, a.a.0., S. 250.

9) Vgl. G. Baum, a.a.0., S. 26.

10) $\mathrm{Vgl.K}$. Schmidt, Zur Geschichte der Lehre von den Kollektivbedürfnissen, a.a.0., S. 361. 
als dem öffentlichen Kernbereich zugehörige Aufgaben angesehen. 1)

Drittens wird dieser "gesicherte Bestand öffentlicher Aufgaben" sehr unterschiedlich begrüdet. Während LEISNER den zwingenden Einsatz von Hoheitsgewalt zum konstitutiven Kriterium erhebt ${ }^{2}$, ist für KIRCHHOF entscheidend, daß im Hinblick auf diese Aufgaben eine Intervention des States als unentbehrlich und unersetzbar angesehen wird. ${ }^{3)}$ Noch weiter geht ROSE, der den von ihm abgeleiteten Kernbereich öffentlicher Aufgaben damit begründet, daß ohne diesen der moderne Staat gar nicht denkbar wäre. Es handele sich deshalb auch um begriffsnotwendige Aktivitäten (defining activities). ${ }^{4}$ ) Keiner dieser Begründungsversuche vermag indes zu überzeugen. BULL kritisiert, daß es eben nicht eindeutig feststellbar ist, in welchen Fällen der Einsatz von Hoheitsgewalt zwingend erforderlich ist, ${ }^{5}$ ) und den Hinweis auf unentbehrliche und unersetzbare Aktivitäten des Staates hält KRAUTZBERGER für zu unverbindlich und darüber hinaus zeitlichen Veränderungen unterworfen. ${ }^{6)}$ In der Tat scheint es zweifelhaft, ob langfristig überhaupt ein Kernbereich öffentlicher Aufgaben feststellbar ist. Allein der Hinweis, daß es zu (fast) jeder staatlichen Tätigkeit irgendwann einmal eine privatwirtschaftliche Alternative gab, ${ }^{7)}$ läßt hier einige skepsis angebracht erscheinen.

1) Weitere in der Literatur erwähnte Bereiche nennt M. Krautzberger, a.a.0. S. 50 .

2) Vgl. W. Leisner, Werbefernsehen und öffentliches Recht, a.a.0., S. 17.

3) $\mathrm{Vgl}$. P. Kirchhof, a.a.0., S. $111 \mathrm{ff}$.

4) Vgl. R. Rose, a.a.0., S. $249 \mathrm{f}$.

5) Vgl. H.P.Bull, a.a.0., S. 100.

6) Vgl. M. Krautzberger, a.a.0., S. 52.

7) So hat z.B. Littmann dargelegt, daß es "- abgesehen von der Rechtsprechung keine Tätigkeit des Staates gibt, die historisch nicht auch einmal von Privaten ausgeübt worden wäre." K. Littmann, Zunehmende Staatstätigkeit und wirtschaftiche Entwicklung, Köln und Opladen 1957, S. 35. 
Schlieblich: Selbst wenn es problemlos gelänge, in einem ersten Schritt eine Anzahl Aufgaben zu benennen, die eine Art öffentlichen Kernbereich bilden, so ist damit nur recht wenig gewonnen, sind doch bei dem Anliegen, umfassend und systematisch öffentliche und private Aufgaben abzugrenzen, vor allem die über die unstrittigen Fälle hinausgehenden Aufgabenbereiche von Interesse.

2. Systematisierungsversuche öffentlicher Aufgaben

2.1. Aufgabengliederungen nach formalen Kriterien

$\S 11$ des Gesetzes über die Grundsätze des Haushaltsrechts des Bundes und der Länder (Haushaltsgrundsätzegesetz) sowie $\S 14$ der Bundeshaushaltsordnung bestimmen, daB den Haushaltsplänen als Anlage eine Funktionenübersicht beizufuigen ist. Unabhängig von der institutionellen - d.h. dem Ministerialprinzip folgenden - Gruppierung des Haushaltsplanes werden mit Hilfe der Funktionenubersicht alle Einnahmen und Ausgaben nach bestimmten Aufgabengebieten bzw. Funktionen gegliedert. Solche Funktionen sind z.B. Bildungswesen, soziale Sicherung sowie Gesundheit, Sport und Erholung. Zweck der Funktionenübersicht ist es aufzuzeigen, in welcher Höhe finanzielle Mittel für konkrete Aufgaben verausgabt werden, sie gibt mithin Auskunft über das AusmaB der mit der Erfüllung bestimmter vom Staat wahrgenommener Aufgaben einhergehenden Ausgabentätigkeit des Staates. Wenn deshalb der Haushaltsplan gelegentlich als "Regierungserklärung in Zahlen" oder exakter noch als "monetärer Ausdruck des politischen Handlungsprogramms der Regierung"1) bezeichnet wird, so gilt dies allenfalls für die dem Haushaltsplan als Anlage beigefügte Funktionenübersicht. Das Gliederungsschema für diese Funktionenübersicht wird dem sog. Funktionsplan entnommen (vgl. Obersicht 3 ), in dem die vom Staat gegenwärtig wahrgenommenen (ausgabenwirksamen Aufgaben nach Hauptfunktionen, Oberfunktionen und Funk-

1) K.-H. Hansmeyer und B. Rürup, Staatswirtschaftliche Planungsinstrumente, 2. AufT., Tubingen, DússeTdorf 1975, S. 8. 
Obersicht 3: Haushaltssystematik des Bundes - Funktionenplan

\section{ALLGEMEINE DIENSTE}

01 Politische Führung und zentrale Verwaltung

02 Auswärtige Angelegenheiten

03 Verteidigung und Zivilschutz

04 Offentliche Sicherheit und Ordnung

05 Rechtsschutz

1 BILDUNGSWESEN, WISSENSCHAFT, FORSCHUNG, KULTURELLE ANGELEGENHEITEN

11 Verwaltung

12 Schulen

13 Wissenschaftliche Hochschulen

14 Sonstige Hochschulen

15 Sonstiges Bildungswesen

16/17 Forschung außerhalb der Hochschulen (ohne Forschung der Verteidigung 036)

18 Kunst- und Kulturpflege

19 Kirchliche Angelegenheiten

2 SOZIALE SICHERUNG, SOZIALE KRIEGSFOLGEAUFGABEN, WIEDERGUTMACHUNG

21 Verwaltung

22 Sozialversicherung einschließlich Arbeits losenversicherung

23 Familien-, Sozial- und Jugendhilfe

24 Soziale Leistungen für Folgen von Krieg und politischen Ereignissen

25 Arbeitsmarktpolitik und Arbeitsschutz

26 Naturkatastrophen

27 Sonstige soziale Angelegenheiten

3 GESUNDHEIT, SPORT UND ERHOLUNG

31 Einrichtungen und Maßnahmen des Gesundheitswesens

32 Sport und Erholung

33 Reinhaltung von Luft, Wasser und Erde

4 WOHNUNGSWESEN, RAUMORDNUNG UND KOMMUNALE GEMEINSCHAFTSDIENSTE

41 Wohnungswesen

42 Raumordnung, Landesplanung

43 Kommunale Gemeinschaftsdienste

5 ERNÄHRUNG, LANDWIRTSCHAFT UND FORSTEN

51 Verwaltung (ohne Betriebsverwaltung)

52 Produktivitäts- und absatzfördernde Maßnahmen

53 Einkommensstabilisierende Maßnahmen

54 Sonstige Förderungsmaßnahmen
6 ENERGIE- UND WASSERWIRTSCHAFT, GEWERBE, DIENSTLEISTUNGEN

61 Verwaltung

62 Energie- und Wasserwirtschaft, Kulturbau

63 Bergbau und verarbeitendes Gewerbe

64 Handel

65 Förderung des Fremdenverkehrs

$66 \mathrm{Geld}$ - und Versicherungswesen

67 Sonstige Dienstleistungen

68 Sonstige Bereiche

69 Regionale Wirtschaftsförderung

7 VERKEHRS- UND NACHRICHTENWESEN

71 Verwaltung

72 Straßen

73 Wasserstraßen und Häfen

74 Schienenverkehr

75 Luftfahrt

76 Wetterdienst

77 Nachrichtenwesen

78 Sonstige Bereiche

8 WIRTSCHAFTSUNTERNEHMEN, ALLGEMEINE GRUND-, KAPITAL - UND SONDERVERMOGEN

81 Land- und forstwirtschaft 1 iche Unternehmen

82 Versorgungsunternehmen

83 Verkehrsunternehmen

84 Kombinierte Versorgungs - und Verkehrsunternehmen

85 Bergbau - und Gewerbeunternehmen

86 Sonstige Wirtschaftsunternehmen

87 Allgeme ines Grund-, Kapitalund Sondervermögen

\section{ALLGEMEINE FINANZWIRTSCHAFT}

91 Steuern und allgemeine Finanzzuwe isungen

92 Schulden

93 Versorgung

94 Rücklagen

95 Sonstiges

97 Abwickiung der Vorjahre

99 Haushaltstechnische Verrechnungen 
tionen geordnet sind. 1) Dem für Bund und Länder einheitlichen Funktionenplan ${ }^{2}$ ) wird daher auch die Eigenschaft eines Staatsaufgabenkataloges zugeschrieben.

Ein solcher Aufgabenkatalog leistet allerdings für die Abgrenzung öffentlicher von privaten Aufgaben vornehmlich aus zwei Gründen recht wenig:

Erstens stellt der Funktionenplan lediglich eine Bestandsaufnahme staatlich wahrgenommener Aufgaben dar, d.h. ihm kommt eher der Charakter einer bloßen Enumeration staticher Funktionen zu. Das Gliederungsschema folgt dabei weder einem durchgehend einheitlichen Prinzip ${ }^{3)}$ noch liefert es Unterscheidungsmerkmale für öffentliche und private Aufgaben. Deskriptive Kriterien für eine Aufgabenabgrenzung können dem Funktionenplan daher nicht entnommen werden.

Zweitens geben die Funktionen- bzw. Gliederungspläne lediglich darüber Auskunft, mit welchen Aufgaben sich die verschiedenen Organisationseinheiten Bund, Länder und Gemeinden "befassen". Die Funktionenübersicht und die Einzelpläne spiegeln darüber hinaus das finanzielle Engagement dieser Aufgabenbefassung der jeweiligen Organisationseinheiten wider. Fragen einer funktio-

1) Vgl. Bundesministerium der Finanzen (Hrsg.), Verwaltungsvorschriften zur Haushaltssystematik des Bundes, Funktionenplan.

2) Das Pendant zum staatlichen Funktionenplan bildet der Gliederunasplan des Kommunalhaushalts. Beide unterscheiden sich nur geringfügig. Ein grundsätzlicher Unterschied zwischen staatlichem und kommunalem Haushaltsplan besteht hingegen darin, daß funktionale Gesichtspunkte im Gemeindehaushalt nicht lediglich als Anlage Beruicksichtigung finden, sondern infolge der nach dem Gliederungsplan vorgenommenen Klassifizierung der Einnahmen und Ausgaben der Einzelpläne des Verwaltungs- und Vermögenshaushalts als wesentlicher Bestandteil in den kommunalen Haushaltsplan eingehen.

3) Zu Recht kritisiert Brinkmann, daß aufgrund der mangelhaften Berücksichtigung seiner Mehrdimensionalität der Funktionenplan nicht eindeutig sei. Dies gilt zum einen deshalb, weil identische Funktionen an verschiedenen Stellen des Planes mit unterschiedlichen Kennziffern vorkommen, zum anderen, weil Aufgaben außer mit der ihr zugewiesenen Funktion ebenfalls noch mit alternativen Kategorien (Titel) des Planes gekennzeichnet werden können. Brinkmann kommt deshaib zu dem Urteil, daß alle Aussagen, die auf den Funktionenplan in seiner heutigen Form beruhen, mehr oder weniger sinnlos sind; vgl. G. Brinkmann, Der Funktionenplan. Anmerkungen eines Verwunderten, in: Der öffentliche Haushalt, Jg. 13 (1972), S. $77 \mathrm{ff}$. , insbesondere S. 83. 
nalen Aufgabenabgrenzung können damit allerdings zwangsläufig nur unzureichend beantwortet werden. Indem sich die Pläne nämlich auf die Darstellung des Betätigungsfeldes jeweils einer staatlichen Organisationseinheit beschränken, werden in vielen Fällen zum einen nur Teilfunktionen eines funktional zusammengehörenden Aufgabenkomplexes erfaßt, d.h. sie bilden nur einen Ausschnitt der Gesamtaufgabe $a b$, und zum anderen geben sie keine Auskunft über die Art und Weise der Aufgabenbefassung, d.h. über die organisatorische Ausgestaltung der Aufgabenerfüllung (Gesetzgebung, Finanzierung, Durchführung etc.).

In den Funktionen- und Gliederungsplänen werden somit horizontale und vertikale Aufgabenverflechtungen weitgehend vernachläs sigt. Unbeantwortet bleibt damit auch die für eine Aufgabenabgrenzung bedeutsame Frage, ob staatliche organisationseinheiten z.B. lediglich die Verwaltungskosten für die Kontrolle von Auflagen tragen oder finanzielle Förderungen vornehmen, im übrigen aber die Durchführung einer Aufgabe Privaten überlassen, oder ob sie eine Aufgabe in eigener Regie durchführen.

Die Systematisierungsversuche WAGENERS ${ }^{1}$ ) und der KOMMUNALEN GEMEINSCHAFTSSTELLE FOR VERWALTUNGSVEREINFACHUNG (KGSt) ${ }^{2}$ ) stellen insofern eine Erweiterung dar, da sie den vorhandenen Aufgabenbestand nach staatlichen Aufgabenträgern ordnen und damit Auskunft über Verflechtungen und Kompetenzregelungen zwischen den staatlichen Organisationseinheiten geben. Da es diesen Systematiken jedoch ebenfalls an einem analytischen konzept mangelt, ${ }^{3}$ ) liefern auch sie keine Unterscheidungskriterien für eine deskriptive Abgrenzung öffentlicher von privaten Aufgaben.

1) Vgl. F. Wagener, Einwohnerzahl und Aufgabenerfüllung der Länder, in: Materiatien zum Bericht der Sachverständigenkommission für die Neugliederung des Bundesgebietes, hrsg. vom Bundesministerium des Innern, Bonn 1973, S. $84 \mathrm{ff}$.

2) $\mathrm{Vgl}$. Kommunale Gemeinschaftsstelle für Verwaltungsvereinfachung (KGSt), System der öffentlichen Aufgaben, Pilotstudie, Köln 1974.

3) So auch G. F. Schuppert, Die öffentliche Aufgabe als Schlüsselbegriff der Verwaltungswissenschaft, a.a.0., S. 325. 


\subsection{Aufgabengliederungen nach materiellen Kriterien}

Weitergehender sind Systematisierungsversuche, die die Aufgaben nicht lediglich nach formalen Gesichtspunkten ordnen, sondern sie materiell verschiedenen Funktionsbereichen zuweisen. In der Literatur sind zahlreiche Aufgabensystematiken dieser Art zu finden, wobei die zugrundegelegten Gesichtspunkte, nach denen die Aufgabenbereiche separiert werden, mit den unterschiediichen Untersuchungsgegenständen und dem jeweiligen Erkenntnisinteresse variieren:

- ELLWEIN ${ }^{1)}$ nimmt eine Grobgliederung der Aufgabenbereiche nach Bürgernähe vor. Er unterscheidet zwischen Aufgaben der Politik, die den Bürger unmittelbar und damit die ordnung der Gesellschaft betreffen (Formulierung, Begrenzung und Schutz der persönlichen und politischen Rechte und Freiheiten; Förderung der persönlichen Entfaltung; soziale Sicherung), Aufgaben der Politik, die Raum und Wirtschaft betreffen (u.a. allgemeine Strukturaufgaben, Erwerbsförderung und Geldwesen) sowie Aufgaben der Politik, die das für die öffentlichen Aufgaben notwendige Instrumentarium betreffen (z.B. Verwaltungsorganisation, öffentliche Einrichtungen und öffentlicher Dienst).

- Nach dem Gehalt (der Qualität) der verwaltenden Tätigkeit gliedern WOLFF und BACHOF ${ }^{2}$ ) die Bereiche der öffentichen Verwaltung in ordnende, leistende, Abgaben- und Bedarfsverwaltung.

- SCHARPF ${ }^{3}$ ) unterscheidet staatsfunktionen erster bis dritter Ordnung und gliedert diese nach der Intensität ihres Eingriffs und - deckungsgleich damit - nach der zeitlichen Reihenfolge ihrer Entwicklung in der kapitalistischen Gesellschaft. Die Staatsfunktionen erster Ordnung umfassen die traditionellen Aufgabenbereiche wie Gewährleistung äußerer und innerer Sicherheit sowie die Einrichtung einer berechenbaren und sanktionierten Rechts- und Vertragsordnung. Zu den Staatsfunktio-

1) Vgl. Th. Ellwein, Einführung in die Regierungs- und Verwaltungslehre, a.a.0., S. 36 .

2) Vgl. H. J. Wolff und 0. Bachof, Verwaltungsrecht I, 9. Aufl., München 1974, § 3 Ib (5. 18).

3) Vgl. F. W. Scharpf, Planung als politischer Prozeß, Frankfurt/M. 1973, S. $16 \overline{3 \mathrm{ff} \text {. }}$ 
nen zweiter Ordnung zählt SCHARPF alle jene, die der Beeinflussung des Wirtschaftsprozesses durch die Veränderung globaler Parameter ohne direkten Eingriff in die individuellen Produktionsentscheidungen der Unternehmen dienen (z.B. Infrastrukturvorleistungen und Globalsteuerung). Die direkte staatliche Einflußnahme auf Zielsetzung, Verfahrensweisen und GröBenordnungen ökonomisch-technologischer Entwicklungsprozesse, wie z.B. Produktionsauflagen in der Rustungsindustrie oder regionale und sektorale strukturpolitik, faBt er schließlich zu den Staatsfunktionen dritter ordnung zusammen.

- Ahnlich wie SCHARPF geht auch RICHARD ROSE ${ }^{1)}$ vor. Auch ROSE unterscheidet drei Aufgabenbereiche, die ebenfalls die zeitliche Abfolge ihrer übernahme als Bereiche staticher Aufgabenerfüllung markieren, und zwar übereinstimmend für die von ihm untersuchten über 30 Staaten westlichen Typs. Aus dem Wesen des Staates ergeben sich zunächst sog. "defining activities", ohne die der moderne staat nicht existenzfähig wäre. Zu diesen Aktivitäten gehört der Schutz der territorialen Integrität, die Aufrechterhaltung der inneren Ordnung sowie die Ausstattung mit finanziellen Mitteln (Steuern, Geldwesen). Maßnahmen zur Förderung des wirtschaftlichen Wohlstandes (mobilizing of physical resources) werden anschließend dem zweiten Aufgabenbereich zugeordnet, sozialstaatliche Maßnahmen (providing social benefits) dem dritten Bereich.2)

- Systemtheoretisch orientiert sind die Gliederung der Aufgabenbereiche von MAYNTZ und eine neuere Aufgabengruppierung von ELLWEIN. MAYNTZ ${ }^{3}$ ) unterscheidet in ihrer systematik folgende Aufgabenbereiche: Die Regelung der Beziehungen zwischen einer Gesellschaft und ihrer Umwelt; die Regelung der Beziehungen

1) $\mathrm{Vgl}$.R. Rose, a.a.0., S. $247 \mathrm{ff}$.

2) Vgl. auch G. F. Schuppert, Die öffentliche Aufgabe als Schluisselbegriff der Verwaltungswissenschaft, a.a.0., S. 314 sowie R. Mayntz, Soziologie der öffentlichen Verwaltung, Heidelberg und Karlsruhe 1978, S. $45 \mathrm{f}$.

3) R. Mayntz, Soziologie der öffentlichen Verwaltung, a.a.0., S. 44. 
unter den Systemmitgliedern; die Sicherung der Handlungsfähigkeit des politisch-administrativen systems; Versorgungsund Dienstleistungen sowie die Steuerung der gesellschafti chen Entwicklung auf bestimmte Ziele hin. Indem er den Stat als System und die Aufgaben als Funktionen dieses Systems betrachtet, unterscheidet ELLWEIN ${ }^{1}$ ) zwischen Ordnungsfunktionen, Funktionen, die das system verteidigungsfähig machen, Aus gleichs- und Dienstleistungsfunktionen sowie gestaltenden Funktionen.

- Weiter differenziert ist schließlich die Aufgabensystematik BULLS. 2) Er leitet aus der Verfassung fünf grobe Aufgabenbereiche $a b$, die dann weiter konkretisiert werden (vgl. Obersicht 4). In seinen theoretischen Grundlagen weist BULL zuvor nach, daß sich aus dem Sozialstaatsprinzip und aus dem veränderten Verständnis der Grundrechte eine Ausweitung der staat1 ichen Aufgaben ergeben. ${ }^{3)}$ Aus der Betonung des Sozialstatsprinzips folgt bei BULL zwangsläufig eine spezielle - allerdings nicht unkritisiert gebliebene 4 ) - Rangeinstufung der Aufgabenbereiche, welche die Gewährleistung von Sicherheit und Ordnung hinter den Leistungsaufgaben zurückstehen 1 äßt.

Die Auswahl der Aufgabensystematiken verdeutlicht, daß eine Zuordnung der Aufgaben zu unterscheidbaren Funktionsbereichen grundsätzlich nach sehr verschiedenen Gesichtspunkten möglich ist. Kritisch ist allerdings auch hier zunächst anzumerken, daß die Systematiken nicht in gleichem Maße konsistent wirken. Nicht in allen Fällen ist eine Grenzlinie zwischen den einzelnen Funktionsbereichen klar und eindeutig gezogen worden. Dies gilt beispielsweise für die von ELLWEIN vorgenommene Trennung in Aufgaben, die den Bürger unmittelbar und solche, die den Bürger lediglich mittelbar über Einwirkungen auf Raum und Wirtschaft be-

1) Vgl. Th. Ellwein, Das Regierungssystem der Bundesrepublik Deutschland, a.a.0., S. $83 \mathrm{f}$.

2) Vgl. H.P. Bul1, a.a.0., $\S \S 14-18$ (S. 224 f.).

3) Ebenda, $\S 11$, insb. S. $176 \mathrm{ff}$.

4) Siehe dazu R. Hillmann und H. von Wersebe, a.a.0., S. 96. 
1. SICHERUNG DER EXISTENZGRUNDLAGEN DES EINZELNEN

- natuirliche Grundlagen des menschlichen Lebens (Umweltschutz)

- wirtschaftliche Sicherheit des einzelnen (Sozialpolitik, Wohlfahrt)

- weitere Daseinsvorsorge (Ver- und Entsorgung, Verkehr, Straßen- und Wohnungsbau)

- Gesundheitsfürsorge

2. SICHERUNG DER WIRTSCHAFTLICHEN GRUNDLAGEN DES GEMEINWESENS

- allgemeine Wirtschaftspolitik (Produktivität, hoher Lebensstandard, Vollbeschäftigung, gerechte Verteilung, Wachstum, Preisstabilität, außenwirtschaftliches Gleichgewicht, Krisenvorbeugung)

- Steuer- und Lohnpolitik

- besondere Wirtschaftspolitik (Infrastrukturpolitik, Währungswesen, Förderung einzelner Wirtschaftszweige)

- wirtschaftliche Eigenbetätigung des Staates

3. SICHERUNG DER MENSCHLICHEN ENTFALTUNGSMÖGL ICHKEITEN

- Ausbildung (Schule, Hochschule, Forschung)

- Weiterbildung

- Kultur, Unterhaltung, Sport

- staatliche Erziehungsaufgaben (Familien- und Jugendhilfe, Strafvollzug)

4. ORDNUNG DES ZUSAMMENLEBENS

- durch Rechtsetzung (auch soziale und technische Normierung)

- durch positive Leistungen des Staates (Register, Verkehrsregelung)

5. GEWAHRLEISTUNG DER ORDNUNG

- Schutz des einzelnen (Gefahrenvorsorge, Polizei, Feuerwehr etc.)

- Schutz der Rechtsordnung im übrigen (Rechtspflege, Vollstreckung)

- Staatsschutz (Verfassungsschutz etc.)

- äußere Sicherheit (Außen-, Verteidigungs-, Entwicklungspolitik)

treffen. 1) Nicht immer ist es darüber hinaus gelungen, eine Vermischung von Primär- und Sekundärfunktionen zu vermeiden. Indem die eigentlichen Aufgabenbereiche und die Instrumente zur Erfuillung dieser Aufgaben gleichrangig nebeneinander gestellt werden,

1) Vgl. Th. Ellwein, Einführung in die Regierungs- und Verwaltungslehre, a.a.0., S. 36. Kritisch dazu auch H. P. Bull, a.a.0., S. 215. 
leiden die Systematiken vielfach unter einer mangelhaften Homogenität. Besonders auffällig geschieht diese Verquickung wiederum bei ELLWEIN, indem er als dritte Kategorie einen Aufgabenbereich ausweist, der das für die Erfüllung der in den ersten beiden Kategorien genannten öffentlichen Aufgaben erforderliche Instrumentarium betrifft. 1) Aber auch WOLFF und BACHOF stellen die eher Hilfsfunktionen erfüllende Abgaben- und Bedarfsverwaltung auf eine Ebene mit der ordnenden und leistenden Verwaltung. Erneut wird hier die bereits diskutierte Ziel-Mittel-Problematik deutlich.

Vielfach wird mit den systematisierungsversuchen gar nicht primär das Ziel einer Aufgabenabgrenzung zwischen öffentlichem und privatem Sektor verfolgt, sondern sie haben vornehmlich ganz andere Zwecke zu erfüllen, nämlich den als Resultat des politischen Prozesses feststellbaren Bestand öffentlicher Aufgaben - teilweise sogar nur der staatlichen Aufgaben oder der Verwaltungsaufgaben - ex post anhand mehr oder weniger plausibler Kriterien nach ihren Funktionen zu ordnen. Private Aufgaben ergeben sich dabei als Restgröße der als öffentlich identifizierten Funktionen. Eindeutige Kriterien, mit deren Hilfe das "eigentlich offentliche" vom "spezifisch Privaten" separiert werden könnte, lassen sich daraus jedenfalls nicht gewinnen. Die Systematisierungsversuche sind zudem zwangsläufig - wenn auch in unterschiedlichem Ausmaß - geprägt von einem bestimmten subjektiven Vorverständnis ihrer Autoren über öffentliche Aufgaben, d.h. ihre Aufgabengruppierungen enthalten mehr oder weniger normative Elemente und gehen somit über eine reine Beschreibung öffentlicher Aufgaben zum Teil erheblich hinaus.

1) Vgl. Th. Ellwein, Einführung in die Regierungs- und Verwaltungslehre, a.a.0., S. 36 .

2) Vgl. H. J. Wolff und 0 . Bachof, Verwaltungsrecht I, a.a.0., 33 Ib (S. 18). 
3. Verwirklichung des Gemeinwohls versus Maximierung des Eigennutzens

\subsection{Eingrenzung der Diskussion}

Ein alternativer Versuch der funktionalen Abgrenzung öffentlicher von privaten Aufgaben besteht darin, "Gemeinwohl" bzw. "öffentliches Interesse"1) zum Entscheidungskriterium für eine Aufgabenabgrenzung zu erheben. Dieser Ansatz leitet sich aus der organischen staatsauffassung ab und wurde insbesondere in der früheren Finanzwissenschaft verwand ${ }^{2}$ ), aber auch heute spielt er in Politik und Wissenschaft noch eine erhebliche Rolle ${ }^{3}$ ) und findet ebenfalls in der Rechtsprechung oberster Gerichte seinen Niederschlag. ${ }^{4}$ )

Zugrundegelegt wird hier die Vorstellung, private Aufgaben seien vornehmlich durch die Erfiullung von Einzelinteressen gekennzeichnet, sie dienten somit der individuellen Nutzenmaximierung, während öffentliche Aufgaben primär an gesellschaftlichen Zielen orientiert seien und damit im Dienste des Gemeinwohls bzw. im öffentlichen Interesse erfolgten.

Die Diskussion um das Gemeinwohl und um das öffentliche Interesse leidet erheblich darunter, daß beide Termini ausgesprochen häufig Verwendung finden, ohne daß damit zugleich exakte Vorstellungen über ihre inhaltliche Ausgestaltung verbunden werden. Ihre Beliebtheit ergibt sich offenbar gerade aufgrund ihrer vielfältigen Verwendungsmöglichkeiten, insbesondere aufgrund der Tatsache, daß sich auch diametral entgegengesetzte politische

1) Das öffentliche Interesse stellt anders als das Gemeinwohl in erster Linie eine jurisiische Kategorie dar. Nicht selten werden beide $\mathrm{Be}$ griffe aber auch synonym verwendet, oder es wird lediglich aus sprachlichen Gründen einer der beiden Ausdrücke vorgezogen; so z.B. P. Häberle, offentliches Interesse als juristisches Problem, Bad Homburg v.d.H. 1970, S. 22 .

2) Einen kritischen Abriß der wichtigsten Positionen unternimmt $K$. Schmidt, Zur Geschichte der Lehre von den Kollektivbediurfnissen, a.a.0., S. $335 \mathrm{ff}$.

3) Einen überblick geben C. Böhret, W. Jann, M.T. Junkers, E. Kronenwett, Innenpolitik und politische Theorie, a.a.0., insbesondere Kap. 10, S. $309 \mathrm{ff}$.

4) Vgl. exemplarisch BVerfGE 50, S. 29, S. 51, S. 55, S. 114, S. 178 , S. 216 und S. 259 sowie BVerfGE 51, S. 176 und S. 408. 
Konzeptionen auf das Gemeinwohl berufen können bzw. vorgeben können, im öffentlichen Interesse zu handeln. Dieser Umstand steigert jedoch allenfalls ihren Wert für ideologische Auseinandersetzungen. 1)

Es würde den Rahmen dieser Arbeit sprengen, die breite und stark verästelte Gemeinwohldiskussion auch nur in groben Zügen nachzeichnen zu wollen. ${ }^{2)}$ Dieses ist auch nicht erforderlich, vielmehr erscheint eine Eingrenzung der Diskussion in doppelter Hinsicht angebracht.

Erstens: Dem Terminus Gemeinwohl haften bereits stark normative Züge an. Gleichwohl kann in diesem Kapitel auf allgemeine Oberlegungen normativer Art im Hinblick auf eine inhaltiche Fassung des Gemeinwohlbegriffes genauso verzichtet werden wie auf spezielle wirtschaftstheoretische Versuche, ein ökonomisches Gemeinwohl normativ zu umschreiben, d.h. unter verwendung einer gesellschaftlichen Wohlfahrtsfunktion das Ziel der Wohlstandsmaximierung für die gesamte Volkswirtschaft zu formulieren und die Bedingungen abzuleiten, unter denen dieses Maximum zu rea1 isieren ist.

Zweitens: Die verschiedenen Standpunkte im Hinblick auf die Verwirklichung des Gemeinwohls werden allein daraufhin analysiert, ob und inwieweit die dort zum Ausdruck kommenden Auffassungen als geeignete Kriterien für eine Abgrenzung privater von öffent-

1) Allerdings stellt - für die Rechtswissenschaft wie für die Rechtsprechung das öffentliche Interesse einen zwar unbestimmten, aber dennoch unentbehrlichen Rechtsbegriff dar. Lakonisch bemerkt hierzu Leisner: "... die Begeisterung über diesen Pyrrhussieg vergißt aber, daß an dem 'Rechtsbegriff' nichts bemerkenswert ist, als seine Unbestimmtheit," W. Leisner, Privatinteressen als öffentliches Interesse, DOV 23. Jg. (1970), S. 221.

2) Statt Vieler vgl. deshalb aus ökonomischer Sicht besonders G. Colm, In Defense of the Public Interest, in: Social Research, 27. Jg. (1960), S. $295 \mathrm{ff}$. Sowie Th. Puitz, Das "Gemeinwohl" als Begriff der theoretischen Wirtschaftspolitik, in: Zeitschrift für Nationalökonomie, 26 . Jg. (1966), S. $260 \mathrm{ff}$.; aus juristischer Sicht vor allem $P$. Häberle, offentliches Interesse als juristisches Problem, a.a.0.; des weiteren G. Schubert, The Public Interest, Glencoe, III. 1960, der einen kritischen Oberblick über verschiedene Gemeinwohlkonzeptionen vermittelt; zur interdisziplinären Erörterung $v g l$. C. J. Friedrich (Hrsg.), The Publ ic Interest, Nomos Bd. 5, New York 1962; überwiegend interdisziplinär angelegt ist auch die Untersuchung von $\underline{H}$.H. von Arnim, Gemeinwohl und Gruppeninteressen, Frankfurt/M. 1977. 
lichen Aufgaben angesehen werden können. Eine positive Antwort ist wiederum an folgende Voraussetzungen geknüpft:

- Es muß der Nachweis geführt werden, daß ein Gemeinwohl existent und operationalisierbar ist, insbesondere muß auch deutlich werden, wer als Träger von Gemein- und Einzelinteressen fungiert.

- Es muß eine klare Trennung zwischen Aktivitäten, die dem Gemeinwohl dienen, und Handlungen, die aus dem Eigennutzmotiv hervorgehen, durchführbar sein.

\subsection{Zur Identifikation des Gemeinwohls in bipolaren Staats- auffassungen}

\subsubsection{Organische Staatsauffassung}

Trotz der verzweigten und auf verschiedene Nebenschauplätze verlagerten Diskussion können zwei grundsätzliche und polare Staatsauffassungen identifiziert werden. Gemäß der organischen Staatstheorie gibt es ein a priori erkennbares und inhaltich allgemeingïltig faBbares Gemeinwohl. Dieses Gemeinwohl kann nur der Staat, der als den Individuen uibergeordnetes Ganzes begriffen wird, realisieren. Der Staat verkörpert also das Gesamtinteresse, welches offenbar nicht gleichzusetzen ist mit der summe der Einzelinteressen. " Aus dem Auseinanderklaffen von individuellen Präferenzen und dem vom Staat erkannten Gemeinwohl resultieren dann auch öffentliche Aufgaben, d.h. konkret die öffentliche Bereitstellung von Gütern, die nicht von einzelnen Wirtschaftssubjekten nachgefragt werden.

Besondere Bedeutung kommt innerhalb der organischen Staatsauffassung der Frage zu, durch wen und auf welchem Wege die Artikulation des Gemeinwohls erfolgt. Während vor allem ältere Finanzwissenschaftler wie VON HERMANN, MENGER und SCHÄFLE noch die Meinung vertraten, daß der Stat bzw. die Gesellschaft als solche Träger des Gemeinwohls sei und es auch formulieren

1) So Z.B. auch H. Krüger, Allgemeine Staatslehre, 2. Auf1., Stuttgart 1966, S. 765; vgl. dazu auch H. H. von Arnim, a.a.0., S. 9 f. 
könne ${ }^{1)}$, besteht heute Einigkeit darüber, daß sämtliche Versuche, aus einer Bedürnisse empfindenden Gemeinschaft öffentliche Aufgaben ableiten zu wollen, fehlgeschlagen sind. Bedürfnisse und Interessen können allein von Individuen geäußert werden, "since the group as such cannot speak."2) Alles andere birgt die Gefahr, daß man sich ins Transzendentale begibt, der Staat mithin zu einem "corpus mysticum" 3 ) verklärt wird.

Als Ausweg bleibt der organischen Staatstheorie dann nur die Anerkennung einer Elite oder Führungsgruppe, die bewußt in die Konsumentensouveränität eingreift, d.h. es sind dann vornehmlich die Politiker, die ihre Präferenzen zu Gesamtinteressen erklären und somit auch über Art und Umfang öffentlicher Aufgaben bestimmen.

\subsubsection{Individualistische Staatsauffassung}

Die Gegenposition leugnet die Möglichkeit einer ex ante-Bestimmung des Gemeinwohls. Bereits seinem Wesen nach läßt der Begriff eine allgemeingultige Definition nicht zu. Losgelöst von konkreten Verhältnissen stellt er lediglich eine Leerformel dar, die jederzeit beliebig ausgefillt werden kann. Öber die jeweilige konkrete inhaltliche Ausgestaltung des Gemeinwohls wird im politischen Prozeß entschieden. Anders als im Rahmen der organischen Staatstheorie manifestiert sich hier das Gemeinwohl als ein Kompromiß zwischen den vielschichtigen, sich teils unterstuitzenden, teils miteinander konkurrierenden Individual- und Gruppeninteressen der an dem politischen Prozeß Beteiligten. Genauso wie das Ergebnis des politischen Prozesses ist damit aber auch der Inhalt des Gemeinwohls ständigen Wandlungen unterworfen.

1) Vgl. K. Schmidt, Zur Geschichte der Lehre von den Kollektivbedürfnissen, a.a.0., S. $335 \mathrm{ff}$.

2) R. A. Musgrave, The Theory of Public Finance, a.a.0., S. 87. Auf diese Tatsache hat bereits Jecht 1928 hingewiesen; vgl. H. Jecht, Wesen und Formen der Finanzwissenschaft, Jena 1928, S. 62.

3) K. Schmidt, Zur Geschichte der Lehre von den Kollektivbedürfnissen, a.a.0., S. 338 . 
In ihrer strengen Form beruht die individuelle staatsauffassung auf Prinzipien, die - in einem mehr technischen Sinne - überhaupt als Grundlagen des methodologischen Individualismus gelten. Das Individuum bildet den Ausgangspunkt für alles Handeln. Nur es allein ist Träger von Interessen und Bedürfnissen. Das einzelne Wirtschaftssubjekt kennt seine persönlichen Präferenzen besser als jeder staatliche Funktionär. Es ist in der Lage, diese Präferenzen in eine Rangordnung zu bringen und so seinen individuellen Nutzen zu maximieren. Das Individuum entscheidet autonom, d.h. es besteht Konsumenten-bzw. Wählersouveränität. Auch kollektive Handlungen sind das Ergebnis individueller Präferenzen. Dem Staat fällt deshalb lediglich die Aufgabe zu, dafür zu sorgen, daß diese individuellen Präferenzen der Wirtschaftssubjekte durchgesetzt werden können. Ein Eigeninteresse des Staates, verbunden etwa mit einem Eingriff in die Präferenzstruktur der Individuen, wird abgelehnt.

Häufig wird in diesem Zusammenhang angeführt, im Rahmen der individualistischen Staatsauffassung ergebe sich das Gemeinwohl als Summe der Einzelinteressen. Aufgrund unpräziser Argumentation resultieren hieraus allerdings häufig Mißverständnisse. Zwar gilt im Gegensatz zur organischen Staatstheorie, daß das Gemeinwohl stets auf individuelle Vorstellungen zurückzuführen ist, wie aber bereits ARROW mit Hilfe des Unmöglichkeitstheorems bewiesen hat, ist es jedoch u.a. wegen der Unzulässigkeit interpersoneller Nutzenvergleiche nicht möglich, aus den individuellen Präferenzen, welche die Wirtschaftssubjekte auf Grundlage einer ordinalen und daruber hinaus konsistenten und transitiven Präferenzskala in eine Rangfolge bringen, eine gesellschaftliche Wohlfahrtsfunktion abzuleiten, ohne zugleich eine der Be- 
dingungen des individualistischen Prinzips zu verletzen. 1) Im strengen Wortsinn kann das Gemeinwohl deshalb nicht als die Summe der Einzelinteressen verstanden werden.

Wird die individualistische Staatsauffassung zugrundegelegt, die MUSGRAVE als allein kompatibel mit einem demokratischen system erklärt ${ }^{2}$, so ist offenbar eine exakte Trennung von Gemeinwohl und individuellem Nutzenkalkül nicht mehr ohne weiteres durchführbar. Vielmehr wird davon ausgegangen, daß das Streben nach persönlicher Nutzenmehrung mit dem Gemeinwohl harmoniert. Für den Begründer des ökonomischen Liberalismus, ADAM SMITH, fallen Eigen- und Gesamtinteresse sogar völlig zusammen. Durch die "List der Vernunft" wird unter den Bedingungen des freien Wettbewerbs der Einzelne seine Fähigkeiten so einsetzen, daß er einen möglichst hohen Ertrag erzielt. Indem er danach trachtet, seine egoistischen Ziele durchzusetzen, maximiert er gleichzeitig unbewußt den Nutzen für die gesamte Volkswirtschaft. "Und er wird in diesem wie auch in vielen anderen fällen von einer unsichtbaren Hand geleitet, um einen Zweck zu fördern, den zu erfiillen er in keiner Weise beabsichtigt hat."3)

1) Arrow formulierte folgende fünf Bedingungen, die erfültt sein müssen, wenn bei Aufrechterhaltung des individualistischen Konzepts die Ableitung einer gesellschaftlichen Wohlfahrtsfunktion aus den Präferenzen der einzelnen Wirtschaftssubjekte möglich sein soll:

1. Für sämtliche Alternativen ist jede denkbare individuelle Rangordnung zulässig.

2. Zieht jedes Wirtschaftssubjekt eine Alternative $A$ der Alternative $B$ vor, so soll dies auch für die Gesellschaft gelten.

3. Die gesellschaftliche Präferenzskala wird ausschließlich durch die individuellen Präferenzordnungen bestimmt (Unabhängigkeit von irrelevanten Alternativen).

4. Die Wirtschaftssubjekte sind in ihren Entscheidungen souverän.

5. Kein Wirtschaftssubjekt ist in der Lage, seine individuelle Präferenzordnung zur gesellschaftlichen Präferenzordnung zu erheben (Ausschluß der Diktatur).

Vgl. K. J. Arrow, Social Choice and Individual Values, 2. Aufl., New York 1963, S. $22 \mathrm{ff}$. Das Theorem selbst formuliert er auf S. 59, der Bewe is erfolgt auf S. $97 \mathrm{ff}$.

2) Vgl. R. A. Musgrave, The Theory of Public Finance, a.a.0., S. 87.

3) A. Smith, Der Wohlstand der Nationen. Eine Untersuchung seiner Natur und seiner Ursachen. Aus dem Englischen übertragen und mit einer Würdigung von H. C. Recktenwald, München 1974, S. 371. 
Die von SMITH dargelegten, auf den Vorstellungen des methodologischen Individualismus beruhenden, Verhaltensweisen der im marktwirtschaftlichen Prozeß agierenden Unternehmer und Konsumenten übertragen 180 Jahre später, aufbauend auf den von SCHUMPETER ${ }^{1)}$ in diesem Zusammenhang entwickelten Grundideen, DOWNS $^{2)}$ und HERDER-DORNEICH ${ }^{3)}$ auf den politischen Prozeß. Die Wähler verhalten sich analog zu den Konsumenten am Markt als Nutzenmaximierer. Gemäß ihren Präferenzen entscheiden sie sich fur das politische Programm - hier verstanden als aggregiertes Güterbiundel - derjenigen Partei, welches ihnen den höchstmöglichen Nutzen verspricht. In gleicher Weise wie die Marktunternehmer danach trachten, ihren Gewinn zu maximieren, streben die Politiker - quasi als politische Unternehmer - nach Stimmenmaximierung. Nunmehr ist es nicht mehr die Sorge um das Gemeinwohl, das den Politiker in seinem Tun beflügelt, sondern sein Handeln wird geprägt durch egoistische Motive wie hohes Ansehen, Einfluß, Einkommensverbesserung etc. Unter den Bedingungen eines funktionierenden Wettbewerbs zwischen den Politikern ist es analog zum Marktmechanismus, wo die unsichtbare Hand trotz des Ejgennutzstrebens der Individuen das gesamtwirtschaftliche Wohl herbeiführt, hier nun die "List der Demokratie"4), die dafür sorgt, daß in Verfolgung der Einzelinteressen gleichzeitig dem Gesamtinteresse gedient wird.

Sowohl bei ADAM SMITH als auch bei DOWNS und HERDER-DORNEICH werden also Individual- und Gesamtinteresse deckungsgleich. Eine Trennung zwischen beiden Größen wird dadurch unmöglich. Damit leistet dieses Kriterium aber auch nichts mehr für eine Aufgabenabgrenzung.

1) Vgl. J.A. Schumpeter, Kapitalismus, Sozialismus und Demokratie, Bern 1946 , S. $247 \mathrm{ff}$.

2) Vgl. A. Downs, An Economic Theory of Democracy, New York 1957. (Deutsche Ubersetzung: Okonomische Theorie der Demokratie, Tübingen 1968).

3) Vgl. Ph. Herder-Dorneich, Politisches Modell zur Wirtschaftstheorie, Freiburg 1959, S. $41 \mathrm{ff}$. (Eine Teilauflage dieses Buches ist unter dem Pseudonym F. O. Harding erschienen).

4) Vgl. Ph. Herder-Dorneich, a.a.0., S. 57. 
Die Tatsache, daß es in der Realität zu Abweichungen von diesen idealtypischen Modellen kommen kann, wird allerdings von keinem der Autoren geleugnet. Die Skepsis gegenüber einer vorbehaltiosen Gleichsetzung von Einzel- und Gesamtinteresse kommt bei

SMITH insbesondere dort deutlich zum Ausdruck, wo er sich äuBerst kritisch mit den Verhaltensweisen mancher Unternehmer auseinandersetzt, ihnen Habgier und Monopolgeist vorwirft und resumiert: "Ihr Interesse ist, in dieser Hinsicht, dem des überwiegenden Teils der Bevölkerung genau entgegengesetzt."1) Auch DOWNS kann sich nicht dazu entschließen, das Gemeinwohl völlig aus seiner Analyse zu verbannen. 2) Das gleiche gilt auch für MUSGRAVE, wenn er sich zur individualistischen Staatsaufiassung bekennt, diesen methodischen Ansatz aber nicht durchzuhalten vermag. Indem er es für legitim hält, daß in einer Demokratie zuweilen eine informierte Gruppe ihre Vorstellungen gegenüber den abweichenden Präferenzen der weniger informierten Individuen durchsetzt ${ }^{3)}$, erkennt er damit implizit ebenfalls die Existenz eines Gemeinwohls an. Etwas belustigt stellt deshalb COLM fest, daB die Theoretiker des Eigeninteresses gelegentlich "permit the public interest a kind of incognito entrance through the back door." 4 )

1) A. Smith, a.a.0., S. 407. Nicht nur an dieser Stelle wird deutlich, daB Smith selbst weder für ein schrankenloses Laissez-faire eintrat noch von einem blinden Harmonieglauben geleitet wurde, wie ihm dies in zahlreichen Veröffentlichungen unterstellt wurde und heute noch wird, sondern da $B$ er wesentlich differenzierter das Selbstinteresse der Wirtschaftssubjekte durch rechtliche Normen begrenzt sah und einen Egoismus ablehnte, der auf Kosten anderer durchgesetzt wird. Vgl. hierzu auch E. Tuchtfeldt, ober die Staatsfunktionen be i Adam Smith, in: Ordo, 27. Jg. (1976), S. $37 \mathrm{f}$. sowie E. Wille und M. Gläser, Staatsaufgaben bei Adam Smith - eine Wirdigung unter AlTokationsaspekten, in: Ordo, 26. Jg. (1977), S. $34 \mathrm{ff}$.

2) So weist er z.B. darauf hin, daß in einigen Fällen staatliche Eingriffe unbestreitbar gute Reallokationen herbeiführen, zu denen der Markt nicht in der Lage ist; A. Downs, a.a.0., S. 173.

3) Vgl. R. A. Musgrave, The Theory of Public Finance, a.a.0., S. $13 \mathrm{f}$.

4) G. Colm, In Defense of the Public Interest, a.a.0., S. $296 \mathrm{f}$. 


\subsection{Schlußfolgerungen}

Die kurzen Hinweise erlauben bereits Schlußfolgerungen: Abgesehen von der Skepsis gegenüber den Möglichkeiten einer objektiven ex ante-Bestimmung des Gemeinwohls, leidet die Diskussion im Rahmen der organischen Staatstheorie insbesondere unter dem - methodischen - Mangel, daß sie einen Gegensatz zwischen Eigen- und Gesamtinteresse konstruiert, der nicht zu belegen ist. Wie viele Beispiele zeigen, kann ein Verhalten der individuellen Nutzenmehrung genauso mit dem Gemeinwohl in Einklang stehen, wie umgekehrt Maßnahmen, die dem Gemeinwohl gewidmet sind, grundsätzlich einen individuellen Nutzenzuwachs hervorrufen können. 1) Die individualistische Staatsaufassung vermeidet zwar diese strikte Trennung zwischen Eigennutz und Gemeinwohl, aber ihr Konzept der alleinigen Ausrichtung des statichen Handelns nach den Präferenzen der Individuen ist eine ähnliche Fiktion wie die Konsumentensouveränität im Marktbereich. Die organische Staatsauffassung erkennt dagegen zumindest an, daß Aktivitäten des Staates auch gegen die Interessen des Individuums gerichtet sein können. Eine konsequente und überzeugende Anwendung der Kriterien "Gemeinwohl" bzw. "öffentliches Interesse" zur Abgrenzung privater und öffentlicher Aufgabenbereiche gelingt jedenfalls mit keinem der beiden Konzepte.

\section{Erkenntniswert funktionaler Abgrenzungskriterien}

Die ökonomische Relevanz einer Aufgabenabgrenzung anhand funktionaler Kriterien ist unbestritten: Könnte nämlich das Gemeinwohl ex ante objektiv bestimmt werden und daruber hinaus alle Tätigkeiten, die zur Realisierung des Gemeinwohls erfolgen, unzweifelhaft von jenen Aktivitäten, die der individuellen Nutzenmehrung dienen, separiert werden, so wäre ein eindeutiges Kriterium gewonnen, um die öffentlichen und privaten Aufgaben in

1) Ein hervorragendes Beispiel bieten in diesem Zusammenhang die Aktivitäten des beliehenen Unternehmers, ist es doch gerade Absicht der Beleihung, privates Engagement für öffentliche Belange nutzbar zu machen; so auch W. Leisner, Privatinteressen als öffentliches Interesse, a.a.0., S. 219. 
einer Volkswirtschaft nicht nur formal, sondern materiell unterscheiden zu können. Es wurde jedoch gezeigt, daß bereits die erste Voraussetzung nicht erfült ist. Der konkrete Inhalt des Gemeinwohls ergibt sich allenfalls ex post als Resultat des politischen Prozesses, d.h. die Identifikation des "eigentlich offentlichen" und des "spezifisch Privaten" verbleibt im Nebulösen einer "black box". Der Erkenntniswert einer Deskription öffentlicher und privater Aufgaben mittels funktionaler Abgrenzungskriterien ist deshalb als außerordentlich gering zu veranschlagen. Dies gilt umso mehr, da Versuche, weitgehend unbestrittene öffentliche Funktionen zu benennen, die einen sog. "öffentlichen Kernbereich" bilden, oder öffentliche Aufgaben anhand funktionaler Gesichtspunkte systematisch zu ordnen, ebenfalls keinen adäquaten Beitrag zu einer deskriptiven Aufgabenabgrenzung leisten.

\section{Institutionelle Abgrenzungskriterien}

Eine weitere Möglichkeit der Abgrenzung öffentlicher von privaten Aufgaben besteht offenbar in der einfach anmutenden Beantwortung der Frage nach den Handlungs- oder auch Funktionsträgern der Aufgaben. Was öffentliche bzw. private Aufgaben sind, würde demnach danach beurteilt werden, welche Institutionen bzw. Welche Wirtschaftssubjekte die Erfiullung der Aufgaben übernehmen. Von einer öffentlichen Aufgabe wäre demzufolge immer dann zu sprechen, wenn sie von einem öffentlichen Träger wahrgenommen wird, eine private Aufgabe läge hingegen stets in jenen Fällen vor, in denen ein privater Träger aktiv wird. Mit Hilfe der Trägerschaft wäre damit ein formales Kriterium gewonnen, welches die exakte Zuordnung von Aufgaben zum öffentlichen resp. privaten Sektor der Volkswirtschaft erlaubt. Die Frage nach den konstitutiven Kriterien öffentlicher Aufgaben wird auf diesem Wege also transformiert in die Frage nach den grundlegenden Charakteristika öffentlicher Aufgabenträger. 
1. Zur Notwendigkeit einer Uberwindung der Dichotomie von Markt und Staat

Die in der Wirtschaftswissenschaft vorherrschende Betonung funktionaler Zusammenhänge hat dazu geführt, daß in der Vergangenheit Fragen nach dem Grenzverlauf zwischen privatem und öffentlichem Sektor überwiegend verkürzt anhand einer Gegenuberstellung von Marktwirtschaft und Staatswirtschaft zu beantworten versucht wurden. üblicherweise wird dann davon ausgegangen, daß der private Sektor in Güter und Dienstleistungen produzierende Unternehmen einerseits und konsumierende private Haushalte andererseits unterteilt werden kann und daß diesem privaten Sektor ein öffentlicher Sektor gegenübersteht, nämlich ein als homogen angesehenes Aggregat "Staat", das Aufgaben wahrnimmt, die von den Wirtschaftssubjekten des privaten Sektors nicht erfüllt werden können oder sollen.

Die Realität ist jedoch unverkennbar dadurch gekennzeichnet, daB zwischen den Polen Markt und Staat ein breiter Bereich liegt, in dem eine Vielzahl höchst heterogener Aufgabenträger mit äußerst unterschiedlichen Zielsetzungen und Handlungsmöglichkeiten tätig wird. Politische Beachtung findet vor allem der Protest- und Forderungscharakter, durch den sich die Aktivitäten vieler Träger von Gruppeninteressen wie z.B. Gewerkschaften, Verbände oder Bürgerinitiativen auszeichnen. Vielfach werden diese Aktivitäten aber gleichzeitig für die ökonomische Analyse relevant, da sie als Träger der Wirtschaftspolitik fungieren ( $z$. B. die gewerkschaftliche Forderung nach Mitbestimmung) und/oder selbst produktive Leistungen erbringen, indem sie Guiter oder Dienste bereitstellen (z.B. Altenhilfe durch caritative Verbände, Erstellung eines Kinderspielplatzes durch eine Bürgerinitiative). 
Auf die Pluralität der Träger der Wirtschaftspolitik wird seit langem hingewiesen. 1) Versuche einer systematischen Erörterung der Fragen, wer eigentlich in der Bundesrepublik Wirtschaftspolitik macht, wie die Einwirkungsmöglichkeiten der Träger der Wirtschaftspolitik strukturiert werden können und welche Entscheidungskriterien für eine optimale organisation des wirtschaftlichen Trägersystems von Bedeutung sind, werden dagegen erst in jüngster Zeit unternommen. ${ }^{2}$ )

Der zweite Aspekt, daß auch die Bereitstellung von Guitern und Dienstleistungen von einem breiten Spektrum öffentlicher und privater Träger vorgenommen werden kann, ist insbesondere im Rahmen der Privatisierungsdebatte der letzten Jahre auf bereitwilliges politisches und wissenschaftliches Interesse gestoßen. Seit der Wissenschaftliche Beirat beim Bundesministerium der Finanzen 1975 darauf verwiesen hat, daß öffentliche Aufgaben nicht nur von statlichen Institutionen, sondern auch von öffentlichen Organisationen auBerhalb der Staatsverwaltung und darüber hinaus von Privaten erfuiltt werden können und "die sich stets wandelnden technischen und institutionellen Bedingungen ... in manchen Bereichen eine derartige Kooperation zwischen Staat und privatem Sektor heute als zweckmäßig erscheinen lassen" 3 ), wurden in einer Reihe von wissenschaftlichen Untersuchungen die Möglichkeiten einer Erfüllung öffentlicher Aufgaben durch nichtstaatliche Träger wie u.a. private Verbände, freie Berufe und Selbstorganisationen Privater analysiert. ${ }^{4}$ )

1) Vgl. H. Giersch, Allgemeine Wirtschaftspolitik, Erster Band: Grundlagen, Wiesbaden 1961, 5. Kapitel "Macht und Wille wirtschaftspolitischer Instanzen", S. $159 \mathrm{ff} . ;$ B. Rürup, Zur Frage nach den Trägern der Wirtschaftspolitik, ZfgS Bd. $124(1968)$, S. $741 \mathrm{ff}$. Auf internationale und supranationale Träger der Wirtschaftspolitik weist darüber hinaus hin K. G. Zinn, Allgemeine Wirtschaftspolitik, 2. Aufl., Stuttgart u.a. 1974, S. $159 \mathrm{ff}$.

2) Vgl. vor allem K. Kehrle, Rationalität und Effektivität des wirtschaftspolitischen Trägersystems, Frankfurt/M. 1980.

3) Wissenschaftlicher Beirat beim BMF, Zur Lage und Entwicklung der Staatsfinanzen in der Bundesrepublik Deutschland, a.a.0., S. 1007.

4) Vgl. W. Kirberger, a.a.0.; B. Hotz, Politik zwischen Staat und Wirtschaft, Diessenhofen 1979; Studiengruppe für Sozialforschung e.V., Gutachten zu den Bedingungen und MögTichkeiten einer Privatisierung öffent 1 icher Dienstleistungen durch Ubertragung an gebührennehmende Freie Berufe, München 1980; c. Picht, Möglichkeiten einer verstärkten Verlagerung öffentlicher Dienste auf freiberuflich Tätige, Berl in 1984; Ch. Badelt, Sozioökonomie der Selbstorganisation, Frankfurt/M., New York 1980. 
Die Bedeutung solcher - lediglich Teilaspekte berücksichtigender - Abhandlungen sollte in diesem Zusammenhang nicht unterschätzt werden, gleichwohl scheint es unerläßlich, die institutionellen Gesichtspunkte einer Abgrenzung öffentlicher und privater Aufgaben durch eine systematische Erfassung der Aufgabenträger stärker herauszuarbeiten. Die weitere Vorgehensweise kann deshalb wie folgt gekennzeichnet werden:

- In einem ersten Schritt wird der Versuch einer Typologie der Träger der Wirtschaftspolitik sowie der Träger der Bereitstellung von Gütern und Dienstleistungen unternommen, wobei diese zusammengefaßt als Aufgabenträger bezeichnet werden. Zur Unterscheidung öffentlicher und privater Aufgabenträger werden die Kriterien Rechtsform und Eigentumsverhältnisse herangezogen, d.h. es wird davon ausgegangen, daß alle Träger, die im öffentlich-rechtlichen Rahmen agieren, und/oder alle Institutionen, die sich im öffentlichen Eigentum befinden, als öffentliche Träger zu betrachten sind.

- In einem zweiten Schritt wird anschließend die Tauglichkeit dieser Kriterien fur eine Aufgabenabgrenzung sowie ihre ökonomische Relevanz beurteilt, d.h. es wird überprüft, inwieweit eine Kongruenz zwischen den so abgegrenzten öffentlichen Trägern und den öffentlichen Aufgaben einerseits sowie den privaten Trägern und den privaten Aufgaben andererseits feststellibar ist.

\section{Typologie öffentlicher und privater Aufgabenträger}

Eine Typologie der Aufgabenträger muß weder den Anforderungen genügen, die üblicherweise an eine umfassende Begriffsbestimmung gestellt werden, noch erhebt sie den Anspruch, wie eine tiefgegliederte Systematik die Vielzahl der in der Realität existierenden Zwischen- und übergangsformen lückenlos aufzuzeigen. Statt dessen wird mit einer Typologie vor allem das Ziel verfolgt, charakteristische Wesensmerkmale unterscheidbarer Aufgabenträger herauszustellen und zu Typen, die gleiche oder zumindest ähnliche Ausprägungen aufweisen, zusammenzufassen. Damit 
bleibt zwar die typologische Betrachtungsweise zwangsläufig kursorisch, dennoch muB ihr Erkenntniswert darunter nicht leiden, ermöglicht sie doch einen ungetrübten Blick auf die eigentrich bedeutsamen Tatbestände.

Grundsätzlich können zur Typologisierung der Aufgabenträger so verschiedene Wesensmerkmale bzw. Unterscheidungskriterien wie u.a. Zielsetzung, Art der Tätigkeit, Einflußmöglichkeit, Rechtsform und Eigentumsverhältnisse der Träger herangezogen werden. Ein Zirkelschluß wäre die Folge, würden öffentliche Träger wiederum mit Hilfe funktionaler Aspekte, also aufgrund ihrer Zielsetzung oder durch die Art ihrer Tätigkeit gekennzeichnet. Besteht das Anliegen darin, öffentliche Aufgaben gerade mit Hilfe der Eigenschaften ihrer Träger zu bestimmen, so müssen andere Argumente herangezogen werden als jenes, öffentliche Träger zeichneten sich dadurch aus, daß sie öffentliche Aufgaben erfüllen. Als diskriminierende Merkmale sollen deshalb institutionelle Kriterien wie die Rechtsform oder die Eigentumsverhältnisse der Aufgabenträger dienen.

\subsection{Gebietskörperschaften}

Ohne Schwierigkeiten können die zu den Gebietskörperschaften zusammengefaßten Institutionen Bund (einschließlich Lastenausgleichsfonds und ERP-Sondervermögen), Länder sowie Gemeinden den öffentlichen Trägern zugerechnet werden. Aufgrund des formalen Kriteriums der Eigentumsverhältnisse erweist sich eine Zuordnung der Gebietskörperschaften zum öffentlichen Sektor der Volkswirtschaft als ebenso problemlos wie aufgrund der Rechtsform, da sie bereits ex definitione sich sowohl im statlichen Eigentum befinden als auch in einer öffentlich-rechtlichen Organisationsform auftreten. Die Gebietskörperschaften 
werden deshalb auch häufig als "Staat im engeren Sinne" bezeichnet. 1 )

\subsection{Parafiski}

Zu den öffentlichen Trägern können auch jene Einrichtungen gezählt werden, die heute üblicherweise unter dem Sammelbegriff "Parafiski" zusammengefaßt werden. Bereits 1928 hat FRITZ KARL MANN darauf hingewiesen, daß der Staat auch nichtstaatlichen Organen Aufgaben übertragen hat, indem er ihnen die Erhebung von Zwangsbeiträgen gestattet und sie damit zum "Hilfsfiskus" erklärt. Solche "intermediären Finanzgewalten" würden das Staatsbudget entlasten, ohne daß damit eine Reduzieruna der Aufgabenerfüllung verbunden wäre. ${ }^{2)}$ Welche Einrichtungen überwiegend unter dem Begriff "Parafiski" subsumiert werden, zeigt die übersicht 5 .

Da keine allgemeine Definition für den Parafiskus existiert, ist eine Abgrenzung zu den Gebietskörperschaften einerseits und öffentlichen Aufgabenträgern ohne parafiskalische Eigenschaften andererseits schwierig. In der Literatur wird eine Reihe abgrenzender Merkmale genannt, die jedoch unscharf bleiben und je nach Betonung einzelner Charakteristika zu unterschiedlichen Zuordnungen führen. ${ }^{3)}$ Werden die Erzielung von Einnahmen mit steuerähnlichem Charakter durch hoheitlichen Zwang sowie die eigenständige Organisation als Körperschaft des öffentlichen Rechts als additive, konstitutiv wirkende Merkmale des Parafiskus angesehen, so ist zumindest bezüglich der Sozialversicherung und der Berufsvertretungen mit dem er-

1) Während die Zugehörigkeit des Bundes und der Länder zum Sektor Staat al1geme in anerkannt ist, kann die Zuordnung der Gemeinden - vornehmlich unter juristischen Aspekten - nicht mehr so eindeutig beantwortet werden. So spricht die in Art. 28 II GG garantierte Kommunalautonomie, die den Gemeinden das Recht zugesteht, Aufgaben in Selbstverwaltung und -verantwortung zu übernehmen, für eine Trennung von Staat und Kommunen.

2) Vgl. F. K. Mann, Die intermediären Finanzgewalten und ihr Einfluß auf Deutschlands finanzielle Belastung, Jb.Nat.Stat. Bd. 129 (1928), S. $219 \mathrm{ff}$, insbesondere S. 224; vgl. auch derselbe, Die Staatswirtschaft in unserer Zeit, Jena 1930, S. $18 \mathrm{ff}$.

3) Einen überblick über die traditionellen Merkmale parafiskalischer Gejilde gibt K. Tiepelmann, Parafiski, in: Wirtschaftsstudium, 4. Jg. (1975), S. $29 \overline{\mathrm{ff} \text {. }}$ 
Übersicht 5: Die Institution der Parafiski

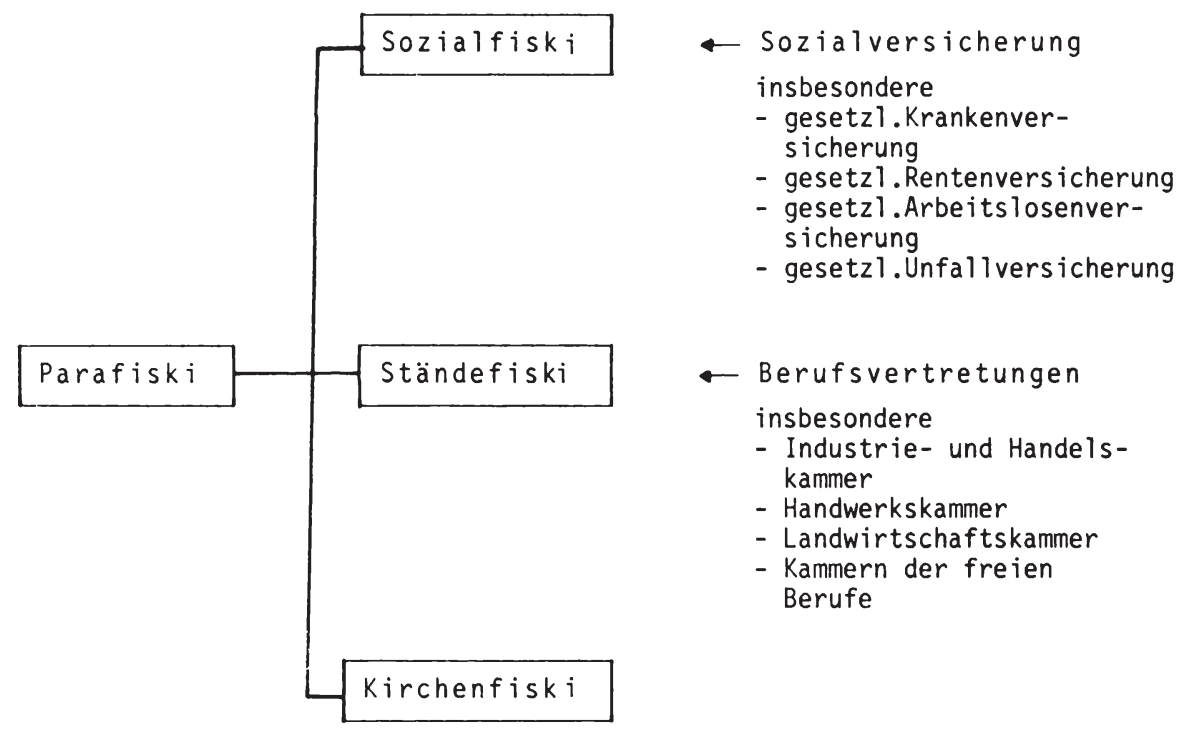

sten Merkmal eine klare Abgrenzung zu den ubrigen nichtstaatlichen Institutionen gewonnen und mit $\mathrm{Hilfe}$ des zweiten Merkmals eine eindeutige Trennung gegenüber den Gebietskörperschaften möglich. Strittig bleibt aber die Einordnung der Kirchenfiski. 1) Obwohl die Kirchen nicht mit dem Recht der Erhebung von Zwangsbeiträgen ausgestattet sind, ist aufgrund der Einziehung der Kirchensteuer durch die Finanzämter und aufgrund der Rechtsstellung der Kirche als öffentlich-rechtliche Körperschaft ${ }^{2}$ ) eine Zuordnung zu den Parafiski gerechtfertigt. Die Charakterisierung der so abgegrenzten parafiskalischen Institu-

1) Vgl. G. Schmölders, Finanzpolitik, 3. Aufl., Berlin u.a., S. 39 sowie H. Zimmermann, K.-D. Henke, Finanzwissenschaft, München 1975, S. 23.

2) Zu der mit dieser Rechtsstellung verbundenen Problematik vgl. Th. Maunz, Der öffentliche Charakter der kirchlichen Aufgaben, in: Festschrift für E. Forsthoff zum 70. Geburtstag, hrsg. von R. Schnur, 2. Auf1., München 1974 , S. $229 \mathrm{ff}$. 
tionen als öffentliche Träger bereitet demgegenüber keine große Mühe. Sowohl die Eigentumsverhältnisse als auch ihr Auftreten in der Rechtsform einer öffentlich-rechtlichen Körperschaft ermöglichen eine zweifelsfreie Identifikation der Finanzintermediäre als öffentliche Aufgabenträger.

Die von einigen Autoren praktizierte extensive Ausweitung des Begriffes Parafiskus auf mehr oder weniger alle intermediären Gruppen $z$ wischen Stat und privater Wirtschaft ${ }^{1}$ ) scheint dagegen unzweckmäßig, gehen doch dadurch gerade die oben herausgearbeiteten besonderen Eigenschaften des Parafiskus wieder verloren. 2)

\subsection{Beliehene}

Nimmt im Regelfall der Staat hoheitliche Funktionen durch seine Behörden bzw. durch verselbständigte Verwaltungsträger wahr oder erklärt er einige öffentliche Träger zu Hilfsfisken, so lassen sich darüber hinaus auch Fälle finden, in denen Private mit Hoheitsbefugnissen ausgestattet sind. Diese Privaten figurieren dann unter dem Begriff "Beliehene". Während die Wirtschaftswissenschaft zum Komplex des Beliehenen bisher keine Aussagen getroffen hat, besitzt die Auseinandersetzung mit dieser Institution vor allem in der rechtswissenschaftlichen Literatur eine lange Tradition ${ }^{3)}$, ohne daß allerdings bis heute eine klare Verständigung über Wesen und Funktion der Beleihung erzielt worden wäre. ${ }^{4)}$ Einen Grund für die starke Beachtung, die das Institut

1) Vgl, etwa Ch. Smekal, Die Finanzwirtschaft intermediärer Gruppen, Innsbruck 1969, S. $50 \mathrm{ff}$; D. Lepelmeier, Soziale Sicherung und Parafiskalität, Frankfurt/M. 1979, \$. 75 ff.

2) Wenig gewonnen scheint auch mit Versuchen, die Zugehörigkeit bestimmter Institutionen zu den Parafiski zu konstruieren. So wird z.B. auch die Unterstellung, die Mitgliedschaft in einer Gewerkschaft beruhe teilweise auf offenem oder verstecktem Zwang, als Argument für eine Zuordnung der Gewerkschaften zu den Parafiski herangezogen, vgl. dazu G. Schmölders, Finanzpolitik, a.a.0., S. 39.

3) Einen Überblick über das umfangreiche Schrifttum gibt F. Ossenbühl, a.a.0.,
S. $138 \mathrm{f}$.

4) Vgl. dazu etwa R. Michael is, Der Beliehene, Diss., Münster 1969, S. $6 \mathrm{ff}$. sowie E. V. Heyen, Das staatstheoretische und rechtstheoretische Problem des Beliehenen, Berlin 1973. 
der Beleihung in dieser Disziplin gefunden hat, sieht OSSENBOHL darin, daß "es als bequemes Sammelbecken für alle jene Phänomene diente, bei denen in irgendeiner Weise private Personen oder Institutionen über den bislang als Privatsphäre verstandenen Raum hinaus in den öffentlichrechtlichen oder auch nur öffentlichen Bereich hineinwirkten."1) Schärfere Konturen erhält der Begriff des Beliehenen dann, wenn darunter alle natürlichen oder juristischen Personen des Privatrechts, also ein Einzelner, eine Gesellschaft oder eine Vereinigung, verstanden werden ${ }^{2)}$, denen der staat die Befugnis zur selbständigen Ausübung bestimmter Kompetenzen der hoheitlichen Verwaltung verliehen hat. ${ }^{3)}$ Als typische Beliehene können danach die Verkehrssicherheitsüberprüfungen vornehmenden Technischen überwachungsvereine, die polizeiliche Gewalt an Bord von Flugzeugen oder Schiffen ausübenden Piloten bzw. Kapitäne sowie der mit der Feuerstättenschau und der Bauabnahme betraute Bezirksschornsteinfegermeister genannt werden. ${ }^{4)}$ Als Ziele der Beleihung werden in erster Linie die Ausnutzung der Initiative und des Sachverstandes der Privaten für die öffentliche Verwaltung sowie die Inanspruchnahme privater situationsbeherrschung genannt. ${ }^{5)}$ Die ökonomische Zweckmäßigkeit einer solchen Konstruktion wird besonders im Hinblick auf die Obertragung spezieller Befugnisse auf Flug- und Schiffskapitäne deutlich. Im allgemeinen dürften die Kapitäne die polizeiliche Gewalt an Bord hinreichend effektiv ausüben. Hingegen widerspräche es allen Effizienzüberlegungen, falls mitreisende Polizisten diese Aufgabe wahrnehmen würden.

1) F. Ossenbuihl, a.a.0., S. 142.

2) In der älteren Literatur werden auch juristische Personen des öffentlichen Rechts zu den Beliehenen gezählt; vgl. dazu U. Steiner, offentliche Verwaltung durch Private, Hamburg 1975, S. 204.

3) Vgl. H. J. Wolff, 0. Bachof, Verwaltungsrecht II, 4. Auf1., München 1976, S. 452.

4) Weitere Beispiele bei R. Michaelis, a.a.0., S. $90 \mathrm{ff}$. sowie H. J. Wolff, 0 . Bachof, Verwaltungsrecht II, a.a.0., S. $452 \mathrm{ff}$.

5) So E. R. Huber, a.a.0., S. 534 und F. Ossenbühl, a.a.0., S. 148. 
Während die Zuordnung der Beliehenen zum privaten Sektor anhand der Eigentumsverhältnisse keine Probleme bereitet, ist ihre Rechtsstellung nicht unumstritten. Als natürliche oder juristische Personen des Privatrechts können sie zwar ebenfalls als Aufgabenträger des privaten Sektors eindeutig identifiziert werden, aufgrund der ihnen verliehenen hoheitlichen Funktionen werden sie jedoch gleichzeitig als Träger von Verwaltungskompetenzen organisationsrechtlich an die Seite der juristischen Personen des öffentlichen Rechts gestellt. 1) "Diese Verquickung privatrechtlicher und öffentlich-rechtlicher Elemente charakterisiert den einzigartigen Status des Beliehenen." 2 ) MICHAELIS sieht in der besonderen Stellung der Beliehenen sogar eine organisatorische Rechtsstellung sui generis, die sich aus deren rechtich selbständigen und in eigenem Namen vorgenommenen Ausubung spezieller staatlicher Verwaltungsfunktionen ergibt, d.h. aufgrund der Wahrnehmung von Rechten und Pflichten für den Stat, ohne daß dieses Tätigwerden der Beliehenen dem Staat zugerechnet wird. ${ }^{3)}$ Dieser Auffassung soll hier allerdings nicht gefolgt, sondern vielmehr das Auftreten des Beliehenen als Subjekt des Privatrechts als hinreichende Bedingung für seine Zuordnung zum privaten sektor angesehen werden.

1) Vgl. U. Steiner, a.a.0., S. 222 sowie H. J. Wolff, 0. Bachof, Verwaltungsrecht II, a.a.0., S. $452 \mathrm{f}$.

2) U. Steiner, a.a.0., S. 225.

3) Vgl. R. Michaelis, a.a.0., S. 129. 


\subsection{Unternehmen}

Als einer systematischen Erörterung nur schwer zugänglich erweist sich das äußerst heterogene Gebilde der Unternehmen. 1) Die amtliche Statistik nimmt keine Trennung zwischen öffentlichen und privaten Unternehmen vor, sondern regelt die Grenzziehung durch Konvention. Sowohl in den Volkswirtschaftlichen Gesamtrechnungen als auch in der Finanzstatistik werden alle Unternehmen unabhängig von ihren Eigentumsverhältnissen und ihrer Rechtsform dem privaten Sektor zugeordnet. Eine Unterscheidung wird lediglich zwischen Unternehmen und öffentlichen Einrichtungen getroffen. Letztere werden mit Hilfe der Enumeration bestimmt und dem öffentlichen Sektor, genauer: den Einrichtungen der Gebietskörperschaften, zugewiesen. So gehören insbesondere die Einrichtungen der Sozial-und Jugendhilfe, z.B. Altenheime, Erziehungs - und Jugendbildungsheime, ferner Einrichtungen des Gesundheitswesens sowie sonstige Einrichtungen wie z.B. Viehund Schlachthöfe, Feuerwehr und Bestattungseinrichtungen zum öffentlichen Sektor, während u.a. kommunale Versorgungs- und Verkehrsunternehmen zum privatwirtschaftlichen Unternehmenssektor gezählt werden. 2) Auch wenn diese Aufzählung kein klares Zuordnungskriterium erkennen 1 äßt, so wurde als Grundgedanke der $A b-$ grenzung betont, daß alle jene Institutionen als dem öffent1 ichen Sektor zugehörig betrachtet werden können, die staat1 iche Aufgaben durchführen sowie Güter und Dienstleistungen

1) Eine sachliche Differenzierung zwischen den Begriffen "Unternehmung" und "Betrieb", wie sie z.B. Rieger bereits 1928 vornahm, indem er den Betrieb als "Vehikel der Unternehmung" bezeichnete, ist im Zusammenhang mit einer Abgrenzung öffentlicher lind privater Aufgabenträger ohne Bedeutung; vgl. W. Rieger, Einfuihrung in die Privatwirtschaftslehre, 3. Aufl., Erlangen 1964, S. 40; im überblick dazu auch G. Wöhe, Einführung in die allgemeine Betriebswirtschaftslehre, 12. Auf1., Múnchen 1976, S. 2 ff. Unerheblich ist deshalb auch, ob als Oberbegriff der Terminus "öffentliche Betriebe" oder "öffentliche Unternehmen" zu verwenden ist. Von öffentlichen Betrieben sprechen u.a. Th. Thiemeyer, Wirtschaftslehre öffentlicher Betriebe, Reinbek bei Hamburg 1975; A. Schnettler, Offentliche Betriebe, Essen 1956.

2) Vgl. G. Hamer, Revidierte Konten der Volkswirtschaftlichen Gesantrechnungen, WiSta 1970, S. 283. 
unter Verzicht auf regelmäßige Gewinnerzielung bereitstellen. 1) "Man hat jedoch in der Praxis nicht immer allein auf das Grundprinzip abstellen können; kasuistische Entscheidungen waren unumgänglich."2)

Mit dem Hinweis auf die Zielsetzung dominieren in der amt ichen Statistik also funktionale Gesichtspunkte. Damit rückt sie in die Nähe jener Versuche, die eine Abgrenzung öffentlicher Unternehmen anhand des konstruierten Gegensatzpaares "Gemeinwirtschaft" und "Privatwirtschaft" vornehmen, d.h. losgelöst von der Frage des Trägers wird "Gemeinwirtschaft" zum Sammelbegriff für alle nicht privatwirtschaftlich orientierten wirtschaftseinheiten. Die Auseinandersetzung um diese beiden Begriffe zeigt jedoch, daB eine funktionale Abgrenzung zu keinem eindeutigen Ergebnis fuihrt.

Wird auf eine inhaltiche Konkretisierung der "Gemeinwirtschaftlichkeit" verzichtet und statt dessen die Ausgestaltung der Eigentumsverhältnisse zum ausschlaggebenden Abgrenzungskr.iterium erhoben, so können folgende gemeinwirtschaftliche Unternehmenstypen unterschieden werden: ${ }^{3)}$

- Offentliche Unternehmen, die vollständig im Eigentum der öffentlichen Hand stehen;

- freigemeinwirtschaftliche Unternehmen, die von - in diesem Sinne - "freien" gesellschaftlichen Gruppen wie z.B. Gewerkschaften betrieben werden;

- öffentlich gebundene Unternehmen, die ausschließlich in privatem Eigentum geführt werden und privatwirtschaftlichen Zielsetzungen folgen, jedoch Sondervorschriften unterworfen

1) So H. Bartels, Das Einkommenskonto für den Staat in den Volkswirtschaft1 ichen Gesamtrechnungen, WiSta 7. Jg. (1955), S. 119.

2) G. Bombach, Die öffentliche Finanzwirtschaft im Wirtschaftskreislauf, HdF Bd. 1, 3. Auf1., Tübingen 1978, S. 59.

3) Vgl. G. Rittig, Die Definitionen des Terminologie-Ausschusses der Gesel1schaft zur Förderung der öffentlichen Wirtschaft, AöfU Bd. 1 (1954), S. 222 . 
sind, die den Spielraum ihrer wirtschaftlichen Betätigung einschränken. 1)

Das institutionelle Kriterium Eigentumsverhältnisse dient nicht nur einer Untergliederung der gemeinwirtschaftlichen Unternehmen, sondern kann auch zur Abgrenzung öffentlicher und privater Betriebe herangezogen werden. Neben den im statlichen Eigentum befindlichen öffentlichen Unternehmen sind dann die gemischtwirtschaftlichen Unternehmen, in denen öffentliche und private Anteilseigner zusammenwirken, sowie die von privaten Eigentiumern gefühten privatwirtschaftlichen Unternehmen zu unterscheiden. Eine solche Abgrenzung ist allerdings immer nur dann ohne Schwierigkeiten durchführbar, wenn in den öffentlichen Unternehmen ausschließlich öffentliche und in den privaten Unternehmen lediglich private Anteilseigner zu finden sind. Sobald irgendein "Mischverhältnis" der Anteile festzustellen ist, muß entschieden werden, ob bereits von einem gemischtwirtschaftiichen Unternehmen gesprochen werden kann oder ob die Dominanz des öffentlichen bzw. privaten Eigentums die Zuordnung zu einer der beiden "reinen" Formen zweckmäßig erscheinen läßt. 2) Wann ein Unternehmen als gemischtwirtschaftlich zu gelten hat, ist strittig. Wird als Entscheidungskriterium auf die Höhe der Beteiligung am Grund-oder Stammkapital abgestellt, so wird überwiegend eine Beteiligung in Höhe der Sperrminorität auf beiden

1) Gegenstand der öffentlichen Bindung können u.a. Preise, Investitionen, Betriebs- und Vertragsbedingungen der Unternehmen sein (z.B. Anschlußpflicht, Betriebspflicht, Fahrplanpflicht, Tarifpflicht). Öffentlich gebunden sind in der Bundesrepublik insbesondere private Unternehmen der Elektrizitäts-, Gas- und Wasserwirtschaft, Kredit- sowie Verkehrsunternehmen. Ausführlich hierzu G. von Eynern, Das öffentlich gebundene Unternehmen, AöfU Bd. 4 (1958), S. $1 \mathrm{ff} . ; \mathrm{vgT}$. auch H. J. Kleinsteuber, Art. offentliche Bindung, in: Wörterbuch zur politischen Okonomie, 2. Aufl., Opladen 1977, S. $299 \mathrm{ff}$.

2) Dieter Bös bezeichnet beispielsweise ein Unternehmen bereits dann als öffentlich, wenn es sich teilweise im Eigentum der öffentlichen Hand befindet; vgl. D. Bös, offentliche Unternehmen, HdF 2. Bd., 3. Auf1., Tübingen 1980, S. 4. Theo Thiemeyer verlangt für den gleichen Sachverhalt dagegen immerhin einen Träger, der "zum überwiegenden Teil die öffentliche Hand ist"; Th. Thiemeyer, a.a.0., S. 19. 
Seiten als Voraussetzung gefordert ${ }^{1}$ ), daneben werden aber auch abweichende Prozentzahlen genannt. 2) Einige Autoren betonen darüber hinaus, daß als begriffsnotwendiges Merkmal gemischtwirtschaftlicher Unternehmen nicht lediglich das Verhältnis der kapitalmäßigen Beteiligung, sondern vor allem der davon möglicherweise abweichende effektive Einfluß der öffentlichen bzw. privaten Träger auf das Unternehmen von vorrangiger Bedeutung sei. 3) VON LOESCH spricht sich deshalb sogar gegen eine isolierte Betrachtung der gemischtwirtschaftlichen Unternehmen aus, da je nachdem, welcher der Träger dominiere und seine Zielvorstellungen durchsetzen könne, eine Zuordnung zu den öffentlichen bzw. privatwirtschaftichen Unternehmen zu erfolgen habe. ${ }^{4)}$

Alternativ kann eine Unterscheidung der Unternehmenstypen anhand der Rechtsform vorgenommen werden. Das Spektrum der Rechtsformen, in denen Unternehmen auftreten können, ist dabei äußerst breit. Es reicht von den Betrieben des Bundes und der Länder, die nach den Vorschriften des Regiebetriebsrechts gefüht werden und den kommunalen Betrieben, die dem Eigenbetriebsrecht unterliegen, über Unternehmen in der öffentlichrechtlichen Form einer Körperschaft, Anstalt oder Stiftung bis hin zu den privatrechtlich firmierenden Eigengesellschaften in der Rechtsform der $A G$ und $G m b H$ und den als KG oder OHG geführten Personengesellschaften. 5 )

1) Vgl. u.a. P. van Aubel, Die gemischtwirtschaftliche Unternehmung, in: Handbuch der Kommunaten Wissenschaft und Praxis, 3. Bd., Berlin u.a. 1959, S. 877; A. Schnettler, a.a.0., S. 34.

2) So z.B. V. Emmerich, Das Wirtschaftsrecht der öffentlichen Unternehmen, Bad Homburg v.d.H. 1969, S. 59.

3) $\mathrm{Vgl}$. P. Eichhorn, Zum Begriff der gemischtwirtschaftlichen Unternehmung, BFuP 18. Jg. (1966), S. $616 \mathrm{ff}$.; so auch bereits E. Schmelcher, Art. Gemischtwirtschaftliche Unternehmen, HdSt 4. Bd., Jena 1927, S. 846.

4) Vgl. A. von Loesch, Die gemischtwirtschaftliche Unternehmung, Köln 1977 , S. 111 .

5) Vgl. dazu ausführlich P. Eichhorn, Struktur und Systematik kommunaler Betriebe, Stuttgart 1969 , S. $50 \mathrm{ff}$; H. H. Klein, Die Teilnahme des Staates am wirtschaftlichen Wettbewerb, Stuttgart 1968, S. 33 ff.; G. Puittner, Die öffentlichen Unternehmen, Bad Homburg v.d.H. 1969, S. $98 \mathrm{ff} . ;$ Th. Thiemeyer, a.a.0., S. $34 \mathrm{ff}$. 
Obersicht 6: Unternehmenstypologie anhand der Unterscheidungsmerkmale Eigentums -

verhältnisse und Rechtsform

\begin{tabular}{|c|c|c|c|}
\hline $\begin{array}{l}\text { Abgrenzungs- } \\
\text { kriterium } \\
\text { Unterneh- } \\
\text { menstyp }\end{array}$ & $\begin{array}{l}\text { Eigentums- } \\
\text { verhältnisse }\end{array}$ & Rechtsform & Beispiele \\
\hline $\begin{array}{l}\text { öffentliche } \\
\text { Unternehmen }\end{array}$ & staatlich & $\begin{array}{l}\text { öffentlich-rechtlich } \\
\text { und privatrechtlich } \\
\text { - Regiebetrieb } \\
\text { - Eigenbetrieb } \\
\text { - Körperschaft, } \\
\text { Anstalt, Stiftung } \\
\text { - Kapitalgesellschaft }\end{array}$ & $\begin{array}{l}\text { - Stadtwerke Bonn } \\
\text { - Hamburger Wasser- } \\
\text { werke }\end{array}$ \\
\hline $\begin{array}{l}\text { gemischtwirt- } \\
\text { schaftl iche } \\
\text { Unternehmen }\end{array}$ & $\begin{array}{c}\text { staatlich } \\
\text { und } \\
\text { privat }\end{array}$ & $\begin{array}{l}\text { privatrechtlich } \\
\text { - Kapitalgesell- } \\
\text { schaft }\end{array}$ & $\begin{array}{l}\text { - VEBA } \\
\text { - Volkswagenwerk } \\
\text { - Preussag }\end{array}$ \\
\hline $\begin{array}{l}\text { freigemein- } \\
\text { wirtschaftliche } \\
\text { Unternehmen }\end{array}$ & privat & $\begin{array}{l}\text { privatrechtlich } \\
\text { - Kapitalgesell- } \\
\text { schaft } \\
\text { - Personengesell- } \\
\text { schaft }\end{array}$ & $\begin{array}{l}\text { - Bank für Gemein- } \\
\text { schaft } \\
\text { - Neue Heimat } \\
\text { - Volksfürsorge }\end{array}$ \\
\hline $\begin{array}{l}\text { öffentlich } \\
\text { gebundene } \\
\text { Unternehmen }\end{array}$ & privat & $\begin{array}{l}\text { privatrechtlich } \\
\text { - Kapitalgesel1- } \\
\text { schaft } \\
\text { - Personengese11- } \\
\text { schaft }\end{array}$ & $\begin{array}{l}\text { - Banken } \\
\text { - Versicherungs- } \\
\text { unternehmen } \\
\text { - Verkehrsunter- } \\
\text { nehmen }\end{array}$ \\
\hline $\begin{array}{l}\text { private } \\
\text { Unternehmen }\end{array}$ & privat & $\begin{array}{l}\text { privatrechtlich } \\
\text { - Kapitalgesell- } \\
\text { schaft } \\
\text { - Personengesell- } \\
\text { schaft }\end{array}$ & $\begin{array}{l}\text { - BASF } \\
\text { - BMW } \\
\text { - Mannesmann }\end{array}$ \\
\hline
\end{tabular}


In der Ubersicht 6 sind die unterscheidbaren Unternehmenstypen und die diskutierten Differenzierungsmöglichkeiten überblickartig dargestellt. Wird allein die Rechtsform zum Unterscheidungskriterium erhoben, so beschränken sich die dem öffentlichen Sektor zugehörigen Wirtschaftseinheiten auf jenen Teil der öffentlichen Unternehmen, die als Regiebetrieb, Eigenbetrieb, Körperschaft, Anstalt oder Stiftung im öffentlich-rechtlichen Rahmen agieren. Der Bereich der als öffentliche Aufgabenträger eingestuften Unternehmen fällt hingegen etwas größer aus, falls das Kriterium Eigentumsverhältnisse zugrundegelegt wird. Nunmehr gehören nicht nur alle öffentlichen Unternehmen zum öffentlichen sektor, sondern darüber hinaus zumindest auch diejenigen gemischtwirtschaftlichen Unternehmen, deren Kapitalanteile sich überwiegend im Eigentum der öffentlichen Hand befinden.

\subsection{Freie Berufe}

Im Zuge der anhaltenden Privatisierungsdebatte und der damit einhergehenden Fijlle von Vorschlägen zur Obertragung öffentlicher Aufgaben auf den privaten Sektor sind auch die freien $\mathrm{Be}-$ rufe auf besonderes Interesse gestoßen. 1) Wie die privatwirtschaftlichen Unternehmen erbringen sie Güter und Dienstleistungen gegen spezielles Entgelt, welches in der Regel eine Gewinnerzielung ermöglicht, zumindest aber die Deckung der Kosten erlaubt. In den Volkswirtschaftlichen Gesamtrechnungen wird deshalb keine Trennung zwischen freien Berufen und sonstigen Unternehmen, sondern eine gemeinsame Subsumtion unter dem Unternehmenssektor vorgenommen. 2 )

Allerdings werden den freien Berufen besondere wirtschaftliche und gesellschaftspolitische Funktionen zugewiesen und sie explizit mit der Erfüllung öffentlicher Aufgaben in Zusammenhang

1) Vgl. dazu insbesondere Studiengruppe für Sozialforschung e.V., a.a.0.

2) Vgl. H. Bartels, Das Kontensystem für die Volkswirtschaftlichen Gesamtrechnungen der Bundesrepublik Deutschland, WiSta 1960, S. 321 f. 
gebracht. 1) Außerdem wird eine "häufig dem Gemeinwohl verpflichtete Berufsausuibung" 2 ) konstatiert, und es wird ihnen sogar eine Mission aufgetragen: "Als Mittler und Makler zu mitmenschlicher Einheit, als 'Statthalter der Freiheit' im Rechtsstaat, als bewahrende, wählerische und schöpferische Elemente in der Kulturgesellschaft sind sie unverzichtbar, wenn dieser Staat, wenn diese Gesellschaft eine kulturelle und soziale Heimat bleiben oder werden sollen, in denen es sich lohnt zu leben."3)

Bei nuichterner Betrachtung stellt sich allerdings das Anliegen der freien Berufe als weit weniger anspruchsvoll dar. Als zentrale Merkmale der freiberuflichen Tätigkeit werden genannt: ${ }^{4}$ )

- wirtschaftlich selbständige Ausübung der Berufstätigkeit;

- Angebot "geistiger", d.h. im allgemeinen auf qualifizierter Ausbildung beruhender Leistungen;

- Erbringung persönlicher Leistungen, wobei auch in eigener Leitung und Verantwortung des freiberuflich Tätigen gestaltete personale oder materielle Fremdleistungen einbezogen werden können.

Die freiberufliche Tätigkeit zeichnet demnach einerseits die eigene Risikotragung und andererseits die öffentliche Bindung aus, die sich daraus ergibt, daB Art der Leistungserstellung sowie Preisgestaltung ( $z$. B. Gebührenordnungen, Festspannenregelungen) staatlichen Regelungen unterliegen. Aus diesem Sachverhalt läßt sich jedoch kaum eine Aussage darüber treffen, ob es sich bei der Tätigkeit freier Berufe generell um die Wahrnehmung öffentlicher Aufgaben handelt bzw. inwieweit die öffentliche Aufgabenerfüllung über eine Gewinnerzielungs-

1) Vgl. J.F. V. Deneke, Die freien Berufe, Stuttgart 1956, S. 319.

2) Bericht der Bundesregierung über die Lage der freien Berufe in der BundesrepubTik Deutschland, Bundestagsdrucksache 8/3139 vom 29.8.1979, S. 39 .

3) So das prätentiöse Resümee von J.F. V. Deneke, Die freien Berufe, a.a.0., S. 347 .

4) Vgl. den Bericht der Bundesregierung, a.a.0., S. 7. 
absicht dominiert. Zwar stellt die Rechtsprechung im Hinblick auf die Apotheker ausdrücklich fest, daß diese zur Erfüllung öffentlicher Aufgaben tätig werden und deshalb bei ihrer $\mathrm{Be}-$ rufsausuibung das Gewinnstreben in den Hintergrund zu treten hat. 1) Gleichwohl düfte nur schwer zu klären sein, ob sich freie Berufe in ihrer Zielsetzung grundsätzlich von den privatwirtschaftlichen Unternehmen unterscheiden. Bei letzteren ist die Gewinnmaximierung als vorrangiges Ziel allgemein anerkannt. Ob der Apotheker, der Wirtschaftsprüfer, der Dolmetscher oder Musiker überwiegend finanzielle Interessen entwickelt oder sich in erster Linie der Erfüllung öffentlicher Aufgaben verpflichtet fühlt, muß aber offen bleiben.

\subsection{Verbände}

Seit Jahren stehen die Verbände vor allem deshalb im Blickpunkt des Interesses und namentlich der Kritik, weil sie erheblichen politischen Einfluß ausüben. Beklagt wird die "Herrschaft der Verbände"2), und Schlagworte wie "Lobbyismus", "Pressure Groups", "Gewerkschaftsstaat" und "Aushöhlung der Demokratie" durch Interessengruppen machen die Runde. 3 ) Während in der öffentlichen Diskussion insbesondere dieser soziologisch-politische Aspekt des Verbandswesens hervorgehoben wird, indem Verbände als organisationen bzw. Zusammenschlüsse begriffen werden, "denen es um die Anmeldung und Durchsetzung politischer Interessen im politischen Willensbildungsprozeß geht" 4 ), ist aus ökonomischer Sicht von besonderem Interesse, $d a B$ ein großer Teil der Verbände nicht primär eine politische Einflußnahme anstrebt, sondern in Form der Bereitstellung von Gütern und Dienstleistungen bei der Erfuillung öffentlicher Aufgaben mitwirkt. ${ }^{5}$ ) Zu nennen sind vor

1) Vgl. BVerfGE 17, 239.

2) Th. Eschenburg, Herrschaft der Verbände?, Stuttgart 1956.

3) Vgl. E. Tuchtfeldt, Wirtschaftspolitik und Verbände, in: Hamburger Jahrbuch für Wirtschafts- und Gesell schaftspolitik, 2. Jg. (1956), S. 72.

4) D. Obendörfer, Zur Typologie der Verbände, in: Staatsführung, Verbandsmacht und innere Souveränität, hrsg. von K. H. Biedenkopf und R. von Voss, Stuttgart 1977, S. 20.

5) $\mathrm{Vgl}$. dazu insbesondere W. Kirberger, a.a.0. 
allem die zahlreichen als Verbände auftretenden privaten Träger im Bereich des Gesundheits- und Sozialwesens wie z.B. das Deutsche Rote Kreuz, die Caritas oder der Deutsche Paritätische Wohlfahrtsverband. ") Die Vielfalt der Verbände kommt auch darin zum Ausdruck, daß sie unter so unterschiedlichen Bezeichnungen wie Ausschuß, Bund, Gemeinschaft, Gesellschaft, Institut, Kammer, Kreis, Kuratorium, Rat, Ring, Tag, Verband, Verein oder Zentrale auftreten. 2)

Ein Blick auf die Rechtsformen der Verbände zeigt, daß die Mehrzahl der Verbände privatrechtlich als rechtsfähiger Verein organisiert ist. Eine Ausnahme bilden die Gewerkschaften und Arbeitgeberverbände, die als nicht rechtsfähige Vereine fungieren. Daneben kommen auch Gesellschaften ( $A G$, GmbH) und Personengesellschaften in Frage und Verbände, die als Körperschaften des öffentlichen Rechts verfaßt sind, denn "je föderalistischer ein stat aufgebaut ist, je selbständiger die einzelnen regionalen und fachlichen Gliederungen des Statsverbandes sind, desto offener treten öffentliche Körperschaften selbst als Verbände in Erscheinung. 3) Erwähnt sei hier lediglich der Deutsche Städtetag als Verband der kreisfreien Städte oder die Kommunale Gemeinschaftsstelle für Verwaltungsvereinfachung (KGSt). Da ihre Mitglieder aber mit Hoheitsrechten

1) Eine scharfe Trennung zwischen Verbänden mit bloßem Forderungscharakter und solchen, die ausschließlich Güter und Dienstleistungen anbieten, dürfte kaum möglich sein. So vertreten z.B. Gewerkschaften nicht nur die Interessen der Arbeitnehmer in Tarifverhandlungen oder durch ihre Forderung nach Mitbestimmung, sondern sie werden daneben auch leistend tätig, wenn sie Seminare für berufliche oder außerberufliche Bildung anbieten. Das gleiche gilt für den ADAC, der nicht nur im Namen seiner Mitglieder die Forderung nach einer allgemeinen Anschnallpflicht erhebt oder sich gegen Geschwindigkeitsbegrenzungen ausspricht, sondern gleichzeitig auch Leistungen wie z.B. Pannenhilfe auf Autobahnen erbringt. Deshalb ist eine Klassifizierung, die eine grobe Zweiteilung der Verbandsinteressen in soziale bzw. ideelle und ökonomische bzw. materielle vornimmt, wenig hilfreich; vgl. dazu K. von Beyme, Interessengruppen in der Demokratie, 4. Auf1., Muinchen 1974, S. 32.

2) Vgl. R. Breitling, Die Verbände in der Bundesrepublik, Meisenheim am Glan 1955, S. 5 f.

3) Ebenda, S. 64. 
ausgestattete Gebietskörperschafter sind, sollen diese hier nicht den Verbänden zugeordnet werden. Schließlich wird eine beträchtliche Anzahl der Verbände in der Organisationsform des halbstaatlichen Vereins geführt. Halbstatliche Vereine zeichnen sich durch ein finanzielles und personelles Zusammenwirken privater und staatlicher Träger aus. 1)

\subsection{Selbstorganisationen}

Unter dem Begriff "Selbstorganisation" soll eine weitere Kategorie der Aufgabenträger zusammengefaßt werden, die in den vergangenen Jahren erheblich an Aufmerksamkeit gewonnen hat. BADELT subsumiert unter diesem Sammelbegriff verschiedene Formen der Bürgerselbsthilfe wie z.B.: ${ }^{2)}$

- ein "Verein" zur Organisation eines Nachbarschaftshilfesystems;

- eine "Bürgerinitiative" zur Errichtung eines Spielplatzes;

- eine "Selbsthilfegruppe" zur Bewältigung des Problems unvollständiger Familien;

- eine "Interessengemeinschaft" von Pendlern zur Aufstellung regelmäßiger Mitfahrgelegenheiten zwischen Wohnung und Arbeitsplatz;

- eine "Kleingenossenschaft" zur Gestaltung einer Parkanlage oder eines Erholungsgebietes.

Ausgegrenzt werden von BADELT dagegen u.a. Bürgerinitiativen mit reinem Protest- und Forderungscharakter, traditionelle Interessensvertretungen (z.B. Gewerkschaften, Arbeitgeberverbände, Wirtschaftsverbände) sowie Großvereine und Non-

1) Vgl. dazu insbesondere F. Muiller-Thoma, Der halbstaatliche Verein, Berl in 1974. Während W. Kirberger, a.a.0., S. $104 \mathrm{f}$. trotz des Zusammenwirkens privater und staatTicher Kräfte eine den "privaten Verbänden" gleichgestellte Behandlung des halbstaatlichen Vereins vornimmt, grenzt Schmölders diesen aus der Betrachtung der Verbände aus; vg1. G. Schmölders, Das Selbstbildnis der Verbände, SchdVSocpol N.F., Bd. 38 , Berlin 1965, S. 13.

2) Vgl. Ch. Badelt, Sozioökonomie der Selbstorganisation, a.a.0., S. 30. 
Profit-Organisationen, die den Charakter privater Bürokratien angenommen haben. 1 )

Zwischen der Selbstorganisation und dem Verbandswesen ergeben sich einige Unterschiede in tendenzieller aber auch in grundsätzlicher Hinsicht. überwiegend zeichnet sich die Selbstorganisation durch den Zusammenschluß von Bürgern zu einer eher kleinen und autonom agierenden Gruppe aus, die, im Gegensatz zu den auf Dauer angelegten Verbänden, sich häufig spontan aus einem einzigen konkreten Anlaß zusammenfindet, um sich nach Erreichung des Organisationszieles (z.B. Errichtung eines spielplatzes) wieder aufzulösen. "Selbstorganisation entsteht immer als Reaktion auf das Auftreten eines Problems." 2) Dieses Problem kann einmal ein rein materielles sein. Die Versorgung der privaten Haushalte mit Gijtern und Dienstleistungen weist zum Teil Lïcken auf, die weder von der Privatwirtschaft noch von den öffentlichen Haushalten oder den traditionellen intermediären Gruppen wie Verbände geschlossen werden. Hier werden dann Selbstorganisationen aktiv, um diese versorgungsmängel zu beseitigen. Selbstorganisationen können aber auch das Resultat eines eher immateriellen Problems sein. Individuen schließen sich dann nicht mehr nur dort zusammen, wo es Versorgungsiucken zu beheben gilt, sondern die Selbstorganisation erhält einen Eigenwert, d.h. die Mitglieder der Gruppe ziehen aus der Art der Bereitstellung dieser Guiter und Dienstleistungen, die auch von privaten Unternehmen bzw. öffentlichen Institutionen angeboten werden, einen Nutzen, der über den rein materiellen Nutzen der Güter hinausgeht. Selbstorganisation wird hier mit "Lebensqualität" verbunden und zum Ausdruck einer neuen Form der Emanzipation, eines "alternativen Lebensstils". 3 )

1) Vgl. Ch. Badelt, Sozioökonomie der Selbstorganisation, a.a.0., S. 30 sowie R. Bauer, Wohlfahrtsverbände in der Bundesrepublik, Weinheim und $\mathrm{Ba}-$ sel 1978, S. 40.

2) Ch. Badelt, Sozioökonomie der Selbstorganisation, a.a.0., S. 96.

3) Vgl. dazu auch Ch. Badelt, Selbstorganisation kommunaler Aufaaben, in: Das öfentliche Haushaltswesen in Österreich, 21. Jg. (1980), S. $57 \mathrm{ff}$. 


\subsection{Private Haushalte}

In der Wirtschaftswissenschaft sind die privaten Haushalte üblicherweise in zweifacher Hinsicht Gegenstand der Analyse. Zum einen fragen sie als Konsumenten die von Unternehmen am Markt angebotenen Güter und Dienstleistungen nach und nehmen die vom Staat bereitgestellten öffentlichen Leistungen in Anspruch. Zum anderen bieten sie den Unternehmen und dem Staat Faktorleistungen in Form ihrer Arbeitskraft an. Die den privaten Haushalten zligewiesene Doppelrolle reicht indes nicht aus, die ökonomisch relevante Betätigung dieser Wirtschaftssubjekte vollständig zu erfassen. Zwar werden private Haushalte schon ex definitione nicht in größerem Umfange als Produzenten von am Markt angebotenen Gütern und Diensten tätig, jedoch erbringen auch sie eine Reihe sehr unterschiedlicher Leistungen, die, wïrden sie nicht von den Haushalten bereitgestellt, von anderen Aufgabenträgern übernommen werden müßten. So sind beispielsweise in einigen Gemeinden private Haushalte zur Reinigung der anliegenden Straßen verpflichtet. Per Gesetz überträgt der Stat den Haushalten die Durchführung einer Aufgabe, die er im anderen Falle entweder durch Indienstnahme privater Straßenreinigungsunternehmen oder durch Bedienstete der Gemeinden selbst übernehmen müßte. Von Bedeutung sind aber auch die von den privaten Haushalten freiwillig erbrachten Dienstleistungen, die zu einer Entlastung anderer Aufgabenträger führen. Ebenfalls nur beispielhaft genannt sei hier die Altenpflege im familiären Bereich. War sie früher die Regel, so'stellt sie heute immerhin noch eine Alternative zur staatlichen Altenpflege oder zu den von privaten Verbänden geführten Altenheimen dar. Auch im Rahmen der Nachbarschaftshilfe bietet sich nicht nur durch den Zusammenschluß Privater zu Selbstorganisationen, sondern auch für einzelne Haushalte ein breites Betätigungsfeld (u.a. Babysitting, Krankenpflege, Einkaufshilfe, Reparaturarbeiten, Nachhilfe-Unterricht etc.).

Private Haushalte können somit als eigenständiger Typus eines Aufgabenträgers betrachtet werden. Dabei beschränkt sich die Wahrnehmung von Aufgaben jedoch nicht nur auf die Durchführung 
bestimmter Dienstleistungen, sondern private Haushalte werden auch unterstützend tätig durch finanzielle Hilfsmaßnahmen (z.B. Spende) und haben darüber hinaus in vielen Fällen auch eine regulative Funktion. Letzteres ist jedoch nicht mehr allein mit ökonomischen kategorien zu erfassen. Vorerst mag deshalb hier der Hinweis genügen, daß private Haushalte zumindest in kleinen Gruppen einen sozialen Druck ausüben, der in einem gewissen Umfange unerwünschtes Verhalten (z.B. Drogengenuß, Ehescheidung, asoziales Verhalten) verhindert. Unabhängia davon, ob dieser soziale Druck positiv oder negativ zu bewerten ist, wird durchaus das Eingreifen anderer Aufgabenträger (z. B. Drogenberatunasstelle, durch das staatliche Gesundheitswesen angebotene Entziehungskur) vollends oder teilweise überflüssig.

\section{Die Bedeutung der Trägerschaft als Abgrenzungskriterium für öffentliche und private Aufgaben}

3.1. Die Bedeutung der Rechtsform

Die Typologie der Aufgabenträger hat bereits Hinweise darauf gegeben, daß Wirtschaftssubjekte in einer Vielzahl von Rechtsformen tätig werden können. Auf der einen Seite sind Formen des öffentlichen Rechts wie Behörden, Regie- und Eigenbetriebe, Körperschaften, Anstalten und Stiftungen zu unterscheiden, während auf der anderen Seite Aufgabenträger in ebenso vielfältiger Weise privatrechtlich in der Form einer Eigen-oder Personengesellschaft, als Genossenschaft, private Stiftung oder Verein aktiv werden können.

Eine institutionelle Aufgabenabgrenzung mittels des formalen Kriteriums der Rechtsform beruht nun auf der Vorstellung, daß alle Institutionen, die im öffentlich-rechtlichen Rahmen agieren und damit als öffentliche Träger zu betrachten sind, öffentliche Aufgaben erfuillen; eine Aufgabe dagegen immer dann privaten Charakter besitzt, falls Wirtschaftssubjekte sich in Ausubung ihrer Tätigkeiten einer Form des Privatrechts bedienen. Mit anderen Worten: Die rechtliche Organisationsform der Aufgabenerfüllung wird zum alleinigen Entscheidungskri- 
terium für die Abgrenzung öffentlicher und privater Aufgaben erhoben. In der Übersicht 7 werden die Aufgabenträger den Rechtsformen gegenübergestellt, in denen sie üblicherweise tätig werden. Es zeigt sich, daß eine eindeutige Trennung der Aufgabenträger in öffentlich-rechtliche einerseits und privatrechtliche andererseits nicht möglich ist, sondern eine klare Zuordnung lediglich in wenigen Fällen vorgenommen werden kann. Dies gilt zum einen für die Gebietskörperschaften und den Parafiski, die in der Rechtsform der Behörde bzw. Körperschaft öffentlich-rechtlich tätig werden und damit im Rahmen dieser institutionellen Aufgabenabgrenzung eindeutig als Träger öffentlicher Aufgaben zu identifizieren sind. Demgegenüber können allenfalls die privaten und freigemeinwirtschaftlichen Unternehmen sowie die Zusammenschiusse der Privaten zu Verbänden, Stiftungen oder zur Selbstorganisation ohne Bedenken den privaten Trägern zugerechnet werden, da nur sie ausschließlich privatrechtlich auftreten. Bei Zugrundelegung der rechtlichen organisationsform als Zuordnungskriterium zwischen "öffentlich" und "privat" müßten demnach diese Institutionen ohne Ausnahme private Aufgaben wahrnehmen. Bereits mehrfach ist angeklunaen, daß dieser Schluß voreilig ist.

Insbesondere bezüglich einer Einordnung der Unternehmen erweist sich das formale Kriterium der Rechtsform als äußerst problematisch. Wuirde allein die Rechtsform zum Entscheidungskriterium erhoben, so müßten alle im privatrechtlichen Rahmen auftretenden öffentlichen Unternehmen dem privaten Sektor zugerechnet werden. Daneben würden jedoch z.B. kommunale Eigenbetriebe, die u.U. die gleiche wirtschaftliche Tätigkeit zu den gleichen Konditionen wie ein privatrechtlich agierendes Unternehmen ausüben, nur deshalb dem öffentlichen Sektor zugeordnet, weil sie in öffentlich-rechtlicher Form tätig werden. Die Wahl der Rechtsform als Abgrenzungskriterium muß deshalb insbesondere hinsichtlich einer sachgerechten Zuordnung der Unternehmen auf kommunaler Ebene höchst unbefriedigend bleiben. Da bestimmte Versorgungsleistungen in einigen Gemeinden direkt von den Regie- oder Eigenbetrieben, in anderen Gemeinden dagegen von 
Obersicht 7: Rechtsformen öffentlicher und privater Aufgabenträger

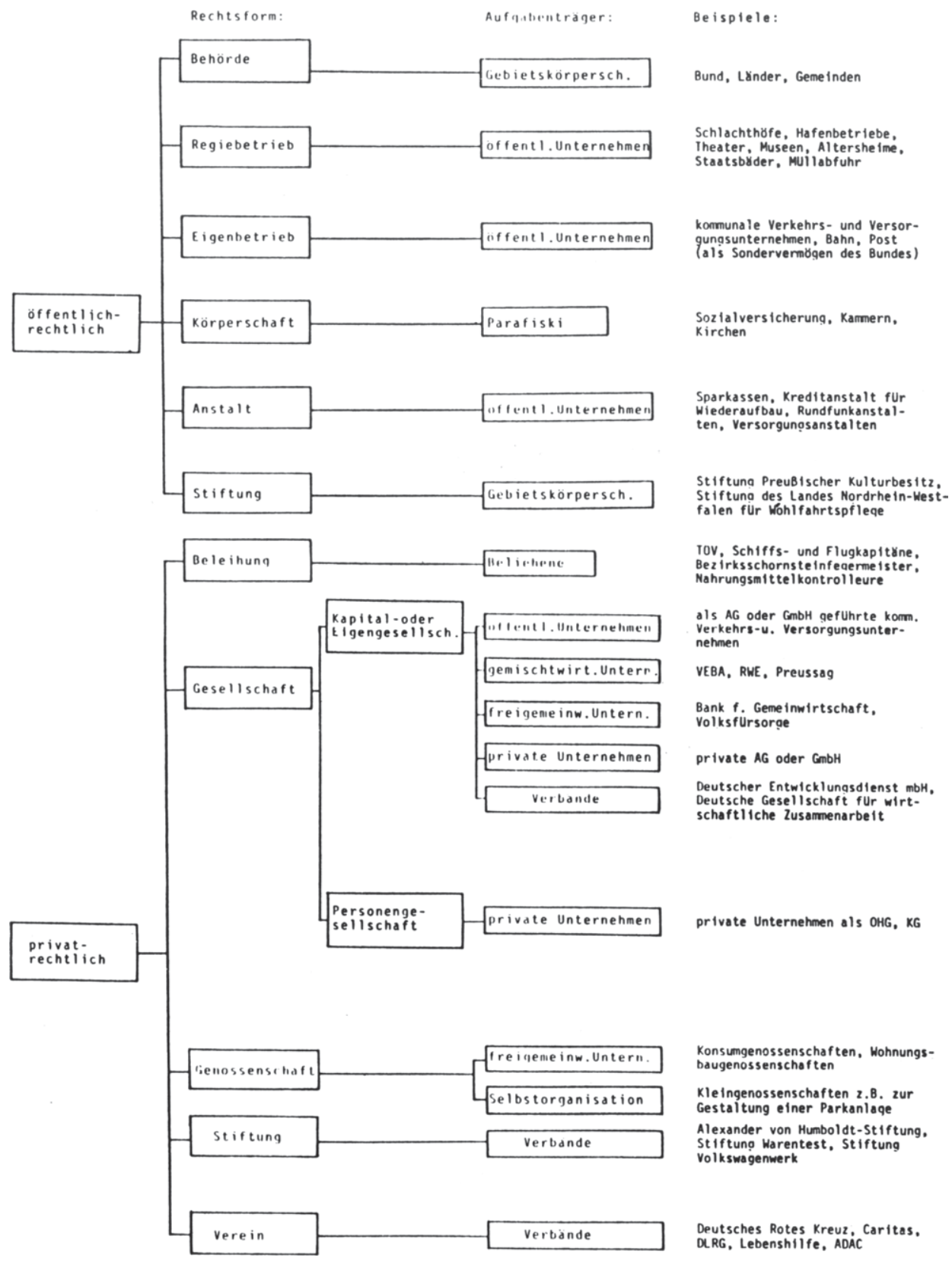


öffentlichen Versorgungsunternehmen in privatrechtlicher Form durchgeführt werden, hätte ein solches Vorgehen zur Folge, daß die gleiche ökonomische Leistung in der einen Gemeinde den Rang einer öffentlichen Aufgabe besäße, in einer anderen jedoch als private Aufgabe zu bezeichnen wäre. Selbst innerhalb einer Gemeinde würden gleiche Aktivitäten unterschiedlich erfaßt, wenn eine Kommune z.B. ein Schwimmbad in einem Stadtteil in eigener Regie, in einem anderen Stadtteil dagegen als GmbH betreibt.

Aus wirtschaftswissenschaftlicher Sicht kann die ZweckmäBigkeit einer Klassifizierung der Unternehmen anhand des Kriteriums der Rechtsform auch grundsätzlich in Frage gestellt werden. Die Rechtsformen liefern lediglich den formal-organisatorischen Rahmen für das Betätigungsfeld der Wirtschaftseinheiten. Sie sind so elastisch, daß weder über den ökonomischen Charakter der Unternehmen noch über den faktischen Einfluß ihrer Träger eindeutige Aussagen getroffen werden können. 1) Die Rechtsform ist deshalb vielmehr als Instrument der Unternehmenspolitik aufzufassen, während sie als Abgrenzungskriterium zwischen "öfféntlich" und "privat" ökonomisch bedeutsame Unterschiede zwischen den Unternehmen eher verdeckt. Daher ist auch KARL OETTLE zuzustimmen, der die Meinung vertritt, daß "zahlreiche rechtliche Organisationsmerkmale ihren realen Bedeutungswert erst von den jeweils vorliegenden ökonomisch-sozialen Verhältnissen empfangen, und daß sie mehrdeutig sind, insoweit diese 'Hintergrundverhältnisse' unbekannt bleiben."2) Im Zusammenhang mit der Privatisierungsdebatte wird aus diesem Grunde die übertragung von bisher durch Regie- oder Eigenbetriebe des Staates wahrgenommener Aufgaben auf öffentliche Unternehmen in privatrechtlichen Oraanisationsformen lediglich als formale Privatisierung bezeichnet. 3 )

1) Vgl. Th. Thiemeyer, a.a.0., S. $34 \mathrm{f}$.

2) K. Oettle, Die ökonomische Bedeutung der Rechtsform öffentlicher Betriebe, AöfU 8.Jg. (1967), S. 201.

2) $\mathrm{Vgl}$. Wissenschaftlicher Beirat be im BMF, Zur Lage und Entwicklung der Staatsfinanzen in der Bundesrepublik Deutschland, a.a.0., S. 1008. 
Als ein Argument für eine solche Aufgabenübertragung wird dann häufig eine höhere Flexibilität im Geschäftsgebaren privatrechtlich organisierter Wirtschaftseinheiten genannt. Betont werden muß allerdings, daß der Charakter einer Aufgabe durch die Änderung der Rechtsform, in der sie erfüllt wird, i.d.R. unberührt bleibt.

Ebenfalls Schwierigkeiten bereitet die sachgerechte Behandlung der Beliehenen. Selbst wenn ihr Auftreten als Subjekt des Privatrechts als hinreichende Bedingung für die Zuordnung zum privaten Sektor und damit als Träger privater Aufgaben angesehen würde, zeigt sich nur erneut die Unzulänglichkeit der Rechtsform als Abgrenzungskriterium. Die von Schiffs- und Flugkapitänen ausgeübte polizeiliche Gewalt an Bord wäre demnach als private Aufgabe zu betrachten, würde hingegen die gleiche Tätigkeit von einem Polizisten vorgenommen, handelte es sich um eine öffentliche Aufgabe.

Bedenken gegen die Rechtsform als adäquates Abgrenzungskriterium ergeben sich auch bezüglich weiterer Aufgabenträger. Verwiesen sei hier beispielhaft auf die Wirtschaftsforschungsinstitute, die zum Teil privatrechtlich und teilweise öffentlich-rechtlich tätig werden. 1) Schließlich bleibt die Wahl der Rechtsform als Abgrenzungskriterium aus ökonomischer sicht auch deshalb unbefriedigend, weil sie jederzeit durch eine politische Entscheidung geändert werden kann. In der Zeit von 1964 bis 1970 wurden beispielsweise 25 kommunale Unternehmen von der Rechtsform des Eigenbetriebes in die Form einer Eigengesellschaft umgewandelt. So wurden z.B. die Stadtwerke in Aachen, Duisburg und Bremerhaven in eine Aktiengesellschaft überführt, während u.a. Braunschweig, Koblenz und Münster eine überführung ihrer Eigenbetriebe in die Rechtsform der GmbH vor-

1) Während das Ifo, DIW- und RWI-Institut sich als eingetragene Vereine konstituiert haben, arbeiten das HWWA und das kieler Institut für Weltwirtschaft in der Rechtsform einer öffentlich-rechtlichen Anstalt; val. dazu auch F. Muiller-Thoma, a.a.0., S. 35. 
nahmen. 1) Ein weiteres Beispiel bietet das Deutsche Rote Kreuz, das bis 1937 als eingetragener Verein aktiv war, danach als öffentliche Körperschaft verfaßt wurde und sich nach seiner Auflösung im Jahre 1945 dann 1950 erneut als eingetragener Verein konstituierte. 2 )

Zusammenfassend läßt sich damit feststellen, daß die gleiche wirtschaftliche Tätigkeit in verschiedenen Rechtsformen stattfinden kann, daß die Rechtsform im Zeitablauf Veränderungen u.a. aus historisch-politischen Gründen unterliegt und daß die ökonomische Relevanz der Rechtsform nicht besonders hoch zu veranschlagen ist. Sie mag Auskunft über die Flexibilität in der Unternehmensführung geben oder ganz allgemein Informationen über die Kontrollmöglichkeiten des Staates 1 iefern; über Art und Wesen der Betätigung läßt sich jedoch allein mit Hilfe der Rechtsform kaum etwas aussagen. Das Auftreten eines Aufgabenträgers in öffentlich-rechtlicher oder in privatrechtlicher Organisationsform ist somit nicht bestimmend dafür, ob öffentliche oder private Aufgaben erfüllt werden.

\subsection{Die Bedeutung der Eigentumsverhältnisse}

Eine alternative Möglichkeit der institutionellen Aufgabenabgrenzung besteht darin, alle jene Institutionen, die sich im Statseigentum befinden, als Träger öffentlicher Aufgaben zu behandeln. Insbesondere im Rahmen der Diskussion über eine Privatisierung öffentlicher Unternehmen werden die Eigentumsverhältnisse vielfach zum alleinigen Entscheidungskriterium für Art und Weise der Aufgabenerfüllung erhoben, d.h. es wird unterstellt, daß mit der Veräußerung der Kapitalanteile des Staates an Private auch gleichzeitig der privatwirtschaft-

1) Vgl. F. Vogt, Die Bedeutung der Rechtsform für die Einflußmöglichkeiten der Gemeinden auf die Zielbildung kommunaler Versorgungsbetriebe, daraestellt am Beispiel des Eigenbetriebes und der Eigengesellschaft, Diss., Mannhe im 1970, S. 1.

2) Vgl. K. Blaum, Rotes Kreuz, HdSW 9. Bd., Göttingen 1956, S. 44 sowie F. MintTer-Thoma, a.a.0., S. 35. 
liche Charakter der Unternehmenstätigkeit vollständig determiniert sei. Umgekehrt wird dann argumentiert, bereits das staatliche Eigentum an Produktionsmitteln begründe eine besondere - von der einer ausschließlich von privaten Anteilseigneren geführten Unternehmung abweichenden - Zielsetzung, nämlich der Erfüllung spezieller öffentlicher Aufgaben. Bieten also die Eigentumsverhältnisse der Aufgabenträger das objektive Abarenzungskriterium, mit dessen Hilfe öffentliche und private Träger und damit auch öffentliche und private Aufgaben eindeutig unterschieden werden können? Zumindest läßt die Form des Eigentums in den meisten Fällen problemlos eine Identifikation der Träger als öffentlich bzw. privat zu. Gebietskörperschaften gehören per se zum staatlichen Eigentum, und öffentliche Unternehmen befinden sich ex definitione wiederum ganz oder mehrheitlich im Eigentum der Gebietskörperschaften. Ebenso problemlos ist die Zuordnung der Beliehenen, der privaten Unternehmen, der freien Berufe etc. zum privaten Sektor, da die Bereitstellung von Gütern und Dienstleistungen in diesen Fällen ausschließlich aufgrund des Zusammenwirkens privater Produktionsmittel erfolgt.

In zweifacher Hinsicht erweist sich aber das Kriterium Eigentumsverhältnisse als wenig geeignet für eine Aufgabenabgrenzung.

Erstens versagt auch das Eigentum in einigen Fällen als Zurechnungsmaßstab. Probleme ergeben sich einmal dort, wo privates Kapital aufgeht in das Eigentum einer Gemeinschaft. Dies ist immer dann der Fall, wenn Private sich zusammenschlieBen zu Verbänden, Vereinen oder wenn private Gelder einer Stiftung zugefüht werden. Das Eigentum verliert hier seine scharfen Konturen als Abgrenzungskriterium für öffentliche und private Aufgabenträger. Zum anderen tauchen Zuordnungsschwierigkeiten auch dort auf, wo - wie im Falle der gemischtwirtschaftlichen Unternehmung - öffentliches und privates Kapital in irgend einem Verhältnis zusammenwirken. 
Zweitens werden ungeachtet einiger Grenzfälle, die eine Bestimmung der Eigentumsverhältnisse erschweren, auch grundsätzliche Vorbehalte gegen eine ausschließliche Verwendung der Eigentumsstruktur als Abgrenzungskriterium erhoben. Unschwer kann gezeigt werden, daß sich solche Bedenken vor allem im Hinblick auf die Behandlung der öffentlichen Unternehmen als nicht unbegründet erweisen. Augenscheinlich gibt es Unternehmen, die, obwohl sie sich völlig im Eigentum der öffentlichen Hand befinden, durchaus in einem marktwirtschaftlichen Bezugssystem stehen und ebenso agieren wie Gesellschaften, die allein von Privateigentümern betrieben werden. Die bloße Orientierung an das Eigentum an Produktionsmitteln hat aber zur Folge, daß sämtliche öffentlichen Unternehmen, d.h. auch alle rein erwerbswirtschaftlich ausgerichteten Wirtschaftseinheiten wie etwa die Lufthansa oder die Stahlwerke Salzgitter dem öffentlichen Sektor zugeordnet werden. Eine solche Abgrenzung muß aus wirtschaftlicher Sicht unbefriedigend bleiben, verschleiert doch die Betonung der Eigentumsform den Blick für die ökonomisch wesentlich bedeutsamere Tatsache, daß nämlich nicht notwendigerweise Unterschiede in der Zielsetzung öffentlicher und privater Unternehmen bestehen müssen. Konsequenterweise begründet KARL OETTLE deshalb den öffentiichen Charakter von Unternehmen nicht mit Hilfe der Eigentumsverhältnisse, sondern in erster Linie mit ihren speziell öffentlich-wirtschaftlichen Zielsetzungen. Nur jene Unternehmen in staatlichem Eigentum, die eben diese Ziele verfolgen, sieht er als öffentliche Betriebe an. 1) Ein solches Vorgehen hat jedoch unglücklicherweise wiederum den entscheidenden Nachteil, daß eine Gegenüberstellung öffentlich-wirtschaftlicher und privatwirtschaftlicher Zielsetzungen zwangsläufig zu neuen Unschärfen führen muß.

1) Vgl. K. Oettle, Über den Charakter öffentlich-wirtschaftlicher Zielsetzungen, ZfbF N.F., 18. Jg. (1966), S. 246. 
Im Zusammenhang mit den öffentlichen Unternehmen ergeben sich noch aus einem weiteren Grund Bedenken gegen eine zu starke Betonung der Eigentumsverhältnisse als Abgrenzungskriterium. Unabhängig davon, welche Bedeutung den öffentlichen Unternehmen als Instrument der Wirtschaftspolitik uberhaupt beigemessen wird 1), gibt die Eigentumsstruktur noch nicht notwendig bereits darüber Auskunft, inwieweit Unternehmen im Eigentum der öffentlichen Hand tatsächlich als Instrument staatlicher Politik nutzbar gemacht werden. Eventuell greift der Staat mittels Gesetzen und Auflagen wesentlich weitgehender in das Marktgeschehen ein als infolge eines Eigentumerwerbs, so daß mit der Kenntnis der im staatiichen Eigentum befindlichen Unternehmen noch nicht die Frage nach der durch den Staat ausgeübten Kontrolle auf diese Unternehmen beantwortet ist.

Obgleich in der politischen Auseinandersetzuna die Eigentumsfrage weiterhin eine große Rolle spielt, wird ihre Relevanz in der Wirtschaftswissenschaft zunehmend als gering erachtet. 2) Statt dessen stellt man nun vornehmlich auf die Ausgestaltung der Verfügungsmacht über das Eigentum ab, d.h. der öffentlichwirtschaftliche oder privatwirtschaftliche Charakter eines Unternehmens bestimmt sich danach, ob die betriebliche Planungsund Entscheidungsgewalt zentral von der statichen Wirtschaftsbehörde ausgeübt wird oder aber in dezentralisierter Form erfolgt. Dominieren in einem öffentlichen Unternehmen ähnlich dezentrale Entscheidungsmechanismen wie in einem privaten Unternehmen, so fügen sich auch Unternehmen in Staats-

1) Vgl. Th. Thiemeyer, a.a.0., S. 28 m.w.N.; W. Hamm, Kollektiveigentum, Heidelberg 1961, S. $174 \mathrm{ff}$.; A. van der Bellen, Offentliche Unternehmen zwischen Markt und Staat, Köln 1977, S. $18 \mathrm{ff}$.

2) Diese Entwicklung ist insoweit bemerkenswert, da in der älteren ökonomischen Literatur fast durchgängig die herausragende Bedeutung der Eigentumsverhältnisse betont wird, in den Schriften marxistischer Provenienz die Ausgestaltung der Eigentumsverhältnisse sogar zur Kernfrage erhoben wurde.

Besonders deutlich wird die sekundäre Bedeutung der Eigentumsstruktur auch im europäischen Gemeinschaftsrecht dokumentiert. Da Art. 222 EWGVertrag die Eigentumsordnung in den verschiedenen Mitgliedstaaten der Gemeinschaft unberührt läßt, kann daraus geschlossen werden, daß die Errichtung einer supranationalen Wirtschaftsordnung unabhängig von der Regelung der Eigentumsfrage möglich ist; vgl. dazu G. Puittner, a.a.0., S. 25 und S. $280 \mathrm{ff}$. 
eigentum reibungsios in das marktwirtschaftliche system ein. 1)

\section{Die Kompetenzstruktur der Aufgabenträger}

Bisher wurde im Zusammenhang mit den Aktivitäten der Aufgabenträger recht allgemein von "Wahrnehmung" bestimmter - öffentlicher oder privater - Aufgaben gesprochen und dabei unterstellt, die Erfüllung einer Aufgabe sei als ein nicht teilbarer Komplex zu betrachten, die deshalb auch jeweils nur durch einen einzelnen - öffentlichen oder privaten - Träger erfolgen könne. Wird aber vermutet, daß für die Bestimmung des ökonomischen Charakters einer Aufgabe die Beantwortung der Frage nach dem Träger der Planungs- und Entscheidungskompetenz von größerer Bedeutung ist als die Kenntnis der Eigentumsverhältnisse, dann ist es zweckmäßig, das Kriterium Trägerschaft zu präzisieren, um auf diesem Wege weitergehende Informationen für eine Aufgabenabgrenzung zu gewinnen.

Bereits die tägliche Erfahrung vermittelt den Eindruck, daß eine organisatorische Kompetenzverteilung des Gesamtkomplexes "Aufgabenwahrnehmung" auf mehrere Träger eher die Regel als die Ausnahme ist. So zählen Maßnahmen zur Energieeinsparung heute in der Bundesrepublik zu den vorrangigen Aufgaben, ohne daß sie als öffentliche oder private identifiziert sind. Wird die Trägerschaft zum Abgrenzungskriterium erhoben, ist die Antwort davon abhängig, ob ein öffentlicher oder privater Träger diese Aufgabe "wahrnimmt". Die "Wahrnehmung" wird lediglich zum Oberbegriff einer Mehrzahl von unterschiedlichen Trägern ausgeführten Aktivitäten. Werden durch ein Gesetz spezielle Isolierungsmaßnahmen für Neubauten vorgeschrieben, so obliegt die Durchführung und Finanzierung dieser Maßnahmen den Bauherren, d.h. Durchführungs- und Finanzierungsträger der Aufgabe Energieeinsparung sind Private. ${ }^{2)}$ Ihnen ist somit nicht freigestellt,

1) $\mathrm{Vgl}$. K. Hax, Die öffentliche Unternehmung in der Marktwirtschaft, in: Finanzarchiv, N.F., Bd. 27 (1968), S. 47.

2) Von der Möglichkeit, daß auch der Staat als Bauherr auftreten kann, sei abgesehen. 
selbst darüber zu entscheiden, ob sie Energieeinsparungen durch Isolierungsmaßnahmen als ihre Aufgabe betrachten oder nicht, sondern die Entscheidungskompetenz liegt beim Staat. Er bestimmt, ob Maßnahmen vorzunehmen sind und in welcher Form dies zu geschehen hat. Darüber hinaus kontrolliert er die Durchführuna der Maßnahmen. Entscheidungs- und Aufsichtsträger ist demnach der Staat. Eine andere Kompetenzverteilung der Aufgabe Eneraieeinsparung läge vor, wenn Einsparungsmaßnahmen über finanzielle Anreize des Staates initiiert werden. Da der Bauherr selbst darüber entscheiden kann, ob er die subventionierten Isolierungsmaßnahmen vornehmen will oder nicht, verbleibt die Entscheidungsgewalt bei den privaten Trägern, während der Staat durch die öbernahme eines Teils der Kosten jetzt zusammen mit den Privaten zum Finanzierungsträger wird. Schließlich besteht eine weitere Möglichkeit der Kompetenzaufspaltung zwischen öffentlichen und privaten Trägern darin, daß der Staat durch die Verbreitung von Energiesparappellen lediglich als Informationsträger fungiert. Private sind dann in vollem Umfange sowohl Entscheidungs-, Durchführungs- und Finanzierungsträger. 1)

Als "Organisationsfunktionen" der Aufgabenwahrnehmung können demnach Entscheidung, Durchführung, Finanzierung, Aufsicht und Informationsbereitstellung unterschieden werden. ${ }^{2}$ ) Erfolgt eine Zuordnung dieser Funktionen zu den einzelnen Trägertypen (Obersicht 8) so wird deutlich, daß eine weitgehende, auf die Kompe-

1) In Form von Informationsbereitstellungskosten verursacht auch ein Appell Kosten auf Seiten des öffentlichen Trägers. Diese sollen zunächst genauso vernachlässigt werden wie die anfallenden Gemeirkosten bei der Gesetzgebung.

2) Bewußt wird hier mit dem Begriff "Organisationsfunktion" eine Bezeichnung gewählt, die sich von der in der Betriebswirtschaftslehre üblichen Gliederung der Organisation der Leistungserstellung u.a. in Phasen (Planung, Durchführung, Kontrolle) und Rang (Entscheidung und Ausführung) abhebt; vgl. dazu E. Kosiol, Organisation der Unternehmung, Wiesbaden 1962, S. $49 \mathrm{ff}$. Eine GTiederung nach Phasen betont u.E. zu sehr die zeiti iche Abfolge des Betriebsprozesses, während die Ranggliederung mit ihrer Zweiteilung in Entscheidung und Ausführung zu kurz greift und deshalb wichtige Aspekte vernachlässigt. 
Obersicht 8: Schematische Darstellung der Kompetenzstruktur der Aufgabenträger

\begin{tabular}{|c|c|c|c|c|c|}
\hline $\begin{array}{l}\text { Kompetenz- } \\
\text { trägaber }\end{array}$ & $\begin{array}{l}\text { Entschei- } \\
\text { dungs- } \\
\text { träger }\end{array}$ & $\begin{array}{l}\text { Durchfüh- } \\
\text { rungs- } \\
\text { träger }\end{array}$ & $\begin{array}{l}\text { Finanzie- } \\
\text { rungs- } \\
\text { träger }\end{array}$ & $\begin{array}{l}\text { Aufsichts- } \\
\text { träger }\end{array}$ & $\begin{array}{l}\text { Informa- } \\
\text { tions- } \\
\text { träger }\end{array}$ \\
\hline $\begin{array}{l}\text { Gebietskörper- } \\
\text { schaften }\end{array}$ & 1 & 1 & 1 & 1 & 1 \\
\hline Parafiski & 2 & 2 & 2 & 2 & 2 \\
\hline Beliehene & 3 & 3 & 3 & 3 & 3 \\
\hline $\begin{array}{l}\text { öffentliche } \\
\text { Unternehmen }\end{array}$ & 4 & 4 & 4 & 4 & 4 \\
\hline $\begin{array}{c}\text { private } \\
\text { Unternehmen }\end{array}$ & 5 & 5 & 5 & 5 & 5 \\
\hline freie Berufe & 6 & 6 & 6 & 6 & 6 \\
\hline $\begin{array}{l}\text { Selbstorganisa- } \\
\text { tion Privater }\end{array}$ & 7 & 7 & 7 & 7 & 7 \\
\hline $\begin{array}{l}\text { private } \\
\text { Haushalte }\end{array}$ & 8 & 8 & 8 & 8 & 8 \\
\hline
\end{tabular}

tenzregelung abgestellte Präzisierung der Trägerschaft möglich ist. 1)

1) Ansätze einer Präzisierung der Aufgabenträgerschaft finden sich auch bei W. Kirberger, der zwischen Ausgaben- und Entscheidungsträner trennt, sowie bei R. L. Frey, der Angebots- und Finanzierungsträger unterscheidet; val. W. Kirberger, a.a.0., S. $61 \mathrm{ff} . ;$ R. L. Frey, Infrastruktur, 2. Aufl., Tübingen 1972, S. $89 \mathrm{ff}$. Der Intention nach auch H. Zimmermann, Die Ausgabenintensität der öffentlichen Aufgabenerfülluna, in: Finanzarchiv, N.F., Bd. 32 (1974), S. $1 \mathrm{ff}$. 
Das Beispiel "Energieeinsparung durch Isolierungsmaßnahmen in Neubauten privater Haushalte" kann dann gemäß der übersicht 8 durch alternative Zahlenkombinationen gekennzeichnet werden. Folgende Möglichkeiten sind denkbar:

1.4 .1 .1 .1$.

1.5 .1 .1 .1$.

1.5 .8 .1 .1$.

$8 \cdot 5 \cdot(1+8) \cdot 8 \cdot 1$.

$8 \cdot 5 \cdot 8.8 \cdot 1$

$8 \cdot 5.8 .8 .8$

$8 \cdot 8 \cdot 8 \cdot 8.8$

uber allgemeine Deckungsmittel (z.B. Steuern) finanzierte Durchführung von Isolierungsmaßnahmen in eigener Regie des Staates

über allgemeine Deckungsmittel finanzierte Vornahme von Isolierungsmaßnahmen durch Auftragsvergabe des Staates an private Unternehmen

gesetzliche Verpflichtung der privaten Haushalte zur Vornahme von Isolierungsmaßnahmen ohne finanzielle Beteiligung des Staates

finanzielle Beteiligung des Staates an der Vornahme von Isolierungsmaßnahmen durch private Haushalte

Appelle von seiten des States an private Haushalte, Isolierungsmaßnahmen vorzunehmen

freiwillige Vornahme von Isolierungsmaßnahmen durch private Haushalte in eigener Verantwortung

freiwillige Vornahme von Isolierungsmaßnahmen durch private Haushalte in eigener Verantwortung und in eigener Regie (do-ityourself)

Unter institutionellen Gesichtspunkten und bei Spezifizierung der Trägerschaft kann eine Aufgabe demnach eindeutia nur dann als öffentlich (privat) charakterisiert werden, falls sämtli- 
che Kompetenzen ausschließlich von öffentlichen (privaten) Trägern ausgehen. In der überwiegenden Zahl der Fälle werden Mischformen zu beobachten sein, die sich durch ein differenziertes Zusammenwirken öffentlicher und privater Träger bei der Aufgabenerfüllung auszeichnen. Wo verläuft aber dann die Grenze zwischen privater und öffentlicher Aufgabenerfüllung? Als ein mög1 icher Lösungsweg bietet sich an, eine Aktivität immer dann als öffentliche Aufgabe zu betrachten, wenn die Mehrzahl der Organisationsfunktionen durch öffentliche Träger erfüllt wird, d.h. die Mehrzahl der Kompetenzen öffentlichen Trägern zufällt. Ein solches Vorgehen geht allerdings von der Annahme aus, daß sämtliche Organisationsfunktionen in gleichem Maße einen Beitrag zur Aufgabenbestimmung leisten. Demgegenüber darf aber vermutet werden, daB den einzelnen Funktionen ein sehr unterschiedliches Gewicht im Hinblick auf eine Abgrenzung der Aufgaben zukommt. Im folgenden soll deshalb die Kompetenzstruktur der Aufgabenträger näher charakterisiert, insbesondere ihre Bedeutung für eine Aufgabenzuordnung herausgestel1t werden.

\subsection{Informationsträger}

Je nach Art und Weise der Aufgabenformulierung kann die Bereitstellung von Informationen in zweifacher Hinsicht Gegenstand der Betrachtung sein. Wird die "gesetzliche Verpflichtung der Zigarettenhersteller, auf Zigarettenschachteln über den Schadstoffgehalt ihrer Produkte $z u$ informieren" als eine gesundheitspolitische Aufgabe formuliert, dann ist bereits die Informationsbereitstellung selbst als eigenständige Aufgabe anzusehen. In diesen Fällen ist es möglich, die Aufgabe "Informationsbereitstellung" wiederum in Organisationsfunktionen zu zerlegen. Den Zigarettenherstellern wird die Durchführung und Finanzierung der Aufgabe überantwortet, während der Staat als Entscheidungs- und Aufsichtsträger fungiert. Wird dagegen die gesundheitspolitische Aufgabe mehr zielorientiert in der Verringerung der Krankheits- und Todesfälle durch Zigarettenkonsum gesehen, so ist die Informationsbereitstelluna als ein organisatorischer Bestandteil der Aufgabenerfüllung aufzufassen. 
Führen staatliche Institutionen Aufklärungskampagnen durch oder appellieren sie an die Gesundheit der Raucher, so fungieren sie nur als Informationsträger. Sie mögen u.U. auch den Entscheidungsprozeß der Raucher beeinflussen, ohne allerdings damit gleichzeitig Entscheidungsgewalt auszuüben. 1)

Die Bereitstellung von Informationen stellt im Vergleicn zu alternativen Instrumenten wie insbesondere Auflagen, Geund Verbote eine äußerst weiche Form der Verhaltensbeeinflussung der Wirtschaftssubjekte dar, aber auch die Einwirkungsmöglichkeiten im Rahmen der Informationsbereitstellung zeichnen sich ihrerseits wiederum durch unterschiedliche Intensitäten aus:

a) Informative Berichte, Pläne, Programme und Beratung

- Berichte (z.B. Jahreswirtschaftsberichte, Verkehrslageberichte)

- Absichtserklärungen (z.B. Regierungserklärungen, Parteiprogramme, Stellungnahmen von Verbänden)

- Orientierungsdaten ( $z$.B. Steuerschätzungen, Bildungsgesamtpläne)

- Beratung (z.B. Berufsberatung, Verbraucherberatung)

b) Moralische Oberredung

- Aufklärung (z.B. über Gefahren des Drogenmißbrauchs, Möglichkeiten der Krebsvorsorge)

- Appelle (z.B. Maßhalteappelle, Energiesparappelle)

c) Positive und negative ideelle Anreize

- Titel- und Ordensverleihung

- öffentliche Belobigung bzw. Rüge.

1) Auch in diesem Zusammenhang wird damit erneut der besondere Stellenwert der im ersten Teil, Abschnitt I.2 vorgenommenen Überlegungen im Hinblick auf eine sachgerechte Aufgabendimensionieruna deutlich. 
Welche Bedeutung kommt dem Träger von Informationen bezijglich einer institutionellen Aufgabenabgrenzung zu? Handelt es sich bereits um eine öffentliche Aufgabe, wenn die mit ihrer Erfiullung verbundene Informationsbereitstellung durch einen öffentlichen Träger erfolgt? Diese Frage ist zu bejahen, falls eine Aufgabe dann als statlich gilt, wenn der Stat sich mit ihr in irgendeiner Form befaßt. 1) Eine solche Grenzziehung ist jedoch in zweifacher Hinsicht unpräzise. Würde alles zur öffentlichen Aufgabe erklärt, was Gegenstand der Information durch öffentliche Träger ist, so hätte dies eine sehr extensive Formulierung öffentlicher Aufgaben zur Folge. Andererseits greift eine solche Abgrenzung aber auch zu kurz, unterstellt sie doch, daß die Informationsbereitstellung durch Private ausnahmslos der Erfuillung privater Tätigkeiten dient. Konsumentenberatung durch Verbraucherzentralen, Drogenberatung durch private Wohlfahrtsverbände, Appelle der Automobilclubs, sich während der Fahrt anzuschnallen, etc. sind aber Beispiele dafür, daß auch private Institutionen für die gleichen Aufgabenbereiche Informationen bereitstellen wie öffentliche Träger. Die Art der Informationsträgerschaft bietet deshalb allenfalls Indizien für die Intensität der Regelungsbedurftigkeit einer Aufgabe, sie besitzt jedoch allein keine prägende Wirkung für die Charakterisierung einer Aufgabe als öffentliche oder private.

\subsection{Durchführungsträger}

Bei Anwendung dieses Kriteriums würde eine Aufabe immer dann als öffentliche bezeichnet, wenn ihre Durchführung einem öffentlichen Träger obliegt. Unter Aufgabendurchführung wird hier das Tätigwerden im engeren Sinne, der eigentiiche Akt der Bereitstellung von Gütern und Dienstleistungen verstan-

1) In diesem Sinn hat das Bundesverfassungsgericht in dem Fernsehurteil u.a. seine Entscheidung begründet, vgl. BVerfGE 12, S. 243. 
den. 1) Unschwer kann gezeigt werden, daß die Kenntnis des Trägers der Aufgabendurchführung isoliert ebenfalls noch keine hinreichende Begründung für die Charakterisierung einer Aktivität als öffentliche oder private zu geben vermag. Neben der Durchführung öffentlicher Aufgaben durch öffentliche Träger kann auch Privaten die Durchführung auf dem Wege der Inpflichtoder Indienstnahme auferlegt werden. Ferner können Private zur Durchführung freiwillig bereit sein. Im einzelnen sind folgende Organisationsformen der Durchführung zu unterscheiden:

- Beleihung (z.B. technische Sicherheitsprüfung durch den TOV)

- gesetzliche Verpflichtung (z.B. Umweltschutzauflagen für Unternehmen, Straßenreinigung durch Anlieger)

- öffentliche Konzessionsvergabe (z.B. Nahverkehrslinien, Verund Entsorgungsunternehmen)

- öffentliche Auftragsvergabe (z.B. Reinigungsdienste, Fahrdienste)

- private Leistungsbereitschaft aufgrund finanzieller Anreize (z.B. private oder gemeinnützige Einrichtungen der Krankenund Altenpflege)

- private Leistungen in eigener Regie und Verantwortung ( $z$. B. familiäre Kranken- und Altenpflege, Kindererziehung).

Häufig wird bereits eine Ausgliederung der Aufgabendurchführung aus der allgemeinen Verwaltung und ihre obertragung auf private Träger als Privatisierung öffentlicher Aufgaben bezeichnet. 2 )

1) Die Bereitstellung ist dabei streng von der Produktion zu trennen. Die Produktion wird in einem marktwirtschaftlichen System häufig auch dann privaten Unternehmen überlassen, wenn die Bereitstellung der Produkte öffentlich erfolgt. Niemand wird beispielsweise ernsthaft den öffentlichen Charakter der Aufgabe Verteidigung allein deshalb in Frage stellen, weil erwerbswirtschaftlich orientierte private Unternehmen die Produktion von Militärguitern übernehmen und diese an den Staat veräußern. Die Notwendigkeit einer klaren Unterscheidung zwischen Bereitstellung und Produktion betont auch R.A.Musgrave, Provision for Social Goods, a.a.0., S. 124.

2) So ausdrücklich beispielsweise W. Kux, Ansätze zur Privatisierung öffentlicher Aufgaben, Institut für Kommunalwissenschaften der Konrad-AdenauerStiftung, St. Augustin 1976, S. 2 f.; im Zusammenhang mit der Konzessionierung vgl. auch Institut für angewandtes Wirtschaftsrecht e.V.; Privatisierung der Risiken?, JaW-Schriften 2, Múnchen 0.J., S. 54. 
Im Falle der Indienstnahme durch öffentliche Konzessions-oder Auftragsvergabe wird aber lediglich die Durchführung privatisiert, während die übrigen Organisationsfunktionen in der Hand öffentlicher Träger verbleiben. Genaugenommen könnte hier also lediglich von einer partiellen Privatisierung oder von einer "Durchführungsprivatisierung" gesprochen werden. Im Falle der privaten Leistungsbereitschaft aufgrund finanzieller Anreize oder der gesetzlichen Verpflichtung wird darüber hinaus auch die Finanzierung teilweise oder vollständig privatisiert. Eine vollständige Privatisierung liegt demgegenüber nur dann vor, wenn eine Aufgabe aus der direkten öffentlichen Verantwortung und Entscheidung entlassen und dem Marktmechanismus überantwortet wird. 1) Damit wird deutlich, daß auch die alleinige Verwendung der Durchführungsträgerschaft als Kriterium für eine Aufgabenabgrenzung unzureichend bleiben muß.

\subsection{Finanzierungsträger}

Bei Zugrundelegung des Finanzierungsträgers als Abgrenzungskriterium wird die Klassifizierung der Aufgaben als öffentliche oder private danach vorgenommen, ob die im Zuge der Aufgabenerfüllung anfallenden Ausgaben von öffentlichen oder privaten Trägern getätigt werden. ${ }^{2)}$ Die Verwendung der Ausgaben als Hilfsgröße für die Aufgabenerfüllung hätte vor allem rechentechnische Vorteile, da die öffentliche Ausgabentätigkeit unmittelbar u.a. aus den Haushaltsplänen und den Volkswirtschaftichen Gesamtrechnungen ersichtlich ist. Empirische Untersuchungen zur Entwicklung der Staatstätigkeit ziehen deshalb fast ausschließlich die budgetwirksamen Ausgaben als einen hinreichend genauen Indikator für die statliche Aktivität heran. ${ }^{3)}$ Im Zusammenhang

1) So auch E. Thiel, Zielgerechte Veränderungen der Wahrnehmung von Staatsaufgaben, in: Hamburger Jahrbuch für Wirtschafts- und Gesellschaftspolitik, 22. Jg. (1977), S. 64.

2) Dabei geht es nicht um die Ermittlung derjenigen Wirtschaftssubjekte, die letztlich die Finanzierungslast zu tragen haben (z.B. durch Preisüberwälzung).

3) So z.B. K.-H. Raabe, a.a.0., S. 37 ff.; H. C. Recktenwald, a.a.0., S. $407 \mathrm{ff}$. 
mit der im Rahmen der Diskussion über den Staatsanteil am Sozialprodukt immer wieder im Mittelpunkt des Interesses stehenden Staatsquote wurde allerdings bereits mehrfach auf die Unzulänglichkeit der öffentlichen Ausgaben als Maß für die gesamte staatliche Aktivität hingewiesen. 1) Denn einerseits nehmen staatliche Träger zwar Aufgaben wahr, die sie praktisch in dem Maße erfiullen wie öffentliche Ausgaben für diesen Zweck getätigt werden - dies gilt etwa für die Ausgaben Straßenbau, Kuistenschutz oder Verteidigung -, daneben tritt der Stat aber auch durch gesetzgeberische Maßnahmen (Gesetze, Rechtsverordnungen, Verwaltungsvorschriften etc.) in Erscheinung, die, abgesehen von den ohnehin nicht zurechenbaren Gemeinkosten, zu keinen öffentlichen Ausgaben führen ( $z$.B. bestimmte Änderungen des Handelsrechts). Die Wahl der Finanzierungsträgerschaft als Abgrenzungskriterium hätte zur Folge, daß einige - u.U. sehr bedeutsame - öffentliche Aufgaben gar nicht erfaßt würden. Bei Aufgaben, deren Durchführung der Staat mittels Gesetzen und Auflagen den Privaten überträgt, fallen die eigentlichen Ausgaben bei den Privaten an. Beispiele bilden die Auflagen für Unternehmen im Bereich des Umweltschutzes, die Verpflichtung der Arbeitgeber zur Lohnfortzahlung und das Gebot zur Beschäftigung behinderter Arbeitnehmer in privaten Unternehmen. Die Tätigkeiten des States rufen also in sehr unterschiedlichem Maße Ausgaben bei den öffentlichen Haushalten hervor, d.h. sie sind nicht in gleicher Weise ausgabenintensiv. Je geringer die Ausgabenintensität der öffentlichen Aufqabenerfülluna ist ${ }^{2}$ ), desto

1) Vgl. dazu vor allem K. Littmann, Definition und Entwicklung der Staatsquote, Göttingen 1975 sowie Wissenschaftlicher Beirat beim BMF, Gutachter zur Aussagefähigkeit staatswirtschaftlicher Quoten, ButTet in der Bundesregierung $\mathrm{Nr} .90$ vom 30.7.1976, S. $849 \mathrm{ff}$.

2) Vgl. dazu H. Zimmermann, Die Ausgabenintensität der öffentlichen Aufgabenerfuillung, a.a.0., S. $1 \mathrm{ff}$. Auf diesen Sachverhalt hat bereits 1857 Friedrich Benedikt Wilhelm v. Hermann aufmerksam gemacht. Die Tatsache, daß die Aufgabenerfüllung des Staates sich nur zu einem Teil in den öffentlichen Haushalten widerspiegelt, wurde von ihm als "versteckter Staatsbedarf" bezeichnet; vgl. W. von Hermann, Staatswirtschaftliche Untersuchungen, 2. Auf1., München 1970, S. 50. Vgl. auch K. D. Henke, Die mangelnde Aussagekraft der öffentlichen Finanzen als Indikator raumwirksamer Staatstätigkeit, in: Jahrbuch für Sozialwissenschaft, Bd. 25 (1974), S. $393 \mathrm{ff}$. 
mehr fallen die Träger der Ausgaben und der Aufoaben auseinander. 1) Eine Grenzziehung zwischen öffentlichen und privaten Aufgaben, die den Träger der mit der Aufgabenerfuillung verbundenen Finanzierungslasten zum Abgrenzungskriterium erhebt, führt in diesem Fall zu dem unbefriedigenden Ergebnis, daß die uiber gesetzgeberische Maßnahmen des Staates initiierten Aktivitäten im privaten Sektor ohne Ausnahme als private Aufgaben ausaewiesen werden.

Da die Erfuillung öffentlicher Aufgaben nicht automatisch mit der Finanzierung über das Budget einhergeht, ist auch der Umkehrschluß unzulässig, daß ein finanzielles Engagement der öffentlichen Haushalte in jedem Fall der öffentlichen Aufgabenerfüllung dient.

Ausfürlich wurde bereits an anderer stelle erörtert, daß zahlreiche öffentliche Unternehmen sich in ihrer Zielsetzuna nicht von privaten Unternehmen unterscheiden. Da die unternehmerische Tätigkeit der Betriebe im staatlichen Eigentum jedoch budgetwirksam wird, wïrde bei Anwendung der Finanzierunasträgerschaft als Zuordnungskriterium auch diese rein erwerbswirtschaftiche Betätigung zu dem Bereich öffentlicher Aufgaben gezählt.

1) Je geringer die Ausgabenintensität der Aufgabenerfüllung ist, desto weniger aussagefähig wird deshalb auch die Staatsquote als Indikator der Staatstätigkeit, da innerhalb des Zahlenwerks der Volkswirtschaftlichen Gesamtrechnungen wesentliche Aktivitäten des Staates unberücksichtigt bleiben. Insbesondere im Hinblick auf internationale oder zeitliche Vergleiche von Staatsquoten können dann kaum noch eindeutige Urteile gefällt werden, falls in verschiedenen Ländern oder im Zeitablauf die Ausgabenintensität staatlicher Aufgabenerfüllung variiert. So dürte es voreilig sein, den vergleichsweise niedrigen Staatsanteil eines Landes dahingehend zu werten, der Staat halte sich mit seinen Aktivitäten weitgehend zurück. Der Staat kann vielmehr in sehr ausgeprägtem Maße öffentliche Aufgaben erfuillen, wobei die niedrige Staatsquote lediglich zum Ausdruck bringt, daß in diesem Land vergleichsweise mehr Aufgaben mit Hilfe wenig ausgabeintensiver Gebote und Verbote wahrgenommen werden. 


\subsection{Entscheidungsträger}

Als Träger der Entscheidung können jene Handlungsträger bezeichnet werden, die mit der Kompetenz ausgestattet sind, die Entscheidung über die Vornahme einer bestimmten Aktivität selbständig zu treffen. Je nach Zugehörigkeit des so definierten Entscheidungsträgers zum öffentlichen bzw. privaten Sektor würden demnach Aufgaben als öffentliche bzw. private identifiziert. Die Entscheidungsträgerschaft weist gegenüber den bisher erörterten Zuordnungsmerkmalen den Vorteil auf, daß damit nicht mehr nur alternative Erfüllungsmodalitäten zu Abgrenzungskriterien erhoben werden, sondern am Kernelement der Aufgabe angeknüpft wird. 1) Darüber hinaus wird der Träger der Entscheidungskompetenz vielfach auch gleichzeitig über Art und Weise der Aufgabendurchführung und - finanzierung bestimmen. Die Entscheidungsgewalt verbleibt so z.B. immer dann bei dem öffentlichen Träger Staat, wenn dieser das Verhalten der Privaten durch Auflagen, Ge-oder Verbote steuert oder auf dem Wege der Konzessions-bzw. Auftragsvergabe Privaten die Durchführung bestimmter Aufgaben überträgt. Objekte der Inpflicht-oder Indienstnahme Privater durch den Staat wären somit eindeutig öffentliche Aufgaben. Private Aufgaben wären demgegenüber immer dann zu konstatieren, wenn Private in eigener Regie tätig werden oder aber der Staat lediglich Informationen bereitstellt, finanzielle Unterstuitzung gewährt oder beaufsichtigend tätig wird, der Staat private Träger also aus dem Bereich der direkten öffentlichen Entscheidungsgewalt entläßt und ihnen diese selbst uiberantwortet.

Die Aufgabenabgrenzung mit Hilfe des Kriteriums Entscheidungsträgerschaft kann allerdings ebenfalls zu fragwürdigen Ergebnissen führen. Zwar wird eine extensive und unpräzise Formulierung öffentlicher Aufgaben vermieden, gleichwohl scheint es

- bei allen Vorbehalten gegenüber einer ungeprüten Zuordnuna sämtlicher finanzieller Unterstützungsmaßnahmen des Staates

1) Vgl. dazu auch W. Kirberger, a.a.0., S. 66. 
zum Komplex der öffentlichen Aufgaben - zumindest problematisch, eine Aufgabenerfiullung, die mit Subventions- oder Transferzahlungen einhergeht, nur deshalb als private Aufgabe auszuweisen, weil die Entscheidungskompetenz einem privaten Träger überlassen bleibt. Wenn der Staat sich finanziell an der Erfilluna der Aufgaben beteiligt oder wenn er nur als Aufsichtsträger fungiert, sind damit in vielen Fällen auch Mitsprache-oder Kontrollrechte verbunden. Eine exakte Ermittlung des letztlich relevanten Entscheidungsträgers ist dann oftmals nicht mehr möglich. 1)

\subsection{Aufsichtsträger}

Der Hinweis auf die Mitsprache- und Kontrollrechte des Staates führt schlieBlich zu einer letzten nier zu erörternden Kategorie des Kriteriums Trägerschaft. Als Aufsichtsträger sind alle jene Wirtschaftssubjekte zu bezeichnen, denen die Oberwachung der Einhaltung gesetzlicher Regelungen obliegt und die in Ausübung ihrer Aufsichtstätigkeit mit konkreten Kontrollbefugnissen ausgestattet sind. Zu nennen sind das Kartellamt zur Oberwachung des Wettbewerbs, die Bundesaufsichtsämter für das Kreditwesen, die Gewerbeaufsicht oder Bauaufsicht. Die Aufsichtstätigkeit kann sich beziehen u.a. auf Ge- und Verbote, Auflagen, Genehmigungs- und Anzeigepflichten, Zulassungen, Lizenzierungen, Konzessionierungen oder der öffentlichen Bindung von Unternehmen (z.B. Aufsicht über Preise, Produktionsstandards). Offentliche und private Aufsichtsträger bedienen sich also vielfältiger, in der Eingriffsintensität abgestufter Formen der Beaufsichtigung öffentlichen und privaten Handelns. Im Unterschied zum Kriterium Entscheidungsträger wird damit aber nicht das Kernelement der Aufgabe, sondern lediglich eine ihrer Erfüllungsmodalitäten zum Abgrenzungsmerkmal erhoben. Folglich ist im Vergleich zur Entscheidungsträgerschaft die Prägnanz dieses Kriteriums wesentlich geringer. Eine weitreichende Aufsichtstätigkeit läßt schließlich jede Aktivität zur öffentlichen Aufgabe werden.

1) Vgl. W. Kirberger, a.a.0., S. 67. 


\section{Erkenntniswert institutioneller Abgrenzungskriterien}

Da institutionelle Ansätze einer deskriptiven Bestimmung öffentlicher und privater Aufgaben auf formale Zuordnungskriterien abstellen, werden die im Rahmen der funktionalen Aufgabenabgrenzung auftretenden erheblichen Probleme einer materiellen Trennung zwischen privaten und öffentlichen Funktionen vermieden und auch die kaum lösbaren Schwierigkeiten umgangen, die immer dann auftreten, wenn eine Unterscheidung zwischen individuellem Nutzen und Gemeinwohl oder privatem und öffentlichem Interesse vorgenommen werden soll.

Die Typologie der Aufgabenträger vermittelt zunächst einen Eindruck davon, wer gegenwärtig in der Bundesrepublik Deutschland welche Aufgaben wahrnimmt. Es zeigt sich, daß die dichotomische Trennung $z$ wischen Staat und Markt, die immer noch die ökonomische Betrachtungsweise weitgehend beherrscht, den Blick für die Tatsache versperrt, daß überwiegend keine Kongruenz zwischen öffentlichen Trägern und öffentlichen Aufgaben einerseits und privaten Trägern und privaten Aufgaben andererseits besteht. Zwar wird im Regelfall die Bereitstellung von Konsumgutern von privaten Trägern vorgenommen, sie stellen somit private Aufgaben dar. Ebenso sind äußere Sicherheit und Rechtsprechung Beispiele für Aufgaben, die ausschließlich von öffentlichen Trägern erfiult werden und demnach als öffentliche Aufgaben zu klassifizieren sind. Daneben gibt es aber offenbar eine Vielzahl von Aufgaben, die weder als öffentliche noch als private identifiziert werden können, da sie jeweils sowohl von öffentlichen als auch von privaten Trägern wahrgenommen werden.

Die institutionelle Vorgehensweise zeigt weiter, daß die Art der Rechtsform oder die Ausgestaltung der Eigentumsverhältnisse allein keine geeigneten konstitutiven Merkmale für eine Aufgabenbestimmung darstellen. Die Rechtsform bildet lediglich 
einen weitgefaßten formal-organisatorischen Rahmen für das Handeln privater und öffentlicher Aufgabenträger, und der Charakter der Aufgabe bleibt von dem öffentlichen oder privaten Eigentum an Produktionsmitteln weitgehend unberüht.

Schließlich verdeutlicht die institutionelle Aufgabenabgrenzung einen dritten wichtigen Sachverhalt. Der Gesamtkomplex "Aufgabenwahrnehmung" kann in Organisationsfunktionen wie Information, Durchführung, Finanzierung, Entscheidung und Kontrolle aufgespalten und dementsprechend können Teilträgerschaften gebildet werden. Die Aufgabenerfuillung zeichnet sich dann im allgemeinen durch ein differenziertes Zusammenwirken öffentlicher und privater Teilträger aus. Der Trägerschaftsfrage kommt auch bei Privatisierungsansätzen eine besondere Bedeutung zu, sofern Privatisierung als Verlagerung einzelner oder mehrerer organisationsfunktionen von öffentijchen auf private Träger begriffen wird. Die Wahl der jeweils zweckmäßigen Träger einer Organisationsfunktion und das optimale Zusammenwirken der Durchführungs-, Finanzierungs - und Entscheidungsträger könnte für eine effektive und effiziente Aufgabenerfüllung entscheidend sein.

\section{Technisch-ökonomische Abgrenzungskriterien}

1. Das Konzept der öffentlichen Güter

In der neueren wirtschaftswissenschaftlichen, speziell aber der finanzwissenschaftlichen Diskussion über die Aufgabenverteilung zwischen dem privaten und dem öffentlichen Sektor ist der Begriff "öffentliche Güter" zum zentralen Stichwort avanciert.

Anknüpfend an Arbeiten italienischer, skandinavischer und deutscher Autoren Ende des vorigen Jahrhunderts ${ }^{1}$ ) waren es für die

1) Zu nennen sind insbesondere Mazzola, Sax, Wicksell, Lindahl und - allerdings später - Bowen. Auszüge aus den Werken einiger der genannten Autoren sind abgedruckt in R. A. Musgrave, A.T. Peacock (Hrsg.), Classics in the Theory of Public Finance, 4 . Aufi., New York 1967; $\mathrm{vgl}$. daneben $\mathrm{H}$. Bowen, The Interpretation of Voting in the Allocation of Resources, QJE Bd. 58 (1943), S. $27 \mathrm{ff}$. 
moderne Finanzwissenschaft vor allem PAUL A. SAMUELSON ${ }^{1}$ ) und RICHARD A. MUSGRAVE ${ }^{2}$, die maßgeblich zu einer wiederbelebung der Theorie der öffentlichen Güter beitrugen. Im Rahmen einer normativen Betrachtung über die Notwendigkeit der öffentlichen Durchführung bestimmter Aktivitäten zur Korrektur der Ergebnisse des marktwirtschaftlichen Prozesses entwarfen sie Konzepte zur Charakterisierung öffentlicher Giter.

Es ist zu prüfen, ob diesen Konzeptionen auch für die deskriptive Abgrenzung öffentlicher und privater Aufgabenbereiche ein Aussagewert beigemessen werden kann. Weist ein öffentliches Gut im Vergleich zu einem privaten Gut spezielle Eigenschaften auf, die eine öffentliche Bereitstellung zwingend erforderlich machen, und lassen sich diese Eigenschaften mit $\mathrm{Hilfe}$ objektiver Unterscheidungskriterien zweifelsfrei erfassen, so wäre es möglich, eine eindeutige Klassifizierung von Gütern und Dienstleistungen ${ }^{3}$ ) vorzunehmen in solche, die vom öffentlichen Sektor bereitgestellt, und solche, die vom privaten Sektor angeboten werden. Außerdem ist $z u$ prüfen, ob von einem öffentlichen Gut direkt auf eine öffentliche Aufgabe geschlossen werden kann, ob also bereits der Nachweis konkreter gutsspezifischer Eigenschaften gleichbedeutend ist mit der Identifikation einer öffentlichen oder privaten Aufgabe.

1) P. A. Samuelson, The Pure Theory of Public Expenditure, REStat Bd. 36 (1954), S. $387 \mathrm{ff}$.; derselbe, Diagrammatic Exposition of a Theory of PubIic Expenditure, REStat Bd. 37 (1955), S. 350; derselbe, Aspects of Public Expenditure Theories, REStat Bd. 40 (1958), S. $332 \mathrm{ff}$. Sowie derselbe, Pure Theory of Public Expenditure and Taxation, in: Public Economics, hrsg. von J. Margol is und H. Guitton, New York 1969, S. $98 \mathrm{ff}$.

2) R. A. Musgrave, The Theory of Public Finance, a.a.0.; derselbe, Provision for Social Goods, in: Public Economics, hrsg. von J. Margolis und $H$. Guitton, a.a.0., S. $124 \mathrm{ff}$. Sowie derselbe, Provision for Social Goods in the Market System, in: Public Finance, Bd. 26 (1971), S. $304 \mathrm{ff}$.

3) Obgleich in der angelsächsischen wie auch in der deutschsprachigen Literatur ganz überwiegend der Begriff "goods" bzw. "Güter" Verwenduna findet, sind damit doch in aller Regel auch die Dienstleistungen eingeschlossen. 


\subsection{Konstitutive Eigenschaften öffentlicher Güter}

Nach kontroverser Diskussion über die Charakteristika öffentlicher Güter, in deren Verlauf die grundlegenden Merkmale eines öffentlichen Gutes wiederholt verfeinert wurden ${ }^{1)}$, hat sich in der finanzwissenschaftlichen Literatur inzwischen weitgehend die Konvention durchgesetzt, ein öffentliches Gut durch zwei Eigenschaften zu kennzeichnen: der Nichtrivalität im Konsum und der Nichtanwendbarkeit des AusschluBprinzips. ${ }^{2}$ ) Uneinigkeit besteht aber weiterhin, ob diese beiden Merkmale selbständig konstitutiv wirken oder ob zur hinreichenden Definition eines öffentlichen Gutes beide Kriterien gemeinsam auftreten müssen. Außerdem ist umstritten, welche der beiden genannten Eigenschaften die grundlegende sei.

Ein typisch privates Gut wie z.B. ein Glas Bier, eine Waschmaschine oder ein Fahrrad ist zum einen dadurch gekennzeichnet, daß die Prinzipien des "do ut des" Gültigkeit besitzen, d.h. ein Wirtschaftssubjekt, welches ein privates Gut zu konsumieren wünscht, hat in Form der Zahlung des Kaufpreises eine Gegenleistung zu erbringen. Wer nicht bereit ist, einen Besitztitel zu erwerben, wird ausgeschlossen (Ausschlußprinzip). Für ein

1) Neben den bereits zitierten Arbeiten von Samuelson und Musgrave ist u.a. zu verweisen auf J. Margolis, A Comment on the Pure Theory of Public Expenditure, REStat Bd. 37 (1955), S. $347 \mathrm{ff} . ;$ G. Colm, Comments on Samuelson's Theory of Public Finance, REStat Bd. 38 (1956), S. $408 \mathrm{ff}$. sowie J. G. Head, Public Goods and Public Policy, in: Public Finance, Bd. 17 (1962), S. $197 \mathrm{ff}$. Einen überblick über diese Diskussion gibt K. N. Münch, Kollektive Güter und Gebühren. Elemente einer Gebührentheorie für Kollektivguiter, Göttingen 1976, S. $20 \mathrm{ff}$.

2) Vgl. K. Schmidt, Kollektivbeduirfnisse und Staatstätigkeit, in: Theorie und Praxis des finanzpolitischen Interventionismus. Fritz Neumark zum 70 . Geburtstag, hrsg. von H. Haller U.a., Tübingen 1970, S. 6. Allerdings ist darauf zu verweisen, daß gelegentlich die Theorie der öffentlichen Giiter als integraler Bestandteil eines generellen Externalitäten-Konzepts gesehen wird. In einem seiner späteren Aufsätze definiert z.B. Samuelson: "A public good ... is simply one with the property of involving a 'consumtion externality'..." P. A. Samuelson, The Pure Theory of Public Expenditure and Taxation, a.a.0., S. 102. Val. auch J.G. Head, The Theory of Public Goods, in: Rivista di Diritto Finanziario e Scienza delle Finanze, Bd. 27 (1968), S. 223 sowie derselbe, Externality and Public Policy, in: ebenda, Bd. 28 (1969), S. $383 \mathrm{ff}$. Ein solcher Ansatz ist für eine deskriptive Aufgabenabgrenzung jedoch kaum verwendbar; val. dazu dritter Teil, Abschnitt III, Punkt 1.4 dieser Arbeit. 
privates Gut ist zum anderen charakteristisch, daß es mit dem Konsum durch ein Wirtschaftssubjekt A gleichzeitig der potentiellen Verfügung eines Wirtschaftssubjektes $B$ entzonen wird (Rivalität). Im Gegensatz dazu liegt ein öffentliches Gut dann vor, wenn das Ausschlußprinzip nicht anwendbar ist und keine Rivalität im Konsum besteht. Als Paradebeispiele eines typisch öffentlichen Gutes dienen in der Literatur die nationale Verteidigung und der Leuchtturm. Konstatiert wird folgendes: Der Abwehreffekt der nationalen Verteidigung kommt allen Bürgern des Landes zugute, unabhängig davon, ob der einzelne bereit ist, einen Beitrag zu seiner Finanzierung zu leisten oder nicht; d.h. ein Ausschluß vom Konsum des Gutes Landes erteidigung ist nicht möglich. Auch besteht keine Rivalität zwischen den Konsumenten, da ein zusätzlicher Bürger Verteidigungsleistungen in Anspruch nehmen kann, ohne daß der Konsum der übrigen Bürger beeinträchtigt wird. Analoges gilt für Leuchttürme. Auch hier werden die Nutzer nicht um die Leuchtturmleistungen rivalisieren, und es besteht keine Möglichkeit, Nichtzahler vom Konsum der Leuchtturmleistung auszuschließen.

Besitzt ein Gut die genannten Eigenschaften, so ergeben sich daraus folgende Konsequenzen: Da der AusschluB einzelner Wirtschaftssubjekte von der Nutzung eines öffentlichen Gutes unmöglich ist, werden die Wirtschaftssubjekte selbst dann, wenn sie ein ausgeprägtes Bedürfnis z.B. nach innerer Sicherheit haben, bei der Offenlegung ihrer Präferenzen äuBerste Zurückhaltung üben, um nicht zur Leistung eines Finanzierungsbeitrages herangezogen zu werden. Sie nehmen die Haltung eines "Trittbrettfahrers" (free riders) ein, denn sie haben berechtigte Hoffnung, daß ein von ihnen präferiertes öffentliches Gut auch ohne ihren Beitrag bereitgestellt wird. Aufgrund ihres "Free-RiderVerhaltens" werden die Präferenzen der Wirtschaftssubjekte nicht in Nachfrage am Markt umgesetzt, folglich bestehen für private Produzenten auch keinerlei Anreize, öffentliche Güter am Markt anzubieten. 
Vielfach wird daraus geschlossen, daß immer dann, wenn Güter oder Dienstleistungen die gutstechnischen Eigenschaften der Nichtrivalität im Konsum und der Nichtanwendbarkeit des AusschluBprinzips aufweisen, notwendigerweise eine öffentliche Bereitstellung erfolgen muß. Dieser Schluß ist aber zumindest voreilig, denn nur in den seltensten Fällen liegen die Sachverhalte so eindeutig wie in den Lehrbuchbeispielen Landesverteidigung und Leuchtturm, und selbst diese sind nicht unumstritten.

\subsection{Anmerkungen zum Kriterium der Nichtrivalität im Konsum}

Häufig wird angenommen, daß die beiden Merkmale öffentlicher Güter selbständig konstitutiv wirken. 1) Wird aus der Existenz eines öffentlichen Gutes auf die Art der Bereitstellung geschlossen, so müßte demnach gefolgert werden, daß bereits das Vorhandensein einer der beiden begriffsbestimmenden Eigenschaften zwingend ein öffentliches Angebot erforderlich macht.

Offenbar kann aber eine Vielzahl von Fällen benannt werden, in denen zwar die Rivalitätsbedingung nicht erfüllt ist, gleichwohl aber eine Bereitstellung dieser Güter über den Markt auf keine Probleme stößt. So zeichnen sich beispielsweise Kinovorstellungen und Sportveranstaltungen dadurch aus, daß der Konsum der bereits anwesenden Besucher i.d.R. nicht beeinträchtigt wird, falls ein weiterer Besucher hinzukommt, vorausgesetzt es sind noch freie Plätze vorhanden. Da jedoch gleichzeitig das Ausschlußprinzip mit Hilfe von Eintrittskarten anwendbar ist, kann auch eine private Bereitstellung über den Marktpreis ohne Mühe praktiziert werden.

1) Auch Musgrave ist von seiner früheren Definition, nach der ein öffentliches Gut beide Eigenschaften zugleich aufweisen muß (vgl. The Theory of Public Finance, a.a.0., S. 10, Fußnote 1) in einem seiner späteren Aufsätze abgerückt, wenn er dort nunmehr davon ausgeht, daß bereits das Vorhandense in einer der beiden Eigenschaften zur hinreichenden Charakterisierung eines öffentlichen Gutes ausreicht; vgl. R. A. Musgrave, Provision for Social Goods, a.a.0., S. 126. 
Das Kriterium der Nichtrivalität selbst leidet darunter, daß es in den meisten Fällen nur innerhalb bestimmter Grenzen ailt. So herrscht z.B. auf einer Straße oder in einer Parkanlage nur solange keine Rivalität im Konsum, bis Kapazitätsgrenzen erreicht werden. 1) Bei normalem Verkehrsaufkommen wird sich der Benutzer einer innerstädtischen Straße kaum dadurch beeinträchtigt fühlen, daß diese Straße von weiteren kraftfahrzeugen befahren wird. Kommt es allerdings in Verkehrsspitzenzeiten zu straßenverstopfungen, so wird er gezwungen, auf Nebenstraßen auszuweichen oder aber Zeitverluste in Kauf zu nehmen. Treten Kapazitätsgrenzen auf, so kommt es zwischen den Autofahrern zur Rivalität im Hinblick auf die Nutzung der innerstädtischen Straße. Allerdings kann bereits vor Eintritt der Kapazitätsgrenzen partielle Rivalität zwischen den Konsumenten entstehen, da häufig mit zunehmender Benutzerzahl die Qualität eines angebotenen Gutes sich vermindert und damit individuelle Nutzeneinbußen auftreten. So wird z.B. der Erholungswert einer Parkanlage mit zunehmender Frequentierung für den einzelnen beträchtlich sinken. ${ }^{2}$ )

Das Merkmal der Nichtrivalität im Konsum erweist sich demnach in zweifacher Hinsicht als ein unzureichendes Unterscheidungskriterium für eine deskriptive Abarenzung öffentlicher von privaten Gütern. Erstens ist die zweifelsfreie Erfassung dieses Kriteriums als Voraussetzung für eine eindeutige Abgrenzung der Bereiche nicht erfüllt, da in der überwiegenden Zahl der Fälle die Nichtrivalität lediglich innerhalb bestimmter - früher oder später auftretender - Kapazitätsgrenzen Gültigkeit besitzt. Zweitens ist selbst die Gültigkeit der Nichtrivalitätsbedingung

1) Vgl. hierzu auch J. Margolis, a.a.0., S. 347 sowie J. G. Head, Public Goods and Public Policy, a.a.0., S. 201.

2) Auf diesen Sachverhalt hat insbesondere Head hingewiesen; J. G. Head, Public Goods and Public Policy, a.a.0., S. 202. Vgl. daneben die Untersuchunaen von W. H. Oakland, Congestion, Public Goods and Welfare, JPE Bd. 1 (1972), S. $339 \mathrm{ff}$. Sowie A. C. Deserpa, Congestion, Polution, and Impure Public Goods, in: Public Finance, Bd. 33 (1978), S. $68 \mathrm{ff}$. Deserpa unterscheidet explizit zwischen Ballung, Umweltverschmutzung und Warteschlangen als Ausprägungen der Qualitätseinbuße von Gütern infolge zunehmender Konsumentenzahlen. 
nicht in jedem Fall gleichbedeutend mit einer öffentlichen Bereitstellung der Güter und Dienstleistungen. Allein mit $\mathrm{Hilfe}$ der gutstechnischen Eigenschaft der Nichtrivalität im Konsum kann also keine hinreichende Charakterisierung öffentlicher Güter vorgenommen werden, d.h. eine selbständig konstitutive Wirkung ist diesem Kriterium nicht zuzuschreiben.

\subsection{Anmerkungen zum Kriterium der Nichtanwendbarkeit des} Ausschlußprinzips

Offentliche Güter sind deshalb erst hinreichend definiert, wenn neben der Nichtrivalität im Konsum gleichzeitig das Kriterium der Nichtanwendbarkeit des AusschluBprinzips vorliegt. ${ }^{1)}$ Allerdings bedarf auch dieses Kriterium der Präzisierung. Zu unterscheiden ist zwischen einer technologisch bedingten, einer ökonomisch begründeten und einer politisch erwünschten Nichtanwendung des Ausschlusses nichtzahlender Konsumenten. 2)

Die Möglichkeit des Ausschlusses wird einerseits durch technologische Bedingungen bestimmt. Da der Stand der Technik jedoch ständigen Wandlungen unterworfen ist, gilt das AusschluBprinzip nicht uneingeschränkt, sondern die Gültigkeit ist abhängig von Zeit und Raum. War es bisher nicht möglich, den Besitzer eines Fernsehgerätes vom Konsum der Sendungen auszuschließen, so sind mit der Entwicklung des Kabelfernsehens die technischen Voraussetzungen für eine Durchführung des Ausschlusses geschaffen. Technischer Fortschritt kann also eine Veränderung der Eigenschaften bestimmter Güter zur Folge haben. Mithin kann auch die technisch bedingte Notwendigkeit der öffentlichen Bereitstel-

1) So auch K. Schmidt, Kollektivbedürfnisse und Staatstätigkeit, a.a.0., S. 19.

2) Bödeker führt darüber hinaus eine juristische Begründung für die Unmöglichkeit der Durchführung des Ausschlußprinzips an. Das von ihm gewählte Beispiel zeigt jedoch bereits, daß die fehlende Durchsetzbarkeit von Eigentumsrechten auf die mit der Praktizierung des Ausschlußprinzips verbundenen zu hohen Kosten zurückzuführen ist, also letztlich ebenfalls ökonomisch bedingt ist; vgl. W. Bödeker, Allokations- und Distributionsprobleme bei Kollektivguitern, Meisenheim am Glan 1972, S. 13. 
lung von Gitern und Dienstleistungen zeitlich begrenzt sein. Mit Blickrichtung auf jene Fälle, in denen der Ausschluß gegenwärtig noch Schwierigkeiten bereitet, stellt deshalb auch MUSGRAVE fest: "In this case budgetary provision is ... needed, pending the development of a better 'exclusion technology'."1) HEAD geht noch einen Schritt weiter, wenn er bereits heute einen Ausschluß nie für völlig unmöglich hält. ${ }^{2}$ ) In der Tat werden inzwischen selbst klassische Beispiele öffentlicher Güter wie Verteidigung und Leuchtturm in Frage gestellt. So können zumindest Komponenten der Verteidigungsleistung bis zu einem gewissen Grade räumlich differenziert angeboten werden ( $z$. B. besonderer Schutz der Großstädte) ${ }^{3)}$, und das moderne Radarwesen stellt nicht nur eine bessere Alternative zu Feuerzeichen und Lichtsignalen dar, sondern ermöglicht auch den Ausschluß jener Schiffseigner, die nicht bereit sind, einen Finanzierungsbeitrag zu leisten. ${ }^{4}$ )

Zunehmend wird deshalb eine Nichtanwendung des Ausschlußprinzips ökonomisch begründet. ${ }^{5)}$ In manchen Fällen seien die AusschlieBungskosten so hoch, daß die - technisch durchaus mögliche Praktizierung des AusschluBprinzips zu Effizienzverlusten führe. Um solche Effizienzverluste zu vermeiden, sei dann die Bereit-

1) R. A. Musgrave, Provision for Social Goods in the Market System, a.a.0., S. 307.

2) Vgl. J. G. Head, Public Goods and Public Policy, a.a.0., S. 205.

3) Vgl. nierzu R. L. Frey, Infrastruktur, a.a.0., S. 73 ff.; R. H. Wlagner, National Defense as a Collective Good, in: Comparative PubTic Policy: Issues, Theories, and Methods, hrsg. von C. Liske U.a., New York 1975, S. $199 \mathrm{ff}$. Sowie die Untersuchungen von M. Jr. 01son, R. Zeckhauser, An Economic Theory of Alliances, REStat Bd. $48(1066), S .266 \mathrm{ff}$. und T. Sandler, Impurity of Defense: An Application of the Economics of AlTiances, in: Kyklos, Bd. 30 (1977), S. $443 \mathrm{ff}$.

4) Grundsätzlicher Art sind die Einwände von Coase. Er bemängelt zu Recht, daß die weite Verbreitung des Leuchtturmbeispiels allein deshalb möglich war, weil Wirtschaftswissenschaftler nie eine empirische Untersuchung über Angebot und Finanzierung von Leuchtturmleistungen für notwendig erachtet haben. Unschwer läßt sich nämlich z.B. für Großbritannien nachweisen, daß bis Mitte des 19. Jahrhunderts Leuchtturmleistungen durchaus profitabel von privaten Anbietern bereitgestellt wurden; vgl. R. H. Coase, The Lighthouse in Economics, JLawEc 1974, S. $357 \mathrm{ff}$.

5) So auch R. A. Musgrave, Provision for Social Goods in the Market System, a.a.0., S. 307 . 
stellung dieser Güter und Dienstleistungen über den öffentlichen sektor erforderlich.

Mit der Zugrundelegung einer relativen ökonomischen Unmöglichkeit des Ausschlusses durch Zuhilfenahme des Effizienzkriteriums wird jedoch die rein deskriptive Betrachtungsweise bereits verlassen. Selbst dann, wenn eindeutig entschieden werden könnte, in welchen Fällen die Anwendung des Ausschlußprinzips den Bedingungen der Effizienz genügt, so stellt die effiziente Allokation doch lediglich ein normatives Ziel dar, wobei implizit unterstellt wird, daß über die politische Wünschbarkeit der Realisierung der Allokationseffizienz bereits positiv entschieden wurde.

Das gleiche gilt, falls die Nichtanwendung des Ausschlußprinzips aus rein politischen Erwägungen für sinnvoll erachtet wird. Unter Effizienzgesichtspunkten mag z.B. die private Bereitstellung von Schulbildung hervorragende Ergebnisse liefern, dennoch können negative Erwartungen der Politiker im Hinblick auf die Chancengleichheit und die inhaltiche Gestaltung des Lehrangebots die Effizienzaspekte dominieren, so daß ein öffentliches Angebot ratsam erscheint, mithin auf die Anwendung des Ausschlusses verzichtet wird. Mittels der bloßen deskriptiven Momentaufnahme, die notwendigerweise die institutionellen Gegebenheiten als Datum setzen muB, kann dann zwar konstatiert werden, daß im Ergebnis eine. Differenz besteht zwischen der nach gutstechnischen Eigenschaften vorgenommenen Abgrenzung öffentlicher und privater Güter und der gemäß politischer Entscheidung sich ergebenden tatsächlichen Art und Weise der Güterbereitstellung, die - wesentlich interessantere - Analyse der Gründe für diese Abweichungen würden aber wiederum den Rahmen der deskriptiven Betrachtung sprengen. 


\subsection{Offentliche Güter und offentliche Aufgaben}

Selbst unter der heroischen Prämisse, öffentliche Güter seien durch gutstechnische Eigenschaften zweifelsfrei zu kennzeichnen, bliebe offen, inwieweit mit der Bestimmung öfentlicher und privater Güter gleichzeitig die Abgrenzung öffentlicher von privaten Aufgaben gelingt.

Unter Verzicht auf eine selbständige inhaltliche Festlegung fungiert der Ausdruck "Gut" ähnlich wie der Begriff "Aufgabe" häufig lediglich als Hilfselement zur Erschließung des eigentlichen Fragenkreises, der Erfassung des "typisch Offentlichen" einerseits und des "speziell Privaten" andererseits. ") Daher überrascht es nicht, daß auch der Terminus "öffentliches Gut" tragfähig ist sowohl für konkret umrissene Produktions-oder Dienstleistungsergebnisse (z.B. Fernsehsendungen, Orientierungs hilfe durch Leuchttürme) als auch für eher abstrakte und weit interpretierbare Politikfelder wie z.B. Umweltqualität ${ }^{2}$ ) oder Einkommensverteilung. ${ }^{3}$ )

Aus dieser großen Spannweite des Gutsbegriffs resultieren zusätzliche Probleme für die Aufgabenabgrenzung. Werden nämlich Güter funktional definiert (z.B. Erhöhung des Schutzes vor Einbruch), so ergibt sich daraus zwar der Vorteil der dimensionalen Identität von Gütern und Aufgaben. ${ }^{4)}$ Allerdings stößt nun die Identifikation der Güter als öffentliche oder private anhand gutstechnischer Eigenschaften auf Schwierigkeiten, weil - wie das Beispiel zeigt - die Erhöhung des Schutzes vor Einbruch sowohl durch eine Installation von Alarmanlagen (priva-

1) Zu Recht stellt deshalb Kirberger fest, daß den Bezeichnungen "Gut" oder "Aufgabe" im Grunde keine konstitutive Kraft zukommt; vgl. W. Kirberger, a.a.0., S. 55.

2) Vgl. Z.B. H. Siebert, Neuere Entwicklungen in der ökonomischen Analyse des Umweltschutzes, in: Umweltökonomik, hrsg. von H. Möller, Köniastein/ Ts. 1982 , S. $269 \mathrm{ff}$.

3) So insbesondere L. C. Thurow, The Income Distribution as a Pure Public Good, QJE Bd. 85 (1971), S. 328.

4) Zur Spannweite des Aufgabenbegriffs und zu den Dimensionen öffentlicher Aufgaben vgl. ersten Teil, Abschnitt I. 2. dieser Arbeit. 
tes Gut) als auch durch den Einsatz von Polizeistreifen (öffentliches Gut) zu erreichen ist. Werden statt dessen Güter als Produktions- und Dienstleistungsergebnisse aufgefaßt, so ist zwar die gutstechnische Zuordnung prinzipiell möglich, dafür besitzen die Güter aber im Hinblick auf die Aufgaben lediglich Mittelcharakter mit der Folge, daß der Nachweis bestimmter Giutereigenschaften noch nicht den - öffentlichen oder privaten Charakter einer Aufgabe determiniert.

1.5. Praktische Relevanz des Konzepts der öffentlichen Güter

Das Konzept der öffentlichen Güter bietet offenbar nicht das eindeutige Kriterium zur deskriptiven Abgrenzung, das man auf den ersten Blick erwarten konnte. Nichtrivalität allein ist zur Umschreibung öffentlicher Güter nicht hinreichend, zumal infolge des Auftretens von Kapazitätsgrenzen Rivalität entsteht. Die technische Nichtanwendbarkeit des Ausschlusses ist selten gegeben und die Umdeutung dieses Kriteriums zu einer ökonomischen Nichtanwendbarkeit des Ausschlußprinzips läßt eine exakte Grenzziehung nicht mehr zu.

Erfolgt eine strenge Auslegung der gutsspezifischen Eigenschaften, so stellen öffentliche Güter einen seltenen Ausnahmefall dar. Zwar können diese polaren Fälle dann ohne große Probleme zugeordnet werden, aber alle anderen Güter und Dienstleistungen werden dann undifferenziert als Mischgüter oder als private Güter ausgewiesen. Bei weniger strenger Auslegung der Kriterien wächst dagegen der Bereich öffentlicher Güter an, ohne daß aber noch klare Aussagen über ihren spezifischen Charakter getroffen werden könnten. Die Eigenschaft eines Gutes leistet keinen Beitrag mehr für eine eindeutige Aufgabenabgrenzung. Wie die schematische Darstellung in übersicht 9 zeigt, ergibt sich ein breiter Bereich von Mischformen zwischen die- 
sen beiden Polen, d.h. ein Kontinuum von Gütern, die sich in ihrem "offentlichkeitsgrad" 1) unterscheiden.

übersicht 9: Ausschlußprinzip und Rivalität im Konsum als Kriterien zur Unterscheidung öffentlicher und privater Güter - schematische Darstelluna -

\begin{tabular}{|c|c|c|c|}
\hline $\begin{array}{l}\text { Rivalität } \\
\text { Ausschluß- } \\
\text { prinzip }\end{array}$ & $\begin{array}{c}\text { Vollständige } \\
\text { Rivalität }\end{array}$ & $\begin{array}{c}\text { Partielle } \\
\text { (Nicht-)Rivalität }\end{array}$ & $\begin{array}{l}\text { Vollständige } \\
\text { Nichtrivalität }\end{array}$ \\
\hline anwendbar & $\begin{array}{c}\text { rein privates } \\
\text { Gut }\end{array}$ & Mischgut & Mischaut \\
\hline $\begin{array}{c}\text { nicht } \\
\text { anwendbar }\end{array}$ & Mischgut & Mischgut & $\begin{array}{c}\text { rein öfientliches } \\
\text { Gut }\end{array}$ \\
\hline
\end{tabular}

In der Literatur wird der Begriff der öffentlichen Güter häufig weiter gefaßt, um so eine praxisnähere Anwendung zu ermöglichen. Wir halten es für zweckmäßiger, den Begriff der öffentlichen Güter für jene Güter und Dienstleistungen zu reservieren, welche die beiden oben beschriebenen Kriterien aufweisen. Ist eine der beiden Eigenschaften nicht vorhanden, dann sollten zweckmäßigerweise auch andere Termini gewählt werden, z.B. Mischgüter (mixed goods), unreine

1) Vgl. dazu auch H. Bonus, offentliche Güter und der offentlichkeitsgrad von Gütern, $\mathrm{Zfg} \overline{\mathrm{S}} \mathrm{Bd} .136$ (1980), S. $50 \mathrm{ff}$. 
öffentliche Güter (impure public goods) oder abgrenzbare öffentliche Guiter (excludable public goods). 1)

Für eine deskriptive Aufgabenabgrenzung kommt also nur eine enge Auslegung der Kriterien in Betracht. Von dort ist es jedoch nur noch ein kurzer Schritt, der These von den durch spezifische Eigenschaften determinierten öffentlichen Gütern überhaupt jegliche praktische Relevanz abzusprechen. Offentliche Güter stellen nämlich nicht nur einen seltenen Polarfall dar, darüber hinaus ist es auch nicht möglich, sie bereits "aus ihrem Wesen" heraus zu definieren, d.h. die Eigenschaft eines Gutes existiert nicht "an sich" und damit unabhängig von Zeit und Raum, sondern zum einen verändert der technische Wandel die Eigenschaft der Güter, und zum anderen werden die Nichtanwendbarkeit des Ausschlußprinzips sowie die Nichtrivalität im Konsum vielfach erst durch institutionelle Voraussetzungen geschaffen.

1) Der Ausdruck "mixed goods" wird von Musgrave für jene Fälle verwendet, in denen private und öffentliche Gutseigenschaften kombiniert auftreten; vgl. R. A. Musgrave, Provision for Social Goods, a.a.0., S. $134 \mathrm{ff}$. "Impure pubTic goods" sind für Oakland durch das Auftreten partieller Rivalität gekennzeichnet; vgl. W. H. Oakland, a.a.0., S. $339 \mathrm{ff}$. Abarenzbare öffentliche Güter zeichnen sich dadurch aus, daß zwar das NichtRivalitätskriterium in hohem Maße erfüllt ist, es jedoch unschwer möglich ist, die zahlenden von den nicht zahlenden Konsumenten abzugrenzen; vgl. A. Endres, Neuere Entwicklungen in der Theorie der öffentlichen Guiter, ZfgS Bd. 137 (1981), S. 134. Einen überblick über verschiedene Mischguitermodelle und eine kritische Würdigung im Hinblick auf ihre finanztheoretische und - politische Relevanz aus funktionsanalytischer Sicht gibt W. Blaas, Zur Theorie der Mischgüter, in: Der öffentliche Sektor, Bd. 4 (1978), S. $63 \mathrm{ff}$. 


\section{Das Konzept der Koordinationsmechanismen}

Selbst wenn die spezifischen Eigenschaften eines Gutes oder einer Dienstleistung sich als Kriterium für eine Aufgabenabgrenzung als unzureichend erweisen, so hat die Erörterung doch bereits Hinweise auf eine weitere grundsätzliche Möglichkeit der Aufgabenabgrenzung gegeben. Von dem Charakter eines Gutes oder einer Dienstleistung wurde - unabhängig von der Trägerschaft auf die Art und Weise der Bereitstellung geschlossen. Dabei zeigte sich allerdings, daB die Zuordnung nach gutstechnischen Eigenschaften sich nicht mit der gegenwärtig in der Bundesrepublik Deutschland praktizierten Form der Bereitstellung deckt. Es scheint deshalb angebracht, die Gestaltung der Güterbereitstellung als eigenständiges Abgrenzungskriterium näher zu untersuchen. Damit sind konkret folgende Fragen zu beantworten:

- Welche typischen Allokations- und Verteilungsmechanismen sind in einer Volkswirtschaft unterscheidbar?

- Inwieweit können die in einer Volkswirtschaft bereitgestellten Güter und Dienstleistungen den unterscheidbaren Mechanismen eindeutig zugeordnet werden?

- Ergeben sich - vorausgesetzt, eine Zuordnung ist möglich aus einer solchermaßen vorgenommenen Klassifizierung Anhaltspunkte für eine exakte Trennungslinie zwischen öffentlichen und privaten Aufgaben?

Zunächst erscheint eine begriffliche Anmerkung notwendig. In der wirtschaftswissenschaftlichen Literatur stehen im Rahmen der Erörterung der Möglichkeiten gesamtwirtschaftlicher Steuerung vor allem Fragen der Allokation im Vordergrund des Interesses. Dies hat sich auch auf die verwendete Terminologie niedergeschlagen, denn vornehmlich wird in diesem Zusammenhang von "Allokationsmechanismen" gesprochen. 1) Da im folgenden aber auch uberlegun-

1) So z.B. E. Buschor, Theorie der Allokation der Staatsausgaben, Heerbrugg 1970, S. $4 \mathrm{ff}$.; L. Kohn, Probleme der Kollektivguiterallokation, Zürich 1973; E. Wille und M. Gläser, Staatsaufgaben bei Adam Smith eine Würdigung unter Allokationsaspekten, a.a.0., S. $43 \mathrm{f}$. 
gen einzubeziehen sind uiber die Art und Weise, wie das Eraebnis des Produktionsprozesses auf die Wirtschaftssubjekte verteilt wird, über welche Mechanismen mithin die personelle Verteiluna der Verfügungsmöglichkeiten über private und öffentliche Leistungen vonstatten geht, scheint es zweckmäßiger, den umfassenderen Begriff "Koordinationsmechanismen zu verwenden. 1)

\subsection{Iypologie der Koordinationsmechanismen}

Die geläufigste idealtypische Unterscheidung der volkswirtschaftlichen Koordinationsmechanismen ist die in Markt und Planung. Ist es in der Marktwirtschaft der Marktpreis, der für eine dezentrale Steuerung von Angebot und Nachfrage sorgt, so ist es in der Planwirtschaft der zentrale Plan, der die Lenkung des Produktions- und Verteilungsprozesses übernimmt. Im Mittelpunkt des ökonomischen Erkenntnisinteresses stand lange Zeit nahezu ausschließlich die Erforschung der Funktionsweise des Marktpreissystems. Der öffentlichen Planung kam deshalb eher der Charakter einer Restgröße zu. Inzwischen ist aber der öffentliche Sektor mehr in den Blickpunkt gerückt. Damit ist deutlich geworden, daß sich die dichotomische Betrachtungsweise in Markt und Planung als ungeeignet erweist, die Steuerungprinzipien einer Volkswirtschaft adäquat zu erfassen. ${ }^{2)}$ Vielmehr sind auch

1) Erst in jüngster Zeit wird des öfteren von Koordination, Koordinationsmethoden bzw. -mechanismen gesprochen; vgl. z.B. E. Bössmann, Volkswirtschaftliche Probleme der Transaktionskosten, ZfaS 138. Jg. (1982), S. $664 \mathrm{ff} . ; \mathrm{H}$. Grossekettler, Konzepte zur Beurteilung der Effizienz von Koordinationsmethoden, in: Jahrbuch für neue Politische Okonomie, 1. Jg. (1982), S. $213 \mathrm{ff} . ;$ H. H. Nachtkamp, Idealtypen von Fiskalsystemen. Ein Versuch, in: Finanzsysteme: Ideal-und Realtypen - Gesundhe itswesen und Hochschulbildung, hrsg. von K. Häuser, SchdVSocpol Bd. 135, Berl in 1983, S. 36 .

2) Die Zählebigkeit dieser dichotomischen Konzeption ist offenbar in erster Linie mit ihrer Griffigkeit für ungetrübte Schwarz-Weiß-Malereien in ordnungspolitischen Debatten über die vermeintliche uberlegenheit des einen oder anderen Wirtschaftssystems zu begründen; vgl. hierzu kritisch auch D. Lösch, Zur Ideologiekritik des traditionellen bipolaren Ordnungsdenkens, in: Hamburger Jahrbuch für Wirtschaftsund Gesellschaftspolitik, 20. Jg. (1975), S. $87 \mathrm{ff}$. 
im öffentlichen Bereich diverse Koordinationsmechanismen unterscheidbar und darüber hinaus Zwischenformen und Kombinationen marktijcher und nichtmarktlicher Koordination praktisch relevant, so daß die traditionellen Trennungslinien in zunehmendem Maße unscharfe Konturen erhalten.

Im folgenden soll eine über die bloße Zweiteilung hinausgehende Typologisierung gesamtwirtschaftlicher Lenkungsformen vorgenommen werden. Das konkrete typenbildende Merkmal bildet dabei die Art und Weise der Zuteilung knapper Mittel auf die Produktionsfaktoren bzw. auf die Wirtschaftssubjekte der Volkswirtschaft. Grundsätzlich können danach fünf fundamentale und wiederum idealtypische Koordinationsmechanismen unterschieden werden: Marktpreissystem, Windhundverfahren, Kontingentierung, Verlosung und Angebot bis zur Sättigung.

\subsubsection{Marktpreissystem}

Die Funktionsweise des Marktpreissystems muß hier nicht ausführlich beschrieben werden, aber zur Abgrenzung gegenüber den nachfolgenden Koordinationsmechanismen erscheint es anaebracht, die grundlegenden Charakteristika eines idealtypischen Marktpreissystems zu skizzieren.

Der Marktpreismechanismus beruht auf den Prinzipien des Tausches. Das Angebot einer Leistung hänat vom Zustandekommen einer Gegenleistung ab, Leistung und Gegenleistung erfolgen dabei Zug um Zug. Die hohen Transaktionskosten des reinen Tausches Ware gegen ware werden erheblich reduziert, indem mittels eines in Geldeinheiten ausgedrückten Preises die Bedinaungen bekanntgegeben werden, zu denen die Bereitschaft zum Tausch Ware gegen Geld besteht. Neben dieser Informationsfunktion besteht die wesentliche Leistung des Marktpreises jedoch in seiner Lenkungsfunktion. Der Marktpreis sorgt dafür, daß knappe Güter einer effizienten Verwendung zugeführt werden, d.h. er ist Indikator für die relative Knappheit der Güter und Dienstleistungen. Obersteigt die kaufkräftige Nachfrage nach einem Gut das zu einem bestimmten Preis bestehende Angebot, so steigt der Marktpreis 
zunächst. Ein steigender Marktpreis aber hat zur Folge, daß dieses Gut nunmehr von weniger Konsumenten nachgefragt wird. Es kommt zu einem Einpendlungsprozeß, der dann beendet ist, wenn Angebot und Nachfrage ubereinstimmen. Gleichzeitig werden durch den Anstieg des Marktpreises weitere nach Gewinn strebende Produzenten ermutigt, dieses Gut anzubieten. Eine das vorhandene Angebot übersteigende Nachfrage sorgt also nicht nur dafür, daß der Marktpreis steigt, sondern auch dafür, daß das Angebot ausgeweitet wird, d.h. die knappen Ressourcen kommen dort zum Einsatz, wo der Markt sie hinlenkt. Übersteigt das Angebot die Nachfrage, so gilt der umgekehrte Prozeß.

Das Marktpreissystem hat demnach also Markträumungsfunktion: Die gesamte kaufkräftige Nachfrage wird befriedigt. Die Betonung liegt dabei auf kaufkräftige Nachfrage, d.h. diejenigen Wirtschaftssubjekte, die nicht in der Lage sind, den aktue11 gültigen Marktpreis zu entrichten, werden vom Konsum der Güter ausgeschlossen.

\subsubsection{Windhundverfahren}

Windhundverfahren heißt ein Koordinationsmechanismus, wenn Güter und Dienstleistungen solange nach dem Prinzip "first come first served" bereitgestellt werden, bis das Angebot ausgeschöpft ist. Für diese Art der Zuteilung sind zwei Merkmale kennzeichnend:

Zum einen setzt die Anwendung des Windhundverfahrens eine das Angebot übersteigende Nachfrage voraus, und zum anderen wird die Lenkungsfunktion statt vom Preis nun von der Zeit übernommen ${ }^{1)}$, denn nur derjenige, der sich rechtzeitig in die Warte-

1) Wenngleich der Preis beim Windhundverfahren keine Lenkunasfunktion besitzt, so bedeutet dies freilich nicht, daß die Güterbereitstellung unentgeltiich zu erfolgen hat. Ex definitione muß der Preis nur niedriger sein als jener, der bei einer Marktpreiskoordination zustandekommt. Darüber hinaus können aber auch die Opportunitätskosten des Zeitaufwandes als Preis interpretiert werden; vgl. dazu E. Smolensky, T. N. Tidemann, D. Nichols, Waiting Time as a Congestion Charge, in: G. Mushkin (ed.), Public Prices for Public Products, Washinaton D.C. 1972, S. $95 \mathrm{ff}$.; E. Jonsson, On the Rationina of Public Products (Services) by Queuing vis-a-vis Rationina by Price, in: Public Finance, 36. Jg. (1981), S. $430 \mathrm{ff}$. 
schlange einreiht und bereit ist zu warten, besitzt die Gewähr, daß seine Nachfrage überhaupt berücksichtigt wird. 1) Praktische Anwendung findet das Windhundverfahren $z$. B. bei der Bereitstellung von Parkraum, bei der Verteilung von Taxikonzessionen, der Vergabe von Stellplätzen auf Jahrmärkten oder der Vermietung von Stadthallen. 2) Zudem erfolgt gelegentlich die Vergabe finanzieller Mittel nach dem Windhundverfahren (so z.B. Subventionen für die Sanierung von Altbauten im Rahmen des Konjunkturprogramms 1975). Mit dem Auftreten von Warteschlangen werden nicht selten unmittelbar Versorgungsiücken und Mißwirtschaft assoziiert. Gleichwohl handelt es sich hier lediglich um einen weiteren Typus gesamtwirtschaftlicher Koondination, der dem Marktpreissystem nicht bereits grundsätzlich unterlegen ist.

\subsubsection{Kontingentierung}

Knappe Güter und Dienstleistungen sowie monetäre Transfers können auf verschiedene Art und Weise kontingentiert werden. Eine Form der Kontingentierung ist die Verteilung knapper Güter über ein Bezugschein-system. So konnten z.B. in der Kriegsund Nachkriegszeit in Deutschland Nahrungsmittel nur über Lebensmittelkarten erworben werden. Die Karten begrenzten dabei die Menge, die der Einzelne von dem knappen Gut "Nahrungsmittel" in Anspruch nehmen konnte. Der AusschluB über den Preismechanismus war zu dieser zeit unerwinscht, vermutlich weil die Regulierung der übernachfrage über Preiserhöhungen die Zuteilung der vorhandenen Nahrungsmittel auf einige wenige kaufkräftige Nachfrager beschränkt hätte. Die Kontingentierung

1) Von Bedeutung sind also sowohl die Reaktionszeiten der Nachfrager (die wiederum ganz wesentlich von den Möglichkeiten der Informationsbeschaffung abhängen) als auch deren Bereitschaft, durch Warten in der Schlange Zeitverluste hinzunehmen.

2) Vgl. hierzu W. Berg, Die Verwaltung des Mangels. Verfassungsrechtliche Determinanten für Zuteilungskriterien bei knappen Ressourcen, in: Der Staat, 15. Jg. (1976), S. 16. 
vorhandener Güter und Dienstleistungen bietet sich demnach besonders als Koordinationsmechanismus für Mangelzeiten an: Niemand wird vom Konsum völlig ausgeschlossen, der Mangel wird statt dessen auf alle Konsumenten gleichmäßig verteilt. 1)

Allgemein kann von Kontingentierung immer dann gesprochen werden, wenn die Verwendung von knappen Ressourcen nach konkreten individuellen oder sachlichen Merkmalen gesteuert wird. Bei der Kontingentierung nach individuellen Merkmalen können u.a. bestimmte Qualifikationskriterien wie z.B. das Abitur bei der Vergabe von Studienplätzen ${ }^{2}$ oder persönliche Einkommensverhältnisse wie im Falle der Zuteilung von Sozialwohnungen herangezogen werden. Hingegen werden z.B. im Rahmen der finanziellen Förderung strukturschwacher Regionen sachliche Merkmale (Beschäftigungssituation, Industriebesatz, Höhe des Bruttoinlandsproduktes etc.) zugrundegelegt.

\subsubsection{Verlosung}

Knappe Güter können auch mit Hilfe des Losverfahrens zugeteilt werden. Nicht der Marktpreis und auch kein spezielles individuelles oder sachliches Merkmal lenken hier die Ressourcen, sondern allein der Zufall entscheidet über die Art der Verwendung und Verteilung. Jeder besitzt die gleiche Chance. Auch dieses Verfahren findet in der Praxis - wenn auch vergleichsweise selten - Anwendung oder wird als potentieller Koordinationsmechanismus diskutiert. Als Beispiele sind die Zuteilung von Grundstücken oder die Vergabe von Dauer-

1) Vgl. Ch. Tomuschat, Güterverteilung als rechtliches Problem, in: Der Staat, 12. Jg. (1973), S. 448; zu den theoretischen uberlegungen vgl. J. A. Tobin, A Survey of the Theory of Rationing, in: Econometrica, 20. Jg. (1952), S. $521 \mathrm{ff}$.

2) Die gegenwärtige Praxis der Studienplatzvergabe ist ein Beispiel für die teilweise Mehrfachanwendung der Kontingentierung. Zunächst bildet das bestandene Abitur selbst das Qualifikations- und damit gleichzeitig Auswahlkriterium, darüber hinaus erfolgt in den Numerus-Clausus-Fächern eine erneute Kontingentierung mittels der Abiturnote. 
parkplätzenzu nennen. Da die Kontingentierung der Studienplätze nach der Abiturnote wiederholt Gegenstand heftiger Kritik war, wurde bereits mehrfach der Obergang zum Losverfahren vorgeschlagen. 1)

\subsubsection{Angebot bis zur Sättigung}

Das Angebot bis zur Sättigung stellt das gebräuchliste nichtmarktliche Koordinationsverfahren dar. Unter Sättigung ist dabei freilich nicht die nur im Schlaraffenland mögliche vollständige Befriedigung sämtlicher Bedürfnisse zu verstehen, sondern vielmehr haben wir es auch weiterhin sehr wohl mit knappen Ressourcen zu tun. Allerdings wird aufgrund der technischen oder ökonomischen Eigenschaften öffentlicher Güter ${ }^{2}$ ) der Ausschluß eines Teils der Nachfrager als unzweckmäßig erachtet, oder es werden einige Güter und Dienstleistungen von den politischen Handlungsträgern als so bedeutend angesehen, daB sie über die sich am Markt bildende effektive Nachfrage hinaus ohne Anwendung eines speziellen Auswahlverfahrens für jedermann bereitgestel1t werden.

Zu unterscheiden ist zwischen einem Angebot bis zur Sättiqung mit und ohne Benutzerzwang, d.h. in manchen Fällen wird dem Konsumenten die freie Entscheidung darüber entzogen, ob und gegebenenfalls wieviel er von einem Gut nachfragen möchte. Dieser erzwungende Konsum kann entweder vornehmlich technisch bedingt (z.B. Verteidigung, Küstenschutz) oder aber primär das Resultat politisch für notwendig erachteter Versorgungsstandards sein (z.B. Schulpflicht, Impfpflicht). Anhand der übersicht 10 werden die vier unterscheidbaren Ausprägungen des Koordinations-

1) Siehe dazu die Nachweise bei Ch. Tomuschat, a.a.0., S. 446.

2) In diesem Zusammenhang spielt es keine Rolle, ob es die "wesensmäßigen" Eigenschaften bestimmter Güter oder eher die diese Eigenschaften erst begründenden institutionell getroffenen Vorkehrungen sind, die zu dem Verzicht auf Anwendung eines speziellen Auswahlverfahrens führen. 
mechanismus "Angebot bis zur Sättigung" verdeutlicht. Mit dem Auftreten eines Benutzungszwanges muß nicht notwendigerweise eine unentgeltliche Bereitstellung des Gutes einhergehen. Vielmehr können sogar an den Bereitstellungskosten orientierte Gebühren erhoben werden, wie dies beispielsweise bei der kommunalen Entsorgungsleistung "Müllabfuhr" der Fall ist.

Obersicht 10: Ausprägungen des Koordinationsmechanismus

"Angebot bis zur Sättigung"

\begin{tabular}{|c|c|c|}
\hline $\begin{array}{l}\text { Angebot } \\
\text { bis zur } \\
\text { Sättigung }\end{array}$ & $\begin{array}{l}\text { mit } \\
\text { Benutzungszwang }\end{array}$ & $\begin{array}{c}\text { ohne } \\
\text { Benutzungszwang }\end{array}$ \\
\hline $\begin{array}{c}\text { primär } \\
\text { technisch } \\
\text { bedingt } \\
\text { (öffentliche Güter) }\end{array}$ & $\begin{array}{l}\text { - Verteidigung } \\
\text { - Kuistenschutz }\end{array}$ & $\begin{array}{l}\text { - Fernsehsendung } \\
\text { - Straßenbenutzung }\end{array}$ \\
\hline $\begin{array}{c}\text { primär } \\
\text { politisch } \\
\text { gewollt } \\
\text { (meritorische Guiter) }\end{array}$ & $\begin{array}{l}\text { - Schulbesuch } \\
\text { - Impfung gegen } \\
\text { Epidemien }\end{array}$ & $\begin{array}{l}\text { - Hausarztbesuch } \\
\text { - Museumsbesuch }\end{array}$ \\
\hline
\end{tabular}

2.2. Alternative Typologien gesamtwirtschaftlicher Steuerungsformen

Bevor erörtert wird, welchen Beitrag die Koordinationsmechanismen zur Abgrenzung öffentlicher von privaten Aufgaben zu leisten vermögen, ist darauf hinzuweisen, daß verschiedentlich andere Typologien gesamtwirtschaftlicher Steuerungsformen verwendet werden. Verbreitet ist dabei insbesondere das Vorgehen, dem Marktpreissystem auf einer Ebene die Abstimmung und die Ver- 
handlung als gleichrangige Verfahren gegenüberzustellen. ${ }^{1}$ of fenbar wird dann aber eine andere Dimension gewählt, was auch in der verwendeten Terminologie zum Ausdruck kommt. FREY spricht $z$. B. von grundlegenden sozio-ökonomischen Entscheidungsmechanisme $^{2}$ ) , und LINDBLOM bezeichnet die von ihm beschriebenen Kategorien als politisch-ökonomische organisationsformen. ${ }^{3)}$ Die von uns charakterisierten fün fundamentalen Lenkungsprinzipien sind hingegen eher als technisch-ökonomische Koordinationsmechanismen zu verstehen.

Diese Aussage bedarf der näheren Erläuterung. Zur Abstimmung stehen vor allem die Programme der um Wählerstimmen konkurrierenden Parteien. Die Parteiprogramme unterscheiden sich im Idealfall durch gegensätzliche Vorstellungen u.a. über die Art und Weise der Bereitstellung von Gütern und Dienstleistungen. So präferiert beispielsweise die A-Partei die Bereitstellung des Gutes x über den Marktpreismechanismus, während sich die B-Partei dagegen für die Kontingentierung des Gutes $x$ nach einem individuellen oder sachlichen Merkmal einsetzt. Der Wähler stimmt also nicht nur über das Angebot der Güter ab, sondern ebenso über die zur Bereitstellung dieser Giuter anzuwendenden Koordinationsmechanismen. ${ }^{4}$ ) ahnliches gilt für die Verhandlung. Sie spielt zunächst primär als Konfliktmechanismus eine wesentliche Rolle (z.B. bei Tarifverhandlungen zwischen Gewerkschaf-

1) So z.B. L. Kohn, a.a.0., S. $107 \mathrm{ff}$.; ähnlich auch Frey, der zwischen Marktoder Preissystem, Demokratie, Hierarchie und Verhandlungssystem unterscheidet; B. S. Frey, Moderne politische Okonomie. Die Beziehunaen zwischen Wirtschaft und Politik, München und Zürich 1977, S. $24 \mathrm{ff} . ; \mathrm{vgl}$. dazu auch Lindblom, der die Kategorien Tausch, Autorität und Überreduna wählt; $\mathrm{Ch}$. E. Lindblom, Jenseits von Markt und Staat, Stuttgart 1980, S. $34 \mathrm{ff}$. Mit acht Koordinationsmechanismen stellt Shubik eine besonders breite Palette von Steuerungsverfahren gleichrangig nebeneinander: Marktpreissystem - Abstimmung - Auktion - Verhandlung - Autorität, Befehl oder Diktatur - Gewalt und Betrug - Tradition - Zufall; vgl. M. Shubik, On Different Methods for Allocating Resources, in: Kyklos, 23. Jg. (1970), S. $332 \mathrm{ff}$.

2) Vgl. B. S. Frey, a.a.0., S. 24.

3) Vgl. Ch. E. Lindblom, Jenseits von Markt und Staat, a.a.0., S. 34.

4) Allerdings wird dabei nicht über jedes Gut einzeln, sondern über aggregierte Güterbuindel abgestimmt. 
ten und Arbeitgeberorganisationen). Objekte der Verhandlung sind dann u.a. die Lohnhöhe, die Arbeitszeit und die Arbeitsbedingungen. Grundsätzlich kann aber auch über die Art der Bereitstellung knapper Giuter und Dienstleistungen verhandelt werden. Ange-

übersicht 11: Zusammenhang zwischen politisch-(sozio-)ökonomischen Entscheidungs- und technisch-ökonomischen Koordinationsmechanismen

Verfahren zur Herbe iführung gesellschaftlicher Entscheidungen

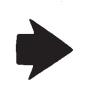
politisch-(sozio-)ökonomische Entscheidungsmechanismen

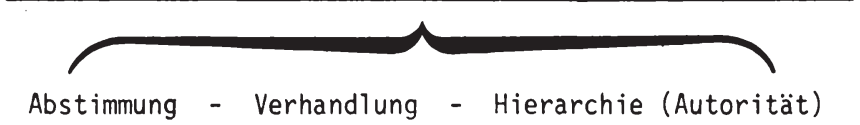

Abstimmung - Verhandlung - Hierarchie (Autorität)

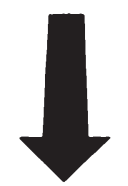

Marktpreissystem - Windhundverfahren - Verlosung

- Kontingentierung - Angebot bis zur Sättigung

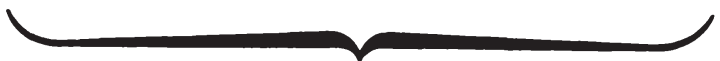

Verfahren zur

Steuerung

knapper

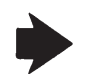

technisch-ökonomische Koordinationsmechanismen

nommen, die Anwohner einer Straße könnten selbst untereinander aushandeln, ob die Straße für jeden Verkehr offengehalten oder als Fußgängerzone für den Autoverkehr gesperrt werden soll. Gegenstand der Verhandlung sind damit alternative Koordinationsmechanismen: Das Gut Straße wird im ersten Fall bis zur Sätti- 
gung angeboten, da jedermann die Straße im Rahmen seiner Präferenzen benutzen kann. Dagegen wird im zweiten Fall das Angebot nach einem spezifischen Merkmal (Fußgänger) kontingentiert.

Aus übersicht 11 wird der Zusammenhang zwischen den zur Herbeiführung gesellschaftlicher Entscheidungen eingesetzten politisch-bzw. sozio-ökonomischen Mechanismen einerseits und den zur Steuerung knapper Ressourcen dienenden technisch-ökonomischen Mechanismen andererseits ersichtlich. Erstere sind allgemeinerer Art und letzteren zeitlich vorgeschaltet. Die Tatsache, daB die Bereitstellung von Gütern und Dienstleistunqen über den ProzeB der Abstimmung erfolgt oder als Ergebnis von Verhandlungen zustandekommt, läßt offen, ob die Steuerung dieser Güter und Dienste beispielsweise über eine Form der Kontingentierung oder mit $\mathrm{Hilfe}$ des Marktpreises vorgenommen wird. Nicht die Abstimmung und Verhandlung als solche sind aus ökonomischer Sicht als Koordinationsmechanismen zu betrachten, vielmehr wird erst im Rahmen der Abstimmung bzw. Verhandlung über die Anwendung alternativer Koordinationsmechanismen entschieden.

\subsection{Praktische Relevanz des Konzepts der Koordinations-} mechanismen

Zu prüfen ist nunmehr, inwieweit die Bereitstellung von Gütern und Diensten den einzelnen Koordinationsmechanismen eindeutig zugeordnet werden kann und ob die Art der gesamtwirtschaftlichen Koordination überhaupt ein geeignetes Kriterium bietet, das eine Trennung zwischen öffentlichen und privaten Aufgaben sinnvoll erlaubt.

Die Obersicht 12 verdeutlicht, daß in der Mehrzahl der Fälle eine Zuordnung der Güterbereitstellung zu einem der fünf fundamentalen Koordinationsmechanismen ohne große Probleme möglich ist. Dabei zeigt sich, daß in der Bundesrepublik gegenwärtig vor allem drei Mechanismen eine herausragende Rolle 
Übersicht 12: Koordinationsmechanismen als Kriterium zur Abarenzung öffentlicher von privater Güterbereitstellung

- Beispiele -

\begin{tabular}{|c|c|c|c|c|c|c|}
\hline \multirow[b]{2}{*}{$\begin{array}{l}\text { Guiter und } \\
\text { Dienstleistungen }\end{array}$} & \multirow{2}{*}{$\begin{array}{l}\text { Markt- } \\
\text { preis- } \\
\text { system }\end{array}$} & \multirow{2}{*}{$\begin{array}{l}\text { Wind- } \\
\text { hund- } \\
\text { verfah- } \\
\text { ren }\end{array}$} & \multirow{2}{*}{$\begin{array}{l}\text { Verlo- } \\
\text { sung }\end{array}$} & \multirow{2}{*}{$\begin{array}{l}\text { Kont ingen- } \\
\text { tierung }\end{array}$} & \multicolumn{2}{|c|}{$\begin{array}{c}\text { Angebot bis zur } \\
\text { Sättiquno }\end{array}$} \\
\hline & & & & & $\begin{array}{l}\text { ohne } \\
\text { Benutzungs- } \\
\text { zwang }\end{array}$ & $\begin{array}{l}\text { mit } \\
\text { Benutzungs- } \\
\text { zwang }\end{array}$ \\
\hline $\begin{array}{l}\text { SICHERHEIT/ORDNUNG } \\
\text { nationale Verteidigung } \\
\text { Verbrechensbekämpfung } \\
\text { individueller Objekt-/ } \\
\text { rersonenschutz } \\
\text { Verkehrscegelung } \\
\text { PaBausstellung } \\
\text { Feuerschutz } \\
\text { Kuistenschutz } \\
\text { BILUUNG }\end{array}$ & + & & & + & + & $\begin{array}{l}+ \\
+ \\
+ \\
+ \\
+\end{array}$ \\
\hline $\begin{array}{l}\text { BILUUNG } \\
\text { allg. Schulbildung } \\
\text { Hochschulbildung } \\
\text { betriebliche Ausbildung } \\
\text { Weiterbildung (z.B. VHS })\end{array}$ & & $\begin{array}{l}+ \\
+\end{array}$ & + & $\stackrel{+}{+}$ & + & + \\
\hline $\begin{array}{l}\text { GESUNOHEIT/SOZIALES } \\
\text { Hausarzt } \\
\text { Krankenhaus } \\
\text { Schutzimpfung gegen } \\
\text { Epidemien } \\
\text { Reinenuntersuchung } \\
\text { Al tersheim } \\
\text { Sozialwohnung }\end{array}$ & + & $\stackrel{+}{+}$ & & $\begin{array}{l}+ \\
+ \\
+ \\
+\end{array}$ & + & + \\
\hline $\begin{array}{l}\text { KUL TUR/UNTERHALTUNG/ } \\
\text { ERHOLUNG/SPORT } \\
\text { Museum } \\
\text { Bucherei } \\
\text { Kino/Theater } \\
\text { Fernsehsendung } \\
\text { Zeitschriften } \\
\text { Parkanlage } \\
\text { Sportveranstaltung }\end{array}$ & $\begin{array}{l}+ \\
+ \\
+\end{array}$ & + & & & $\begin{array}{l}+ \\
+ \\
+ \\
+ \\
+ \\
+\end{array}$ & \\
\hline $\begin{array}{l}\text { INFRASTRUKTUR/VERKEHR } \\
\text { Straben } \\
\text { FuBgängerzone } \\
\text { parkplätze } \\
\text { Eisenbahn } \\
\text { Stronl-, Gas-, Wasser- } \\
\text { versorgung } \\
\text { Kanal isation }\end{array}$ & $\begin{array}{l}(+) \\
(+)\end{array}$ & + & & + & $\begin{array}{l}+ \\
(+) \\
(+)\end{array}$ & + \\
\hline $\begin{array}{l}\text { SONSTIGE GOTER- UND } \\
\text { OIENSTLEISTUNGEN } \\
\text { Lebensmittel } \\
\text { Autos } \\
\text { Wohnungseinrichtungen } \\
\text { Reisen }\end{array}$ & $\begin{array}{l}+ \\
+ \\
+ \\
+\end{array}$ & & & & & \\
\hline
\end{tabular}


spielen. Neben dem Marktpreissystem sind dies die Kontingentierung und das Angebot bis zur Sättigung, während das Windhundverfahren und die Verlosung seltener Anwendung finden. Allerdings sind in einigen Fällen auch Kombinationen mehrerer Koordinationsmechanismen oder Zwischenformen zu konstatieren. So kann z.B. die Vergabe eines Altersheimplatzes von konkreten individuellen Voraussetzungen (z.B. Einkommensverhältnissen) abhängig gemacht werden, d.h. zunächst wird die kontingentierung angewandt. Unter denjenigen, die die gesetzten Voraussetzungen erfiullen, wird anschließend eine Zuteilung nach dem Windhundverfahren vorgenommen. Zwischenformen treten u.a. bei einigen gegen Gebühren bereitgestellten Versorgungs- (z.B. Strom, Gas, Wasser) oder Verkehrsleistungen (z,B. Eisenbahn) auf. Komponenten des Marktpreissystems werden hier mit Elementen einer Steuerung des Angebots bis zur Sättigung verknüpft. Marktähnliche Beziehungen zwischen Anbietern und Nachfragern ergeben sich aus der Möglichkeit des Ausschlusses zahlungsunwilliger Wirtschaftssubjekte, d.h. insofern erfolgt eine simulierung privatwirtschaftlicher Tauschvorgänge nach den Prinzipien des do ut des. Indes mangelt es an einem wesentlichen Charakteristikum des Marktpreissystems: Die Höhe der Gebühren orientiert sich im Gegensatz zum Marktpreis nicht an Angebot und Nachfrage, sondern wird durch eine politische Entscheidung festgesetzt. Elemente des Angebots bis zur Sättigung ergeben sich z.B. aus der Anschluß- und Betriebspflicht (Versorgungszwang) der Anbieter. 1) Da die Nachfrage nach Versorgungs- und Verkehrsleistungen gleichwohl von der Höhe der Gebihren abhängig ist, geht freilich mit steigenden Gebihren der spezifische Charakter des Angebots bis zur sättigung zunehmend verloren.

Trotz der auftretenden Kombinations- und Zwischenformen gelingt die Zuordnung der Güter und Dienste zu den einzelnen Koordina-

1) Bei der Strom-, Gas- und Wasserversorgung besteht auch auf der Nachfrageseite ein Anschlußzwang, während die tatsächliche Inanspruchnahme der freien Entscheidung jedes einzelnen Konsumenten überlassen bleibt. Die Kanalisation ist dagegen mit einem Benutzungszwang (Anschluß- und Verbrauchszwang) verbunden. 
tionsmechanismen wesentlich besser als die Identifikation privater und öffentlicher Güter anhand gutstechnischer Eigenschaften. Dennoch treten auch hier zum Teil ähnliche Schwieriakeiten auf, wie sie bereits im Zusammenhang mit dem Konzept der öffentlichen Güter festzustellen waren. Die Form der Güterkoordination determiniert noch nicht in hinreichender Weise den - privaten oder öffentlichen - Charakter einer Aufgabe. Die unterschiedliche Dimension von Gütern (Produktebene) und Aufgaben (Funktionsebene) erweist sich wiederum als Barriere für die Abarenzuna privater und öffentlicher Bereiche, so daß letztlich auch das Kriterium "Koordinationsmechanismen" kaum mehr als ein Hilfselement zur Erschließung des "typisch offentlichen" einerseits und des "eigentlich Privaten" andererseits sein kann.

Abgesehen von diesen Schwierigkeiten besitzt freilich das Abgrenzungskriterium "Koordinationsmechanismen" gegenüber den bisher diskutierten Kriterien eine Reihe von Vorteilen:

- Es wird auf ein formales Kriterium abgestellt und damit von vornherein auf den - wie die funktionale Betrachtungsweise gezeigt hat: vergeblichen - Versuch verzichtet, aus der vermeintlichen Zwecksetzung wirtschaftlicher Aktivität die "offentlichkeit" einer Aufgabe materiell abzuleiten.

- Die "Offentlichkeit" einer Aufgabe wird unabhängig von dem institutionellen Gesichtspunkt der Trägerschaft bestimmt. Damit wird der Tatsache Rechnung getragen, daß sich der öffentliche Sektor genauso des Marktpreissystems bedient (z.B. dort, wo der Staat als Anbieter mit privaten Unternehmen am Markt konkurriert) wie auch im privaten Sektor häufig andere Koordinationsformen als der Marktpreismechanismus Anwendung finden (z.B. Einrichtung einer 
Kindertagesstätte für berufstätige Familien durch Selbstorganisation). 1)

- Diese Form der Abgrenzung greift nicht so kurz wie die Charakterisierung öffentlicher Güter und Dienstleistungen mit Hilfe gutstechnischer Eigenschaften. Damit besteht hier die Möglichkeit einer Zuordnung über den engen Bereich einiger polarer Fälle hinaus.

\section{Ergebnisse}

Die Diskussion funktionaler, institutioneller und technischökonomischer Kriterien hat gezeigt, daß diese im Hinblick auf ihre Tauglichkeit zur deskriptiven Abgrenzung öffentlicher und privater Aufgaben recht unterschiedlich zu bewerten sind. Zur Beurteilung der Kriterien wurden dabei vor allem zwei Maßstäbe angelegt: Eindeutigkeit ihrer öffentlichen bzw. privaten Ausprägungen und die Zuordnungsmöglichkeit der Aufgaben zu diesen Ausprägungen (siehe übersicht 13).

Dem Versuch, öffentliche Aufgaben funktional gemäß ihrer Zwecksetzung zu bestimmen, liegt die Vorstellung zugrunde, öffentliche Aufgaben seien an gesellschaftlichen Zielen orientiert, sie erfolgten damit im Dienste des Gemeinwohls, während private Aufgaben vornehmlich durch die Erfüllung von Einzelinteressen gekennzeichnet seien und somit der individuellen Nutzenmaximierung dienten. Obschon von beträchtlichem ökonomischen Inter-

1) Damit wird auch erneut die rein institutionelle Argumentationsweise der Privatisierungsdebatte offensichtlich. Gewöhnlich wird von Privatisierung der Müllabfuhr, des offentlichen Personennahverkehrs etc. gesprochen, wenn diese Aufgaben - überwiegend sogar nur bestimmte Teilfunktionen der Aufgaben - privaten Trägern übertragen werden. Wird die Ausgestaltung des Koordinationsmechanismus als entscheidendes Abgrenzungskriterium herangezogen, so könnte sinnvollerweise von einer Privatisierung immer nur dann gesprochen werden, wenn eine bestimmte Tätigkeit vollständig der Funktionsweise des Marktpreissystems überlassen wird. Weder im Hinblick auf die Müllabfuhr noch hinsichtlich des offentlichen Personennahverkehrs wird jedoch daran gedacht, die Bereitstellung dieser Güter einer Marktpreissteuerung zu überlassen. 
esse, leidet dieses Vorgehen aber bereits erheblich unter dem Mangel, daß es nicht möglich ist, das Gemeinwohl ex ante objektiv zu bestimmen und demzufolge auch eine klare materielle Trennung zwischen Aktivitäten, die dem Gemeinwohl dienen, und Handlungen, die aus dem Eigennutzmotiv hervorgehen, nicht durchführbar ist. Folglich kann eine Identifikation privater und öffentlicher Aufgaben von der Anwendung funktionaler Abgrenzunaskriterien nicht erwartet werden.

Obersicht 13: Eine grobe Matrix zur Beurteilung der Abgrenzungskriterien

\begin{tabular}{|c|c|c|c|c|c|}
\hline \multirow[b]{2}{*}{$\begin{array}{c}\text { Beurteilungs } \\
\text { maBstäbe }\end{array}$} & funktionale & \multicolumn{2}{|c|}{ institutionelle } & \multicolumn{2}{|c|}{ technisch-ökonomische } \\
\hline & $\begin{array}{c}\text { Geme inwohl vs. } \\
\text { Eigennutz }\end{array}$ & $\begin{array}{l}\text { Rechts - } \\
\text { formen }\end{array}$ & $\begin{array}{c}\text { Eigentums - } \\
\text { verhältnisse }\end{array}$ & $\begin{array}{l}\text { Eigen- } \\
\text { schaften }\end{array}$ & $\begin{array}{l}\text { Koordinations } \\
\text { mechanismen }\end{array}$ \\
\hline Eindeutigkeit & - & + & 0 & 0 & + \\
\hline $\begin{array}{l}\text { Zuordnungs- } \\
\text { möglichkeit }\end{array}$ & - & - & - & - & 0 \\
\hline
\end{tabular}

+ weitgehend erfüllt

- mit erheblichen [inschränkungen erfillt

- weitgehend nicht erfült

Kaum günstiger zu beurteilen sind Versuche einer institutionellen Aufgabenabgrenzung. Zwar gelingt es zunächst, anhand der Rechtsformen und der Eigentumsverhältnisse in der Tendenz eine Trennung öffentlicher und privater Aufgabenträger vorzunehmen, hingegen ist eine eindeutige Zuordnung der Aufgaben zu diesen Trägern in den meisten Fällen nicht möglich, da öffentliche Aufgaben sowohl von öffentlichen als auch von privaten Trägern durchgeführt werden, ebenso wie private Aufgaben nicht nur von 
privaten, sondern auch von öffentlichen Trägern wahrgenommen werden. Darüber hinaus ist die ökonomische Relevanz institutioneller Abgrenzungskriterien eher gering zu veranschlagen.

Eine differenzierte Bewertung ist bezüglich der technisch-ökonomischen Abgrenzungskriterien erforderlich. Aufgrund methodischer Schwierigkeiten gelingt eine Unterscheidung öffentlicher und privater Güter mit Hilfe gutsspezifischer Eigenschaften nur mit erheblichen Einschränkungen. Vor allem ist die praktische Bedeutung dieses Konzepts gering, weil mit ihm allenfalls einige wenige polare Fälle erfaßt werden können, während die Zahl der nicht eindeutig zurechenbaren Mischformen bei weitem überwiegt. Schließlich: Da aus der Eigenschaft eines Gutes noch nicht auf den Charakter einer Aufgabe geschlossen werden kann, leistet auch das Konzept der öffentlichen Güter keinen nennenswerten Beitrag zur Aufgabenabgrenzung. Obschon die unterschiedliche Dimension von Gütern und Aufgaben auch im Hinblick auf das Kriterium "Koordinationsmechanismen" die Aufgabenabgrenzung erschwert, besitzt es immerhin den Vorteil, daß die Güter und Dienstleistungen den einzelnen Koordinationsmechanismen überwiegend zugeordnet werden können. Als weitere Vorzüge sind die Anknüpfung an formale Gegebenheiten sowie die von der Trägerschaft unabhängige Zuordnung zu werten.

Insgesamt ist aus der Würdigung der verschiedenen Abgrenzungsversuche ein eher nüchternes Resümee zu ziehen: Bei den öffentlichen Aufgaben handelt es sich um eine äußerst amorphe Masse, die sich dem Betrachter immer nur ausschnittweise erschlieBt, ihm aber niemals zugleich einen umfassenden Blick gewährt. Aus diesem Grunde ist auch eine deskriptive Abgrenzung öffentlicher von privaten Aufgaben nur partiell mit Hilfe verschiedener Kriterien möglich, die sich aus dem spezifischen Erkenntnisinteresse des jeweiligen Betrachters ergeben. 
Warum wurde der Abgrenzungsfrage dieser breite Raum gewidmet, wenn damit zu rechnen war, daß nur ein vergleichsweise karges Ergebnis präsentiert werden könnte? Mindestens zwei Gründe rechtfertigen den Aufwand:

- Der Versuch, mit Hilfe verschiedener Kriterien eine Abgrenzung öffentlicher von privaten Aufgaben vorzunehmen, wird nicht nur zu einer Dokumentation der Komplexität öffentlicher Aufgaben, sondern er leistet gleichzeitig einen Beitrag zur terminologischen Klarstellung und zur Systematisierung der in der Wissenschaft, vor allem aber in der Politik mit uneinheitlichen Begriffen und aus unklaren Begründungszusammenhängen geführten Diskussion über die "Entstaatlichung" bzw. "Privatisierung" öffentlicher Aufgaben.

- Die Versuche einer deskriptiven Aufgabenabgrenzung erleichtern den Zugang zur nachfolgenden normativen Analyse, insbesondere ermöglichen sie die Vorstrukturierung der Problembereiche und Fragestellungen, "auf die es ankommt". Die Erörterung der Abgrenzungskriterien hat gezeigt, daß öffentliche Aufgaben sowohl von öffentlichen als auch von privaten Institutionen wahrgenommen werden, der Stat sich zur Aufgabenerfuillung eines breiten Fächers alternativer Instrumente bedient und zur Güterversorgung ganz unterschiedliche Koordinationsmechanismen zur Verfügung stehen. Vor diesem Hintergrund sind im folgenden die in der wirtschaftswissenschaftlichen Literatur diskutierten normativen Kriterien einer kritischen oberprüfung zu unterziehen und, soweit erforderlich, ökonomische kriterien für eine zweckmäßige Aufgabenverteilung in der Marktwirtschaft, d.h. Kriterien für ein ökonomisch rationales Zusammenspiel dieser Institutionen, Instrumente und Koordinationsmechanismen, zu entwickeln. 


\section{NORMATIVE KRITERIEN FÜR EINE AUFGABENVERTEILUNG IN DER MARKTWIRTSCHAFT}

\section{Zur konzeptionellen und methodologischen Trennung von Allokation und Distribution}

Erst aus der Knappheit wirtschaftlicher Ressourcen erwächst die Notwendigkeit zum ökonomischen Handeln. Die Tatsache, daß Ressourcen in jeder Volkswirtschaft knapp sind, läßt das knappheitsproblem zum zentralen Gegenstand der ökonomie überhaupt werden. In jeder Gesellschaft - unabhängig von der Ausgestaltung des Wirtschaftssystems - müssen deshalb drei arundlegende wirtschaftspolitische Entscheidungen getroffen werden, und zwar

1. Entscheidungen darüber, welche Guiter und Dienstleistungen bereitgestellt werden sollen;

2. Entscheidungen uiber die Art und Weise der Bereitstellung von Gütern und Dienstleistungen, genauer, mit Hilfe welcher Institutionen, durch den Einsatz welcher Instrumente und mittels welcher Koordinationsmechanismen eine möglichst effiziente Nutzung der knappen Ressourcen angestrebt werden soll;

3. Entscheidungen darüber, wie das Angebot der Güter und Dienstleistungen auf die Individuen der Gesellschaft verteilt werden soll.

Die ersten beiden Entscheidungsbereiche können der Allokation, der dritte Bereich kann der Distribution zugeordnet werden. 1)

1) In der Finanzwissenschaft ist die auf Musgrave zurückgehende Dreiteilung der Budgetpolitik in eine Allokations-, Distributions- und Stabilisierungsfunktion verbreitet; vgl. R. A. Musgrave, The Theory of Public Finance, a.a.0., S. $5 \mathrm{ff}$. Da Marktwirtschaften zu Schwankungen des Preisniveaus und der Beschäftigung neigen, sind neben Allokations- und Distributionsentscheidungen auch Stabilisierungsentscheidungen mit dem Ziel zu treffen, diese Schwankungen in Grenzen zu halten. Gleichwohl kann das Stabilisierungsproblem aber als ein Problem der zeitlichen Allokation aufgefaßt werden. 
De facto wird freilich mit jeder Allokationsentscheidung stets zugleich auch eine Entscheidung über die Distribution getroffen. Die personelle Einkommensverteilung hängt ab von der Struktur der Faktoreinkommen am Markt, die sich als Resultat allokativer Maßnahmen ergibt. Die Allokationsergebnisse hängen ihrerseits von der jeweiligen effektiven Nachfrage ab, die wiederum durch distributive Entscheidungen bestimmt wird.

Der Hinweis, daß eine ökonomisch rationale Wirtschaftspolitik die simultane Lösung der Allokations- und Distributionsprobleme erfordert ${ }^{1}$, birgt jedoch im Grunde nichts anderes als die banale Feststellung, daß in einer Volkswirtschaft letztlich alles wirtschaftliche Handeln interdependent ist. Die Durchführung ökonomischer Analysen macht deshalb rein gedankliche Trennungen unumgänglich, denn sie erlauben es, an verschiedenen Stellen Pflöcke in die Untersuchung einzuschlagen, die dem Analytiker orientierungsmarken und Anbindungspunkte zugleich bieten.

Die Möglichkeit der gedanklichen Trennung wird recht weitgehend genutzt, indem die Untersuchung ausschließlich auf allokative Gesichtspunkte begrenzt bleibt. Diese Beschränkung erfolat freilich nicht deshalb, weil distributive Fragestellungen als weniger bedeutsam ${ }^{2}$ ) oder für den Wirtschaftswissenschaftler als prinzipiell unzugänglich ${ }^{3}$ ) erachtet werden. Die Konkretisierung der Leitlinien der Verteilungsgerechtigkeit bereitet jedoch im Vergleich zur Formulierung allokativer Zielsetzungen weitaus

1) Auch Musgrave weist ausdrücklich auf die Interdependenzen zwischen den Funktionen hin; vgl. R. A. Musgrave, The Theory of Public Finance, a.a.0., S. $20 \mathrm{ff}$. sowie S. $28 \mathrm{ff}$.

2) So sieht z.B. Walter Eucken auch heute noch in dem Verteilungsproblem das primäre wirtschaftliche Problem für die meisten Menschen; vgl. W. Eucken, Grundsätze der Wirtschaftspolitik, 5. Auf1., Bern und Tübingen 1975, S. 12 .

3) Little bemerkt hierzu, "that many economists wrongly believe that they are being perfectly scientific and objective if they say 'If price is not equal marginal cost, welfare could be increased' and unscientific if they say 'Welfare could be increased by redistributing wealth in such and such way'." I. M. D. Little, A Critique of Welfare Economics, 2. Auf1., Oxford 1957 , S. 267, Fußn. 2. 
größere Probleme. 1) Da außerdem die distributiven Kriterien für eine Aufgabenverteilung aus einem anderen Bezugssystem als die allokativen kriterien abgeleitet werden muissen ${ }^{2}$, würde die Berücksichtigung verteilungstheoretischer und -politischer Erwägungen den Rahmen der Arbeit sprengen.

Die Funktionentrennung MUSGRAVES dient einerseits einem konzeptionellen Zweck, daruber hinaus bildet sie aber auch die Grundlage für die im Rahmen der Wohlfahrtsökonomik entwickelten normativen Theorie der Staatswirtschaft. Sie erfüllt somit auch einen methodologischen Zweck.

BUCHANAN hat im Hinblick auf die methodologische Zwecksetzuna die Vorgehensweise MUSGRAVES mehrfach kritisiert, da sie unvermeidlich zu logischen Schwierigkeiten führe. ${ }^{3)}$ Es sei methodologisch inkonsequent, Kriterien für eine effiziente Allokation der Ressourcen isoliert gemäß den individuellen Präferenzen der Wirtschaftssubjekte zu bestimmen (Anerkennuna der Konsumentensouveränität), die Normen für die Verteilung hingegen aufgrund externer, überindividueller Wertungen zu gewinnen (Eingriff in die Konsumentensouveränität). ${ }^{4}$ Er spricht sich deshalb dafür aus, überhaupt auf die Einführung nichtindividueller Normen zu verzichten, weil sonst in der offentlichkeit fälschlicherweise der Eindruck entstehen könnte, "that economists have a hot line to God". 5)

1) Krause-Junk stellt fest, daß die Verteilungsprinzipien zwar einfach zu formulieren, aber schwierig zu interpretieren seien, denn "obwohl sie sozusagen die Eckpunkte des Feldes abstecken, in dem jeder einzelne seine Gerechtigkeitsvorstellungen angesiedelt hat, und ihr Sinngehalt in jedem Fall eingängig erscheint, erlauben sie als Instrument in der Hand eines virtuosen Sophisten die Rechtfertigung nahezu jeder denkbaren Verteilung." G. Krause-Junk, Zur Theorie des distributiven Marktversagens, in: offentTiche Finanzwirtschaft und Verteilung II, hrsg. von W. Aibers, SchdVSocpol, N.F., Bd. 75/II, Berlin 1974, S. 36.

2) Vgl. etwa den Ansatz von G. Krause-Junk, Zur Theorie des distributiven Marktversagens, a.a.0., S. $33 \mathrm{ff}$.

3) Vgl. insbesondere J. M. Buchanan, The Theory of Public Finance, SEJ Vol. 26 (1960), S. 234 ff. Sowie ders., What Kind of Redistribution Do We Want?, in: Economica, Vol. 25 (1968), S. $188 \mathrm{f}$.

4) Vgl. J. M. Buchanan, What Kind of Redistribution Do We Want?, a.a.0., S. 188 .

5) Ebenda, S. 189. 
Verschiedentlich ist deshalb der Versuch unternommen worden, diesen Vorwurf der Inkonsequenz zu heilen, indem auch für den Bereich der Distribution auf die individuellen Präferenzen der Wirtschaftssubjekte zurückgegriffen wird. ") Solche Versuche können allerdings lediglich Teilbereiche der Verteilungsproblematik erfassen. 2)

Da MUSGRAVE die Notwendigkeit von Eingriffen in die Konsumentensouveränität auch im Hinblick auf allokative Zielsetzungen ausdrücklich anerkennt ${ }^{3)}$, rückt er vom extrem individualistischen Standpunkt ab. Deshalb verliert BUCHANANS Vorwurf der methodologischen Inkonsequenz zumindest hier seine Berechtigung. Ein solches Vorgehen ist zwar formal wenig elegant, und MUSGRAVE selbst sieht darin sogar eine "heikle Angelegenheit"4). Die Berücksichtigung des Führungsverhaltens politischer Handlungsträger dürfte aber im Vergleich zur Fiktion der Konsumentensouveränität den Ausgangspunkt für wesentlich realistischere Interpretationen der Entscheidungsprozesse in der Demokratie bilden.

\section{Theoretischer Rahmen der allokativen Analyse}

Um rational darüber entscheiden zu können, welche Aufgabenverteilung zwischen dem privaten und dem öffentlichen Sektor einer Volkswirtschaft ökonomisch zweckmäßig ist, müssen die Entscheidungen anhand zuvor entwickelter Kriterien erfolgen. Diese Kriterien sind aber nur dann sinnvoll zu erarbeiten, wenn sie einen gemeinsamen Bezugspunkt aufweisen. Es muß

1) So vor allem H. M. Hochman und J. D. Rodgers, Pareto Optimal Redistribution, AER Bd. 59 (1969), S. 542 ff.

2) $\mathrm{Vgl}$. dazu etwa E. Knappe, Einkommensumverteilung in der Demokratie, Freiburg i. Br. 1980, S. $279 \mathrm{ff}$.

3) $\mathrm{Vgl}$. dazu seine Ausführungen zu den meritorischen Gütern; R. A. Musgrave, The Theory of Public Finance, a.a.0., S. $13 \mathrm{f}$.

4) Ebenda, S. 14. 
also zunächst ein Referenzsystem entworfen werden, aus dem die ökonomischen Kriterien für eine Aufgabenverteilung abgeleitet werden können. Grundsätzlich sind eine unendliche Zahl verschiedener Referenzsysteme denkbar. Nachfolgend werden zwei Referenzsysteme eingehender behandelt, die in unterschiedlichem Maße Eingang in die ökonomische Theorie einerseits und in die wirtschaftspolitische Praxis andererseits gefunden haben: die paretianische (neuere) Wohlfahrtsökonomie und die Analyse gesellschaftlicher Ziele.

1. Das wohlfahrtsökonomische Referenzsystem

1.1. Skizzierung des wohlfahrtsökonomischen Argumentationsgerüstes

In der ökonomischen Theorie bildet ganz überwiegend das wohlfahrtsökonomische Modell des allgemeinen Konkurrenzgleichgewichts den Ausgangspunkt für Oberlegungen über die "richtige" Aufgabenverteilung zwischen dem privaten und dem öffentlichen Sektor der Volkswirtschaft. Es muB hier darauf verzichtet werden, Entwicklung und Stand der Wohlfahrtsökonomie in allen Einzelheiten nachzuzeichnen ${ }^{1)}$, lediglich ihr Argumentationsgerust soll in aller Kürze vorgestellt werden.

Als Wohlfahrtsökonomik (welfare economics) wird der Zweig der Wirtschaftswissenschaft bezeichnet, der sich mit der Frage beschäftigt, wie auf Grundlage allgemein akzeptierter Wertvorstellungen und unter Einhaltung ganz konkreter Bedingungen die knappen Ressourcen optimal genutzt werden können und so das Ziel eines maximalen Wohlstandes für die Individuen der Gesellschaft erreicht werden kann. Als Maßstab für die wohlfahrts-

1) Vgl. dazu insbesondere K. E. Boulding, Welfare Economics, in: A Survey of Contemporary Economics, VoT. II, hrsg. von F. Haley, Homewood, I11. 1952, S. 1 ff.; I. M. D. Little, a.a.0.; E. J. Mishan, A Survey of Welfare Economics 1939-59, in: Economic JournaT, Vol. 70 (1960), S. $197 \mathrm{ff}$.; H. Giersch, Aligeme ine Wirtschaftspolitik. Erster Band: Grundlagen, Wiesbaden 1961, S. 106 ff.; R Jochimsen, Ansatzpunkte der Wohlstandsökonomik, Tübingen 1961. 
maximierende Ressourcenallokation dient der "neueren" Wohlfahrtstheorie ${ }^{1)}$ das nach VILFREDO PARETO benannte Pareto-kriterium. Ist es möglich, durch Änderungen in der Nutzung der Ressourcen - z.B. durch Verschiebungen im Faktoreinsatz, durch Umstellungen in der Produktion oder durch Giutertausch - von einer Situation $A$ zu einem Zustand B überzugehen, der zumindest ein Wirtschaftssubjekt besserstellt ohne gleichzeitig ein anderes Wirtschaftssubjekt schlechter zu stellen, dann wird in der Gesellschaft dieser Zustand B der Situation A vorgezogen. Eine pareto-optimale Ressourcenallokation gilt demnach immer dann als realisiert, wenn es unmöglich geworden ist, irgendein Wirtschaftssubjekt besser zu stellen, ohne gleichzeitig die Position zumindest eines anderen Wirtschaftssubjektes zu verschlechtern.

Die Erreichung des Pareto-0ptimums ist an verschiedene Bedingungen geknüpft, und zwar zunächst an Marginalbedingungen, die gleichzeitig der formal exakten inhaltichen Fassung der verbalen Formulierung des Pareto-Optimums dienen. ${ }^{2)}$ Da die wohlfahrtsökonomischen Marginalbedingungen u.U. lediglich ein lokales Maximum gewährleisten, bedarf es zur Sicherstellung des Pareto-Optimums darüber hinaus der Ergänzung um Totalbedingungen. ${ }^{3)}$ Zu den wichtigsten Totalbedingungen gehören ein gegebener Stand der Technik, eine allgemein akzeptierte Einkommens- und Vermögensverteilung sowie konstante Präferenzen der Individuen.

1) Zu unterscheiden ist zwischen der von Pigou entwickelten "älteren" oder besser utilitaristischen Wohlfahrtstheorie und der auf Pareto aufbauenden "neueren" oder paretianischen Wohlfahrtstheorie. Während Piqou die Mönlichkeit kardinaler Nutzenmessung und interpersoneller Nutzenveraleiche unterstellte und damit die gesellschaftliche Wohlfahrt als Summe der individuellen Wohlfahrten betrachtete, beschränkt sich die paretianische Wohlfahrtstheorie auf ordinale Nutzenmessung und vermeidet Aussagen über eine interpersonelle Nutzenaddition zur Gesamtwohlfahrt. Vql. dazu vor allem I.M.D. Little, a.a.0., S. $11 \mathrm{ff}$.; vgl. auch R. Jochimsen, a.a.0., S. $46 \mathrm{f}$.

2) Ausführlich und anschaulich dazu H. Giersch, a.a.0., S. $106 \mathrm{ff}$., geometrisch E. Sohmen, Allokationstheorie und Wirtschaftspolitik, Tübingen 1976, S. $32 \mathrm{ff}$. und kritisch K. G. Zinn, Allgemeine Wirtschaftspolitik, 2. Auf1., Stuttgart u.a., S. $128 \mathrm{ff}$.

3) Vgl. E. J. Mishan, A Survey of Welfare Economics 1939-59, a.a.0., S. 211; H. Giersch, a.a.0., S. 106 und S. $108 \mathrm{ff}$. 
Soweit das Pareto-Kriterium lediglich als eine Technik zur Beurteilung alternativer wirtschaftlicher Zustände betrachtet wird, gilt das bisher Gesagte unabhängig von der konkreten Ausgestaltung der Wirtschaftsordnung. Das Anliegen der Wohlfahrtsökonomik geht jedoch erheblich weiter. Mit dem Marktpreismechanismus benennt sie gleichzeitig das einzige gesamtwirtschaftliche Koordinationsverfahren, welches stets zum Pareto-0ptimum führt, vorausgesetzt das Marktpreissystem erfiil lt selbst wiederum eine Reihe ganz bestimmter Bedingungen. 1)

Die zentrale Aussage der modernen Wohlfahrtsökonomik lautet nun, daß unter den getroffenen Annahmen - und nur dann - der Marktpreismechanismus die Erfüllung der Marginalbedingungen fur eine pareto-optimale Allokation der Ressourcen gewährleiste und umgekehrt jedes Pareto-optimum zu einem vollkommenen Marktgleichgewicht führe.2)

Ausgehend von diesem wohlfahrtsökonomischen Modell ist es Gegenstand der normativen Theorie der Staatstätiakeit, diejenigen Aufgaben zu identifizieren, die nicht über den Marktpreis-

1) Zu nennen sind zunächst die Voraussetzungen, die das Modell der vollständigen Konkurrenz kennzeichnen, nämlich

- Mengenanpassung seitens aller Marktteilnehmer an die Marktpreise (atomistische Märkte) und

- vollständige Markttransparenz sowie die Erfüllung der Homogenitätsbedingungen (keine sachlichen, persönlichen, räumlichen und zeitlichen Präferenzen).

Darüber hinaus wird unterstellt, daß

- die Produzenten nach Gewinn- und die Konsumenten nach Nutzenmaximierung streben;

- die individuellen Produktions- bzw. Nutzenfunktionen unabhängig sind;

- die Güter und Produktionsfaktoren unendlich teilbar sind;

- die Reaktionsgeschwindigkeit aller Beteiligten unendlich groß ist.

Vgl. hierzu ausführlicher A. E. Ott, Grundzüge der Preistheorie, 2. Auf1., Göttingen 1970, S. 32 f. sowie E. Sohmen, Allokationstheorie und Wirtschaftspolitik, a.a.0., S. 32 und S. $70 \mathrm{f}$.

2) Dieses Dualtheorem wurde erstmals von Arrow und Debreu mathematisch exakt formuliert und bewiesen; vgl. K. J. Arrow und G. Debreu, Existence of an Equilibrium for a Competitive Economy, in: Econometrica, 22. Jg. (1954), S. $265 \mathrm{ff} . ; \mathrm{vgl}$. dazu auch E. Sohmen, Allokationstheorie und Wirtschaftspolitik, a.a.0., S. $68 \mathrm{ff}$. 
mechanismus erledigt, sondern vom Staat wahrgenommen werden sollten.

Sind sämtliche Bedingungen des Konkurrenzmodells erfuillt, so ist ausschließlich die Gewährleistung des geordneten Ablaufs der Marktkoordination - z.B. die Schaffung und Aufrechterhaltung einer Geld- und Rechtsordnung, die Sicherung des Schutzes nach auBen - als öffentliche Aufgabe zu betrachten und damit uiber staatliche Koordinationsmechanismen vorzunehmen.

Ist hingegen eine oder sind mehrere Bedingungen des Konkurrenzmodells verletzt, so ist der Marktpreismechanismus allein nicht mehr in der Lage, die pareto-optimale Allokation der Ressourcen herbeizuführen. Setzen sich z.B. monopolistische Praktiken durch, dann drücken die Preise die Knappheitsrelationen der Güter und Produktionsfaktoren nur unzureichend aus. Ahnliche Konsequenzen haben externe Effekte: Bei der Produktion bzw. Konsumtion bestimmter Guter werden die Produktions-bzw. Konsumtionsbedingungen Dritter berührt, ohne daß dies seinen Niederschlag in den Preisen findet. Die Fälle des "Marktversagens" ${ }^{1}$ ) bieten dann den $A n l a B$ zur Konstituierung weiterer öffentlicher Aufgaben (vgl. Obersicht 14).

Allerdings sind auch innerhalb des wohlfahrtsökonomischen Denkgerüstes verschiedene Ansätze einer öffentlichen Aufgabenerfüllung zur Beseitigung oder Abschwächung der festọestellten Marktmängel zu unterscheiden.

Als konsequentester Weg erweist sich zweifellos die Forderuna, mittels ordnungspolitischer Eingriffe in das marktwirtschaftliche System die Voraussetzungen für die vollständige Konkur-

1) Die paretianische Wohlfahrtsökonomik unterscheidet grundsätzlich zwei Arten des Marktversagens. Zum einen können die Bedingungen des vollkommenen Wettbewerbs verletzt sein. In diesem Fall wird auch von Marktunvoll kommenheiten gesprochen. Zum anderen gibt es zahlreiche Fälle, in denen selbst der vollkommene Konkurrenzmarkt versagt, das Pareto-0ptimum herbeizuführen. Häufig werden lediglich diese Fälle als Marktversagen bezeichnet; so z.B. Bator: "Typically ... we mean the failure of a more or less idealized system of price-market institutions to sustain 'desirable' activities or to estop 'undesirable' activities." F. M. Bator, The Anatomy of Market Failure, QJE Vol. 72 (1958), S. 351. 
Übersicht 14: Wohlfahrtsökonomisches Referenzsystem und öffentliche Aufaaben

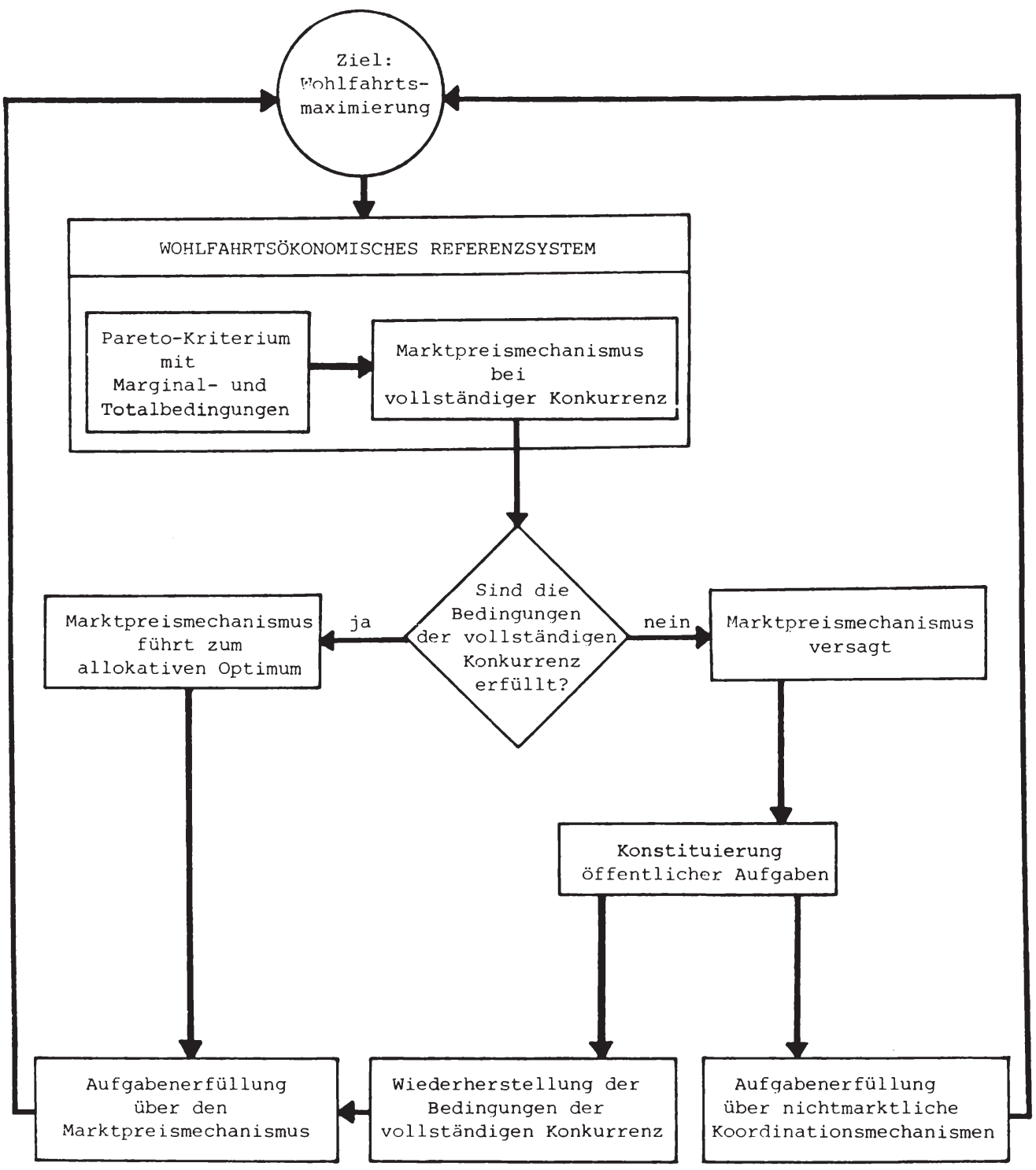


renz durchzusetzen. Dem Staat wird mithin die Aufgabe übertragen, die Marginalbedingungen der optimalen Allokation herzustellen und zu sichern.

Nicht in jedem Fall hält jedoch die Wohlfahrtsökonomik die Heilung der verletzten Marginalbedingungen für die einzig richtige Strategie. Wie die Theorie des "Second Best" nachgewiesen hat, besteht die Möglichkeit, daß mit der Verwirklichung einer Marginalbedingung in einem Teilbereich keine Annäherung an das Pareto-0ptimum einhergeht, wenn in anderen Teilbereichen die Optimalbedingungen nicht realisiert werden können. 1) Das Streben nach einem zweitbesten Optimum schafft allerdings weitere Unvollkommenheiten, so daß bei staatlichen Eingriffen wiederum zusätzliche Nebenbedingungen zu berücksichtigen sind. LIPSEY und LANCASTER haben daraus den Schluß gezogen, daß bei Gültiakeit des Second-Best-Theorems keine verbindlichen wohlfahrtstheoretischen Aussagen mehr möglich sind. 2) Aus dem um SecondBest-überlegungen erweiterten wohlfahrtsökonomischen Ansatz könnten dann auch keine konkreten öffentlichen Aufgaben abgeleitet werden.

In der wohlfahrtstheoretischen Literatur wird deshalb mit der Feststellung der Marktmängel häufig lediglich der lapidare Hinweis verbunden, in den Fällen des Marktversagens müsse die Koordination der Güter und Produktionsfaktoren über den Marktpreismechanismus zugunsten der öffentlichen Bereitstellung abgelöst werden.

1) Unterstellt, ein Teilbereich der Volkswirtschaft ist durch monopolistische Verhaltensweisen geprägt, die durch staatliche Maßnahmen abgebaut werden könnten, während in einem zweiten Teilbereich monopolistische Strukturen vorherrschen, die nicht zu beseitigen sind. Wird nun der Versuch unternommen, im ersten Teilbereich eine Konkurrenzsituation herbeizuführen, so können sich Preisverhältnisse zum zweiten Teilbereich einstellen, die in geringerem Maße als vorher den wahren Knappheitsrelationen entsprechen; vgl. dazu auch B. Külp, Art. Wohlfahrtsökonomik I: Grundlagen, HdWW 9. Bd., Tübingen $1982, S .485$.

2) Vgl. R. G. Lipsey und K. Lancaster, The General Theory of Second Best, REStud 24. Jg. (T956/57), S. 11 ff.; vgl. im Überblick dazu auch U. Schlieper, Art. Wohlfahrtsökonomik II: Theorie des Zweitbesten, HdWW 9. Bd., Tưbingen 1982 , S. $486 \mathrm{ff}$. 
1.2. Zur wirtschaftspolitischen Anwendbarkeit des wohlfahrtsökonomischen Referenzsystems

Es gibt wohl keine zweite ökonomische Teildisziplin, die mit vergleichbarer Leidenschaft entweder attackiert oder verteidigt wird wie die Wohlfahrtsökonomik.

Während die eine Seite die Ansicht vertritt, daß dem wohlfahrtstheoretischen Modell des allgemeinen Konkurrenzgleichgewichts "bisher als Referenzsystem für Allokationsentscheidungen weder an intellektuellen Modellen noch an empirisch getesteten Alternativen etwas annähernd Gleichwertiges an die Seite gestellt werden konnte"1), stößt sie bei der anderen seite auf strikte Ablehnung. Zwar wird ihre formale Eleganz durchaus anerkannt, jedoch ihre praktische Relevanz und politische Durchsetzbarkeit als äuBerst gering veranschlagt, wobei bereits das ParetoKriterium selbst als unzulänglich erachtet oder gleich der gesamte Ansatz als rein formales Blendwerk kritisiert wird, welches sich zudem bei näherer Analyse als methodologisch fragwürdig erweist. 2)

Die extrem divergierenden Auffassungen iuber die Leistungsfähigkeit der paretianischen Wohlfahrtsökonomik sind jedoch zu einem großen Teil auf Mißverständnisse im Hinblick auf die Intention eines Referenzmodells zurückzuführen. Es ist nicht Aufgabe der Wohlfahrtsökonomik, "die Welt zu interpretieren, sondern sie setzt sich das normative Ziel, aufzuzeigen, ob und wie man sie verändern könnte, um sie für die Mitglieder der menschlichen Gesellschaft besser bewohnbar zu machen. Es ist vor allem diese Aufgabe, der unter anderem Gleichgewichtsmodelle vollkommener

1) E. Sohmen, Allokationstheorie und Wirtschaftspolitik, a.a.0., S. 5.

2) So stellt z.B. Jochimsen fest, daß "die Wohlfahrtsökonomik als Wissenschaft bis jetzt nur wenige unmittelbare Ratschläge für die aktuelle und praktische Wirtschaftspolitik gegeben (hat)". R. Jochimsen, a.a.0., S. 5. Noch ablehnender ist die Haltung Häusers: "Sie besticht be $i$ der ersten Begegnung durch ihre Eleganz und eignet sich vorzigilich, um sich damit bewundern zu lassen, aber für den Hausgebrauch ist sie nicht geeignet." K. Häuser, a.a.0., S. 48. Ahnlich auch Boulding: "I believe this attempt has been a failure, though a reasonable glorious one." K. E. Boulding, Economics as a Moral Science, AER Vol. 59 (1969), S. 5. 
Konkurrenz dienen sollen. Sie waren seit jeher als (hypothetische) Referenzsysteme gedacht .... um die Unvollkommenheit der realen welt und die daraus resultierenden Effizienzverluste daran zu messen."1)

Eine auf die unschwer beobachtbare Diskrepanz zwischen Modell und Realität abzielende Kritik geht deshalb weitgehend ins Leere. Berechtigung besitzt jedoch jene Kritik, die auch von Modellen fordert, daß ihre Resultate umsetzbar sind in praktisches Handeln. M.a.W.: Auch Referenzsysteme sollen verwendbar sein zur Gestaltung der sozialen Wirklichkeit. ${ }^{2}$ ) Gerade in dieser Hinsicht muß aber der Anspruch des wohlfahrtstheoretischen Referenzsystems beträchtlich zurückgeschraubt werden.

Im Hinblick auf die Umsetzbarkeit des Referenzsystems in konkrete politische Handlungsanweisungen erweist sich bereits die mangelnde Operationalisierbarkeit der zu maximierenden wohlfahrtstheoretischen Zielgröße als ein zentrales Problem. Das einzelne Wirtschaftssubjekt mag noch in der Lage sein, den Inhalt dessen, was es als ökonomische Wohlfahrt empfindet, hinreichend genau zu umschreiben. Es ist jedoch kein rationales Verfahren denkbar, mit dessen Hilfe offengelegt werden kann, was die Gesellschaft insgesamt materiell unter Wohlfahrt versteht. ${ }^{3)}$ Nichts gewonnen ist auch mit Versuchen, den Begriff "Wohlfahrt" mit ebenso vagen Synonymen wie "Glück" oder "Befriedigung" inhaltlich "auf-

1) E. Sohmen, Allokationstheorie und Wirtschaftspolitik, a.a.0., S. $10 \mathrm{f}$. (Unterstreichungen im Original kursiv).

2) Hier handelt es sich nach Albert um eine Forderung, die allgemein für die Sozialwissenschaft wie für die Nationalökonomie Gültigkeit besitzt; val. H. Albert, Das Ende der Wohlfahrtsökonomik, in: Gewerkschaftliche Monatshefte, 1958, S. 33. In diesem Sinne ist wohl auch die zitierte Kritik von Jochimsen und Häuser zu verstehen.

3) Auf diesen Sachverhalt hat insbesondere Arrow in aller Deutlichkeit hingewiesen; vgl. K. J. Arrow, Social Choice and Individual Values, a.a.0., S. $22 \mathrm{ff}$.; vgl. hierzu auch den zweiten Teil, Abschnitt I, Punkt 3.2.2. dieser Arbeit. 
zufiullen". 1) Es ist deshalb schwierig, den Aussagegehalt der Wohlfahrtsökonomik zu überprüfen, denn während im Rahmen der positiven okonomik die Ergebnisse der Theorie getestet werden können, ist es in der Wohlfahrtstheorie lediglich möglich, die dort gesetzten Annahmen kritisch zu hinterfragen.

Entgegen der Ansicht vieler Wohlfahrtsökonomen ist das ParetoKriterium keineswegs frei von Werturteilen. ${ }^{2)}$ Es wird freilich unterstellt, daß die Forderung, ein gesellschaftlicher Zustand sei einem anderen Zustand immer dann vorzuziehen, wenn zumindest ein Wirtschaftssubjekt besser gestellt werde, ohne gleichzeitig ein anderes schlechter zu stellen, in der Gesellschaft auf allgemeine Akzeptanz stößt. ${ }^{3)}$ Damit tritt der neuralgische Punkt des wohlfahrtsökonomischen Modells offen zutage, denn durch die Annahme, mit jeder Allokationsentscheidung stelle sich uno actu eine allgemein akzeptierte Einkommensverteilung ein, wird die gesamte Verteilungsproblematik - zumindest im

1) Unter diesen Versuchen ist wohl das Benthamsche Prinzip, das gesellschaftliche Wohlfahrtsmaximum als "das größte Glück der größten Zahl" zu interpretieren, der bekannteste. Diese normative Glücksregel bildete auch den gedanklichen Ausgangspunkt für die auf Grundlage kardinaler Nutzenmessung und interpersoneller Nutzenvergleiche entwickelten pigovianischen (utilitaristischen) Wohlfahrtstheorie. Kritisch dazu vor allem I. M. D. Little, a.a.0., S. $7 \mathrm{ff}$.

2) Vgl. K. E. Boulding, Economics as a Moral Science, a.a.0., S. 1 ff.; E. Lauschmann, Grundlagen, Grenzen und Entwicklungsmöglichkeiten der WeTfare Economics, in: Probleme der normativen okonomik und der wirtschaftlichen Beratung, hrsg. von E. von Beckerath und H. Giersch, SchdVSocpol N.F., Bd. 29, Berl in 1963, S. 109.

3) Diese Ansicht vertreten Z.B. auch E. J. Mishan, A Survey of Welfare Economics 1939-59, a.a.0., S. 199 sowie K. J. Arrow, Social Choice and Individual Values, a.a.0., S. 96. 
Standardmodell der Wohlfahrtsökonomik - ausgeblendet. 1) Nicht selten wird argumentiert, eine solche Annahme sei unumgänglich, da die Suche nach der "gerechten" Verteilung eine reine Wertungsfrage sei, die die Kompetenz der Wissenschaft überschreite. Dieser Vorstellung ist jedoch entgegenzuhalten, daß auch andere wirtschaftspolitische Ziele, wie $z$.B. eine optimale Allokation oder ein stabiles Preisniveau letztlich nicht ohne die Setzung eines Werturteils zu formulieren sind.

Das Pareto-Kriterium erfordert stets einstimmige Entscheidungen (bei Nichtberücksichtigung von Enthaltungen), d.h. jedes Individuum kann ständig von seinem Vetorecht Gebrauch machen. Daher läßt das Pareto-Kriterium nur äußerst schmalen Raum für politische Veränderungen, statt dessen trägt es vielmehr regelrecht zu einer Zementierung des status quo bei. Für JOCHIMSEN ist deshalb das Pareto-Kriterium auch "ein beinahe jede prakti-

1) Vereinzelt hat es allerdings Ansätze gegeben, die Verteilungsaspekte auch explizit im wohlfahrtstheoretischen Modell zu berücksichtigen, indem das Pareto-Kriterium selbst auf eine breitere Basis gestellt wird. Einer dieser Ansätze besteht in der Einführung von Kompensationszahlungen. Nach dem Kaldor-Hicks-Kriterium werden wirtschaftspolitische Maßnahmen auch dann als pareto-optimal bezeichnet, wenn durch sie einige Individuen besser und andere schlechter gestellt werden, die Begünstigten aber ihre Nutzengewinne dazu verwenden können, die Benachteiligten für ihren Nutzenentgang zu entschädigen. Die Kritik Scitovskys, die Anwendung des von Kaldor und Hicks entwickelten Kompensationskriteriums könne zu logischen Widersprüchen führen, hatte die Entwicklung weiterer Kompensationskriterien zur Folge (Gorman, Samuelson), die zwar unter ganz bestimmten Voraussetzungen widerspruchsfreie Ergebnisse lieferten, jedoch nur um den Preis einer erheblichen Einschränkung ihres Anwendungsbereiches. Dies stand aber ganz im Gegensatz zur ursprünglichen Intention einer Ausweitung der wirtschaftspolitischen Umsetzungsmöglichkeiten des Pareto-Kriteriums. Damit wurden die eigentlich strittigen Probleme weiterhin ausgeklammert. Ausführlich zur Darstellung und kritischen Beurteilung der verschiedenen Kompensationskriterien vgl. E. J. Mishan, A Survey of Welfare Economics 1939-59, a.a.0., S. $218 \mathrm{ff}$.; E. Sohmen, Grundlagen, Grenzen und Entwicklungsmögl ichkeiten der Welfare Economics, a.a.0., S. $75 \mathrm{ff}$.; B. Külp, Die Rolle der Einkommensverteilung innerhalb der Wohlfahrtstheorie, a.a.0., S. $108 \mathrm{ff}$.

Neuere Ansätze einer pareto-konformen Berücksichtigung der Verteilunasproblematik gehen davon aus, daß selbst bei Aufrechterhaltung der Annahme egoistischer Verhaltensweisen der Individuen Bezieher höherer Einkommen häufig freiwillig zu einer Umverteilung zu ihren Lasten bereit sind, weil sich dadurch auch ihr eigener Nutzen erhöht, es mithin ökonomisch rational ist, einer Belastung zuzustimmen. Grundlegend dazu H. M. Hochman und J.D. Rodgers, Pareto Optimal Redistribution, AER Vol. 59 (1969), S. $542 \mathrm{ff}$. 
sche Wirtschaftspolitik verhindernder Maßstab für wirtschaftliche Reorganisationen."1)

Da in der Wohlfahrtsökonomik die ihr zugrundeliegenden Annahmen besondere Bedeutung erlangen, ist auffällig, daß zwar der Darstellung und Würdigung der Marginalbedingungen im allgemeinen breiter Raum gewidmet wird, die Totalbedingungen hingegen trotz ihrer vergleichsweise wesentlich bedeutsameren Rolle für die praktische Wirtschaftspolitik ${ }^{2}$ i.d.R. nur der Vollständigkeit halber Erwähnung finden. ${ }^{3)}$ Diese Vorgehensweise mag zwar einerseits auf ein gewisses Verständnis stoßen, sind doch die Totalbedingungen z.T. nur unter großen Schwierigkeiten mit Hilfe rein ökonomischer Kategorien faßbar. Es bleibt aber dennoch überraschend angesichts der drastischen Einschränkungen, die die Aussagekraft des Pareto-Kriteriums durch sie zwangsläufig erfährt. Mit Setzung der Marginalbedingungen werden zwar die Voraussetzungen zur Erreichung eines stationären, d.h. durch schrittweise Reallokation des - insbesondere durch eine gegebene Ausstattung an Produktivkräften und einen gegebenen Stand der Technik - vorhandenen Bestandes an Ressourcen erreichbaren Optimums umschrieben, offen bleibt aber die Frage, auf welchem Wege das gesamtwirtschaftliche Optimum realisierbar ist, sobald infolge technischen Fortschritts z. B. neue Produkte entwickelt oder Produktionsverfahren verbessert werden, rechtliche Neuregelungen veränderte Rahmenbedingungen schaffen und sich dadurch Verschiebungen in der Einkommens-

1) R. Jochimsen, a.a.0., S. 56.

2) So bezweifelt z.B. Boulding, daß die Kenntnis der sieben Marginalbedingungen sich für den Politiker überhaupt jemals als wertvoll erwiesen hat; vgl. K. E. Boulding, Welfare Economics, a.a.0., S. 32.

3) Disproportionalitäten in der wissenschaftlichen Behandlung der Marginalund Totalbedingungen betont auch H. Giersch, a.a.0., S. 109; Sohmen ist sogar der Ansicht, daß "wir in der Frage der Totalbedingungen fast völlia im Dunkeln (tappen)". E. Sohmen, Grundlagen, Grenzen und Entwicklungsmöglichkeiten der Welfare Economics, a.a.0., S. 78. 
und Vermögensverteilung sowie Variationen in den Präferenzen der Wirtschaftssubjekte ergeben.

Als Zwischenresümee drängen sich bereits wenig positive Schlußfolgerungen auf: Ist das wohlfahrtsökonomische Referenzsystem bereits aufgrund der realitätsfernen Annahme ganz allgemein für wirtschaftspolitische Zwecke kaum verwendbar, so ist es offenbar völlig überfordert, im Hinblick auf die durch technischen Fortschritt, neue rechtliche Bestimmungen, modifizierte individuelle Präferenzen etc. entstehenden neuen bzw. veränderten Aufgaben Kriterien für eine ökonomisch zweckmäßige Verteilung dieser Aufgaben zwischen privatem und öffentlichem Sektor zu liefern.

Schließlich leidet das wohlfahrtsökonomische Modell unter einem weiteren Mangel. Es nimmt ausschließlich einen Vergleich der realen wirtschaftichen Situationen mit den theoretischen Idealbedingungen vor. Nachdem - ausgehend vom allgemeinen Konkurrenzgleichgewicht - die Fälle abgeleitet sind, in denen der Marktpreismechanismus "versagt", eine effiziente Allokation herbeizuführen, wird ohne weitere Prüfung der allgemeine Schluß gezogen, durch statliche Eingriffe könne die gewünschte A1lokationseffizienz gewährleistet werden. Dieser Schluß ist äußerst fragwürdig, wird doch fälschlicherweise der Eindruck erzeugt, die relevante Entscheidung sei diejenige zwischen einem mit Mängeln behafteten und einem ideal funktionierenden Koordinationsmechanismus. DEMSETZ hat eine solche Vorgehensweise treffend als "Nirwana-Ansatz" bezeichnet: "In practise, those who adapt the nirvana viewpoint seek to discover discrepancies between the ideal and the real and if discrepancies are found, they deduce that the real is inefficient."1) ob die Marktlösungen durch staatliche Eingriffe zu verbessern

1) H. Demsetz, Information and Efficiency: Another Viewpoint, JlawEc 11. Ja. (1969), S. 1. Ahnlich bereits R. Coase, The Problem of Social Cost, JLawEC 3. Jg. (1960), S. 18. 
sind, ist deshalb nicht allein durch eine Analyse der Funktionsmängel des Marktes feststellbar, sondern bevor solche Schlüsse gezogen werden können, müssen die Resultate des statijchen Handelns einer ebenso eingehenden Prüfung unterzogen und mit der Marktlösung verglichen werden. Besteht mithin die einzig akzeptable Vorgehensweise darin, realisierbare Alternativen zu vergleichen ${ }^{1)}$, so ist die Untersuchung entsprechend auszuwe $i$ ten:

- Den Kosten, die der Volkswirtschaft infolge der fehlerhaften Funktionsweise des Marktpreissystems erwachsen, sind die gesamtwirtschaftiichen Kosten einer Korrektur der Marktschwächen gegenüberzustellen. Dabei ist zu berücksichtigen, daß es zur übertragung einer Aufgabe auf den Staat grundsätzlich eine Reihe abgestufter Alternativen gibt, die in sehr unterschiedlichem Maße Kosten verursachen, und darüber hinaus ist zu bedenken, daß jeder staatliche Eingriff ebenfalls lediglich suboptimale Ergebnisse produziert, mit der Folge, daß weiterhin gesamtwirtschaftliche Kosten anfallen.

- Umgekehrt gilt, daß nicht nur die Funktionsdefizite des Staates zu Kostenbelastungen für die Volkswirtschaft führen, sondern ebenso auch sämtliche, auf eine Beseitigung der Defizite abzielenden Reorganisationen, wobei keine reale Marktlösung denkbar ist, die frei von gesamtwirtschaftlichen Kosten wäre. 2)

Ganz offensichtlich ist bei einer derart ausgeweiteten Analyse das wohlfahrtsökonomische Referenzsystem der Fähigkeit beraubt, als Entscheidungshilfe für eine zweckmäßige Aufgabenverteilung zwischen privatem und öffentlichem Sektor zu fungieren. Mehr

1) Demsetz bezeichnet diesen Ansatz als "comparative institution approach"; vgl. H. Demsetz, a.a.0., S. 1.

2) Gerade in der Privatisierungsdebatte scheint der Nirwana-Ansatz weit verbreitet zu sein, ist doch die Aufdeckung staatlicher Ineffizienzen hier häufig gleichbedeutend mit der Proklamation eines übergangs zum - offenbar als fehlerfrei funktionierend angesehenen - Marktpreissystem. 
noch: Es verhindert geradezu eine rationale Entscheidungsfindung, da es auf der ständigen Suche nach optimalbedingungen den Blick für das Machbare, d.h. für brauchbare Entscheidungen verliert.

\section{Gesellschaftliche Ziele als Referenzsystem}

Der verlockende Versuch, mit Hilfe des wohlfahrtsökonomischen Modells Bestlösungen abzuleiten, somit Abweichungen von einem optimum feststellen zu können und auf diesem Wege dem öffentlichen Sektor klar umrissene Aufgaben zuzuweisen, schlägt also fehl. Als Entscheidungshilfe für eine Aufgabenzuweisung zum privaten bzw. öffentlichen Sektor der Volkswirtschaft erweist sich das Modell vor allem deshalb als ungeeignet, weil

- das Pareto-Kriterium Effizienzbedingungen für ein inhaltsleeres Zielsystem formuliert und daruber hinaus nur in einem äußerst engen Rahmen wirtschaftliche Reorganisationen zuläßt,

- die Realisierung der wohlfahrtsökonomischen Marginalbedingungen lediglich ein lokales Optimum gewährleistet und damit zu einer rein statischen Betrachtungsweise führt und

- von der Tatsache abstrahiert wird, daß sämtliche wirtschaftlichen Reorganisationen Kosten verursachen und zudem stets nur neue Suboptimalitäten hervorbringen können.

GERHARD COLM hat mit der National Goals Analysis ein Konzept entwickelt, das er selbst als einen alternativen Ansatz zum wohlfahrtsökonomischen Modell bezeichnet und für theoretisch weniger ambitiös, dafür aber mit dem Vorteil der praktischen Anwendbarkeit ausgestattet hält. 1) Wir sehen in der Vorgehensweise COLMS jedoch weniger eine Alternative als vielmehr eine notwendige Ergänzung zur Wohlfahrtsökonomik.

1) Vgl. G. Colm, National Goals Analys is and Marginal Utility Economics, a.a.0., S. $216 \mathrm{ff}$. 
Bei COLM bilden die von der Gesellschaft verfolgten Ziele das Referenzsystem zur Ableitung von Kriterien für die Verteilung der Aufgaben in der Volkswirtschaft, d.h. die Aktivitäten privater und öffentlicher Aufgabenträger, die Qualitäten marktlicher und nichtmarktlicher Koordinationsmechanismen sowie der Einsatz staatlicher Instrumente sind unter dem Gesichtspunkt zu untersuchen, welchen Beitrag sie zur Verwirklichung der gesellschaftlichen Ziele zu leisten vermögen (siehe übersicht 15). Die gesellschaftlichen ziele sind dabei weder gleichzusetzen mit der Summe der persönlichen Präferenzen aller Wirtschaftssubjekte noch werden sie allein von einer bewußt in die konsumentensouveränität der Individuen eingreifenden, mit herausragenden Einsichten ausgestatteten Elite formuliert, sondern sie sind, wie COLM darlegt, das Resultat eines "kreativen Prozesses", der sich aus dem Zusammenspiel der verschiedenen Akteure des Marktes und der politischen Entscheidungsträger ergibt. 1 )

Die Konzeption COLMS scheint somit - zumindest auf den ersten Blick - einige der Lücken zu schließen, die die Wohlfahrtsökonomik hinterläßt:

- Während das Zielsystem der Wohlfahrtsökonomik inhaltlich unbestimmt bleibt, fällt im Rahmen der National Goals Analysis der wissenschaftlichen Beratung der Politik die Aufgabe zu, die vagen gesellschaftlichen Zielvorstellungen $z u$ "interpretieren", d.h. mit quantifizierbaren Daten zu füllen, und realisierbare Alternativen aufzuzeigen. Diese Art der Zielerforschung (goals research) ist für COLM von "operationalem" Wert, da "it permits a study of quantifiable magnitudes and their relationship to each other." 2 )

- Mit dem Hinweis auf den zur Zielfindung notwendigen "kreativen Prozeß", an dem alle Akteure der Gesellschaft zu beteiligen sind, werden die im wohlfahrtsökonomischen Modell als

1) Vgl. G. Colm, National Goals Analysis and Marginal Utility Economics, a.a.0., S. 217.

2) Ebenda, S. 220. 
Ubersicht 15: Gesellschaftliche Ziele als Referenzsystem und öffentliche Aufgaben

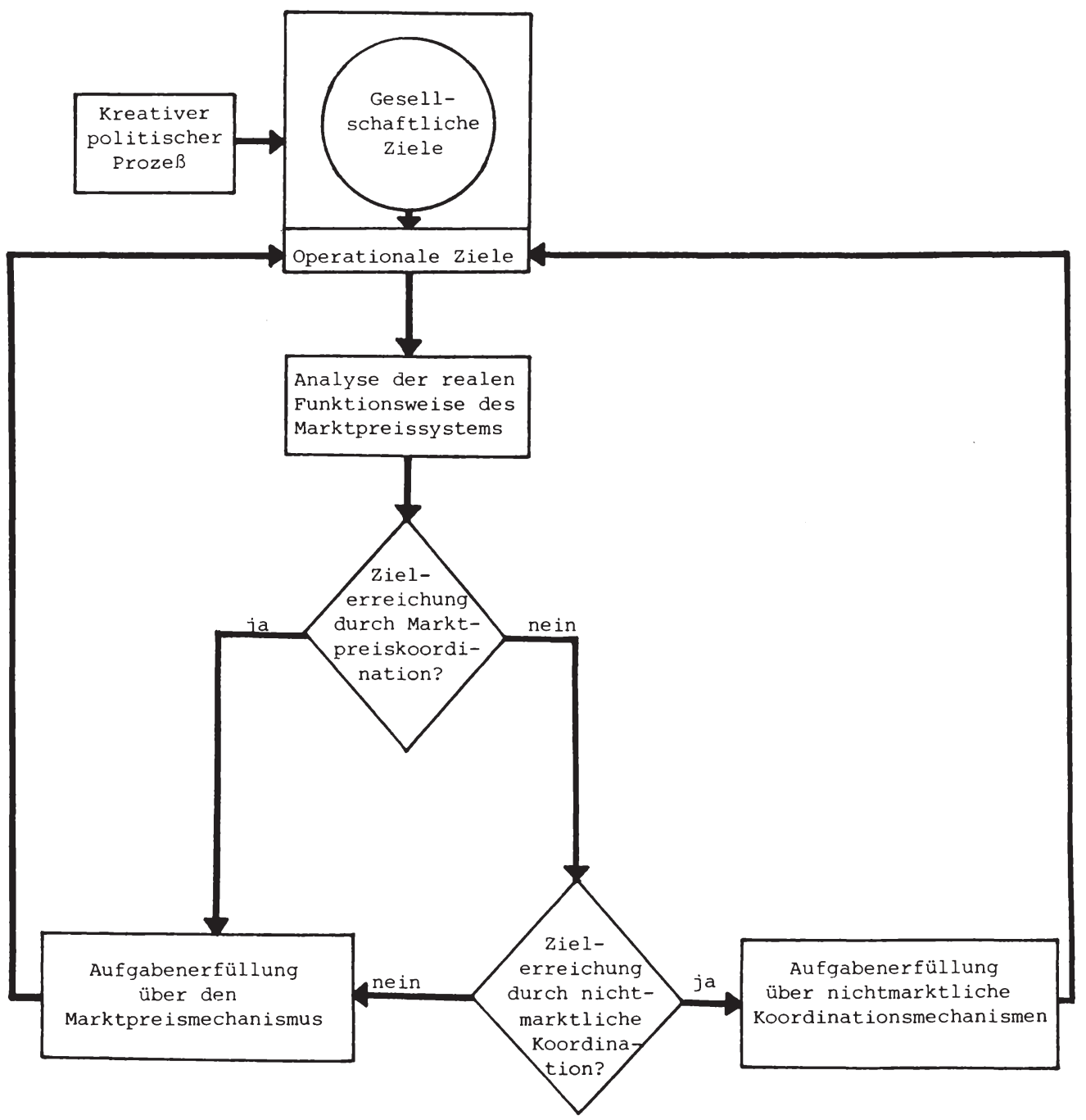


gegeben angenommenen Totalbedingungen zu jederzeit gestaltbaren Variablen im Hinblick auf ihren Beitrag zur Verwirklichung gesellschaftlicher Ziele. Damit erhält die Analyse dynamischen Charakter. ${ }^{1)}$

- COLM fordert, daß zunächst die Möglichkeiten und Mängel des Marktpreissystems und darauf aufbauend die erwünschten und unerwünschten Erscheinungen der statlichen Güterversorgung zu untersuchen sind. Diese Untersuchung sei jedoch nur in Verbindung mit den gesellschaftlichen Zielen sinnvol1.2) Der Analyse der Marktmängel ist also zunächst eine Beurteilung der Leistungsfähigkeit alternativer politischer Lösungen in bezug auf die Verwirklichung der angestrebten Ziele gegenüberzustellen, bevor eine endgültige Entscheidung über Art und Weise der Aufgabenerfüllung gefällt werden kann. Mit dieser Vorgehensweise wird der "Nirwana"-Ansatz der Wohlfahrtsökonomik vermieden.

Der von COLM entworfene Ansatz bietet zwar die Richtpunkte für ein sachgerechtes Vorgehen, seine Schwäche lieat aber darin, daß die Aussagen an den entscheidenden Stellen äußerst vage bleiben:

- Methodische Probleme sind bereits im Zielfindungsprozeß selbst angelegt, da Zielstrukturen stets horizontale und vertikale Interdependenzen aufweisen. ${ }^{3)}$ Mit diesen Problemen setzt sich COLM freilich nicht auseinander, sondern stellt lediglich fest: "I believe that the concepts of the general interest and of goals can be more easily filled with quantifiable data than the concept of marginal individual preference schedules." 4)

1) Vgl. S. Katterle, Wohlfahrtsökonomik und Theorie der Staatswirtschaft, in: Finanzarchiv, N.F., Bd. $30(1971 / 72)$, S. $20 \mathrm{f}$.

2) Vgl. G. Colm, National Goals Analysis and Marginal Utility Economics, a.a.0., S. 222.

3) Auf den Aufbau sowie die Möglichkeiten und Grenzen solcher Zielstrukturen wurde bereits im ersten Tei1, Abschnitt I. 2. ausführlich eingegangen.

4) G. Colm, National Goals Analysis and Marginal Utility Economics, a.a.0., S. 219 . 
- COLM verweist im Zusammenhang mit der Zielfindung auf den "kreativen Prozeß" der an der politischen Entscheidungsbildung beteiligten Akteure. Damit trägt er zwar "der aktiven Funktion von politischen Instanzen und offentlichkeit im Prozeß der demokratischen Willensbildung Rechnung" ${ }^{1)}$, ohne weitere Erklärungen der Funktionsweise dieses kreativen Prozesses verbleiben aber die eigentlichen Probleme in der "black box" der Politik.

- Indem COLM verlangt, die Mängel der Marktpreiskoordination den Funktionsdefiziten der statlichen Allokation gegenüberzustellen, wird der "Nirwana"-Ansatz vermieden. Offen bleibt jedoch, anhand welcher Kriterien festgestellt werden kann, was als Mangel oder als Funktionsdefizit im jeweiligen system anzusehen ist. COLM weist lediglich lapidar darauf hin, daß die Kriterien sich aus der Beziehung zwischen den Leistungen beider systeme und den gesellschaftlichen Zielen ergeben. ${ }^{2}$ )

\section{Leitlinien eines Referenzsystems für realisierbare}

\section{Alternativen}

Die Ausführungen haben gezeigt, daß sich weder das Modell der Wohlfahrtsökonomik noch die Konzeption der gesellschaftlichen Ziele als alleinige Entscheidungsgrundlage für eine Aufgabenverteilung zwischen dem privaten und dem öffentlichen Sektor eignet. Die Kritik an beiden Ansätzen könnte jedoch die Wegmarkierungen für ein Referenzsystem liefern, das von vornherein auf realisierbare Alternativen ausgerichtet ist. Die Leitlinien zur Beantwortung der Frage nach der ökonomisch zweckmäßigen Aufgabenverteilung in der Marktwirtschaft sind dann in drei Schritten zu erarbeiten (siehe übersicht 16):

1) S. Katterle, a.a.0., S. 21.

2) Vgl. G. Colm, National Goals Analys is and Marginal Utility Economics, a.a.0., S. 217 . 
Übersicht 16: Ein Referenzsystem für realisierbare

Alternativen und öffentliche Aufaaben

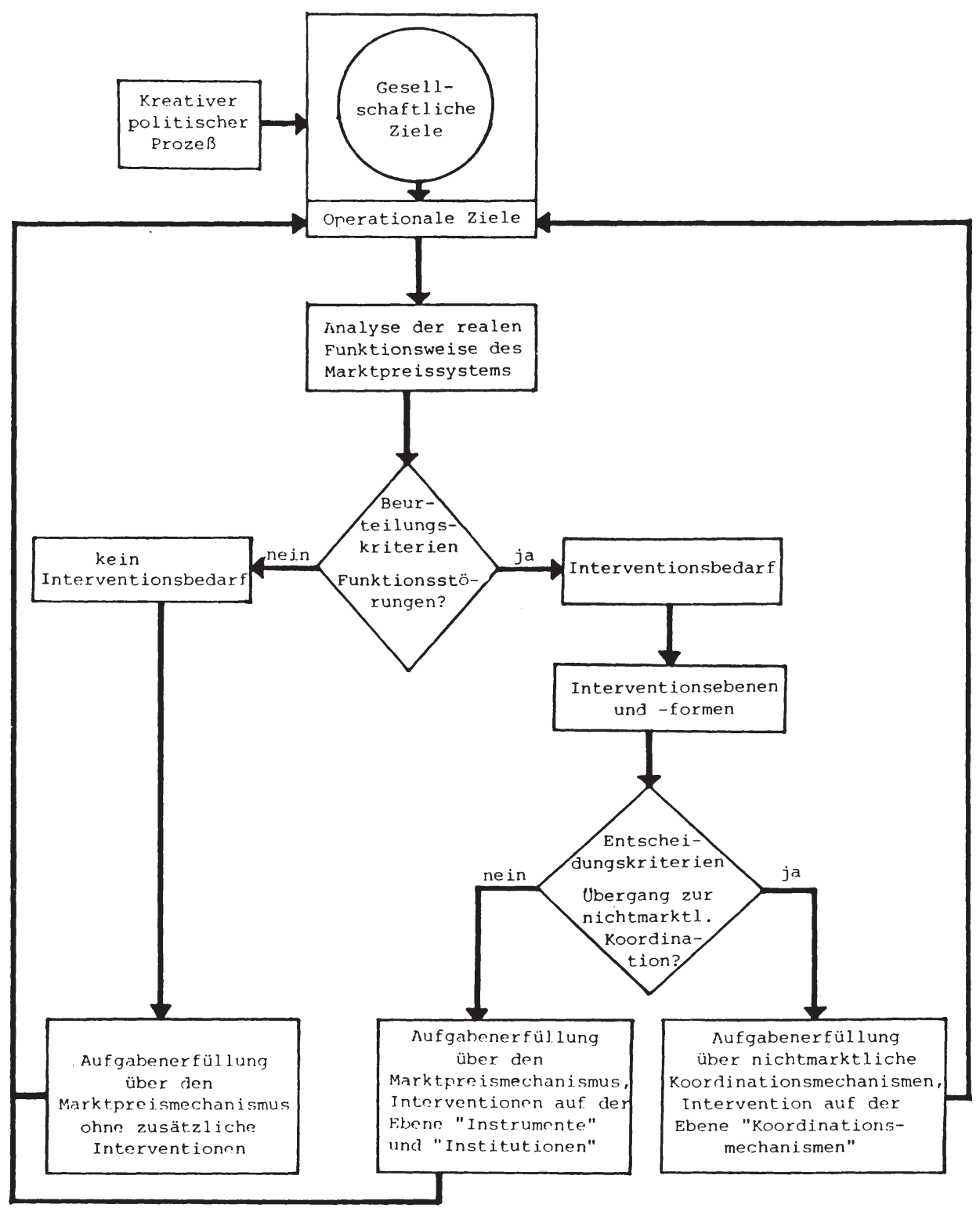


- In einem ersten Schritt müssen ökonomische Kriterien entwickelt werden, mit deren Hilfe Funktionsmängel des Marktpreissystems festgestellt werden können. Dabei handelt es sich um Beurteilungskriterien, die als Legitimationsgrundlage für einen öffentlichen Eingriff in den Markt zu betrachten sind.

- In einem zweiten Schritt ist eine Analyse der prinzipiell denkbaren Interventionsformen vorzunehmen. Sie darf sich jedoch nicht auf die verschiedenen staatlichen Instrumente beschränken, sondern muß auch die Bandbreite öffentlicher und privater Institutionen umfassen sowie die unterscheidbaren nichtmarktlichen Koordinationsmechanismen einbeziehen.

- In einem dritten Schritt hat schließlich die Auswahl der geeigneten Interventionsformen anhand von Entscheidungskriterien zu erfolgen. Als Grundlage dienen hier verschiedene gesamtwirtschaftliche Kostenkategorien.

\section{1. Beurteilungskriterien}

Den analytischen Ausgangspunkt bildet die reale - also gegenwärtig in der Bundesrepublik Deutschland beobachtbare - Ar.t und Weise der marktwirtschaftlichen Koordination von Gütern und Dienstleistungen. Weist das Marktpreissystem Funktionsstörungen auf, ist zu prüfen, in welchen Fällen durch öffentliche Interventionen Verbesserungen der Ausgangsposition zu erzielen sind. Diese Vorgehensweise empfiehlt sich sowohl aus ordnungspolitischen als auch aus pragmatischen Erwägungen. In einem marktwirtschaftlichen system duirfte es geradezu selbstverständlich sein, daß der öffentliche Sektor mit der Beweislast beschwert wird, "bessere" Koordinationsleistungen vollbringen zu können als der Marktpreismechanismus. ') Das markt-

1) "Dies ist ... ein für eine historische Marktwirtschaft sozusagen natürlicher Ausgangspunkt, wie es umgekehrt in einer zentral geleiteten Staatswirtschaft offenbar erst des Nachweises von Mängeln bedarf, bevor marktwirtschaftliche Elemente eingeführt werden." G. Krause-Junk, Probleme der Berechnung und Schätzung öffentlicher Ausqaben, in: Public Finance, 27. Jg. (1972), S. 130. 
wirtschaftliche System wird überdies deshalb als Ausgangspunkt der Analyse gewählt, weil die Grundfunktionen statichen Handelns häufig nur unzureichend aus sich selbst heraus erklärbar sind. Sie werden oftmals erst aus ihren Rahmen- und Eraänzungsfunktionen zum Marktpreismechanismus verständlich. In vielen Fällen hat ein - U.U. lediglich vermutetes - Versagen des Marktes den Stat erst auf den Plan gerufen. Das Anwachsen statlicher Aktivitäten ist demnach das Resultat tatsächlicher oder vermuteter Grenzen marktwirtschaftlicher Problemlösungen. Folglich sprechen gute Gründe dafür, das Marktpreissystem zum Ausgangspunkt der Untersuchung zu macher und die Araumente, die in der Vergangenheit für eine Öbernahme bestimmter Aufgaben durch den öffentlichen Sektor ins Feld geführt wurden, kritisch zu analysieren.

Ist der analytische Ausgangspunkt festgelegt, so sind zunächst die Bereiche wirtschaftlicher Tätigkeit, in denen ein Marktversagen vermutet wird, zu identifizieren. Genauer: Es sind die ökonomischen kriterien zu benennen, die Funktionsstörungen des Marktpreissystems belegen. Diesen Kriterien darf allerdings nicht von vornherein die Rolle von Entscheidungskriterien für eine Aufgabenverteilung zugewiesen werden. Sie können vielmehr nur die Legitimationsgrundlage für öffentliche Interventionen bilden, d.h. sie sind als Prüf-oder Beurteilungskriterien und damit als notwendige Bedingungen für einen Eingriff in den Marktpreismechanismus und gegebenenfalls für eine Ablösung des Marktpreissystems zu betrachten. Ob und in welcher Form eine Intervention zu erfolgen hat, ist erst anhand weiterer, hinreichender Kriterien zu entscheiden.

Einige der in der wohlfahrtsökonomischen Literatur diskutierten Fälle des Marktversagens können dabei als Beurteilungskriterien erneut aufgenommen werden. Mängelanalysen wurden für den allokativen Bereich vor dem Hintergrund verschiedener fundamentaler theoretischer Ansätze wie u.a. der Theorie der öffentlichen Güter (Samuelson, Musgrave), der Anatomie des 
Marktversagens (Bator) oder dem Konzept der externen Effekte (Mishan, Head) sowie deren Weiterentwicklungen erstellt. Diese Ansätze beschränken sich jedoch immer auf die Ableitung ausgewählter Kategorien von Marktmängeln. Deshalb ergibt sich nicht nur die Notwendigkeit, zusätzliche Beurteilungskriterien in das bestehende Denksystem einzufügen, sondern es ist auch unerläßlich, den Argumentationsrahmen in verschiedene Richtungen zu erweitern. Wie gezeigt, gilt dies in erster Linie für die von der Wohlfahrtsökonomik gesetzten Totalbedingungen.

Die Kriterien zur Beurteilung von Marktmängeln sollen systematisch dargestel1t werden. Das verlangt zum einen die Erfassung möglichst vieler Fälle, in denen Funktionsstörungen des Marktpreissystems konstatiert werden können, zum anderen die theoretische Einordnung dieser Fälle sowie die Offenlegung von Gemeinsamkeiten und die Herausstellung von prinzipiellen Unterschieden, mithin also eine Typologisierung der Beurteilungskriterien.

Die Systematisierung der Beurteilungskriterien wird nach folgenden Gesichtspunkt vorgenommen.

Nach dem Ort der Entstehung eines Marktmangels wird differenziert zwischen

1. Marktversagen auf der Angebotsseite des Wirtschaftsprozesses und

2. Marktversagen auf der Nachfrageseite des Wirtschaftsprozesses.

Mit der Unterscheidung zwischen auf der Angebotsseite verursachten Marktmängeln einerseits und auf der Nachfrageseite entstandenen Funktionsstörungen des Marktes andererseits wird zu einer primär prozessualen Sichtweise übergegangen und damit der enge Ansatz, die Funktionsfähigkeit des Marktpreissystems allein durch technische Eigenschaften der Güter und Dienstleistungen zu determinieren, überwunden. 


\subsection{Interventionsebenen und -formen}

Sind anhand der Beurteilungskriterien ein Marktversagen oder allgemeiner Funktionsstörungen des Marktpreissystems konstatierbar, so wird damit ein Interventionsbedarf begrüdet. Unter Interventionsbedarf ist dabei ausschließlich die Notwendigkeit eines öffentlichen Eingreifens zu verstehen, ohne daß gleichzeitig die materielle Ausgestaltung des öffentlichen Tätigwerdens in irgendeiner Weise präjudiziert wird. ${ }^{1}$ Mit der Feststellung eines Interventionsbedarfes bleibt demnach die Beantwortung folgender Fragen noch völlig offen:

- In welchen Fällen können Funktionsmängel des Marktes durch den Einsatz staatlicher Instrumente korrigiert werden? Zur Verfügung steht dabei eine Vielzahl regulativer, finanzieller und informatorischer Instrumente des staates. 2) Inwieweit kann also mit Hilfe des traditionellen wirtschaftspolitischen Instrumentariums in den Markt eingegriffen, die Gïterbereitstellung aber weiterhin über den Marktpreis gesteuert werden?

- Soll die Güterbereitstellung weiterhin privaten Institutionen uberlassen bleiben oder aber öffentlichen Institutionen uberantwortet werden? Auch hier gilt es, eine Vielzahl privater Aufgabenträger (neben Unternehmen u.a. auch freie Berufe, Verbände, Selbstorganisationen) und öffentliche Träger zu unterscheiden. 3 )

1) Im Vergleich zum Regelungsbegriff hält auch Mayntz den Begriff der Intervention für den umfassenderen, da er nicht auf Ziel und Art des Tätigwerdens, sondern auf das Tätigwerden als solches abstellt; vgl. R. Mayntz, Sachverständigenanhörung zu Ursachen einer Bürokratisierung in der öffentlichen Verwaltung sowie zu ausgewählten Vorhaben zur Verbesserung des Verhältnisses von Bürger und Verwaltung am 19. und 20. Juni 1980 in Bonn, Teil C: Wissenschaftliche Auswertung Gesetzgebung und Bürokratisierung, Bonn 1980, S. 25.

2) $\mathrm{Vgl}$. dazu den überblick über die staatlichen Instrumente im zweiten Teil, Abschnitt II.4.

3) Zur Typologie öffentlicher und privater Aufgabenträaer vql. zweiten Teil, Abschnitt II.2. 
Übersicht 17: Interventionsebenen und -formen

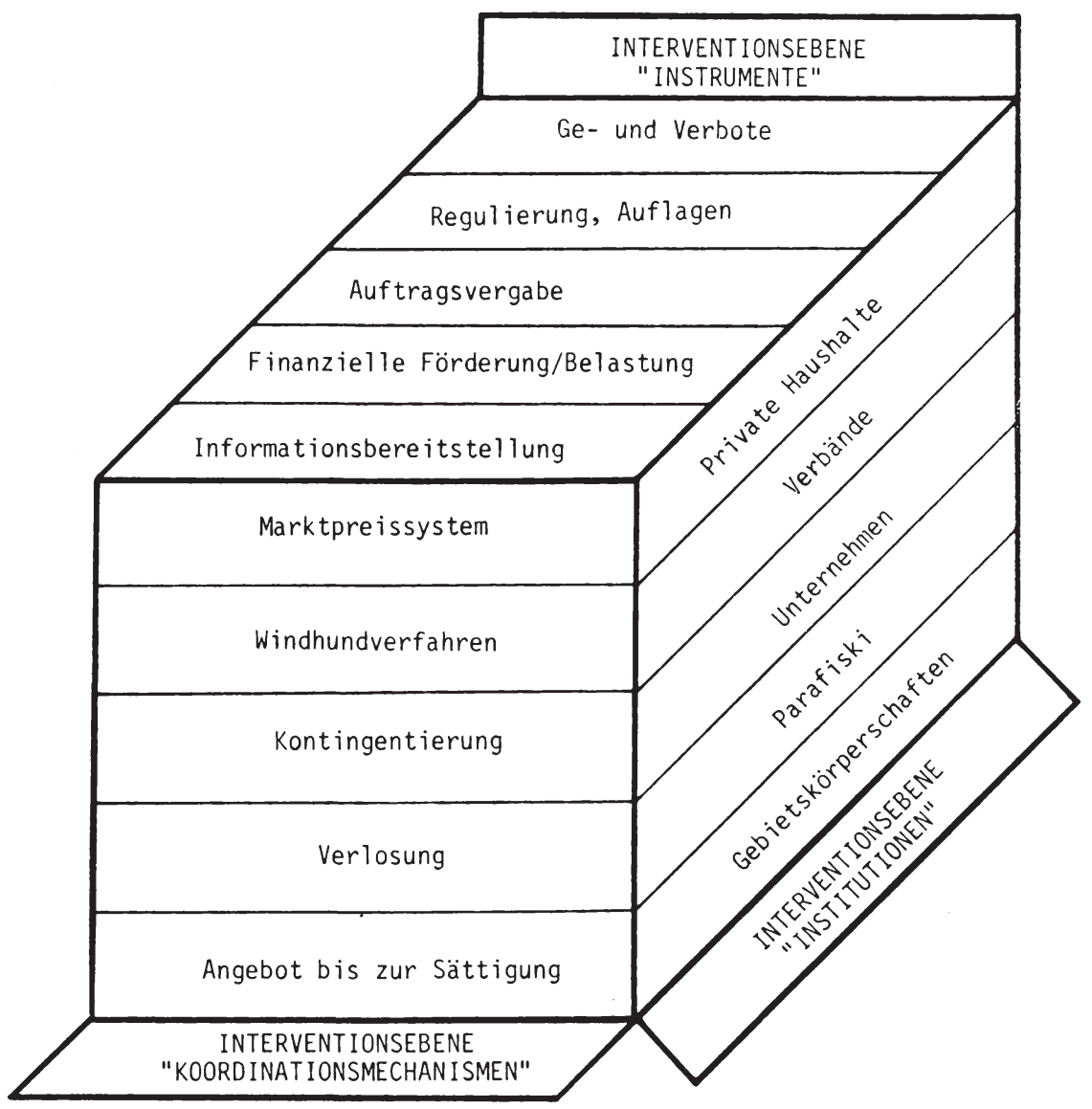


- In welchen Fällen ist der übergang zu öffentlichen - oder besser: nichtmarktlichen - Koordinationsmechanismen die zu empfehlende Strategie? Insgesamt können vier nichtmarktliche Koordinationsmechanismen unterschieden werden: das Angebot bis zur Sättigung, die Kontingentierung, die Verlosung und das windhundverfahren. 1)

Die Fragestellungen verdeutlichen, daB der ProzeB der Aufgabenerfüllung in der Volkswirtschaft stets durch ein Zusammenspiel dreier Aktionsebenen gekennzeichnet ist, nämlich eines mehr oder minder intensiven Einsatzes staatlicher Instrumente, der Aktivitäten privater und/oder öffentlicher Institutionen und der Anwendung marktlicher oder nichtmarktlicher Koordinationsmechanismen. Demzufolge können öffentliche Eingriffe auf den Interventionsebenen "Instrumente", "Institutionen" und "Koordinationsmechanismen" erfolgen. Allen drei Interventionsebenen steht wiederum jeweils eine Reihe spezifischer Interventionsformen zur Verfügung (siehe übersicht 17).

\subsection{Entscheidungskriterien}

Die Auswahl der geeigneten Interventionsebenen und -formen hat schließlich auf Grundlage von Entscheidungskriterien zu erfolgen, die dann den Charakter hinreichender Bedingungen für eine Korrektur bzw. Ablösung der Marktpreissteuerung besitzen. 2) Stehen - wie in dieser Arbeit - Allokationsgesichtspunkte im Vordergrund, so sind innerhalb des Bündels ökonomischer Entscheidungskriterien in erster Linie Effizienzkriterien von Bedeutung. Das Effizienzkalkuil ist freilich auf eine möglichst

1) Die Beschreibung der unterscheidbaren Koordinationsmechanismen erfolgte im zweiten Teil, Abschnitt III., Punkt 2.1.

2) Erneut ist ausdrücklich zu betonen, daß es sich hier um ökonomische Entscheidungskriterien handelt, die neben einer Vielzahl außerókonomischer Kriterien lediglich die Determinanten der politischen Interventionsentscheidung bilden. Selbst dort, wo ökonomische Aspekte von dominierendem Einfluß sind, dienen sie stets nur der Vorbereitung politischen Handelns: d.h. Sie bieten der Politik zwar Entscheidungshilfe, können sie jedoch niemals ersetzen. 
breite Basis zu stellen. Insbesondere muß über den Aspekt der bloßen (betriebswirtschaftlichen) Kostenminimierung, auf den häufig Privatisierungsuntersuchungen reduziert bleiben, beträchtlich hinausgegangen werden. Notwendig ist eine umfassende Analyse volkswirtschaftlich relevanter Kosten. 1) Als Entscheidungsgrundlage für eine Aufgabenverteilung sind dann folgende gesamtwirtschaftliche Kostenkategorien zu berücksichtigen:

- Interventionskosten. Der Einsatz staatlicher Instrumente zur Korrektur von Funktionsstörungen des Marktpreissystems, die aus gleichem Grunde vollzogenen institutionellen Reorganisationen oder die Ablösung des Marktpreissystems durch einen anderen Koordinationsmechanismus verursachen Interventionskosten. Zu unterscheiden sind die mit öffentlichen Ausgaben einhergehenden direkten Interventionskosten von den als $\mathrm{Re}-$ sultat des öffentlichen Eingriffs im privaten Sektor anfallenden indirekten Interventionskosten. Zu den direkten kosten der Intervention zählen zum einen administrative Kosten wie z.B. die bei Gesetzgebung, Auflagen und Regulierung anfallenden Gemeinkosten sowie die mit der überwachung dieser Maßnahmen verbundenen Kontrollkosten. Sie führen im öffentlichen Sektor $z u$ Personal- und Sachausgaben. Zum anderen werden durch den Einsatz des Instruments "finanzielle Förderung" direkte Interventionskosten verursacht, die sich als Transferausgaben bzw. Subventionen in den öffentlichen Haushalten niederschlagen. Zu den von Unternehmen und Privathaushalten zu tragenden indirekten Interventionskosten gehören jene, die z.B. bei der Einhaltung von Gesetzen oder der Erfuillung von Auflagen anfallen, sowie Kostenbelastungen durch spezifische Steuern oder Abgaben.

1) Nur im Falle einer umfassend angelegten, gesamtwirtschaftlich ausgerichteten und vergleichenden Untersuchung ist es möglich, auf eine separate Ermittlung der volkswirtschaftlichen Nutzen (Erträge) zu verzichten. Sie können dann als Opportunitätskosten interpretiert werden. 
- Transaktionskosten. Sämtliche Kosten der Betreibung eines Koordinationsmechanismus können als Transaktionskosten bezeichnet werden ${ }^{1)}, d . h$. es handelt sich um solche Kosten, die unmittelbar bei der Ressourcenlenkung sowie der Güterbereitstellung anfallen. 2) Nach dem zeitlichen Ablauf des Koordinationsprozesses können Transaktionskosten zur Herstellung einer Kommunikationsbeziehung zwischen den am Koordinationsprozeß beteiligten Akteuren (Informationskosten), Transaktionskosten der Durchführung und der Kontrolle des Koordinationsprozesses unterschieden werden. Diese drei Kategorien der Transaktionskosten ${ }^{3)}$ lassen sich konkreter typologisieren: ${ }^{4}$ )

1. Informationskosten. Sowohl bei den Anbietern als auch bei den Nachfragern von Gütern fallen Informationsbereitstellungs-, -beschaffungs - und -verarbeitungskosten an. Die Nachfrager muissen den Anbietern ihre Präferenzen offenbaren (Informationsbereitstellungskosten), sie müssen über die Gütereigenschaften und Austauschkonditionen Erkundigungen einholen (Informationsbeschaffungskosten) und diese verarbeiten (Informationsverarbeitungskosten). Die Anbie-

1) So bereits K. J. Arrow, The Organization of Economic Activity: Issues Pertinent to the Choice of Market versus Nonmarket Allocation, in: The Analysis and Evaluation of Public Expenditure: The PPB System, Joint Economic Committee, Vol. 1, Washington 1969, S. 48. Zur unterschiedlichen Begriffsbestimmung in der Literatur vgl. E. Bössmann, a.a.0., S. $664 \mathrm{f}$.

2) Von den Transaktionskosten sind somit die mit der Erzeugung eines Gutes oder einer Dienstleistung stets verbundenen Produktionskosten zu trennen. Letztere variieren zwar mit dem jeweiligen Stand der Produktionstechnik, ihre Höhe ist aber unabhängig davon, über welchen Koordinationsmechanismus ein Gut bereitgestellt wird.

3) Dahlmann hält die Berücksichtigung einer einzigen Kategorie, nämlich jener, die die Transaktionskosten allein auf mangelnde Informationen zurückführt, für ausreichend. Tatsächlich können Transaktionskosten zu einem großen Teil, so z.B. auch die Kontrollkosten, als Folge von Informationsdefiziten interpretiert werden. Insgesamt aber dürfte der Ansatz Dahlmanns zu ena sein, zumindest gehen auf diesem Wege wichtige Erklärungsfaktoren - und mithin auch Informationen - verloren; vgl. C. J. Dahlmann, The Problem of Externality, JLawEc 22. Jg. (1979), S. $14 \overline{8}$.

4) Vgl. dazu auch die Aufzählungen bei R. Coase, The Problem of Social Cost, a.a.0., S. 15 und H. Grossekettler, a.a.0., S. 242 sowie die Einteiluna bei E. Bössmann, a.a.0., S. 664 . 
ter wollen ihrerseits Kenntnisse über die Wünche der Nachfrager erlangen, diese verarbeiten und haben Informationen uber ihre Produkte und ihre Austauschkonditionen bereitzuhalten.

2. Durchführungskosten. Zu nennen sind hier zum einen Verhandlungskosten, sofern die Koordination uiber das Marktpreissystem erfolgt. Zum anderen entstehen Kosten der übertragung von Eigentums- oder Nutzungsrechten sowie Ausschlußkosten, falls diejenigen Wirtschaftssubjekte vom Konsum ausgeschlossen werden sollen, die nicht zur Zahlung einer Gegenleistung bereit bzw. aufgrund politischer Entscheidungen nicht zum Empfang bestimmter Güter berechtigt sind.

3. Kontrollkosten. Hierzu zählen sämtliche Kosten der Öberwachung der spezifischen Regeln der Koordinationsmechanismen (Kosten der Sicherstellung der Vertragserfüllung, der Berechtigtenkontrolle etc.) sowie die Kosten der Sanktionsmaßnahmen bei Regelverstößen (z.B. Kosten der Beschreitung des Rechtsweges, der Durchsetzung von Strafmaßnahmen).

- Soziale Kosten sind durch ökonomische Aktivitäten privater oder öffentlicher wirtschaftssubjekte bzw. Institutionen hervorgerufene Belastungen, die von den Verursachern selbst nicht zu tragen sind, sondern auf bestimmbare Dritte oder unbestimmbar auf die Allgemeinheit abgewälzt werden. Kennzeichnend für soziale Kosten ist somit, daß sie zwar gesamtwirtschaftlich existent sind, jedoch keine systematische Berücksichtigung im Rechensystem eines Koordinationsmechanismus finden. Gleichguiltig, über welchen Koordinationsmechanismus die Bereitstellung der Giuter und Dienstleistungen erfolgt, ist stets mit dem Auftreten sozialer Kosten zu rechnen, allerdings sind Art und Höhe von der Ausgestaltung des Koordinationsverfahrens abhängig. Die Nichtbeachtung der persönlichen Präferenzen und damit Einschränkungen der individuellen Entscheidungsfreiheit der Wirtschaftssubjekte erweisen sich dagegen als soziale kosten, die für Koordinationsmechanismen wie Kontingentierung und Angebot bis zur Sättigung mit Benutzerzwang bezeichnend 
sind. Auch öffentliche Interventionen zur Verbesserung der Funktionsweise eines bestehenden Koordinationsmechanismus können soziale Kosten verursachen, falls z. B. mit einer Intervention die allokative Zielsetzung "Verbesserung des Wettbewerbs" zwar erreicht, aber das Allokationsziel "technischer Fortschritt" nachhaltig verletzt wird.

Die Kostenkategorien treten nicht unabhängig voneinander auf, sondern sie stehen in einem engen Zusammenhang. So kann z.B. die Einschränkung von Umweltschäden und damit die Senkung der sozialen Kosten u.a. durch finanzielle Anreize oder kontrollintensive Auflagen für umweltverschmutzende Unternehmen erreicht werden. In beiden Fällen entstehen Interventionskosten. Umweltschäden können aber auch durch eine exaktere Definition der Verfügungsrechte reduziert werden. In diesem Fall geht mit der Senkung der sozialen Kosten eine Erhöhung der Transaktionskosten einher.

Als Entscheidungsgrundlage für eine ökonomisch zweckmäßiae Aufgabenverteilung in der Marktwirtschaft sind somit stets sämtliche gesamtwirtschaftlich relevanten Kostenkategorien zu berücksichtigen. Bisher wurde bei Effizienzvergleichen alternativer Formen der Güterbereitstellung das Augenmerk allerdings uberwiegend auf die direkten Interventionskosten gelegt und nur hin und wieder der versuch gemacht, auch die indirekten Interventionskosten zu erfassen. Fast völlig vernachlässigt wurde dagegen bisher die Analyse der Transaktionskosten. Und schließlich ist zwar allgemein akzeptiert, daß der Marktpreismechanismus soziale Kosten erzeugt, es mangelt aber bisher an qualitativ vergleichbaren Untersuchungen, die sich mit den sozialen Kosten nichtmarktlicher Koordinationsmechanismen beschäftigen.

Die relative Leichtigkeit, mit der die Entscheidungskriterien formuliert werden können, steht in einem auffälligen Mißverhältnis zu den gravierenden Schwierigkeiten, die mit einer praktischen Anwendung der Kriterien verbunden sind. "Wenn die- 
se Schwierigkeiten nicht erkannt und angemessen berücksichtigt und Schritte zu ihrer überwindung getan werden, bleibt auch der durchdachteste Kriterienkatalog am Ende praktisch nutzlos."1)

Nur in Ausnahmefällen sind alle relevanten Kostengrößen eindeutig meBbar. KASTLI und SAURER stellen fest, die Obertragung einer Aufgabe an den Staat sei aus volkswirtschaftiicher Sicht nur dann zweckmäßig, wenn die Erträge aus der Korrektur eines Marktversagens die durch den Staatseingriff ausgelösten Kosten übersteigen, die Aufgabenverteilung sei mithin als optimierungsproblem im Sinne der Kosten-Ertrags-Analyse zu begreifen. 2) Dem ist im Prinzip zuzustimmen, allerdings muß einschränkend angemerkt werden, daß sich solche Optimierungsrechnungen bisher als untauglich erwiesen haben, sämtliche Ertrags- und Kostenbestandteile, die bei dem Einsatz staatlicher Instrumente oder bei einem Wechsel des Koordinationsmechanismus zu berücksichtigen sind, vollständig zu erfassen. Vor allem im Hinblick auf die Transaktionskosten und die sozialen Kosten ergeben sich elementare Meßprobleme. Die vorhandenen Meßprobleme dürfen andererseits jedoch nicht als Alibi für den Verzicht auf umfassende Vergleichsanalysen herangezogen werden. Vielmehr gilt es, weiterhin an einer Verbesserung der Meßtechniken zu arbeiten.

Die Höhe der gesamtwirtschaftlichen Kosten ist nicht nur von der Art der Aufgabenerfüllung, sondern auch vom Umfang bzw. von der Intensität der Aufgabenwahrnehmung abhängig. Für die Kriterienbildung ließe sich dieses Problem lösen, indem die Entscheidung über eine Aufgabenverteilung stets unter der Prämisse eines unveränderten Niveaus der Aufgabenerfïllung getroffen würde. Jedoch kann anhand mehrerer Beurteilungskriterien ge-

1) R. Mayntz, Sachverständigenanhörung ..., a.a.0., S. 41.

2) $\mathrm{Vgl}$. R. Kästli und $P$. Saurer, Aufgaben und Funktionsweise des Staates aus ökonomischer Sicht, SZVS 114. Jg. (1978), S. $62 \mathrm{f}$. 
zeigt werden, daß bei einer zu geringen Marktnachfrage oder einem nicht ausreichend entwickelten Marktangebot Interventionen in den Marktpreismechanismus gerade unter der ausdrücklichen Zielsetzung einer Niveauverbesserung angestrebt werden.

Auch eine sorgfältige und systematische Erarbeitung von Beurteilungs- und Entscheidungskriterien für eine Aufgabenverteilung läßt nur Tendenzaussagen zu, da die besonderen Bedingungen des konkreten Einzelfalls keine Berücksichtigung finden können. Aus diesem Grunde bleiben ergänzende Einzelfallanalysen unerläßlich, welche die aus allgemeineren Zusammenhängen abgeleiteten Ergebnisse u.U. revidieren können.

\section{Kriterien für ein allokatives Marktversagen}

1. Marktversagen auf der Angebotsseite

1.1. Monopolistisches Unternehmensverhalten

Eines der am häufigsten genannten Argumente für ein Versagen des Marktes ergibt sich aus monopolistischen Elementen im Marktpreissystem, d.h. konkret aus dem Bestreben auf Seiten der Unternehmen, sich der Konkurrenzsituation durch den Aufbau einer marktbeherrschenden Stellung weitgehend oder vollkommen zu entziehen.

Eine zweckmäßige Kennzeichnung dieser Monopolelemente sollte über die Systematisierung der Marktformen anhand der zahlenmäßigen Besetzung der Marktseiten, wie sie VON STACKELBERG vorg̣enommen hat ${ }^{1)}$, hinausgehen. Bei diesem Ansatz wird die atomistische Konkurrenz durch viele kleine Produzenten, das 01 igopol durch einige wenige Unternehmer und das Monopol schließlich durch den Fall des Alleinanbieters umschrieben. Wesentlich aussagekräftiger ist eine Charakterisierung monopolistischer Elemente, welche die Verhaltensweisen der Unternehmen in den Vor-

1) Vgl. H. von Stackelberg, Grundlagen der theoretischen Volkswirtschaftslehre, 2. AufT., Tübingen und Zürich 1951, S. $231 \mathrm{ff}$. 
dergrund rückt. Für den empirischen Nachweis monopolistischer Verhaltensweisen bedarf es dann der Berücksichtigung mehrerer Bestimmungsfaktoren, zu denen neben der Zahl der Anbieter insbesondere auch Größe und Marktanteile der Unternehmen, Kostenfunktionen, Preiselastizitäten, Marktzugang und Markttransparenz gehören. Als Resultat konkreter Ausprägungen der Einflußgrößen ermöglicht monopolistisches Unternehmensverhalten dem Anbieter, seine Aktionsparameter - z.B. Preise oder Mengen, Produktqualität, Risikoneigung, Innovationsbereitschaft - zu variieren, ohne daß er eine seine Position gefährdende Reaktion konkurrierender Anbieter zu befürchten hat. 1)

Wie sich wettbewerbsbeschränkende Verhaltensweisen auf die Höhe der Preise und auf die Absatzmengen auswirken, das $1 a ̈ B t ~ s i c h$ bereits anhand eines einfachen Effizienzvergleichs des Allokationsprozesses auf einem Konkurrenzmarkt (Modell der vollständigen Konkurrenz) und einem Monopolmarkt (Modell des reinen Monopols) verdeutlichen. Ist ein Unternehmer bestrebt, die für ihn gewinnmaximale Produktionsmenge abzusetzen, so wird dieses Ziel in einem Konkurrenzmarkt dann erreicht, wenn die letzte von ihm verkaufte Einheit keinen Gewinn mehr erwirtschaftet, oder anders ausgedrückt, wenn die zur Gewährleistung der effizienten Allokation notwendige Regel "Grenzkosten gleich Preis" eingehalten wird. In der Graphik 1 ist dies bei dem Preis $P_{w}$ der Fall, bei dem sich eine Absatzmenge $x_{w}$ ergibt. Aus dem gewinnmaximierenden Verhalten des Monopolisten resultiert dagegen ein Preis $P_{m}$, determiniert durch den Schnittpunkt von Grenzkostenkurve $K^{\prime}$ und Grenzerlöskurve $E^{\prime}$ sowie dem Verlauf der Nachfragekurve $N$, der beträchtlich oberhalb des Wettbewerbspreises $P_{W}$ liegen kann. ${ }^{2}$ )

1) Die Unterschiede zum 01 igopol sind dann nur gradueller Art. Verändert der 01 igopolist seine Aktionsparameter, so hat er zu erwartende Reaktionen der übrigen 01 igopoliston in seine überlegungen aufzunehmen.

2) Die Differenz zwischen Monopol- und Wettbewerbspreis häng̣t neben den Verläufen der Grenzkosten- und Grenzerlöskurven auch von der Elastizität der Nachfragekurve ab. Man spricht in diesem Zusammenhang auch von einer konjekturalen Preis-Absatz-Funktion, welche die vom Monopol isten erwarteten Reaktionen der Nachfrager auf Preisänderungen zum Ausdruck bringt, d.h. sie gibt Auskunft über die Mennen, die der Monopolist zu alternativen von ihm fixierten Preisen absetzen kann. 
Mit diesem Monopolpreis $P_{m}$ korrespondiert eine Ausbringungsmenge $x_{m}$, die geringer ausfällt als die allokationseffiziente Absatzmenge $x_{w}$.

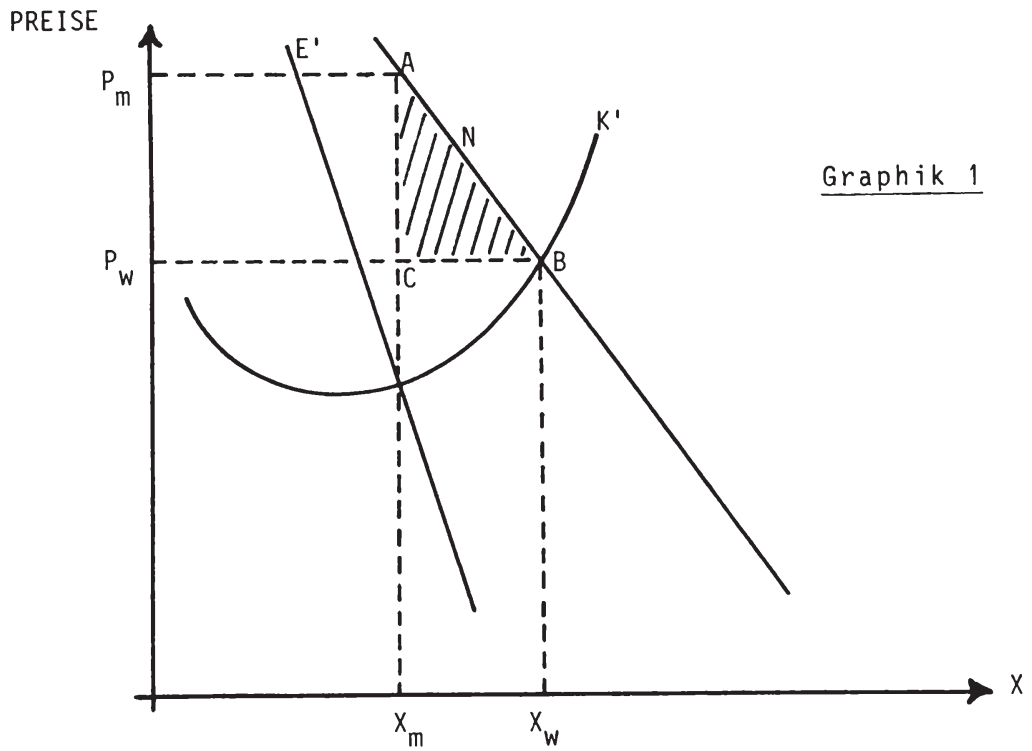

MENGE

Ebenso wie über den Tatbestand Einigkeit herrscht, daß die Marktwirtschaft seit Beginn der Industrialisierung zu Monopolbildungen neigt ${ }^{1)}$, scheinen auch die unmittelbaren Kosequenzen monopolistischen Unternehmensverhaltens auf der Hand zu liegen: Die Preise sind höher, die Absatzmengen niedriger als in der Konkurrenzsituation. Das marktwirtschaftliche system funktioniert mithin nicht mehr störungsfrei, d.h. es tritt eine Feh1allokation der Ressourcen ein, sofern Interventionen in das

1) Bereits Adam Smith beklagt den Monopolgeist der Kaufleute und Unternehmer: "Geschäftsleute des gleichen Gewerbes kommen selten, selbst zu Festen und zur Zerstreuung, zusammen, ohne daß das Gespräch in eine Verschwörung gegen die offentlichkeit endet oder irgende in Plan ausgeheckt wird, wie man die Preise erhöhen kann." A. Smith, a.a.0., S. 112; vgl. auch S. 407. 
Marktpreissystem unterbleiben. Im Hinblick auf den zuletzt genannten Punkt ändert sich jedoch das Räsonnement, sobald das Modell der vollständigen Konkurrenz als Referenzsystem aufgegeben wird. Zumindest sind nunmehr die ökonomischen Implikationen monopolistischer Verhaltensweisen in einer Marktwirtschaft und folglich auch die Fragen nach dem 0 b und Wie öffentlicher Interventionen höchst umstritten.

Aus der Kritik am Modell der vollständigen Konkurrenz sind mit dem Konzept des funktionsfähigen Wettbewerbs (workable competition) einerseits und dem Konzept der Wettbewerbsfreiheit andererseits zwei unterschiedliche Grundkonzeptionen hervorgegangen, die zu einer seit Jahrzehnten im In- und Ausland andauernden kontroversen wettbewerbstheoretischen Diskussion gefuihrt haben. 1 )

Der workable competition-Ansatz hat seine Wurzeln in dem von SCHUMPETER propagierten Pionierunternehmer, der durch seine Innovationsbereitschaft erst technisch-wirtschaftlichen Fortschritt ${ }^{2}$ ermöglicht und damit zum Garanten eines dynamischen Wettbewerbs wird. Als kräftigsten Motor für Fortschritt und wirtschaftliches Wachstum weist er dabei den Großunternehmern eine herausragende Stellung zu. Der Preis sind freilich zumindest temporär auftretende Monopolstellungen, da die stets mit Risiken behafteten Innovationen nur vorgenommen werden, falls dem Pionierunternehmer ein zeitlich befristeter Schutz vor ihn imitierenden Konkurrenzanbietern gewährt wird. ${ }^{3)}$ Für CLARK

1) Gegenüberstellungen der wesentlichen Grundaussagen beider Konzepte finden sich $u . a$. bei $H$. Cox und $H$. Hübener, Wettbewerb. Eine Einführuna in die Wettbewerbstheorie und Wettbewerbspolitik, in: Handbuch des Wettbewerbs, hrsg. von H. Cox, U. Jens und K. Markert, München 1981, S. $1 \mathrm{ff}$. sowie bei H. Bartling, Leitbilder der Wettbewerbspolitik, München 1980, S. $20 \mathrm{ff}$.

2) Den technisch-wirtschaftlichen Fortschritt bezeichnet Schumpeter als einen "Prozeß der schöpferischen Zerstöruna", "der unaufhörlich die Wirtschaftsstruktur von innen heraus revolutioniert, unaufhörlich die alte Struktur zerstört und unaufhörlich eine neue schafft". (Unterstreichung im Original kursiv). J. Schumpeter, a.a.0., S. $137 \mathrm{f}$.

3) Vgl. J. Schumpeter, a.a.0., S. $171 \mathrm{ff}$. 
stellt die These SCHUMETERS, wirtschaftlicher Fortschritt sei unabdingbar mit monopolistischen Strukturen verbunden, ein Dilemma dar, das eine Entscheidung zugunsten eines der beiden wirtschaftspolitischen Ziele "wirtschaftlicher Fortschritt" und "vollkommener Wettbewerb" erfordert. Er lehnt deshalb das Modell der vollständigen Konkurrenz als Leitbild ab, d.h. Unvollkommenheiten des Marktpreissystems werden nicht mehr als unerwünschte Abweichungen von einem Idealzustand beklagt, sondern als Voraussetzungen für einen dynamischen Wettbewerbsprozeß ausdrücklich begrüßt. Er plädiert für ein Wettbewerbskonzept, welches unter den tatsächlichen Marktbedingungen auch realisierungsfähig (workable) ist. Der Wettbewerb verliert damit die Eigenschaft eines eigenständigen wirtschaftspolitischen. Zieles und erhält statt dessen funktionalen charakter, wird mithin zu einem wirtschaftspolitischen Instrument zur Erfüllung gesamtwirtschaftlicher Ziele. ${ }^{1}$ ) Eine Weiterentwicklung des Konzepts eines funktionsfähigen Wettbewerbs nimmt KANTZENBACH vor. Er analysiert die Bedingungen für eine optimale Wettbewerbsintensität, d.h. für Wettbewerbs prozesse, die zugleich sowohl eine hohe Innovationsbereitschaft der Unternehmer als auch eine flexible Faktorallokation gewährleisten und damit ein Höchstmaß an gesamtwirtschaftlichen Antriebs- und Steuerungsfunktionen erfüllen. Sein Ergebnis, eine optimale Wettbewerbsintensität sei am ehesten im Bereich weiter 01 igopole mit mäßiger Produktdifferenzierung und unvollkommener Markttransparenz zu erreichen, deckt sich mit den Schlußfolgerungen CLARKS. 2 )

Im Gegensatz zum Konzept des funktionsfähigen Wettbewerbs verneint das von HOPPMANN formulierte, in der klassischen und neoliberalen Tradition stehende Wettbewerbsfreiheitskonzept den Zielkonflikt zwischen Freiheit des wettbewerbs und wirtschaftlicheni Fortschritt. Statt dessen wird ein freier, unaehinderter

1) Vgl. J. M. Clark, Competition as a Dynamic Process, Washington, D.C. 1961 sowie H. Cox und H. Hübener, a.a.0., S. $14 \mathrm{ff}$.

2) Vgl. E. Kantzenbach und H. H. Kallfass, Das Konzept des funktionsfähigen Wettbewerbs - workable competition -, in: Handbuch des Wettbewerbs, hrsg. von H. Cox, U. Jens und K. Markert, München 1981, S. $103 \mathrm{ff}$. 
Wettbewerb als Voraussetzung für die Erreichung ökonomisch vorteilhafter Marktergebnisse angesehen. 1)

Im Hinblick auf die unter Allokationsaspekten zweckmäßige Aufgabenbestimmung und -verteilung geben die beiden Grundkonzeptionen deshalb auch erwartungsgemäß äußerst divergierende wettbewerbspolitische Empfehlungen.

Das Wettbewerbsfreiheitskonzept formuliert als öffentliche Aufgabe die "Beseitigung monopolistischen Unternehmensverhaltens durch Per-se-Verhaltensverbote" 2 ), d.h. es erfolgt eine Verknüpfung von Aufgabenbestimmung mit der Art und Weise der Aufgabenerfüllung. Exakter ausgedrückt: Da monopolistische Verhaltensweisen bereits von vornherein einen Interventionsbedarf erzeugen und die adäquate Interventionsform ebenfalls bereits ex ante festgelegt ist, stellt in diesem Konzept "monopolistisches Unternehmensverhalten" nicht lediglich ein (notwendiges) Beurteilungskriterium dar, sondern es erhält gleichzeitiog den Rang eines (hinreichenden) Entscheidungskriteriums. In dieser Verknüpfung von Aufgabenbestimmung und -erfüllung liegt u. E; die entscheidende Schwäche des Wettbewerbsfreiheitskonzepts ${ }^{3)}$, denn es versperrt den Blick auf andere, eventuell besser geeignete Formen der Aufgabenwahrnehmung und verhindert damit das Denken in Alternativen. ${ }^{4)}$ Darüber hinaus ist aber auch die restriktive wettbewerbspolitische Forderung nach Beseitigung jeglicher Monopolmacht kritisch zu vermerken. Mit ihr wird weder die Existenz positiver Effekte der Monopolisierung für möglich gehalten noch berücksichtigt, daß mit jedem Eingriff in den Markt-

1) Vgl. H. Cox und H. Hübener, a.a.0., S. $28 \mathrm{ff}$.

2) Unter Per-se-Verhaltensverboten ist ein durch Rechtsnormen eindeutin festgelegtes System allgemein gültiger Spielregeln zu verstehen; vgl. dazu H. Bartling, a.a.0., S. $47 \mathrm{f}$.

3) Die Verknüpfung von Aufgabenbestimmung und -erfüllung wurde bereits im ersten Teil, Abschnitt I. 2. allgemein kritisiert.

4) Die von Bartling gegen das Wettbewerbsfreiheitskonzept hervorgebrachten Kritikpunkte d) und e) sind nur Ausflüsse der Verknüpfung von Aufgabenbestimmung und -erfüllung; vgl. H. Bartling, a.a.0., S. $54 \mathrm{ff}$. 
preismechanismus zumindest Interventionskosten, im Regelfall aber auch soziale Kosten anfallen, die das ökonomische Kalkül in bezug auf die Interventionsentscheidung beeinflussen. 1)

Die Vertreter des Konzepts des funktionsfähigen Wettbewerbs sehen die vorrangige öffentliche Aufgabe darin, "das Ausmaß der Wettbewerbsbeschränkung so zu begrenzen, daß die Funktionsfähiakeit des Wettbewerbs weitestmöglich erhalten bleibt."2) sie schlagen eine Reihe grundsätzlich denkbarer Interventionsformen zur Erfüllung dieser Aufgabe vor, die unter dem Stichwort "staatliche Marktgestaltungspolitik"3) zusammengefaßt werden. Damit fallen die wirtschaftspolitischen Handlungsanweisungen des Kantzenbach-Konzepts in zweifacher Hinsicht differenzierter aus als die des Wettbewerbsfreiheitskonzepts. Zum einen wird die restriktive Handhabung monopolistischer Verhaltensweisen insofern aufaegeben, daß der vermutete Zusammenhang zwischen Konzentrationstendenzen und wirtschaftich-technischem Fortschritt zu der Empfehlung führt, Wettbewerbsbeschränkungen in gewissem Umfange zuzulassen. Zum anderen wird die Verknüpfung von Aufgabenbestimmung und -erfüllung gelockert, indem monopolistisches Unternehmensverhalten zunächst nur als notwendiges Beurteilungskriterium für die Feststellung eines Interventionsbedarfes angesehen wird. Die Beantwortung der Frage, ob und gegebenenfalls in welcher Form zu intervenieren ist, eraibt sich hingegen erst als Resultat der Anwendung hinreichender Entscheidungskriterien, nämlich der Nichterfüllung von workable-Normen.

Folgendes ist allerdings hier kritisch anzumerken.

Erstens: Zwar wird mit der Formulierung "optimale Wettbewerbsintensität als öffentliche Aufgabe" von der Ausrichtung der Politik an das unrealistische Modell der vollständigen Konkurrenz Abschied genommen, dennoch gelingt keine vollständige überwindung des "Nirwana"-Ansatzes, erfordert doch die anvisierte

1) Weitere Kritikpunkte bei H. Bartling, a.a.0., S. $49 \mathrm{ff}$.

2) E. Kantzenbach, Die Funktionsfähigkeit des Wettbewerbs, Göttingen 1966 , 5. 143 (Unterstreichung im Original hervorgehoben).

3) Vgl. H. Cox und H. Hübener, a.a.0., S. 31. 
optimale Wettbewerbsintensität stets dann öffentliche Eingriffe in den Marktpreismechanismus, wenn Abweichungen von dem Optimum feststellbar sind. Wie beim Wettbewerbsfreiheitskonzept wird damit vernachlässigt, daß zur Beseitigung der durch Monopolisierung entstandenen Fehlallokation Interventionskosten anfallen und darüber hinaus ständige und umfangreiche Eingriffe in das Marktpreissystem zur Wiederherstellung des "Optimalwettbewerbs" ebenfalls soziale Kosten für die Gesamtwirtschaft verursachen können.

Zweitens: Infolge der einseitigen Orientierung an einer optimalen Wettbewerbsintensität wird die Wahl der Interventionsebenen von vornherein eingeschränkt. Eine Alternative zum Wettbewerb kommt nicht in Betracht, d.h. die Möglichkeit der Ressourcensteuerung über einen anderen Koordinationsmechanismus als das Marktpreissystem wird zwangsläufig ausgeblendet. ${ }^{1}$ )

Sollen die für eine Aufgabenverteilung entwickelten Kriterien dem Anspruch ökonomisch rationaler Entscheidungshilfe für die Wettbewerbspolitik genügen, so ist die Untersuchung in zwei Richtungen auszuweiten.

Zunächst ist eine systematische Erfassung der wettbewerbspolitisch relevanten Interventionsformen - getrennt nach den Ebenen Instrumente, Institutionen und Koordinationsmechanismen erforderlich. Einige der wichtigsten Eingriffsmöglichkeiten, geordnet nach Interventionsformen und -ebenen sind in obersicht 18 aufgeführ. ${ }^{2)}$

1) Weitere Kritikpunkte nennt H. Bartling, a.a.0., S. $36 \mathrm{ff}$.

2) Eine äußerst differenzierte Darstellung der wettbewerbspolitischen Instrumente und Institutionen nimmt Bartling vor. Er unterscheidet eine ursachenadäquate und eine neutral isierende Wettbewerbspolitik und untersucht anschließend drei Fälle, nämlich (a) den Wettbewerbsschutz durch Verbote wettbewerbsbeschränkender Verhaltensweisen, (b) die Mißbrauchsaufsicht als neutralisierende Politik bei marktstrukturell bedingten Wettbewerbsbeschränkungen sowie (c) die Wettbewerbsförderung als ursachenadäquate Politik gegen verfestigte marktstrukturell bedingte Wettbewerbsbeschränkungen; vgl. H. Bartling, a.a.0., S. $59 \mathrm{ff}$. 
Übersicht 18: Interventionsebenen und -formen be $i$ monopolistischem Unternehmensverhalten

\begin{tabular}{|c|c|}
\hline $\begin{array}{l}\text { INTERVENT IONSEBENE } \\
\text { "INSTRUMENTE" }\end{array}$ & $\begin{array}{l}\text { Informationsbereitstellung: } \\
\text { - Monopol- und Kartellregistrierung } \\
\text { - Aufklärung und Beratung potentieller Anbieter } \\
\text { - Appelle in Form gezielter Aufforderungen und } \\
\text { Warnungen an den Wettbewerb beschränkende } \\
\text { Unternehmen (moralische Oberredung) } \\
\text { Finanzielle Förderung/Belastung: } \\
\text { - Zusatzbesteuerung von Monopolgewinnen } \\
\text { - Verbesserung der Markteintrittschancen z.B. } \\
\text { durch Subventionen, Bereitstellung von Risiko- } \\
\text { kapital, Steuernachlässen (international auch } \\
\text { durch Senkung von Zöllen) } \\
\text { - Förderung der Substitutionskonkurrenz } \\
\text { Staatliche Auftragsvergabe: } \\
\text { - zur Förderung des Markteintritts } \\
\text { - zur Förderung der Substitutionskonkurrenz } \\
\text { Auflagen, Regulierung: } \\
\text { - Melde- und Genehmigungspflicht bei Unterneh- } \\
\text { menszusammenschlüssen } \\
\text { - Preis-/Mengenregulierung } \\
\text { - Qualitäts- und Konditionsregulierung } \\
\text { Ge- und Verbote: } \\
\text { - Verbot des unlauteren Wettbewerbs } \\
\text { - Verbot wettbewerbsbeschränkender Verein- } \\
\text { barungen } \\
\text { - Mißbrauchsaufsicht }\end{array}$ \\
\hline $\begin{array}{l}\text { INTERVENT IONSEBENE } \\
\text { "INSTITUTIONEN" }\end{array}$ & $\begin{array}{l}\text { Gemischtwirtschaftliche Unternehmen } \\
\text { (öffentliche Beteiligung) } \\
\text { offentliche Unternehmen } \\
\text { - Offentliche Unternehmen als zusätzliche Anbieter } \\
\text { - Verstaatlichung }\end{array}$ \\
\hline $\begin{array}{l}\text { INTERVENT IONSEBENE } \\
\text { "KOORDINATIONS- } \\
\text { MECHANISMEN" }\end{array}$ & $\begin{array}{l}\text { Kontingentierung } \\
\text { Angebot bis zur Sättigung }\end{array}$ \\
\hline
\end{tabular}


Darüber hinaus sind bei der Wahl der zweckmäßigen Interventionsform Entscheidungskriterien anzulegen, in denen die unterschiedlich hohen Kosten der wirtschaftspolitischen Gestaltungsalternativen eingehen und der gesamtwirtschaftlichen (sozialen) Kosten monopolistischen Unternehmensverhaltens gegenübergestellt werden.

Die durch Monopolisierung verursachten sozialen Kosten lassen sich theoretisch recht einfach anhand des in Graphik 1 dargestellten Effizienzvergleichs zwischen dem AllokationsprozeB auf einem Konkurrenz- und einem Monopolmarkt bestimmen. Die monopolistische Preisfixierung führt verglichen mit der Preisbildung bei vollständiger Konkurrenz zu einem Monopolgewinn entsprechend der Fläche $P_{m} A C P_{W}$, dem in gleicher Höhe Einkommensverluste der Konsumenten gegenüberstehen. Da Verluste einer Gruppe durch Gewinne einer anderen Gruppe kompensiert werden, entstehen dadurch noch keine allokativ bedingten Wohlfahrtsverluste für die Gesamtwirtschaft. Die Preiserhöhurg des Monopolisten hat jedoch auch eine Verringerung der Absatzmenge um $x_{w}-x_{m}$ zur Folge. In Höhe dieser Absatzreduzierung wird eine Fehlallokation von Ressourcen verursacht, verbunden mit einem Effizienzverlust für die Volkswirtschaft insgesamt, die als soziale Kosten der Monopolisierung bezeichnet werden können. Ihr Ausmaß wird in der Graphik durch das schraffierte Dreieck $A B C$ wiedergegeben. 1)

Die auf diesem Wege vorgenommene Ableitung sozialer Kosten monopolistischen Unternehmensverhaltens harmoniert jedoch ausgesprochen schlecht mit den Gegebenheiten der Realität, wird doch erneut das statische Modell der vollständigen Konkurrenz als

1) üblicherweise bedient man sich zur Darstellung der infolge Monopolisierung verursachten sozialen Kosten des Konzepts der Konsumentenrente. Aufgrund der monopolistischen Preisfixierung entsteht ein Verlust an Konsumentenrente ( $F$ läche $P_{m} A B P_{w}$ ), der teilweise durch die vom Monopolisten erzielte Produzentenrente ausgeglichen wird (Fläche $P_{m} A C P_{w}$ ). Es verbleibt folglich ein Nettoverlust an Konsumentenrente (deadweight loss), welcher der Fläche des Dreiecks ABC entspricht. 
Referenzfall herangezogen und damit lediglich die Erfassung statistischer Wohlfahrtsverluste ermöglicht. Es ist deshalb zumindest bemerkenswert, daß auch neuere empirische Untersuchungen auf diesem Gebiet sich immer noch mit Akribie der verfeinerten Messung der durch Monopolmacht verursachten statischen Effizienzverluste widmen ${ }^{1)}$, statt sich intensiver den Möglichkeiten der Berücksichtigung dynamischer Effizienzaspekte, d.h. den ökonomischen Voraussetzungen für technologischen Fortschritt sowie den davon ausgehenden Einflüssen auf die gesamtwirtschaftliche Allokation zuzuwenden. 2)

Wie gezeigt, trägt das Konzept des funktionsfähiaen Wettbewerbs diesen dynamischen Aspekten Rechnung, indem es ausgehend von der Schumpeterschen These, technischer Fortschritt als Voraussetzung für wirtschaftliches Wachstum erfordere zumindest temporäre Monopolstellungen, die optimale Wettbewerbsintensität als Kompromiß zwischen den beiden Extremen Monopol und polypolistischer Markt begreift. Gemäß diesen Vorstellungen würde nicht nur monopolistisches Unternehmensverhalten soziale Kosten hervorrufen, sondern auch der polypolistische Wettbewerb hätte aufgrund des mangelnden technischen Fortschritts Effizienzverluste und mithin eine Belastung der Volkswirtschaft mit sozialen Kosten zur Folge.

Trotz der erheblichen Skepsis, die bereits den Versuchen, die statischen Effizienzverluste der Monopolisierung zu messen, entgegengebracht werden muß und die sich noch weiter verstärkt, sobald die restriktiven Annahmen der statischen Analyse zuqun-

1) Für den angelsächsischen Sprachraum sind hier insbesondere Cowling und Mueller zu nennen, die für die Industrie der USA und Großbritanniens firmenspezifisch verursachte Wohlfahrtsverluste berechnen; vgl. K. Cowling und $D$. C. Mueller, The Social Costs of Monopoly Power, in: The Economic Journal, 88. Jg. (1978), S. 727 ff.; eine Untersuchung für die Bundesrepublik unternimmt erstmalig I. Böbel, Wohlfahrtsverluste durch Marktmacht: Eine Untersuchung für den Bereich der Industrie der Bundesrepublik Deutschland, Jb.Nat.Stat. Bd. 197 (1982), S. 509 ff.

2) $\mathrm{Vgl}$. dazu auch die kritische Auseinandersetzung mit dem von Cowling und Mueller zugrundegelegten theoretischen Rahmen bei S. C. Littlechild, Misleading Calculations of the Social Costs of Monopoty Power, in: The Economic Journal, 91. Jg. (1981), S. $348 \mathrm{ff}$. 
sten einer dynamischen Betrachtungsweise aufgehoben werden, 1 äßt sich der Sachverhalt doch tendenziell mit Hilfe einer Sozialkostenkurve in Abhängigkeit des Monopolisierungsgrades darstellen (Kurve $A$ in Graphik 2). Im extremen Monopolfall entstehen relativ hohe soziale Kosten, mit Intensivierung des Wettbewerbs sinken sie ständig, bis ein Minimum erreicht ist. Analog zum Konzept des funktionsfähigen Wettbewerbs ist im Minimum der Sozialkostenkurve die optimale Wettbewerbsintensität bzw. der optimale Monopolisierungsgrad Mopt realisiert. Nimmt das Ausmaß des Wettbewerbs weiter $z u$, so geht damit ein erneuter Anstieg der Sozialkostenkurve einher. 1 )

Liegt monopolistisches Unternehmensverhalten vor, so führt der Einsatz der in obersicht 18 aufgeführten statlichen Interventionsformen zu einer Intensivierung des Wettbewerbs und damit einhergehend zu einer Reduzierung der gesellschaftichen Kosten gemäß des Verlaufs der Sozialkostenkurve. Im Punkte Mopt wäre demnach auch der optimale Interventionsgrad erreicht, allerdings nur unter der illusionären Annahme, staatliche Eingriffe in das Marktpreissystem erfolgten völlig "kostenlos". Der Sozialkostenkurve ist daher eine Interventionskostenkurve $B$ gegenüberzustellen, die der Tatsache Rechnung trägt, daß jeder Einsatz staatlicher Instrumente oder jede institutionelle $\mathrm{Re}-$ organisation zur Senkung der durch Monopolmacht bedingten sozialen Kosten ebenfalls Kosten verursacht. Wie bereits ausgefuhrt, sind dabei die direkten Interventionskosten wie z.B. finanzielle Förderungsmaßnahmen zur Verbesserung der Markteintrittschancen potentieller Konkurrenten oder administrative Kosten der Regulierung - Kosten also, die bei den öffentlichen Haushalten zu Ausgaben führen - von den indirekten Interven-

1) Der überproportionale Anstieg der Sozialkostenkurve bei zunehmendem Monopolisierungsgrad ist mit der These Posners (vor ihm bereits Tullock), daß bei erhöhter Marktmacht verstärkte Anstrengungen seitens des Monopolisten zur Absicherung seiner Position unternommen werden, zu bearünden. Damit geht eine zusätzliche, weitere soziale Kosten verursachende Bindung von Ressourcen einher. Im einzelnen dazu R. A. Posner, The Social Costs of Monopoly and Regulation, JPolEc 83. Jg. (1975), S. $807 \mathrm{ff}$. 


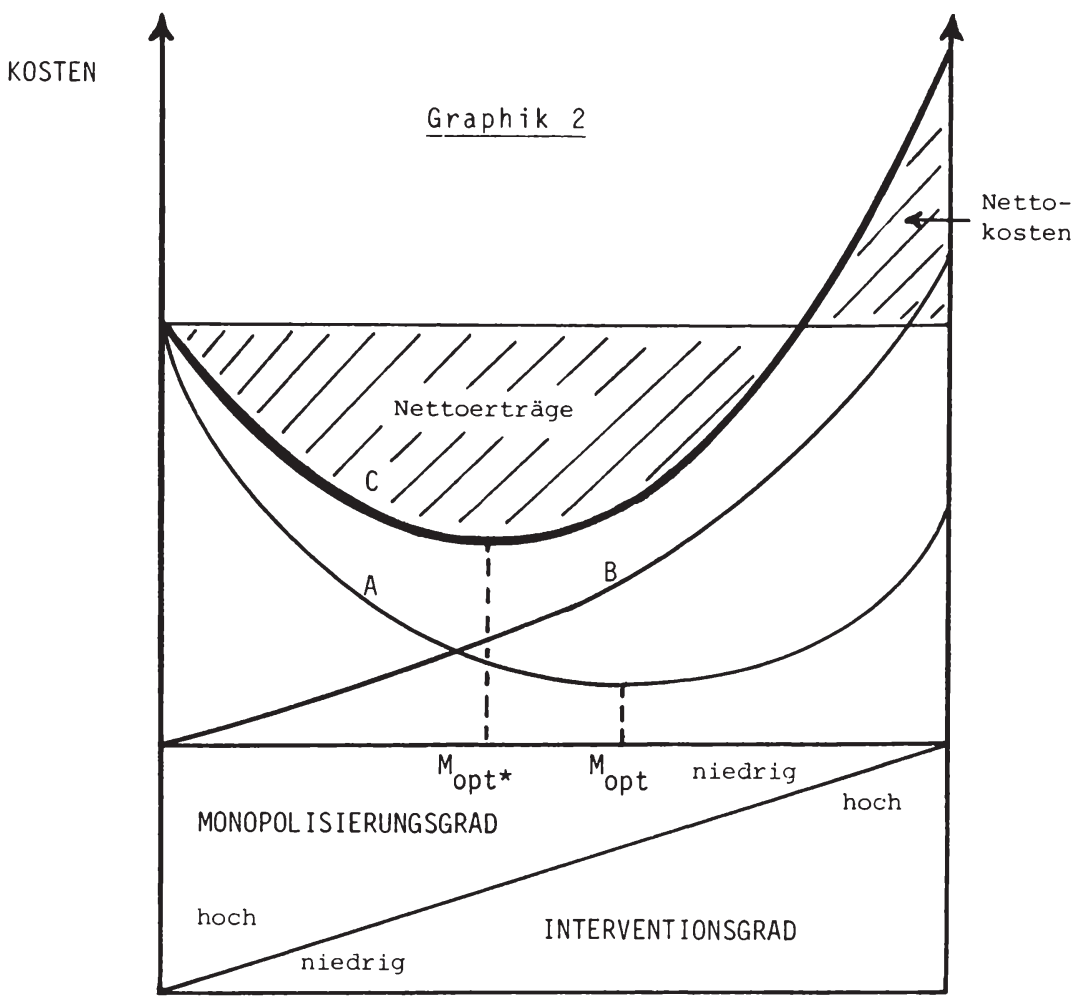

tionskosten zu unterscheiden. Letztere fallen als Resultat der öffentlichen Eingriffe bei den sich monopolistisch verhaltenden Unternehmen an (z.B. Erfüllung von Auflagen, Einhaltung von Gesetzen, erhöhte Qualitätsforderungen, zunehmender Verwaltungsaufwand durch Erlasse, Vorlage von Berichten etc.). Darüber hinaus können auch soziale Kosten der Intervention auftreten, falls mit zunehmenden Eingriffen in das Marktpreissystem Unternehmen mehr und mehr ihre Anstrengungen darauf konzentrieren, die statlichen Maßnahmen zu umgehen bzw. in ihrem Sinne zu beeinflussen. 
Es erscheint plausibel, daß mit zunehmenden Bemühungen, den Grad der Monopolisierung zu senken, die Kosten der statlichen Intervention ansteigen werden. Je intensiver dabei die Wiederherstellung polypolistischer Marktstrukturen angestrebt wird, desto eher ist überdies mit einem überproportionalen Anstieg der indirekten und wohl auch der sozialen Kosten einer Intervention zu rechnen.

Durch vertikale Addition der Sozialkosten- und der Interventionskostenkurve erhält man eine Gesamtkostenkurve C. Erfolgt mit Hilfe staatlicher Interventionen eine Bekämpfung monopolistischer Verhaltensweisen, so gibt sie Auskunft darüber, wie hoch die verbleibenden Effizienzverluste für die Volkswirtschaft bei alternativ angestrebten Monopolisierungsgraden resp. Wettbewerbsintensitäten zu veranschlagen sind. ${ }^{1)}$ Wie Graphik 2 verdeutlicht, ist der optimale Monopolisierungsgrad unter Berücksichtigung der Interventionskosten demzufolge dann realisiert, wenn die Gesamtkosten ihr Minimum erreichen (Punkt $M_{o p t^{*}}$ ). Im Vergleich zu der wirtschaftspolitischén Handlungsanweisung des Konzepts des funktionsfähigen Wettbewerbs, zur Reduzierung der Monopolmacht staatliche Eingriffe bis zum Punkt Mopt auszudehnen, fällt unter Berücksichtigung der Interventionskosten der wirtschaftspolitisch zu empfehlende Interventionsgrad in jedem Fall niedriger aus. In welchem Ausmaß er niedriger ist, hängt sowohl von dem Verlauf der Sozialkostenkurve als auch von dem - je nach Ausgestaltung der statichen Intervention variierenden Verlauf der Interventionskostenkurve ab.

1) Die durch staatliche Interventionen erreichte Senkung der monopolbedingten Sozialkosten kann auch als Ertrag staatlichen Handelns interpretiert werden. Da dieser Ertrag allerdings in Höhe der anfallenden Interventionskosten reduziert wird, ist die Differenz zwischen den sozialen Kosten im extremen Monopolfall und den jeweiligen Gesamtkosten be i alternativen Monopolisierungsgraden als Nettoertrag staatlicher Maßnahmen zu bezeichnen. Dieser Nettoertrag schlägt ab einem bestimmten Interventionsgrad in Nettokosten um, d.h. das Streben nach Abbau der Wettbewerbsbeschränkungen hat dann gesamtwirtschaftliche Effizienzverluste zur Folge, welche die durch Monopolmacht hervorgerufenen Effizienzverluste übersteigen. 
Auch der auf diesem Wege gefundene "optimale" Interventionsgrad kann jedoch für sich U.U. lediglich den Charakter eines Zwischenergebnisses beanspruchen. Bisher wurde die Betrachtung auf die beiden Interventionsebenen "Instrumente" und "Institutionen" beschränkt, d.h. es wurde davon ausgegangen, daß zur Beseitigung eines Marktversagens Interventionen in das Marktpreissystem erfolgen, der Marktpreismechanismus selbst aber nicht zur Disposition steht. Der funktionale Charakter des Wettbewerbs sowie die Suche nach ökonomisch rationalen Entscheidungskriterien erfordern aber geradezu, auch nichtmarktliche Koordinationsmechanismen in die Analyse einzubeziehen. Aus diesem Grunde wäre die oben abgeleitete Empfehlung, zur Eindämmung monopolistischer Verhaltensweisen so lange in das Marktpreissystem einzugreifen, bis die verbleibenden Effizienzverluste (Gesamtkosten) ein Minimum erreichen, dann zu revidieren, falls die Ablösung der Marktpreiskoordination durch eine andere Form gesamtwirtschaftlicher Ressourcensteuerung zu einer weiteren Senkung der Effizienzverluste führt.

In einer ausgeweiteten Analyse ist dann allerdings zu berücksichtigen, daß

1. zwar keine Kosten der Intervention in das Marktpreissystem, wohl aber Interventionskosten im Zusammenhang mit dem Obergang zu einem anderen Koordinationsmechanismus (z.B. Kontingentierung oder Angebot bis zur Sättigung) anfallen;

2. die Transaktionskosten des neuen Koordinationsverfahrens wesentlich höher sein können als die des Marktpreissystems und

3. statt der sozialen Kosten der Monopolisierung nun infolge einer suboptimalen Funktionswe ise des neuen Koordinationssystems ebenfalls beträchtliche soziale Kosten entstehen können. 
Insgesamt läßt sich somit folgendes feststellen: Ob und inwieweit das ökonomische Beurteilungskriterium "monopolistisches Unternehmensverhalten" tatsächlich ein allokatives Marktversagen und damit einen Interventionsbedarf begründet, hängt einerseits von der Höhe der durch die Monopolbildung verursachten sozialen Kosten und andererseits von der Höhe der mit dem Einsatz verschiedener Interventionsformen zur Reduzierung der Wettbewerbsbeschränkungen verbundenen Interventions- und Transaktionskosten $a b$. Die öffentliche Aufgabe ist demnach wie folgt zu formulieren: Die dem Staat zur Verfüguna stehenden Instrumente, die institutionellen Gestaltungsmöglichkeiten und die prinzipiell denkbaren Koordinationsmechanismen sind so zu wählen, daß die durch Monopolisierung bedingte Gesamtkostenbelastuna (Effizienzverluste) für die Volkswirtschaft ihr Minimum erreicht.

Die Anwendung des so gewonnenen Entscheidungskriteriums setzt aufwendige, z.T. mit großen methodischen Schwierigkeiten, aber auch mit erheblichen Meßproblemen verbundene, am Einzelfall zu orientierende empirische Untersuchungen voraus. Daß es daran bisher mangelt, spricht nicht gegen eine solche, im Prinzip richtige Vorgehensweise, sondern nur für die Drinalichkeit einer Intensivierung der Forschungstätigkeit auf diesem Gebiet. 1)

\subsection{Sinkende Durchschnittskosten}

Werden im Regelfall öffentliche Interventionen darauf gerichtet sein, monopolistische Strukturen von vornherein in Grenzen zu halten, zumindest aber die Wettbewerbsbeschränkungen im nachhinein zu korrigieren, so sind darüber hinaus auch Fälle denkbar, in denen die Bereitstellung von Gitern durch konkurrierende Unternehmen zu ineffizienten Ergebnissen führt, es mithin unter allokativen Aspekten wünschenswert ist, wenn als Anbieter

1) Diese Forderung erheben auch $K$. Cowling und D. C. Mueller, a.a.0., S. 746 sowie S. C. Littlechild, a.a.0., S. 360 f. Zu den wenigen empirischen Versuchen, den sozialen Kosten der Monopolisierung den bei ihrer Beseitigung anfallencien sozialen Kosten gegenüberzustellen (allerdings nur im Hinblick auf Regulierungsmaßnahmen) vgl. R. A. Posner, a.a.0., S. $807 \mathrm{ff}$. 
eines bestimmten Gutes nur ein einziges Unternehmen am Markt agiert.

Solche Konstellationen sind ganz allgemein in technologisch ausgereiften Industriegesellschaften, die sich durch einen ständig wachsenden Kapitaleinsatz auszeichen, beobachtbar. Typischerweise treten sie bei der Bereitstellung zahlreicher Infrastrukturleistungen wie z.B. der Energie- und Wasserversorgung, dem Verkehrswesen und der Telekommunikation auf. 1) Die Produktion findet dort unter Bedingungen statt, die durch relativ hohe Fixkosten im Vergleich zu den variablen Kosten gekennzeichnet sind mit der Folge, daB die Kosten je Einheit eines Gutes mit wachsender Ausbringungsmenge sinken. Ob in einem Unternehmen mit sinkenden Durchschnittskosten ${ }^{2}$ gearbeitet, d.h. unter zunehmenden Skalenerträgen produziert wird, hängt jedoch nicht nur vom Anteil der Fixkosten an den gesamten Kosten, sondern ebenso von der Größe des Marktes ab. Häufig erlaubt das Marktvolumen, daB trotz der hohen fixen Kosten mehrere Betriebe gleichzeitig in der Lage sind, eine optimale Auslastung ihrer Kapazitäten zu erzielen. Als weitere Bedingung muß deshalb die Nachfragekurve die Durchschnittskostenkurve im sinkenden $\mathrm{Be}-$ reich schneiden. 3 )

Während in einem Konkurrenzmarkt die gewinnmaximale Produktionsmenge bei Einhaltung der Regel "Grenzkosten gleich Preis" abgesetzt wird, führen - wie die Graphik 3 verdeutlicht - Grenzkostenpreise jedoch zwangsläufig immer dann zu einem Defizit, wenn im relevanten Bereich mit sinkenden Durchschnittskosten gearbeitet wird. Denn unter der Annahme linearer oder ertragsgesetz-

1) Vgl. R. L. Frey, Infrastruktur, a.a.0., S. 76.

2) Von Interesse sind im folgenden stets die langfristigen Durchschnittskosten. Aufgrund der beschränkten Teilbarkeit der Investitionsguiter treten bei Nachfrageschwankungen kurzfristig sinkende Durchschnittskosten häufia als Reflex sich vorübergehend bildender überkapazitäten auf. Dieses Problem ist von dem Phänomen zunehmender Skalenerträge zu trennen; val. dazu auch N. M. Singer, Public Microeconomics, Boston 1972, S. 78 f.

3) Vgl. M. Zumbühl, Privatisierung staatlicher Wirtschaftstätigkeit. Notwendigkeit und Móglichkeiten?, Zürich 1978, S. 91 f. 
licher Kostenverläufe sind sinkende Durchschnittskosten stets gleichbedeutend mit einer oberhalb der Grenzkostenkurve $K^{\prime}$ verlaufenden Durchschnittskostenkurve $K$, was wiederum nichts anderes besagt, als daß die stückkosten höher sind als der zu erzielende Preis. 1) Die im Punkte A realisierte allokationseffiziente Preis-Mengen-Kombination korrespondiert demnach mit einer Ausbringungsmenge $X_{2}$, bei der ein Gesamtverlust in Höhe $\bar{K}_{2} A B P_{2}$ (schraffierte $\left.F l a ̈ c h e ~ 1\right)$ entsteht.

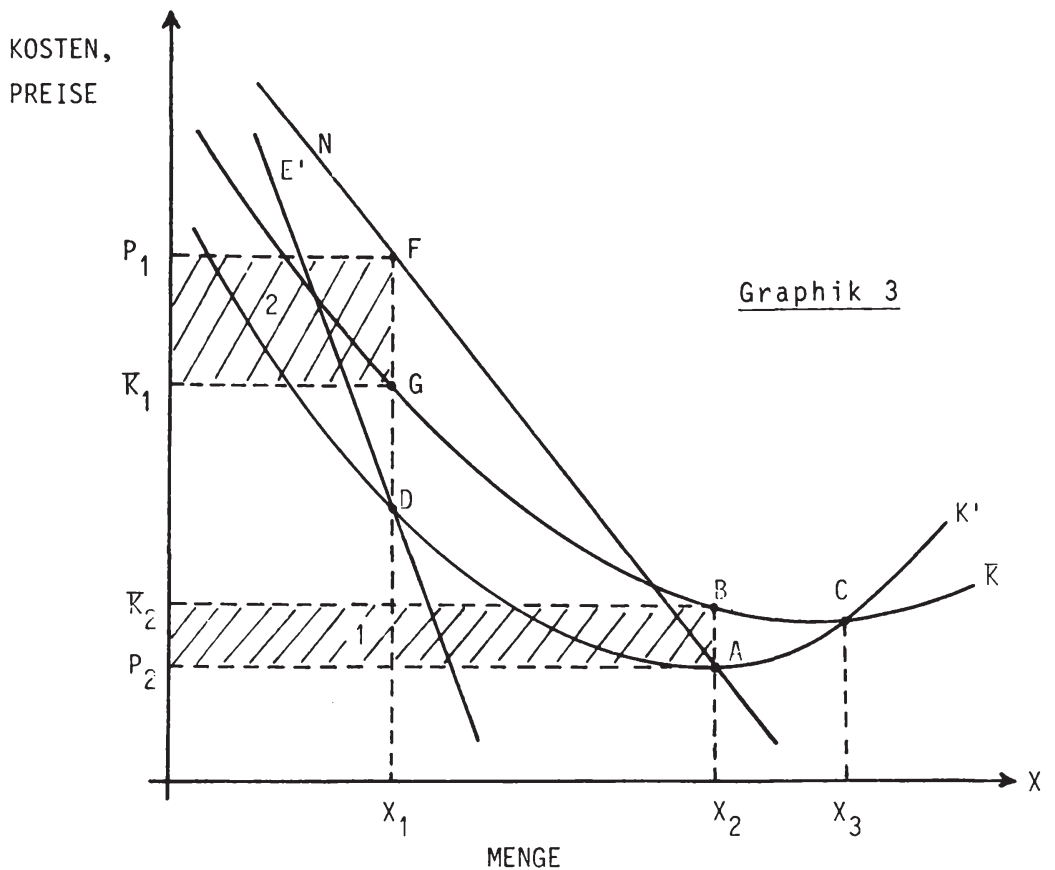

1) Zur analytischen Ableitung siehe dazu L. Johansen, Public Economics, Amsterdam und London 1981, S. $163 \mathrm{ff}$. Vgl. daneben N. M. Singer, a.a.0., S. $77 \mathrm{ff}$. sowie K. Mackscheidt und J. Ste inhausen, Finanzpolitik II. Grundfragen versorgungspolitischer Eingriffe, Tübingen 1977, S. 6 f. 
Bei Aufrechterhaltung der Grenzkosten-Preisregel sind Gewinne erst ab einer Ausbringungsmenge $x_{3}$, also im Schnittpunkt von Grenz- und Durchschnittskosten (Punkt $C$ ), zu realisieren. Um die Vorteile der Kostendegression nutzen zu können, wird ein mit sinkenden Durchschnittskosten arbeitendes Unternehmen deshalb bestrebt sein, seinen Marktanteil gegenüber den Mitbewerbern zu erhöhen. Treten economies of scale dabei in einem Maße auf, daß die Kapazitäten eines einzigen Anbieters bereits ausreichen, die gesamte Marktnachfrage zu bedienen, und zwar zu niedrigeren Kosten, als dies mit einer größeren Zahl konkurrierender Unternehmen möglich wäre, dann ist es wahrscheinlich, daß die Anbieter zeitweilig in einen ruinäsen Wettbewerb eintreten, mit dem Ziel, die gesamte Nachfrage auf sich zu vereinigen. Da aufgrund der produktionstechnischen Bedingungen langfristig nur ein Produzent Oberlebenschancen besitzt, entsteht ein natürliches Monopol.1)

Resultat der Monopolisierung wäre somit eine effizientere Allokation als im Falle der Konkurrenz. Verbleibt nur ein Anbieter am Markt, so wird sein Gewinnmaximierungskalkül allerdings von dem in der Konkurrenzsituation abweichen. Die gewinnmaximale Ausbringungsmenge liegt für den Monopolisten nun bei $x_{1}$, dort wo sich Grenzkosten- und Grenzerlöskurve E' schneiden (Punkt D). Es entsteht ein Monopolgewinn in Höhe von $P_{1} F G K_{1}$, repräsentiert durch die schraffierte Fläche 2 .

Sinkende Durchschnittskosten führen also offenbar zu einer ernsthaften Konfliktsituation zwischen den wirtschaftspolitischen Zielsetzungen "Wettbewerb" einerseits und "effiziente Allokation" andererseits. Unter solchen Bedingungen ist nicht zu erwarten, daß der Marktpreismechanismus ohne weitere Unterstuitzung in der Lage ist, die Koordinationsaufgabe zufrieden-

1) Zum Begriff des natürlichen Monopols vgl. F. Machlup, Art. Monopol, HdSW, Bd. 7, Tübingen 1961, S. 432; vg1. auch N. M. Singer, a.a.0., S. $77 \mathrm{f}$. 
stellend zu lösen. Vielmehr sind - sollen Ineffizienzen vermieden werden - öffentliche Interventionen unumgänglich. 1)

Inwieweit economies of scale einen Interventionsbedarf begründen, hängt nicht allein von der rein theoretisch abgeleiteten bzw. auf bloßen Plausibilitätsüberlegungen beruhenden Feststellung eines Marktversagens ab, sondern ist insbesondere auch eine Frage der empirischen Relevanz sinkender Durchschnittskosten. Eine Reihe vornehmlich in den USA durchgeführter ökonometrischer Studien, die eine überprüfung der Evidenz sinkender Durchschnittskosten für typische Bereiche wie z.B. Elektrizitätsversorgung, Eisenbahn, Wasserversorgung, Muillentsorgung zum Gegenstand haben, ${ }^{2}$ ) konstatieren im Gegensatz zu früheren Untersuchungen ganz überwiegend eine nachlassende Relevanz der economies of scale. 3) Diese empirischen Studien scheinen die Hypothese des Wissenschaftlichen Beirats beim Bundesministerium der Wirtschaft, der Fall sinkender Durchschnittskosten habe "geaenwärtig für öffentliche Unternehmen keine wesentliche Bedeutung" 4 ) zu stützen. Wichtiger noch: Der empirische Befund scheint zu belegen, daß die breite Aufmerksamkeit, die der Produktion mit sinkenden Durchschnittskosten als Kriterium für statliche Interventionen resp. öffentliche Aufgabenerfüllung in der wissenschaftlichen Literatur gewidmet wird, keine Berechtigung besitzt. Bedauerlicherweise stehen jedoch auch solche Hypothesen auf recht unsicheren Fundamenten. Exakte Aussagen über die Verläufe von Kosten- und Nachfragekurven sind nur in seltenen Fällen ohne Schwie-

1) So auch W. S. Vickrey, Decreasing Costs, Publicy Administered Prices, and Economic Efficiency, in: The Analys is and Evaluation of Public Expenditures: The PPB System, A Compendium of Papers submitted to the Subcommittee on Economic in Government of the Joint Economic Committee Congress of the United States, Vol. 1, Washington 1969, S. 121.

2) Einen überblick gibt Ch. B. Blankart, Die wirtschaftspolitische Bedeutung von Skalenerträgen öffentlicher Unternehmen, ZögU 2. Jg. (1979), S. 1 ff.

3) So vor allem L. R. Christensen und W. H. Greene, Economics of Scale in U.S. Electric Power Generation, JPoTEC Vol. 84 (1976), S. 655 ff.; vgl. auch Ch. B. Blankart, a.a.0., S. 15.

4) Wissenschaftlicher Beirat beim Bundesministerium der Wirtschaft, Kosten und Preise offentlicher Unternehmen, Bulletin der Bundesregierung Nr. 4 vom 8.1 .1976$, S. 44 . 
rigkeiten möglich. Überdies bereitet es besondere Probleme, das Phänomen der economies of scale von anderen Tatbeständen zu isolieren, da sinkende Durchschnittskosten häufig nicht offen zutage treten. 1) Die zahlreichen Meßprobleme duirften dann auch der Grund dafür sein, daß es an empirischen Untersuchungen für die Bundesrepublik Deutschland mangelt. Die in den USA durchgeführten Studien mögen für die Bundesrepublik allenfalls gewisse Tendenzaussagen zulassen, eine unreflektierte übertragung der Ergebnisse verbietet sich hingegen.

Welche Formen der statichen Intervention bieten sich an, unterstellt, das Angebot eines bestimmten Gutes erfolge unter den Bedingungen sinkender Durchschnittskosten?

Zunächst ist anzumerken, daB die Feststellung eines Marktversagens infolge der Produktion mit sinkenden Durchschnittskosten noch nicht notwendigerweise bedeutet, daß die Guiterbereitstellung nunmehr über einen anderen Koordinationsmechanismus als den Marktpreis zu erfolgen hat. Eine prinzipiell denkbare Alternative bieten öffentliche Interventionen auf der Instrumentenebene, $d . h$. der Staat schafft die Vorbedingungen für ein Grenzkosten-Preisangebot, indem seine Aktivitäten darauf gerichtet sind, durch Obernahme oder Abbau der Defizite ${ }^{2}$ die Produktion privater Unternehmen zu initiieren. Dabei bieten sich grundsätzlich folgende Möglichkeiten an: ${ }^{3}$ )

1) Vickrey schließt daraus, daß sinkende Durchschnittskosten oftmals unterschätzt werden; vgl. W. S. Vickrey, a.a.0., S. 145.

2) Es scheint der Hinweis angebracht, daß Defizite nicht a priori negativ zu bewerten sind, ist doch in der offentlichkeit die Auffassung weit verbreitet, die Defizite zahlreicher staatlicher und kommunaler Unternehmen seien der schlichte Ausdruck mangelhafter Effizienz des öffentlichen Sektors. Ohne Zweifel sind gerade in diesem Bereich in nicht zu vernachlässigendem Umfang Verluste Z.B. auf versäumte Rationalisierungsmaßnahmen zurückzuführen, von Bedeutung ist jedoch, daß häufig das Prinzip der Kostendeckung gerade deshalb durchbrochen wird, um mittels Setzung optimierender, aber Defizite verursachender Grenzkostenpreise eine Fehlallokation der Ressourcen zu vermeiden.

3) Vgl. M. Zumbüh1, a.a.0., S. $82 \mathrm{f}$. 
- Der Staat trägt die Verluste in voller Höhe;

- Die Verluste werden abgebaut mittels einer Verschiebung der Kostenkurve nach unten, indem der staat die Produktion in einer vorher festgelegten Höhe subventioniert (z.B. in Form von Steuererleichterungen, vergünstigten Darlehen oder Investitionszuschüsen);

- Die Verluste werden abgebaut durch eine Verschiebung der Nachfragekurve nach oben, indem entweder der staat selbst als zusätzlicher Nachfrager fungiert oder aus meritorischen Erwägungen eine Nachfrageerhöhung herbeiführt.

Wie sind diese drei Interventionsformen unter allokativen Gesichtspunkten zu bewerten?

Eine aus dem allgemeinen Steueraufkommen finanzierte staatliche öbernahme der Defizite könnte zwar die Aufrechterhaltung der Grenzkostenpreise bewirken, provoziert aber geradezu unwirtschaftliches Geschäftsgebaren im Unternehmenssektor, denn je höher die verursachten Defizite, umso höher sind auch die den Unternehmen zufließenden öffentlichen Mittel. Ferner ist zu berücksichtigen, daß eine Deckung der Differenz zwischen Durchschnitts- und Grenzkosten durch die Erhebung zusätzlicher Steuern nicht nur - die Allokationseffizienz schmälernde Verwaltungskosten verursacht, sondern daß darüber hinaus negative Wirkungen der Besteuerung auf die belasteten Sektoren die angestrebte Effizienzsteigerung in den begünstigten Sektoren überkompensieren können. 1) überdies scheint es gesamtwirtschaftlich wenig sinnvoll, die Defizite mehrerer Unternehmen abzudecken, wenn nicht auszuschließen ist, daß bereits ein einziger Anbieter ausreicht, die gesamte Nachfrage zu bedienen.

1) Vgl. dazu W. S. Vickrey, a.a.0., S. 124 sowie auch W. J. Baumol und D. F. Bradford, Optimal Departures from Marginal Cost Pricing, AER Vol. 60 (1970), S. $273 \mathrm{ff}$. 
Eine Subventionierung privater Unternehmen ist im Prinzip kaum positiver zu beurteilen als die statliche Defizitdeckung. Zwar kann die Förderung unwirtschaftlichen Unternehmerverhaltens durch die Festlegung der Subventionshöhe eingedämmt werden, gleichwohl ist auch hier mit negativen gesamtwirtschaftlichen Auswirkungen zu rechnen.

Da sinkende Durchschnittskosten nicht für sämtliche Absatzmengen Gïltigkeit besitzen, könnte der öffentliche Sektor als zusätzlicher Nachfrager am Markt auftreten und durch gezielte Auftragsvergabe Unternehmen aus dem Bereich sinkender Durchschnittskosten herausführen. Eine solche Intervention durfte jedoch nur für einige Bereiche (z.B. Bauwirtschaft, Auto- und Rüstungsindustrie) und nur für einen begrenzten Zeitraum in Betracht kommen. Eine durch staatliche Intervention initiierte Nachfrageerhöhung kann darüber hinaus auch aus meritorischen Erwägungen vorgenommen werden. Wird Benutzungszwang ausgeübt, so könnten durch eine Ausweitung der Nachfrage Grenzkostenpreise erzielt werden, d.h. eine effiziente Allokation würde dann quasi per Dekret durchgesetzt. Wird dagegen auf die Ausübung eines Benutzungszwanges verzichtet, so bieten sich die objekt- und die Subjektsubventionierung als alternative finanzielle Anreizinstrumente an. Ohne Kenntnis der speziellen Kosten- und Nachfrageverläufe ist jedoch eine Beurteilung dieser Instrumente unmöglich. U.U. sprechen allerdings ganz andere, grundsätzliche Gesichtspunkte gegen eine Meritorisierung von Gütern und Dienstleistungen. 1 )

Abgesehen von der Tatsache, daß es wegen der begrenzten Möglichkeiten, Telefon-, Wasser-, Gas- und Fernwärmeleitungen mehrfach unter der Erde zu verlegen, bereits technisch unmöglich ist, Wettbewerb aufrecht zu erhalten oder zu erzeugen, verdeuti ichen die Alternativen, daß diese Strategie auch aus ökonomischen Gründen höchst fragwürdig ist. Die Aufrechterhaltung der Marktpreiskoordination erfordert aufgrund der instabilen Wett-

1) Vgl. ausführlich dazu dritter Teil, Abschnitt III, Punkt 2.2. 
bewerbssituation infolge der ständigen Bestrebungen der konkurrierenden Unternehmen, eine Monopolstellung zu erlangen, laufend äußerst aufwendige Eingriffe des Staates zu beträchtiichen Interventionskosten, die insgesamt neue Abweichungen von der effizienten Ressourcenallokation bewirken.

Die unter ökonomischen Gesichtspunkten adäquate Strategie besteht im Falle sinkender Durchschnittskosten demnach offenbar darin, Monopolstellungen zuzulassen und somit das Marktpreissystem weitgehend außer Kraft zu setzen. Gleichzeitig erzeugt die Aufrechterhaltung eines natuirlichen Monopols im Gegensatz zu den sog. küstlichen Monopolen in jedem Falle einen Interventionsbedarf seitens des Staates, da die Möglichkeiten eines natürlichen Monopolisten, seine Stellung in miBbräuchlicher Weise auszunutzen, weit größer sind als die jener Monopole, die aufgrund von Marktunvollkommenheiten entstanden sind. Während bei letzteren das permanente Streben weiterer Unternehmen, auf den Markt zu drängen, den Grad der möglichen Ausbeutung begrenzt, fehlt im Falle sinkender Durchschnittskosten dieser Druck, weil die Produktionskosten zusätzlicher Anbieter stets höher sind als die des Monopolisten, so daß Markteintritte nicht zu erwarten und zudem unter reinen Effizienzgesichtspunkten auch nicht wïnschenswert sind. 1) Daraus ergibt sich zwangsläufig folgende Konsequenz: Die Bereitstellung von unter sinkenden Durchschnittskosten produzierten Gütern über einen anderen Koordinationsmechanismus als den Marktpreis erfordert spezifische und u.U. sehr umfangreiche institutionelle oder/und instrumentelle Interventionen des Staates. M.a.W.: Economies of scale begründen ganz konkrete öffentliche Aufgaben. Vor allem zwei alternative Formen öffentlicher Aufgabenwahrnehmung bieten sich an:

1. Monopolstellungen privater Unternehmen werden der staatlichen Regulierung unterworfen (instrumentelle Intervention).

2. Durch Beteiligung des Staates an privaten Monopolen entstehen gemischtwirtschaftliche Unternehmen, oder durch Ver-

1) Vgl. auch H.S. Vickrey, a.a.0., S. 134. 
staatlichung übernimmt der Staat selbst die Guterbereitstellung, indem er öffentliche Unternehmen errichtet (institutionelle Intervention).

Als staatliche Regulierung können sämtliche hoheitlichen Eingriffe in die individuelle Gewerbe- und Vertragsfreiheit, die nicht lediglich der Festlegung und Durchsetzung alloemein geltender marktwirtschaftlicher Spielregeln dienen, bezeichnet werden. 1) Insbesondere die folgenden vier statlichen Interventionsformen sind nach KAHN unter den Begriff der Regulierung zu fassen: Marktzutrittsbeschränkung, Preiskontrolle, Qualitäts - und Konditionsfestsetzung sowie Kontrahierungszwang. 2 )

Im Gegensatz zu der in den USA bereits recht früh weit verbreiteten staatlichen Regulierung privater Unternehmen (requlated industries) ${ }^{3)}$ spielt in der Bundesrepublik Deutschland und ebenso in den anderen europäischen Staaten traditionell die Intervention auf,institutioneller Ebene, d.h. die teilweise oder völlige Obernahme privater Unternehmen in öffentliches Eigentum, eine wesentlich bedeutsamere Rolle. ${ }^{4}$ ) Dennoch sind auch in der

1) Vgl. J. Muiller und I. Vogelsang, Staatliche Regulierung, Baden-Baden 1979, S. 342. Die staatliche Regulierung ist demzufolge von allaeme inen regulativen Eingriffsformen wie Gebote und Verbote (z.B. generelles Karteliverbot) einerseits und von finanziellen sowie informationellen Instrumenten andererseits abzugrenzen; siehe dazu die Ausführungen im zweiten Teil, Abschnitt II.4. dieser Arbeit. Zu den Teilgebieten der normativen

Theorie der Regulierung vgl. den uberblicksaufsatz von C. C. von Weizsäcker, Staatliche Regulierung - positive und normative Theorie, SZVS 118. Jg. (1982), S. $325 \mathrm{ff}$.

2) Vgl. A. E. Kahn, The Economics of Regulation: Principles and Institutions, Vol. T, New York u.a. 1970, S. 3.

3) Zur Entstehungsgeschichte der staatlichen Regulierung in den USA vgl. B. M. Mitnick, The Political Economy of Regulation. Creating, Designing and Removing Regulatory Forms, New York 1980; vgl. daneben A. E. Kahn, The Economics of Regulation: Principles and Institutions, Vol. 2, New York u.a. 1971 sowie J. Muiller und I. Vogel sang, Staatliche Regulierung, a.a.0., S. $21 \mathrm{ff}$.

4) Zum Vergleich dieser beiden Konzeptionen sowie deren institutionellen Ausgestaltung in den USA und der Bundesrepublik Deutschland siehe insbesondere J. Muiller und I. Vogelsang, Staatliche Regulierung, a.a.0. sowie im überbाick dieselben, Das amerikanische Konzept staatlicher Regulierung im Vergleich mit offentlichen Unternehmen in der Bundesrepublik Deutschland, ZögU 2. Jg. (1979), S. $115 \mathrm{ff}$. 
Bundesrepublik die Elemente einer statichen Regulierung als flankierende Maßnahmen durchaus vorhanden. Dies gilt vor allem für die sog. wettbewerbspolitischen Ausnahmebereiche wie $z$. B. der Elektrizitätsversorgung. ${ }^{1)}$ überwiegend tritt jedoch der Eir satz vorbeugender Regulierungsinstrumente gegenüber einer nachträglich praktizierten Mißbrauchsaufsicht in den Hinterarund.

Ob unter allokativen Zielsetzungen die Regulierung oder die Verstatlichung die effektivere und zugleich effizientere Interventionsform darstellt, ist nicht allgemein guiltig, sondern nur fallweise mit Hilfe auf einzelne Sektoren der Wirtschaft bezogener empirischer Untersuchungen zu beurteilen. Allerdings sind auch hier einige Tendenzaussagen möglich.

Eine Vermeidung des "Nirwana-Ansatzes" setzt die Erkenntnis voraus, daß staatliche Regulierungsaktivitäten unerwijnschte Erscheinungen allenfalls abmildern, niemals jedoch eliminieren können und zudem regulative Interventionen nicht kosten- und friktionsfrei erfolgen. 2) Zunächst fallen direkte administrative Kosten an, die mit der Vornahme konkreter Regulierungsmaßnahmen wie Preiskontrolle, Marktzutrittsbeschränkungen, Festlegung von Qualitätsstandards etc. verbunden sind. Von größerer Bedeutung sind aber im allgemeinen die Kosten, die mit einer Kontrolle der Einhaltung staatlicher Regulierungsmaßnahmen einhergehen. Vor allem diese direkten administrativen Folgekosten der Regulierung führen zu einer Beanspruchung von Ressourcen, die dann für andere Verwendungen nicht mehr zur Verfügung stehen, so daß die mittels Regulierung angestrebte Effizienzsteigerung zu einem - u.U. beträchtlichen - Teil durch die Kosten der Anwendung dieses Instruments geschmälert wird.

1) Neben der in Fußnote 4 der Vorseite genannten Literatur ausführlich dazu $H$. Gröner, Die Ordnung der deutschen Elektrizitätswirtschaft, Baden-Baden 1975; derselbe, Wettbewerbliche Ausnahmebereiche im GWB: Das Beispiel der Elektrizitätsversorgung, in: Handbuch des Wettbewerbs, hrsg. von H. Cox U.a., München 1981, S. $421 \mathrm{ff}$.; U. Brandt, Wettbewerbspol it ische Ausnahmebereiche. Probleme ihrer Identifizierung und Gestaltung, Frankfurt/M. u.a. 1977 sowie G. Bruche, Elektrizitätsversorgung und Staatsfunktion. Das Regulierungssystem der öffentlichen Elektrizitätsversorgung in der Bundesrepublik Deutschland, Frankfurt/M. und New York 1977.

2) So auch J. Miller und I. Vogelsang, Staatliche Regulierung, a.a.0., S. 18 . 
Neben den direkten Interventionskosten fallen darüber hinaus im privaten Sektor indirekte kosten der Regulierung an. So wird beispielsweise die Erfüllung der geforderten Qualitäts standards im Regelfall mit erhöhten Produktionskosten für das regulierte Unternehmen einhergehen. Häufig werden diese indirekten Kosten als weiterer Beleg für Effizienzeinbußen herangezogen, nicht selten avancieren sie sogar zum entscheidenden Argument für die Ineffizienz staatlicher Regulierungsmaßnahmen überhaupt. " ${ }^{1)}$ ubersehen wird dabei, daß die Belastung privater Unternehmen mit zusätzlichen Kosten nur das Resultat einer der mit der Regulierung verfolgten Zielsetzungen ist, nämlich die Internalisierung der durch Monopolstellungen hervorgerufenen negativen externen Effekte. Die Ausnutzung von Monopolstellungen verursacht soziale Kosten, die ebenfalls zu sehr hohen Effizienzverlusten führen können. Den Kosten der Regulierung stehen somit Nutzen gegenüber, die allerdings nur mit großen Schwierigkeiten oder gar nicht zu quantifizieren sind. Dieser Umstand führt regelmäßig zu einer überbetonung der kosten.

Die statiche Regulierung ist also insbesondere auf die Eindämmung bestimmter sozialer Kosten angelegt. Gleichwohl ist kaum zu bestreiten, daß von der Regulierung schließlich auch negative Effekte ausgehen können, die gleichfalls als soziale Kosten - jedoch mit vornehmlich intangiblem Charakter - zu Buche schlagen. Hierzu gehören die mit Regulierungsmaßnahmen unvermeidbar einhergehenden Eingriffe in die Gewerbe- und Vertragsfreiheit privater Unternehmen genauso wie die Einschränkung des Wettbewerbs.

1) So kommt z.B. das Center for the Study of American Business zu dem Eraebnis, daß 1976 die administrativen Kosten der Regulierung ca. 3,2 Mrd. Dollar betrugen, während die dem privaten Sektor auferlegten Kosten mit nahezu 63 Mrd. Dollar weitaus höher ausfielen. "Thus, on the average each dollar that Congress appropriated for regulation resulted in an additional $\$ 20$ of costs imposed on the private sector of the economy." M. L. Weidenbaum, The High Cost of Government Regulation, in: Challenge, Nov. JDec. 1979, S. 37 . 
Die Oberführung privater Unternehmen in öffentliches Eigentum als alternative Interventionsform zur Regulierung scheint zumindest allein keine befriedigende Antwort auf ein Marktversagen infolge sinkender Durchschnittskosten zu bieten, da die Ausgestaltung der Eigentumsverhältnisse für unternehmerische Verhaltensweisen weit weniger prägend ist als dies gemeinhin angenommen wird. 1) Wie zahlreiche Beispiele belegen, neigen öffentliche Monopole nicht weniger als private Monopole dazu, ihre Sonderstellungen mißbräuchlich auszunutzen und damit allokative Friktionen hervorzurufen. Aus dieser Erfahrung ist folgende Konsequenz zu ziehen: Eine überführung von Unternehmen in öffentliches Eigentum entbindet den Gesetzgeber noch nicht von dem wirtschaftspolitischen Erfordernis, flankierende regulative Eingriffe vorzunehmen. 2)

Zumindest die folgenden Argumente sprechen deshalb tendenziell fuir eine Bevorzugung der statichen Regulierung als Interventionsform.

Erstens: Die auch bei Anderung der Eigentumsverhältnisse notwendigen regulativen Eingriffe lassen vermuten, daß die Verstatlichung in höherem Maße Effizienzverluste hervorruft als die staatliche Regulierung unter Beibehaltung privater Monopole.

Zweitens: Die Frage, ob eine öffentliche Aufgabe wegen des Marktversagens infolge sinkender Durchschnittskosten vorliegt oder nicht, bedarf der ständigen überprüfung. Technischer Fortschritt und organisatorische Veränderungen können zu Verschiebungen der Kostenfunktionen und u.U. zu Erweiterungen der Märkte führen mit der Folge, daß ehemals natürliche Monopol-

1) Zur Begründung wird auf die Ausführungen im zweiten Teil, Abschnitt II, Punkt 3.2. verwiesen.

2) Zu den Möglichkeiten und Problemen einer Anwendung des Regulierungsinstrumentariums zur Kontrolle und Effizienzsteigeruna öffentlicher Unternehmen vgl. J. Muiller und I. Vogelsang, Ist eine Effizienzsteigerung der öffentlichen Verwattung durch Anwendung des Instrumentariums der amerikanischen "Public Utility Regulation" möglich?, in: Neuere Entwicklungen in den Wirtschaftswissenschaften, hrsg. von E. Helmstädter, SchdVSocpol N.F., Bd. 98, Berlin 1978, S. $775 \mathrm{ff}$. 
stellungen erodiert werden. " Während sich öffentliches Eigentum im allgemeinen durch eine institutionelle Rigidität ${ }^{2}$ auszeichnet, die verlängerte Reaktionszeiten auf technischen und organisatorischen Wandel erwarten 1 äßt, ist eine Ausgestaltung der Regulierung denkbar, die wesentlich flexibler und wettbewerbsdurchlässiger ist.

Schließlich: In ähnlicher Weise wie bei einer privaten Produktion, die unter der Bedingung sinkender Durchschnittskosten zwangsläufig ein Versagen des Marktpreissystems auslöst, bereitet auch den Entscheidungsträgern des öffentlichen Sektors das Phänomen sinkender Durchschnittskosten Probleme allokativer Art. Der Bau eines modernen Hallenschwimmbades im nahegelegenen Nachbarort einer Gemeinde, die bereits über ein großzügig dimensioniertes Hallenbad verfügt, oder parallele Investitionen in den öffentlichen Nahverkehr und in die Schnellstraßen eines Stadtgebietes sind Beispiele für die Schaffung dubioser Konkurrenzverhältnisse durch öffentliche Planung. Auch hier können sinkende Durchschnittskosten eintreten, die dann das Allokationsziel in ganz beträchtlichem Ausmaße konterkarieren.

1) Als ein Beispiel für die bereits abgeschlossene Erosion einer natürlichen Monopolstellung durch Verschiebung der Nachfrage- und Kostenkurven führt Kaufer den Flugverkehr an; E. Kaufer, Theorie der offentlichen Regulierung, München 1981, S. $156 \mathrm{f}$. Ein jüngeres Beispiel stellt die Telekommunikation dar, die sich gerade in den vergangenen Jahren durch einen raschen technischen Fortschritt auszeichnet, mit der Folge, daß sich auch in diesem Bereich zunehmend Elemente des Wettbewerbs durchsetzen; vgl. G. Knieps, J. Miiller, C. C. von Weizsäcker, Die Rolle des Wettbewerbs im Fernmeldebereich, Baden-Baden 1981.

2) So auch E. Kaufer, a.a.0., S. 173. 


\subsection{Fehlender Ausweis zukünftiger Knappheitsverhältnisse}

Die wirtschaftlichen Aktivitäten eines Unternehmers sind stets mit Risiken behaftet. Das allgemeine Marktrisiko beinhaltet die permanente Unsicherheit, infolge des Konkurrenzdrucks oder aufgrund eigener Fehlplanungen die Wettbewerbsfähigkeit einzubüßen und aus dem Markt ausscheiden zu müssen. Dieses Risiko stellt ein wesentliches und zugleich wünschenswertes Element des marktwirtschaftlichen Systems dar, das keiner Absicherung durch staatliche Interventionen bedarf. Daneben treten kalkulierbare Risiken auf, die durch gewerbsmäßige Versicherungen im Marktprozeß selbst abgedeckt werden können (z.B. Feuer-, Diebstahl-, Produktionsausfallversicherungen). Diesen Risiken sind empirisch beobachtbare Häufigkeiten zugeordnet, so daß sie kalkulierbar werden. Sie können somit als Kostenfaktor in die Preiskalkulation einfließen und haben daher keine negativen Auswirkungen auf die Ressourcenallokation.

Anders sind hingegen jene spezifischen Risiken zu beurteilen, die nicht kalkulierbar und zudem so hoch sind, daß sie, sollten sie eintreten, den Ruin eines Unternehmens zur Folge hätten. Mit diesen hohen, nicht kalkulierbaren Risiken sind insbesondere solche Güter behaftet, die aufwendige und mit völig unsicheren Erwartungen einhergehende Forschungsinvestitionen voraussetzen ( $z . B$. Angebot eines erdölunabhängigen Treibstoffs), aber auch Großprojekte, mit denen unternehmerisches Neuland betreten wird und demzufolge äußerst unsicher ist, ob ein profitables Güterangebot überhaupt bereitgestel1t werden kann (z.B. Kabelkommunikation, Fernwärmeversorgung). Während das Risiko für die Gesamtwirtschaft aufgrund des risikostreuenden Effektes einer großen Zahl solcher Projekte durchaus tragbar ist ${ }^{1}$, wird die Gefahr eines Mißerfolges von einem einzelnen Unternehmen so hoch eingeschätzt, daß ein Angebot vergleichsweise gering aus fällt, wenn nicht gänzlich unterbleibt. Die negativen Auswirkun-

1) $\mathrm{Vgl}$. zur einzel- und gesamtwirtschaftlichen Risikobetrachtung J. Stohler, Zur Methode und Technik der Cost-Benefit-Analyse, in: Kyklos, 20. Ja. (1967), S. $234 \mathrm{f}$. 
gen im Hinblick auf die Allokation der Ressourcen liegen dann auf der Hand. Die Möglichkeiten, mit Hilfe neuer Technologien einen sparsameren Einsatz knapper Mittel zu erreichen, werden nicht voll ausgeschöpft, die Ressourcen mithin fehlgeleitet. Hohe, nicht kalkulierbare Risiken bilden deshalb in der Literatur ein weiteres Kriterium für das Versagen des Marktpreissystems. 1)

Ein aus unzureichender unternehmerischer Risikobereitschaft abgeleitetes Marktversagen bedarf allerdings einer eingehenden Begründung angesichts der Tatsache, daß zweifellos die Initiative privater Unternehmer in ganz entscheidendem Maße mit risikoreichen Innovationen den wirtschaftlichen Entwicklungsprozeß über Jahrhunderte vorangetrieben hat. ${ }^{2}$ )

Eine genauere Analyse der Risikofaktoren gibt bereits AufschluB darüber, daß privatwirtschaftliches Engagement offenbar immer dann unzureichend ausgeprägt ist, wenn

1. der finanzielle Einsatz bezogen auf die Kapitalausstattung eines Unternehmens besonders hoch ist und

2. Gewinne zwar möglich, aber nicht nur äußerst unsicher sind, sondern zudem erst zu einem wesentlich späteren Zeitpunkt anfallen, da die Marktpreise lediglich die gegenwärtigen Be-

1) $\mathrm{Vgl}$. insbesondere $\mathrm{K}$. J. Arrow, Economic Welfare and the Allocation of Ressources for Invention, in: Essays in the Theory of Risk-Bearing, hrsg. von K. J. Arrow, Amsterdam und London 1970, S. $144 \mathrm{ff}$. (erstmals veröffentlicht 1962); R. L. Frey, Infrastruktur, a.a.0., S. 89 f.; K. Mackscheidt und J. Steinhausen, Finanzpol itik II. Grundfragen versorgungspolitischer Eingriffe, a.a.0., S. 7; M. Zumbühl, a.a.0., S. $87 \mathrm{ff}$.

2) "Die Bourgeoisie hat in ihrer kaum hundertjährigen Klassenherrschaft massenhaftere und kolossalere Produktionskräfte geschaffen als andere vergangenen Generationen zusammen. Unterjochung der Naturkräfte, Maschinerie, Anwendung der Chemie auf Industrie und Ackerbau, Dampfschiffahrt, Eisenbahnen, elektrische Telegraphen, Urbarmachung ganzer Weltteile, Schiffbarmachung der Flüsse, ganze aus dem Boden hervorgestampfte Bevölkerungen - welches frühere Jahrhundert ahnte, daß solche Produktionskräfte im Schoße der gesellschaft1 ichen Arbeit schlummerte." K. Marx und F. Engels, Manifest der Kommunistischen Partei, Berlin 1945, S. $8 \mathrm{f}$. 
darfs- und Knappheitsverhältnisse widerspiegeln und deshalb bessere technologische Lösungen vorläufig unterbewerten. 1)

Der zweite Punkt läßt vermuten, daß weniger die vermeintliche Risikoaversion der Unternehmen für eine Fehlallokation der Ressourcen verantwortlich zu machen ist, sondern daß vor allem die Unfähigkeit des Marktpreissystems, die in der Zukunft zu erwartenden Veränderungen der Knappheitsverhältnisse ins Kalkül einzubeziehen, eine effiziente Allokation im Zeitablauf verhindert. Dieses Argument ist am Beispiel des nicht-regenerierbaren Rohstoffs Erdöl leicht zu veranschaulichen. Selbst der weitsichtigste und zugleich risikofreudigste Unternehmer, der die Entwicklung eines vom Erdöl unabhängigen Kraftstoffs vorantreibt und diesen am Markt anbietet, wird u. U. existenzgefährdende Verluste hinnehmen müssen, solange die Benzinproduktion aus Erdöl wesentlich kostengünstiger ist. "Die Logik des Marktes bezieht sich auf die Gegenwart, und sie erlaubt keinen Vorschuß auf die Vernunft, die die Zukunft gebietet."2)

Aus der fehlerhaften Funktionsweise des Marktpreissystems ergibt sich die Notwendigkeit eines öffentlichen Einariffs mit der Direktive, eine auch intertemporal als effizient zu bezeichnende Allokation sicherzustellen. Die öffentliche Aufgabe ließe sich demnach etwa wie folgt formulieren: Neue, ressourcensparende Technologien, deren Entwicklung durch Private infolge eines, die wahren knappheitsverhältnisse verzerrenden, Preissystem unterbleibt, sind entweder - bei Aufrechterhaltung der Marktpreiskoordination - durch den Einsatz staatlicher Instrumente oder Institutionen im privaten Sektor anzureaen oder über nichtmarktliche Koordinationsverfahren einzuführen. Mit der Feststellung eines Interventionsbedarfs bleibt also auch

1) Dieser Risikofaktor wird auch im sechsten Forschungsbericht der Bundesregierung betont; vgl. Bundesminister für Forschung und Technologie (Hrsg.), Bundesbericht Forschung VI, Bonn 1979, S. 28.

2) K. Littmann, Die Chancen staatlicher Innovationslenkung, Göttingen 1975, S. 57, Anm. 1; vgl. auch G. Colm, National Goals Analysis and Marginal Utility Economics, a.a.0., S. 222 . 
hier zunächst die Entscheidung über die zweckmäßige(n) Interventionsebene( $n)$ und -form(en) noch offen. Die übersicht 19 verdeutlicht, daß das Spektrum der denkmöglichen Interventionsalternativen wiederum recht breit ist. Allerdings lassen sich zwei grundsätzliche Ansatzpunkte staatlicher Interventionen unterscheiden:

- Der erste Ansatzpunkt ist vornehmlich darauf gerichtet, die für den Marktpreismechanismus zuweilen nicht untypische Unterbewertung zukünftiger Bedarfs- und Knappheitsverhältnisse zu korrigieren, indem die Nutzungskosten nicht-regenerierbarer Ressourcen erhöht bzw. soziale kosten verursachende Güter und Produktionstechniken diskriminiert werden (z.B. durch Internalisierungssteuern, Formulierung von Standards und Normen).

- Im Blickpunkt des zweiten Ansatzes steht hingegen ein gezieltes Hinwirken auf bestimmte Forschungen und Entwicklungen (selektive Forschungs- und Entwicklungspolitik). 1)

Kann mit der Entscheidung für eine der beiden alternativen Interventionsansatzpunkte bereits eine Vorauswahl unter den Interventionsformen getroffen werden, so sind für eine endguiltige Entscheidung auch in diesem Fall zusätzliche Kriterien notwendig. Zwei - allerdings eng miteinander verknüpfte - oberlegungen dürten dabei allgemeine Relevanz beanspruchen:

Wie bereits in anderen Fällen des Marktversagens beschrieben, sind mit sämtlichen staatlichen Interventionen - also auch mit solchen, die darauf abzielen, eine im Zeitablauf effiziente Allokation zu gewährleisten - Kosten verbunden, welche die Effizienzbemühungen konterkarieren. Gemäß der von DEMSETZ ange-

1) $\mathrm{Vgl}$. K. Littmann, Die Chancen staatlicher Innovationslenkung, a.a.0., S. $78 \mathrm{ff}$. Littmann selbst weist darauf hin, daß die formale Trennung in zwei unterschiedliche Ansatzpunkte des staatlichen Instrumenteneinsatzes erst eine systematische Analyse ermögliche, in der praktischen Politik jedoch Überlappungen der Teilbereiche anzutreffen seien. 
Obersicht 19: Interventionsebenen und -formen bei fehlendem Ausweis zukinftiger Knappheitsverhältnisse

\begin{tabular}{|c|c|}
\hline $\begin{array}{l}\text { INTERVENTIONSEBENE } \\
\text { "INSTRUMENTE" }\end{array}$ & $\begin{array}{l}\text { Informationsbereitstellung: } \\
\text { - Informations- und Dokumentationseinrichtungen (IuD) } \\
\text { - Technologieberatung, Bereitstellung einer "Förder- } \\
\text { fibel" } \\
\text { - Managementhilfen für Innovationen } \\
\text { - Hilfen bei Patentverwertung } \\
\text { Finanzielle Förderung/Bel astung } \\
\text { - Belastung soziale Kosten verursachender Produktions- } \\
\text { techniken (z.B. durch Internalisierungssteuern) } \\
\text { - Steuervergüstigungen } \\
\text { (auch: Sonderabschreibungen, Investitionsprämien } \\
\text { und -zulagen) } \\
\text { - Subventionierung (verlorene Zuschüsse) } \\
\text { - Zinsverbilligte Darlehen } \\
\text { - Bereitstellung von Risikokapital durch finanzielle } \\
\text { Beteiligung (z.B. Wagnisfinanzierungsgesellschaft) } \\
\text { Staatliche Auftragsvergabe } \\
\text { - Spezifizierung von Qualitätseigenschaften der vom } \\
\text { öffentlichen Sektor nachgefragten Güter und Dienste } \\
\text { - Vergabe von Forschungs- und Entwicklungsaufträgen } \\
\text { (Vertragsforschung) } \\
\text { Auflagen, Regulierung } \\
\text { - Formulierung von Emissions- und Immissionsstandards } \\
\text { - Zulassung von Wettbewerbsbeschränkungen in be- } \\
\text { stimmtem Umfang } \\
\text { Ge - und Verbote } \\
\text { - Patentschutz } \\
\text { - Arbeitnehmererfindungsgesetz }\end{array}$ \\
\hline $\begin{array}{l}\text { INTERVENT IONSEBENE } \\
\text { "INST ITUTIONEN" }\end{array}$ & $\begin{array}{l}\text { Gebietskörperschaften } \\
\text { - staatliche Forschunasinstitute } \\
\text { - Staat als "Markterkunder" } \\
\text { Parafiski } \\
\text { Offentliche Unternehmen } \\
\text { Verbände }\end{array}$ \\
\hline $\begin{array}{l}\text { INTERVENT IONSEBENE } \\
\text { "KOORDINATIONS- } \\
\text { MECHANISMEN" }\end{array}$ & $\begin{array}{l}\text { Kontingentierung } \\
\text { Angebot bis zur Sättigung }\end{array}$ \\
\hline
\end{tabular}


regten Vorgehensweise ${ }^{1)}$ ist demnach auch hier darauf zu achten, daß die tatsächlichen Gegebenheiten nicht am Nirwana irgendeines Idealzustandes geprüft, sondern einander reale Alternativen einer intertemporalen Allokation der Ressourcen gegenübergestellt werden. 2)

Die Orientierung an reale Verhältnisse verbietet darüber hinaus die a priori-Unterstellung, öffentliche Handlungsträger seien stets mit einer höheren Risikobereitschaft ausgestattet als private Entscheidungseinheiten. Die Tatsache, daß der Zeithorizont politischer Planungen nicht selten mit dem Ende der Wahlperiode korrespondiert, ist bereits ein Beleg dafür, daß Politiker vor allem geneigt sind, das Risiko der Abwahl zu minimieren. Oberdies zeigen die Erfahrungen, daß Großprojekte, deren Risiken Private als prohibitiv hoch einschätzen, von politischen Akteuren im Regelfall umso eher in Angriff genommen werden, je weniger ein möglicher Fehlschlag sich negativ auf die Wählerstimmen auswirkt, ein Erfolg hingegen in großem Umfang Wählerstimmen sichert ( $z . B$. Mondlandeprojekt). Andererseits scheint im Vergleich zu den am Markt agierenden Unternehmen die Risikobereitschaft öffent1icher Entscheidungsträger immer dann wesentlich geringer ausgeprägt zu sein, sobald Rationalisierungsinnovationen mit der Vernichtung von Arbeitsplätzen einhergehen und sich damit die Chancen der Wiederwahl für den Politiker beträchtlich verringern könnten. 3 )

1) Auch wenn wir die Forderung von Demsetz, das Nirwanadenken in der Okonomie zu überwinden und sich auf die Analyse realer Alternativen zu konzentrieren, allgemein für sämtliche Fälle des Marktversagens übernehmen, so soll an dieser Stelle doch darauf hingewiesen werden, daß der von Demsetz befürwortete Ansatz ursprünglich das Resultat einer kritischen Auseinandersetzung mit einer Arbeit von Arrow über das Marktversagen aufgrund hoher Risiken und dessen Konsequenzen für öffentliche Aktivitäten darstellte; vgl. H. Demsetz, a.a.0., S. $1 \mathrm{ff}$.

2) Das betont auch C. C. von Weizsäcker, Leistet der Markt die optimale intertemporale Allokation der Ressourcen?, in: Erschöpfbare Ressourcen, hrsg. von H. Siebert, SchdVSocpol Bd. 108, Berl in 1980, S. 811.

3) Vgl. dazu H. Demsetz, a.a.0., S. 9. 
Unter Berücksichtigung der Entscheidungskriterien lassen sich einige allgemeine SchluBfolgerungen im Hinblick auf die zweckmäBige Wahl der Interventionsebenen und -formen ziehen:

1. Was die angewandte Forschung und Entwicklung anlangt, so durften im privaten Sektor ausreichende Innovationsreize im Regelfall immer dann induziert werden, wenn der öffentliche Sektor auf der Instrumentenebene unterstuitzend in den Marktmechanismus eingreift, und zwar indem er die Ertragssicherheit der Unternehmen erhöht oder/und die betrieblichen Investitionskosten verringert. ${ }^{1)}$ Dagegen ist die Ablösung des Marktpreissystems durch ein anderes Koordinationsverfahren nicht zu empfehlen, da die in diesem Falle eingesparten Interventionskosten durch vermutiich weit höhere Transaktionskosten überkompensiert wirden.

2. In bezug auf risikoreiche GroBprojekte kann der Stat zwar prinzipiell durch den Einsatz finanzieller Instrumente (z.B. Subventionierung, zinsverbilligte Darlehen) hinreichende Anreize für Private schaffen, jedoch sind selbst dann, wenn keine höhere Risikobereitschaft auf seiten des Staates angenommen wird - temporäre Eingriffe auf der Institutionenebene günstiger zu beurteilen. Betreten nämlich der Staat oder öffentliche Unternehmen als "Markterkunder" unternehmerisches Neuland und zeigt sich, daß ein Güterangebot profitabel bereitgestellt werden kann, so dürte davon eine Signalwirkung auf jene privaten Unternehmen ausgehen, die bis dahin die Risiken infolge mangelnder Vergleichsmöglichkeiten als prohibitiv hoch eingeschätzt haben.

3. Für die Grundlagenforschung ist charakteristisch, daß die erzielten Ergebnisse nicht patentfähig sind, d.h. niemand kann Eigentumsrechte an ihnen geltend machen. Im Hinblick auf die Resultate der Grundlagenforschung versagt also das marktwirtschaftliche AusschluBprinzip. 2) Daraus folgt, daB die Grundlagenforschung vom Staat getragen werden muß, sol-

1) Die in diesem Zusammenhang wesentlichen Eingriffsalternativen auf der Instrumentenebene behandelt M. Zumbühl, a.a.0., S. $88 \mathrm{f}$.

2) Dieser Problemkreis wird unter Punkt 2.1. ausführlich behandelt. 
len Fehlallokationen vermieden werden. Statliche Interventionen sind sowohl auf der Ebene der Institutionen als auch der Koordinationsmechanismen angezeigt. Konkret: Der Staat betreibt Forschungseinrichtungen in eigener Regie bzw. über1 äßt Forschungsaufgaben anderen öffentlichen Trägern (z.B. parafiskalischen Institutionen, öffentlichen Unternehmen oder Verbänden) und gewährleistet, daß die Resultate allgemein zugänglich werden.

\subsection{Externe Effekte der Produktion}

Ein "externer Effekt" entsteht immer dann, wenn die Produktion bzw. der Konsum eines Wirtschaftssubjektes positive oder negative Wirkungen auf die Produktions- oder Konsumbedingungen eines anderen Wirtschaftssubjektes ausübt, ohne da $B$ der Verursacher negativer Wirkungen belastet (bei positiven Wirkungen entschädigt) und der Betroffene entschädigt (bei positiven Wirkungen belastet) wird. Anders formuliert: In die Produktionsfunktion eines Unternehmens bzw. in die Nutzenfunktion eines Haushalts fließen Größen ein, die nicht lediglich von seinen eigenen Aktivitäten, sondern auch von den Handlungen anderer Wirtschaftssubjekte abhängen. ${ }^{1)}$

Obersicht 20 bietet anhand von Beispielen eine Zusammenstellung der unterscheidbaren Kategorien externer Effekte. ${ }^{2)}$ Es handelt sich dabei ausschließlich um "technologisch" bedingte Externalitäten, die üblicherweise streng von den sog. "pekuniären" externen Effekten getrennt werden. Letztere entstehen aufgrund von Anderungen der relativen Preise der Güter und Produktionsfaktoren. Erhöht sich z.B. in einer expandierenden Wirtschafts-

1) Zur formalen Definition externer Effekte vgl. J. M. Buchanan und W. C. Stubblebine, Externality, in: Economica, N.S., VoT. 29 (1962), S. 372 sowie E. J. Mishan, The Postwar Literature on Externalities: An Interpretative Essay, JEL Bd. 9 (1971), S. 2 f.

2) Externe Effekte wirken jedoch nicht ausnahmslos in eine Richtung, sondern können auch gegen- oder mehrseitige Beziehungen aufweisen; vgl. dazu das anschauliche und deshalb oft zitierte Beispiel des Imkers und des Obstbauern bei J. C. Meade, External Economies and Diseconomies in a Competitive Situation, in: Economic Journal, Vol. 62 (1952), S. 56 ff. 
Obersicht 20: Kategorien externer Effekte

\begin{tabular}{|c|c|c|c|}
\hline $\begin{array}{r}\text { Entstehung } \\
\text { bereic }\end{array}$ & $\begin{array}{r}\text { Wirkungs- } \\
\text { bereich }\end{array}$ & PRODUKTION & KONSUM \\
\hline & $\begin{array}{l}\text { externe } \\
\text { Nachteile }\end{array}$ & $\begin{array}{l}\text { Flußverunreinigung } \\
\text { durch Abwässer eines } \\
\text { Chemiewerkes } \longrightarrow \\
\text { Verringerung der Fang- } \\
\text { ergebnisse für ansässi- } \\
\text { ges Fischereigewerbe }\end{array}$ & $\begin{array}{l}\text { Flußverunreinigung durch } \\
\text { Abwässer eines Chemie- } \\
\text { werkes } \longrightarrow \\
\text { Das Baden im FluB wird } \\
\text { für Anwohner unmöglich }\end{array}$ \\
\hline & $\begin{array}{l}\text { externe } \\
\text { Vorteile }\end{array}$ & $\begin{array}{l}\text { Bau eines Staudamms } \\
\text { durch ein Elektrizitäts- } \\
\text { werk } \longrightarrow \\
\text { Ackerland der Bauern } \\
\text { wird vor Oberflutung } \\
\text { geschitzt }\end{array}$ & $\begin{array}{l}\text { Bau eines Staudamms } \\
\text { durch ein Elektrizitäts- } \\
\text { werk } \longrightarrow \\
\text { Schaffung eines Ausflugs- } \\
\text { ziels für Touristen }\end{array}$ \\
\hline \multirow{2}{*}{ KONSUM } & $\begin{array}{l}\text { externe } \\
\text { Nachteile }\end{array}$ & $\begin{array}{l}\text { Zerstörung von Weide- } \\
\text { land durch Skifahrer } \longrightarrow \\
\text { Verringerung der Erträge } \\
\text { aus Viehwirtschaft für } \\
\text { ansässige Bergbauern }\end{array}$ & $\begin{array}{l}\text { Qualm der Raucher } \\
\text { im Restaurant } \longrightarrow \\
\text { Einschränkung des Genusses } \\
\text { für nichtrauchende } \\
\text { Gäste }\end{array}$ \\
\hline & $\begin{array}{l}\text { externe } \\
\text { Vorteile }\end{array}$ & $\begin{array}{l}\text { Private Weiterbildung } \\
\text { eines Arbeitnehmers } \longrightarrow \\
\text { Erhöhung der Leistungs- } \\
\text { kraft des Unternehmens }\end{array}$ & $\begin{array}{l}\text { Blumenbeet eines } \\
\text { Gartenbesitzers } \longrightarrow \\
\text { Augenweide für Spazier- } \\
\text { gänger }\end{array}$ \\
\hline
\end{tabular}

branche die Nachfrage nach einem knappen Produktionsfaktor, so hat eine allgemeine Verteuerung dieses Produktionsmittels auch Einflusse auf andere Wirtschaftszweige, die diesen Produktionsfaktor verwenden. Solche Interdependenzen gehören indes zu den konstitutiven und zugleich wünschenswerten Eigenschaften des 
Marktpreissystems, d.h. sie sind für das eigentliche ökonomische Problem der externen Effekte völlig irrelevant. ${ }^{1)}$ Die folgenden Ausfühungen beschränken sich daher naturgemäB auf technologische externe Effekte. Ferner sind - da hier die Angebotsseite des Wirtschaftsprozesses betrachtet wird - nur die externen Effekte der Produktion von Interesse. ${ }^{2)}$ AuBerdem werden aus analytischen Gründen die nachfolgenden Oberlegungen auf externe Nachteile begrenzt, obschon externe Vorteile und Nachteile in gleichem Maße eine fehlerhafte Marktpreissteuerung der Ressourcen bewirken. Schließlich erfolgt die Darstellung der externen Nachteile exemplarisch anhand des Umweltbereiches. Freilich lassen sich die Argumente unschwer auf andere Bereiche übertraden.

Welche allokativen Konsequenzen sich aus der Existenz externer Nachteile ergeben, läBt sich anhand eines Beispiels illustrieren. Die beiden konkurrierenden Chemiewerke $A$ und $B$ haben über eine möglichst kostengünstige Beseitigung ihrer Produktionsabfälle zu befinden. Das Werk A entschlieBt sich, seine Abfälle in einen nahegelegenen $F l u B$ einzuleiten und somit unentgeltich die Kapazitäten eines offenbar freien Gutes zu nutzen. Das Werk $B$, das keinen Zugang zum FluB hat, muB dagegen seine Abfälle abtransportieren und dafür beträchtliche Kosten veranschlagen. Das Werk $A$ besitzt mithin einen Kostenvorteil gegenüber dem Werk B, der es A ermöglicht, zu niedrigeren Preisen anzubieten. Werden daraufhin die Produkte des A stärker nachgefragt, so geht damit eine zunehmende FluBverschmutzung einher mit der Folge, daB andere Wirtschaftssubjekte die Kapazitäten des Flusses nur noch in wesentlich geringerem Umfang oder überhaupt nicht mehr nutzen können (z.B. Fischereigewerbe, Erholungssuchende, Schwimmer). Tatsächlich nimmt das Chemiewerk A also kein

1) Mishan hat diese auf Viner (1931) zurückgehende und in der Literatur seltsamerweise weit verbreitete Unterscheidung zwischen pekuniären und technologischen externen Effekten zu Recht als einen völliq überfluissigen Beitrag zur Verwirrung kritisiert; vgl. E. J. Mishan, The Postwar Literature on Externalities, a.a.0., S. 6. Gerade deshatb aber ist es bedeutsam, auf diesen Sachverhalt nochmals hinzuweisen.

2) Die externen Effekte des Konsums werden im Zusammenhang mit dem Problemkreis der öffentlichen Güter behandelt. 
freies Gut, sondern - ohne dafür finanziell belastet zu werden die knappe Ressource Umwelt in Anspruch, die für alternative Verwendungen dann nur noch eingeschränkt oder gar nicht mehr zur Verfügung steht. Die Dritten auferlegten Belastungen gehen folglich auch nicht als Kosten der Umweltnutzung in die Produktpreise des Chemiewerks ein, sondern werden als gesamtwirtschaftliche Kosten externalisiert.

Externe Nachteile bewirken daher ein Auseinanderfallen der privatwirtschaftlichen und der gesamtwirtschaftlichen Produktionskosten mit dem Resultat, daß die Marktpreise die tatsächlichen Knappheitsverhältnisse der Produktionsfaktoren und Güter falsch wiedergeben. Die Steuerung der Ressourcen über den Marktpreis mechanismus verhindert demzufolge das Zustandekommen einer effizienten Allokation.

Ist aber mit der Identifikation externer Nachteile ein Bedarf an staatlichen Interventionen in jedem Fall hinreichend begründet?

Diese Frage kann ohne weiteres verneint werden, denn das tägliche Leben weist eine solche Vielzahl von Externalitäten auf, daB - würde man das Argument generell zur Rechtfertigung staatlicher Eingriffe heranziehen - eine Allzuständigkeit des öffentlichen Sektors die unumgängliche Konsequenz wäre. Es muß deshalb wohl pragmatischer vorgegangen und zwischen harmlosen und gravierenden Fällen externer Effekte unterschieden werden. M.a.W.: Erst die Oberschreitung einer konkret zu definierenden Relevanzschwelle ist konstitutiv für einen Interventionsbedarf. 1) Ein solches Vorgehen besitzt zwar den unbestreitbaren Vorteil der Praxisnähe, ist jedoch andererseits mit dem Nachteil behaftet, daB externe Effekte nun nicht mehr eindeutig mit Hilfe ökonomischer Kategorien festzulegen sind. Selbst die naturwissenschaftlich-medizinische Analyse im Bereich des Umweltschutzes ist offenbar nicht in der Lage, objektive Relevanzschwellen exakt zu

1) Auch Musgrave stellt fest, daß "... budgetary provision is usually reserved for cases where externalities weigh quite heavily in the total picture." R. A. Musgrave, Provision for Social Goods in the Market System, a.a.0., S. 311 
bestimmen, wie die abweichenden Toleranzgrenzen im Ländervergleich dokumentieren. ${ }^{1)}$ Unter Umständen ist allerdings die mangelnde Obereinstimmung der Schädigungsgrenzen nicht ausschließlich auf einen unzureichenden oder unterschiedlichen Stand der naturwissenschaftlich-medizinischen kenntnisse zurückzuführen, sondern sie ist ebenso auch AusfluB divergierender gesellschaftlicher und politischer Umfelder. ${ }^{2)}$

Begrüden aber relevante externe Effekte stets statiche Interventionen?

Auch in diesem Fall ist eine differenzierte Antwort angebracht. Nach Auffassung einiger Autoren kann auch bei relevanten externen Nachteilen der Marktmechanismus u.U. selbst in der Lage sein, eine effiziente Allokation herbeizuführen. Statliches Handeln wäre dann lediglich zur Modifikation ordnungspolitischer, insbesondere eigentumsrechtlicher Rahmenbedingungen erforderlich. Als Internalisierungsverfahren werden in diesem Zusammenhang die Fusion und Verhandlungen genannt.

Mit der Fusion von Unternehmen wird das Ziel verfolgt, mittels Zusammenschluß der Verursacher und Betroffenen die externen Effekte gegenseitig aufzurechnen und damit zu internalisieren. Dieses Verfahren zur Lösung des Externalitätenproblems erscheint auf den ersten Blick bestechend, verliert bei genauerer Analyse jedoch schnell an Attraktivität. Es treten vor allem folgende Probleme auf: ${ }^{3)}$

- Eine Internalisierung ist ohne Schwierigkeiten nur möglich, wenn die beteiligten Unternehmen das gleiche Produkt herstellen. Solche Konstellationen dürten aber die Ausnahme darstellen, üblicherweise gehören Verursacher und Betroffene verschiedenen Wirtschaftszweigen an (z.B. Chemiewerk und

1) Vgl. R. Kösters, Die Regulierung externer Effekte, Tübingen 1979, S. $17 \mathrm{f}$.

2) Bereits die Festlegung von Relevanzschwellen im Hinblick auf externe Effekte wird damit zum Gegenstand des politischen Prozesses; vgl. dazu R. Kösters, a.a.0., S. $18 \mathrm{f}$. sowie ausführlich S. $116 \mathrm{ff}$.

3) Vgl. dazu auch K. Littmann, Umweltbelastung - Sozialökonomische Gegenkonzepte, Göttingen 1974, S. $56 \mathrm{f}$. 
Fischerei, Papiermühle und Wäschereibetrieb). Inwieweit eine Verbesserung der Ressourcenallokation in diesen Fällen zu erreichen ist, hängt dann wesentlich von den Möglichkeiten des betrieblichen Rechnungswesens ab, einen Ausgleich zwischen den Kostenstellen herbeizuführen.

- In der Regel haben die externen Effekte der Produktion nicht nur Auswirkungen im Produktionssektor selbst, sondern beeinflussen gleichzeitig auch den Konsum. Deshalb werden mittels Fusion günstigenfalls ein Teil der externen Effekte internalisiert. Im Konsumbereich kann dann aber u.U. eine Verstärkung der externen Nachteile eintreten, falls nämlich die Kostendegression als Resultat der Fusionierung einen höheren Produktionsoutput ermöglicht, der wiederum die Umweltbelastungen verstärkt.

- Schließlich ist nicht auszuschließen, daB mit der Fusion von Unternehmen unerwünschte Wettbewerbsbeschränkungen einhergehen, die den positiven Allokationseffekt zumindest schmälern, eventuell aber auch völlig zunichte machen oder sogar ins Negative verkehren.

Die Fusion als eine "marktgerechte" Internalisierungsvariante düfte deshalb wohl nur in wenigen Ausnahmefällen zur Lösung des Externalitätenproblems beitragen.

Nach COASE ${ }^{1)}$ ist die Abweichung der einzelwirtschaftlichen von den gesamtwirtschaftlichen Kosten ausschließlich durch die unzureichende Fixierung der individuellen Eigentumsrechte (z.B. der Nutzungsrechte für die Umwelt) bedingt. Gelingt es, Rechtspositionen eindeutig zu markieren, so führen Verhandlungen zwischen Verursacher und Betroffenem zur Internalisierung der externen Effekte, und zwar - dies ist besonders bedeutsam - unabhängig davon, ob die konkrete staatliche Recht-

1) Vgl. R. Coase, The Problem of Social Cost, a.a.0., S. $1 \mathrm{ff}$. 
setzung dem Verursacher die Verbreituna externer Nachteile gestattet oder untersagt bzw. der Betroffene ein Recht auf Unterlassung besitzt oder nicht. 1 )

Ist etwa - um das Beispiel wieder aufzunehmen - dem Chemiewerk die Einleitung von Abwasser gestattet, so besteht seitens der geschädigten Fischer ein Anreiz, mittels Ausgleichszahlungen das Chemiewerk zu einem teilweisen oder völligen Verzicht auf dessen Nutzungsrechte zu bewegen. Haben umgekehrt die Fischer ein Recht auf Nichtbeeinträchtigung, so wäre es für das Chemiewerk von Vorteil, diese für die Duldung der externen Nachteile zu entschädigen. Anders formuliert: Indem entweder die Fischer das Recht des Chemiewerks auf ungehinderte Ausübung wirtschaftlicher Betätigung ${ }^{2)}$ gegen Entgelt erwerben oder das Chemiewerk den Fischern jhr Recht auf Nichtbeeinträchtigung abkauft, erfolgt auf dem Verhandlungswege eine Internalisierung der externen Effekte, die - im Idealfall - für alle Beteiligten Verhandlungsgewinne verspricht.

Der besondere Reiz der von COASE propagierten und seit einigen Jahren wieder lebhaft diskutierten Verhandlungslösung ${ }^{3)}$ liegt vor allem in der Aussicht, Externalitäten "marktfähig" zu machen, d.h. die Spielregeln des Marktpreissystems z.B. auch auf das Handeln mit individuellen Rechten der Umweltnutzung auszudehnen, so daß staatliche Interventionen zur Lösung des Externalitätenproblems weitgehend überflüsig werden.

1) Coase wendet sich damit gegen die bis dahin vorherrschende Ansicht, daB ausschlieBlich der Verursacher negativer externer Effekte belastet werden sollte (Pigou-Steuer). Für inn handelt es sich vielmehr um ein reziprokes Problem, d.h. Verursacher und Betroffener sind gleichermaßen in Betracht zu ziehen. Die Kardinalfrage lautet dann: "Should A be allowed to harm B or should B allowed to harm A?" R. Coase, The Problem of Social Cost, a.a.0., S. 2 .

2) Exakt formuliert handelt es sich bei dieser Variante um ein System ohne jegl iche Schadenshaftung.

3) Zur Diskussion im deutschen Schrifttum vgl. K. Jaeger, Steuern, Entschädigungen und das Coase Theorem, in: Jahrbuch für Sozialwissenschaft Bd. 26 (1975), S. $211 \mathrm{ff}$. und die sich daran anschließende Auseinandersetzung mit Endres; vgl. auch $A$. Endres, Die Coase-Kontroverse, $\mathrm{ZfgS}$ Bd. 133 (1977), S. 637 ff. sowie L. Wegehenkel, Coase-Theorem und Marktsystem, Tübingen 1980. 
Ahnlich wie die Fusion besitzen allerdings auch Verhandlungen zwischen Verursachern und Betroffenen bisher nur recht bescheidene Bedeutung für die Wirtschaftspolitik. Dies dürte vor allem darin begründet liegen, daB COASE seine Verhandlungslösung unter äuBerst restriktiven Annahmen, insbesondere unter Vernachlässigung jeglicher Transaktionskosten ${ }^{1)}$ formuliert hat. ${ }^{2}$ ) Die in der Realität anfallenden Transaktionskosten lassen die Existenz externer Effekte jedoch überhaupt erst zu einem Problem werden, und sie erweisen sich zugleich als eine recht hohe Hürde für die Verwirklichung der Verhandlungslösung. Sind z.B. die Geschädigten bereit, ihre Rechte den Verursachern gegen Entgelt abzutreten, so müssen sie nicht nur ihre externen Nachteile genau ermitteln (Schadensermittlungskosten), sondern auch die Vorteile kennen, die die Verursacher aus den Verhandlungen ziehen (Vorteilsermittlungskosten). Die Betroffenen müssen zudem die Verursacher ausfindig machen (Identifikationskosten) und ihnen ihre Bereitschaft zu Verhandlungen signalisieren (Informationskosten). Sie haben darüber hinaus innerhalb der eigenen Gruppe der Betroffenen Einigkeit zu erzielen (interne Konsensfindungskosten) und im eigentlichen VerhandlungsprozeB die Bedingungen mit den Verursachern auszuhandeln (externe Konsensfindungskosten). SchlieBlich müssen die Betroffenen darauf achten, daß die Schädiger die mit ihnen geschlossenen Verträge einhalten (Kontrollkosten). ${ }^{3)}$ Analog fallen auch bei den Verursachern Transaktionskosten an. Die Verhandlungen werden dann scheitern, wenn die Transaktionskosten die potentiellen Verhand-

1) Allerdings weist er selbst auf die Notwendigkeit hin, Transaktionskosten zu beruicksichtigen; vgl. R. Coase, The Problem of Social Cost, a.a.0., S. $15 \mathrm{ff}$.

2) Als weitere Prämisse wird vollständige Konkurrenz auf allen Märkten unterstellt; ebenda, S. 2.

3) Zur Bedeutung der Transaktionskosten vgl. auch D. Cansier, Marktversagen und staatliche Guterbereitstellung als Merkmale öffentTicher Guiter, in: Public Finance, Bd. 27 (1972), S. 432 f. Verschiedene Typologien der Transaktionskosten finden sich auch be $\mathrm{C}$. J. Dahlmann, The Problem of Externality, a.a.0., S. $143 \mathrm{ff}$. Sowie L. Wegehenkel, Coase-Theorem und Marktpreissystem, a.a.0., S. $6 \mathrm{ff}$. 
lungsgewinne übertreffen. Da die Transaktionskosten mit zunehmender Zahl der Verhandelnden - vermutlich überproportional - steigen, nimmt tendenziell mit zunehmender GruppengröBe die Wahrscheinlichkeit einer Verhandlungslösung ab.

Grundsätzlich ist es denkbar, daB der Staat durch eine exakte und situationsgerechte Ausgestaltung der Rechtspositionen Spielräume für die Senkung der Transaktionskosten schafft ${ }^{1}$ ) und damit das Zustandekommen einer Verhandlungslösung günstig beeinfluBt, z.B. indem er den Grad der Betroffenheit durch Verschmutzung und Lärmbelästigung in einem Industriegebiet anders definiert als in einem reinen Wohngebiet. Gleichwohl dürten Verhandlungen über Externalitäten wohl allenfalls in kleinen Gruppen zustandekommen und selbst dann sind allokative Verbesserungen ungewiß, da mit zusätzlichen Hemmnissen zu rechnen ist: ${ }^{2}$ )

- Der Verursacher muß die Rechte sämtlicher Geschädigten erwerben. Bereits eine einzige Verweigerung führt zum Scheitern der Verhandlungen.

- Die Verhandlungslösung wird verhindert, falls der Verursacher derart hohe Zugeständnisse machen muB, daß seine finanziellen Möglichkeiten zur Befriedigung aller Ansprüche gesprengt werden.

- Läuft die Produktion bereits, so ist nicht auszuschließen, daB die Geschädigten ihre wahren Präferenzen verberaen und statt dessen die maximale Zahlungsbereitschaft des Verursachers ausloten (Ausbeutung).

Wird akzeptiert, daB der marktwirtschaftliche Königsweg, die Internalisierung externer Nachteile mittels Fusion oder Verhandlung, nur in Ausnahmefällen in Richtung allokativer Verbesserungen führt, so bedeutet dies, daB mit der Identifika-

1) In der Literatur wird häufig übersehen, daß Coase selbst auf diese Möqlichkeit aufmerksam macht; vgl. R. Coase, The Problem of Social Cost, a.a.0., S. 16 .

2) Vgl. dazu K. Littmann, Umweltbelastung - Sozialökonomische Geqenkonzepte, a.a.0., S. $58 \mathrm{ff}$. 
tion relevanter externer Effekte in der Regel staatliche Interventionen notwendig werden. Mit dieser wirtschaftspolitischen Grundsatzentscheidung im Hinblick auf die Behandlung externer Nachteile sind unmittelbare Konsequenzen auch für das Gut Umwelt selbst verbunden. Reine Luft, sauberes Wasser, Ruhe etc. sind nun nicht mehr als primär private Güter zu betrachten, die von den Wirtschaftssubjekten über den Markt angeboten und nachgefragt werden können, sondern der Staat weist den privaten Akteuren individuelle Rechte der Umweltnutzung über nichtmarktliche Koordinationsmechanismen zu. Damit erhält die Umweltqualität einen iberwiegend öffentlichen Charakter. Weiter: Mit der Wahl einer bestimmten Interventionsform zur Reduzierung der externen Nachteile wird simultan auch eine Entscheidung über das konkrete Koordinationsverfahren zur Bereitstellung des Gutes Umwelt getroffen. So haben z.B. Produktnormen oder Auflagen bezüg1 ich der Produktionstechnik eine Kontingentierung des Gutes Umwelt zur Folge, während direkte staatliche Umweltschutzinvestitionen gleichbedeutend sind mit einem Angebot des Gutes Umwelt bis zur Sättigung. Die Interdependenzen zwischen den Interventionsformen zur Reduzierung der Umweltschäden einerseits und der Art und Weise der Bereitstellung des Gutes Umwelt andererseits sind stets zu beachten.

In Obersicht 21 sind die wichtigsten grundsätzlich anwendbaren Eingriffsmöglichkeiten dargestellt. Konkrete Hinweise darauf, welche Interventionsalternativen sich für die Lösung des Externalitätenproblems als geeignet erweisen, insbesondere zur Beantwortung der Grundsatzfrage, ob Interventionen in den Marktpreismechanismus oder solche zur Ablösung der Marktpreiskoordination das geeignete Mittel sind, lassen sich zwar wiederum nur mit $\mathrm{Hilfe}$ von Detailuntersuchungen geben, dennoch können auch hier einige Wegmarkierungen angebracht werden.

Vorweg: Bei allen Einwänden gegen das Coase-Theorem sollte nicht übersehen werden, daB COASE mit seinen Oberlegungen den Blick geschärft hat für die Notwendigkeit der Analyse realistischer Alternativen wirtschaftspolitischen Handelns. Denn nicht 
Obersicht 21: Interventionsebenen und -formen bei

externen Effekten der Produktion

\begin{tabular}{|c|c|}
\hline $\begin{array}{l}\text { INTERVENTIONSEBENE } \\
\text { "INSTRUMENTE" }\end{array}$ & $\begin{array}{l}\text { Informationsbereitstellung: } \\
\text { - Berichte (z.B. Umweltberichte der Bundesregierung) } \\
\text { - Beratung (z.B. uber Möglichkeiten des Recyclings) } \\
\text { - Appelle (z.B. Energiesparappelle) } \\
\text { - positive und negative ideelle Anreize (z.B. öffent- } \\
\text { liche Belobigung für vorbildliches Umweltverhalten } \\
\text { bzw. öffentliches Anprangern umweltbelastender } \\
\text { Aktivitäten) } \\
\text { Finanzielle Förderung/Belastung: } \\
\text { - Subventionen (Subventionierung umweltfreundlicher } \\
\text { Produkte, Subventionierung der Schadstoffbeseiti- } \\
\text { gung; Abschreibungserleichterungen u.a.) } \\
\text { - Steuern (Emissionssteuer, Inputsteuer, Produkt- } \\
\text { steuer etc.) } \\
\text { - Gebühren } \\
\text { Auftragsvergabe: } \\
\text { - Staatliche Nachfrage nach umweltfreundlichen Gütern } \\
\text { (z.B. Kfz, die mit bleifreiem Benzin betrieben } \\
\text { werden) } \\
\text { Auflagen, Regulierung, Ge- und Verbote: } \\
\text { - Emissionsstandards } \\
\text { - Normen über anzuwendende Produktionsverfahren } \\
\text { - Produktnormen } \\
\text { - Produktmengenlimitierung } \\
\text { - Verbot bestimmter Produkte oder Produktionstechniken } \\
\text { - Ansiedlungsverbote }\end{array}$ \\
\hline $\begin{array}{l}\text { INTERVENTIONSEBENE } \\
\text { "INST ITUTIONEN" }\end{array}$ & $\begin{array}{l}\text { Gebietskörperschaften: } \\
\text { - direkte Umweltschutzmaßnahmen durch öffentliche } \\
\text { Investitionen (z.B. Kläranlagen) } \\
\text { offentliche Unternehmen: } \\
\text { - Bereitstellung konkurrenzfähiger, umweltfreundlicher } \\
\text { Güter (zusätzlich notwendiges Instrument: staat- } \\
\text { liche Subventionen) } \\
\text { Zusammenschluß Privater: } \\
\text { - Proteste und Appelle von Bürgerinitiativen, die so- } \\
\text { ziale Sanktionen gegen Umweltsünder bewirken }\end{array}$ \\
\hline $\begin{array}{l}\text { INTERVENTIONSEBENE } \\
\text { "KOORDINATIONS- } \\
\text { MECHANISMEN" }\end{array}$ & $\begin{array}{l}\text { Kontingentierung: } \\
\text { - Bereitstellung der mit externen Nachteilen behafte- } \\
\text { ten Güter nach persönlichem oder sachlichem Merkmal } \\
\text { Angebot bis zur Sättigung: } \\
\text { - Bereitstellung umweltfreundlicher Substitutions- } \\
\text { guiter zu niedrigen "Preisen". (Tarifen) } \\
\text { AlbertPeters - } 978-3-631-75163-3\end{array}$ \\
\hline
\end{tabular}


allein die externen Effekte verursachen Fehlallokationen und damit soziale Kosten für die Volkswirtschaft, sondern ebenso gehen mit Interventionen zur Internalisierung der Externalitäten Kosten einher, die gleichbedeutend sind mit einem Verzehr knapper Ressourcen. Zudem muB auch hier stets mit einer lediglich imperfekten Korrektur der Funktionsstörungen des Marktpreissystems durch Eingriffe des öffentlichen Sektors gerechnet werden. Mit der Suche nach braüchbaren Lösungen überwindet COASE damit bereits den später von DEMSETZ beklagten Nirwana-Ansatz der Wohlfahrtsökonomik und fordert - wie später DEMSETZ - die Berücksichtigung des Gesamteffekts alternativer wirtschaftspolitischer Entscheidungen. ${ }^{1)}$

Sind die Marktpreise nicht in der Lage, die wahren Knappheitsverhältnisse der mit externen Effekten behafteten Guiter anzuzeigen, so ist zu prüfen, ob in diesen fällen der Obergang von der Marktpreiskoordination zu einem anderen Verfahren der Güterbereitstellung allokative Verbesserungen bewirkt.

Eine Kontingentierung der Externalitäten verursachenden Güter nach einem persönlichen oder sachlichen Merkmal könnte ein im Vergleich zur Marktpreiskoordination verringertes Angebot umweltschädlicher Produkte und damit auch eine Reduktion externer Effekte zur Folge haben. Z.B. wäre es denkbar, die schäd1 ichen Abgasemissionen des Kfz-Verkehrs einzuschränken, indem die Bereitstellung von Kraftfahrzeugen auf die berufliche Nutzung beschränkt bliebe. Obschon ein solches Vorgehen im Hinblick auf die Verwirklichung des Umweltzieles durchaus positiv zu bewerten wäre, sprechen nicht nur ordnungspolitische Erwägungen sondern ebenso auch Effizienzgesichtspunkte gegen die Kontingentierung. Es fallen hohe Transaktionskosten - insbesondere Durchführungs - und Kontrollkosten - an, die neue Fehlallokationen herbeiführen, so daß ein negativer Gesamteffekt nicht ausgeschlossen werden kann.

1) Coase spricht in diesem Zusammenhang von der Wahl des "appropriate social arrangements"; vgl. R. Coase, The Problem of Social Cost, a.a.0., S. 18. 
Als eine weitere Alternative zur Marktpreiskoordination kommt das Angebot bis zur Sättigung in Betracht. Statt die mit externen Nachteilen behafteten Güter zu diskriminieren, werden nunmehr Substitutionsguter, die nur geringe oder keine Externalitäten aufweisen, bevorzugt bereitgestel1t. So wäre eine Reduktion negativer Effekte des Individualverkehrs dann zu erwarten, falls öffentliche Verkehrsleistungen zum Nulltarif angeboten wïrden. Ob und inwieweit die externen Nachteile tatsächlich eingedämmt würden, das hinge dann allein von der stärke des Substitutionseffektes ab. Dieser wird jedoch nicht ausschließlich durch den Preisvorteil öffentlicher Verkehrsleistungen bestimmt, sondern ist $u$. a. auch von der Fahrzeit und der Bequemlichkeit im Vergleich zum Individualverkehr abhängig. 1) Besteht Unsicherheit dariber, ob allokative Verbesserungen bewirkende Substitutionsprozesse überhaupt in nennenswertem Umfang einsetzen, so ist darüber hinaus der Tatsache Rechnung zu tragen, daB bereits die Möglichkeiten zur Bereitstellung von Substitutionsgütern beschränkt sind. AuBerdem dürte die Koordination der Güter und Dienste über ein Angebot bis zur Sättigung für die Volkswirtschaft kaum weniger Kosten verursachen als die Kontingentierung. Zwar fallen die Transaktionskosten insgesamt niedriger aus, da - anders als bei der Kontingentierung - auf intensive Kontrollmaßnahmen verzichtet werden kann, dafür wird aber infolge der ausgeweiteten Güterbereitstellung gegen ein nur geringes Entgelt die Belastung für die öffentlichen Haushalte ungleich höher anzusetzen sein.

1) Gerade diese Faktoren potentieller Substitutionseffekte werden allerdings nicht selten durch die Schaffung dubioser Konkurrenzsituationen seitens der öffentlichen Planung konterkariert, z. B. indem parallel $z u$ öffentlichen Verkehrslinien ein Neu- oder Ausbau von Zubringerstraßen erfolgt; vgl. dazu auch die Ausführungen zu Punkt 1.2. 
Da weder vom Windhundverfahren noch von der Verlosung bessere Resultate zu erwarten sind, ist als weiteres Zwischenergebnis zu vermerken, daB - ähnlich wie die Ausweitung des Marktpreissystems als Strategie zur Internalisierung externer Effekte auch die Ablösung des Marktpreissystems durch nichtmarktliche Koordinationsmechanismen lediglich in Ausnahmefällen als ein geeigneter Ansatz zur Lösung des Externalitätenproblems zu werten ist.

Jenseits der beiden Extreme Marktausweitung und Marktablösung sind $z$ wei weitere Alternativen zur Behandlung externer $\mathrm{Nach}-$ teile zu unterscheiden, und zwar zum einen statiche Interventionen in den Marktpreismechanismus zur Korrektur der durch externe Effekte verzerrten Knappheitsrelationen und zum anderen Maßnahmen des Staates zur Heilung der durch die externen Nachteile hervorgerufenen Schäden, ohne daB Eingriffe in die Marktpreiskoordination selbst vorgenommen werden. Im Rahmen der Umweltpolitik sind die beiden Ansätze unter den Stichworten Verursacherprinzip und Gemeinlastprinzip bekannt. 1)

Nach dem Gemeinlastprinzip wird der Umweltschutz als rein staatliche Aufgabe betrachtet, d.h. der Staat ergreift in eigener Regie Maßnahmen zur Verringerung von Umweltschäden (z.B. Bau von Kläranlagen, Errichtung von Schallschutzwänden). Die Anwendung des Gemeinlastprinzips ist immer dann unumgänglich, wenn die Verursacher externer Nachteile nicht belangt werden können ${ }^{2}$, erweist sich aber auch dann als zweckmäBig, falls der Spielraum zur Reduktion einer umweltschädlichen Handlung gering ist ( $z$. B. Abwässer privater Haushalte) und gleichzeitig Vorteile der Kostendegression genutzt werden können, wenn sich zeigt, daB groBe von Kommunen oder überörtlichen Trägern betriebene Kläranlagen kostengünstiger arbeiten als

1) Uberblickartig dazu: Der Rat von Sachverständigen für Umweltfragen, Umweltgutachten 1974, BT-Drucksache $7 / 2802$ vom 14.11.1974, Ziff. 565 ff.

2) Dies ist etwa der Fall, wenn die Umweltschädigung lange zurückliegt und deshalb die Verursacher nicht mehr zweifelsfrei zu identifizieren sind oder falls es sich um Verursacher im Ausland handelt, gegen die keine rechtlichen Durchgriffsmöglichkeiten bestehen. 
kleinere, von einzelnen Wirtschaftssubjekten installierte Vorrichtungen zur Abwasserreinigung. Ein schwerwiegender Nachteil des Gemeinlastprinzips besteht hingegen nicht nur in der hohen finanziellen Belastung der öffentlichen Haushalte, sondern vor allem in dem Verzicht auf Internalisierung der Externalitäten. Das Problem der Fehlallokation wird nicht gelöst, da auf seiten der Verursacher keine Anreize zur Reduktion umweltschädlicher Emissionen bestehen.

Ohne Zweifel stellt die Orientierung am Verursacherprinzip die marktkonforme Interventionsvariante dar. Der Leitgedanke besteht darin, die durch externe Effekte verzerrten Marktpreise zu korrigieren, indem die volkswirtschaftlichen Kosten der Umweltschäden dem Verursacher dieser Schäden angelastet werden. Hier$z u$ bietet sich der Einsatz verschiedener staatlicher Instrumente an. So kann die Erhebung von Abgaben beim Verursacher allein aus Rentabilitätsüberlegungen die Motivation zur Reduktion von Umweltschäden wecken, während durch Auflagen oder dem schlichten Verbot bestimmter irreparabler Umweltschädigungen der Verursacher zur Vornahme von Vermeidungsinvestitionen gezwungen wird. Abgaben, Auflagen oder Verbote erhöhen die Kosten umweltbelastender Produkte oder Produktionsverfahren, bewirken mithin einen Preisanstieg dieser Produkte und induzieren damit - jedenfalls idealiter - gewünschte Veränderungen der Angebots - und Nachfragestruktur. ${ }^{1)}$ Obschon das Verursacherprinzip im Hinblick auf die allokative Effizienz dem Gemeinlastprinzip im allgemeinen überlegen sein dürfte, hat der Sachverständigenrat für Umweltfragen mit Nachdruck betont, da $B$ die direkte politische Umsetzung eines idealtypischen Verursacherprinzips wegen des hohen Informationsbedarfes und

1) Zur allokativen Beurteilung der dem Verursacherprinzip folgenden Instrumente $\mathrm{vgl}$. insbesondere K. Littmann, Umweltbelastung - Sozialökonomische Gegenkonzepte, a.a.0., S. $61 \mathrm{ff}$.; H. Siebert, Analyse der Instrumente der Umweltpolitik, Göttingen 1976; Der Rat von Sachverständigen für Umwel tfragen. Umwel tgutachten 1978, Stuttgart und Mainz 1978, Ziff. $1691 \mathrm{ff}$. 
aufgrund mangelnder Praktikabilität scheitern muß. ${ }^{1}$ ) Er empfiehlt deshalb als wesentlich pragmatischere Vorgehensweise die am Einzelfall vorzunehmende Zweckmäßigkeits- und Effizienzprüfung des verursacherorientierten Instrumentariums und darüber hinaus die Berücksichtigung der Rahmenbedingungen und Durchsetzungsmöglichkeiten des Verursacherprinzips sowie der potentiellen oder auch tatsächlichen Widerstände bei der Anwendung der Instrumente. ${ }^{2)}$

Die externen Effekte der Produktion sind wie kaum ein zweites ökonomisches Phänomen geeignet, auf die breite Palette der Varianten wirtschaftspolitischer Aufgabenverteilung aufmerksam zu machen. Wie gezeigt, bieten sich folgende Interventionsebenen an:

- Schaffung gesetzlicher Rahmenbedingungen als Voraussetzung für eine Ausweitung der Marktpreiskoordination auf Umweltgüter;

- Obernahme des Umweltschutzes als staatliche Aufgabe bei unveränderter Marktpreiskoordination der mit externen Nachteilen behafteten Güter und Dienstleistungen;

- Einsatz staatlicher Instrumente zur Korrektur der Marktpreis koordination sowie

- Ablösung des Marktpreissystems und Bereitstellung der mit externen Nachteilen behafteten Güter und Dienstleistungen über nichtmarktliche Koordinationsmechanismen.

Obschon einige grundlegende Erwägungen dafür sprechen, dem Einsatz staatlicher Instrumente zur Korrektur der Marktergebnisse den Vorrang einzuräumen, bleiben doch situationsbezogene Entscheidungen unerläßlich. Der Entscheidungsprozeß hat dabei in mehreren Schritten zu erfolgen:

1) $\mathrm{Vgl}$. Rat von Sachverständigen für Umweltfragen, Umweltgutachten 1978, a.a.0., Ziff. 1693.

2) Ebenda, Ziff. 1757; zủ den Grenzen der konkreten Anwendbarkeit des Verursacherprinzips vgl. auch K.-H. Hansmeyer, Die Abwasserabgabe als Versuch einer Anwendung des Verursacherprinzips, in: OKkonomische Probleme der Umweltschutzpolitik, hrsg. von 0. Issing, SchdVSocpol, N.F., Bd. 91, Berlin 1976, S. $65 \mathrm{ff}$. 
1. Mit Hilfe empirischer Untersuchungen ist die Relevanz externer Effekte zu analysieren, d.h. es ist das Ausmaß der durch Externalitäten verursachten Fehlallokationen und damit die Höhe der für die Volkswirtschaft entstandenen sozialen Kosten zu ermitteln.

2. Die potentiellen Interventionsalternativen zur Vermeidung oder Beseitigung der externen Nachteile zeichnen sich durch erhebliche Kostenunterschiede aus; während die direkten und indirekten Interventionskosten relativ exakt zu berechnen sind, können die Transaktionskosten sowie etwaige soziale Kosten der Intervention aufgrund der Quantifizierungsprobleme nur grob abgeschätzt werden.

3. Den durch externe Nachteile bedingten volkswirtschaftlichen (sozialen) Kosten sind die Gesamtkosten der Reduktion von Externalitäten gegenüberzustellen. Das ökonomische Entscheidungskriterium unter dem Blickwinkel der allokativen Effizienz lautet dann: Es ist diejenige wirtschaftspolitische Interventionsform zu wählen, bei der die Summe aus den sozialen Kosten der Umweltschädigung und den Kosten des Umweltschutzes ein Minimum wird.

4. Es ist darüber hinaus stets zu prüfen, ob eine einzelne Interventionsform ( $z . B$. Abwasserabgabe) oder Kombinationen verschiedener Interventionsformen (z.B. die Errichtung staatlicher Kläranlagen gekoppelt mit einer Erhebung von Abgaben) unter der allokativen Zielsetzung günstiger zu beurteilen sind. 
2. Marktversagen auf der Nachfrageseite

2.1. Offentliche Güter

Allokatives Marktversagen wird - insbesondere in der finanzwissenschaftlichen Literatur - auch mit der Existenz öffentlicher Güter begründet. Deren gutsspezifische Eigenschaften seien verantwortlich für die Verletzung des Grundprinzips der Marktpreiskoordination, nach dem die Leistung stets vom Zustandekommen einer Gegenleistung abhängt (do-ut-des-Regel). Die fehlende Möglichkeit des Ausschlusses bei Nichtzahlung sowie die Nichtrivalität im Konsum bewirkten ein Free-Rider-Verhalten der Wirtschaftssubjekte mit der Folge, daß die Konsumentenpräferenzen nicht in Nachfrage am Markt umgesetzt würden. Obschon Bedarf vorhanden sei, bestuinde für private Anbieter deshalb auch keinerlei Anreiz, öffentliche Güter über den Markt bereitzustellen. Die Versorgung mit öffentlichen Gütern müsse daher vom Staat uibernommen werden. 1 )

Die Anmerkungen $z u$ den als konstitutiv erachteten Eigenschaften öffentlicher Güter im deskriptiven Teil dieser Arbeit ${ }^{2}$ ) haben freilich bereits gezeigt, daß Güter nicht "aus ihrem Wesen" heraus eindeutig und allgemein guiltig zu bestimmen sind. Folglich ist auch nicht a priori festzulegen, in welchen Fällen die Versorgung mit Gütern und Dienstleistungen öffentlich erfolgen muß. Vielmehr ist im Zuge technologischer und institutioneller Veränderungen auch der Gutscharakter Wandlungen unterworfen, d.h. es ergeben sich Gestaltungsmöglichkeiten für die Wirtschafts- und Gesellschaftspolitik, so daß eine Entscheidung darüber herbeizuführen ist, ob ein Gut öffentlich oder privat bereitgestellt werden soll.

Das Konzept der öffentlichen Güter kann somit zwar als notwendiges ökonomisches Beurteilungskriterium zur Feststellung eines allokativen Marktversagens den Ausgangspunkt der Analyse

1) Ursprünglich so auch R. A. Musgrave, The Theory of Public Finance, a.a.0., S. 8 .

2) Vgl. zweiten Teil, Abschnitt III, Punkte 1.2 und 1.3. 
bilden, ob und inwieweit aber tatsächlich ein Interventionsbedarf zu begründen ist, welche Interventionsformen prinzipiell denkbar sind und welcher Koordinationsmechanismus unter welchen Bedingungen Effizienzvorteile erbringt, das bedarf auch hier der Prüfung anhand hinreichender Entscheidungskriterien.

Gilt es als empirisch gesichert, daß der AusschluB von Konsumenten nur in den seltensten Fällen technisch völlig unmöglich ist, so rücken die Kosten des Ausschlusses in den Blickpunkt des Interesses. Die ökonomische Entscheidungsfrage lautet dann: Wann ist es effizient, das Ausschlußprinzip anzuwenden? Bei oberflächlicher Betrachtung scheint eine eindeutige Antwort rasch gefunden: Je höher die AusschluBkosten, desto größer ist ceteris paribus der Effizienzvorteil einer auf den Ausschluß nichtzahlender Konsumenten verzichtenden Güterkoordination. Eine genauere Analyse zeigt indes, daB die Höhe der Ausschlußkosten nicht in jedem Fall darüber Auskunft gibt, ob ein. Ausschluß effizient ist oder nicht.

So verursachen z.B. die Tätigkeit der Kassenangestellten und die Aktivität des Hausdetektivs in einem Kaufhaus zwar beträchtliche Ausschlußkosten, ohne daß damit aber Effizienzeinbußen verbunden sind. Dagegen bewirken selbst vergleichsweise geringe Ausschlußkosten immer dann Effizienzverluste, falls die Nichtrivalität im Konsum derart ausgeprägt ist, daß die Befriedigung eines weiteren Nachfragers keine zusätzlichen Kosten verursacht (z.B. die Benutzung einer nicht überfüllten Brücke), mithin die Grenzkosten der Konsumentenbeteiligung gleich Null sind und daher gemäß den Regeln der Allokationseffizienz die Güterbereitstellung zum Preis von Null erfolgen müßte. 1) Neben den Ausschlußkosten sind deshalb auch jene gesamtwirtschaftlichen Kosten zur Effizienzbeurteilung heranzuziehen, die - abhängig vom Grade der Rivalität - durch Nachfrageänderungen bedingt sind. ${ }^{2)}$

1) So auch R. A. Musgrave, Provision for Social Goods in the Market System, a.a.0., S. 307 .

2) So auch J. G. Head und C. S. Shoup, Public Goods, Private Goods, and Ambiguous Goods, in: Economic Journa 1, Bd. 79 (1969), S. 567 f. sowie dieselben, Public, Private, and Ambiguous Goods Reconsidered, in: Public Finance, Bd. 28 (1973), S. 386. 
Allgemein gilt, daß bei ausgeprägter Nichtrivalität mit dem Verzicht auf Ausschluß zwar eine Nachfrage-, nicht aber eine Kostenerhöhung einhergeht. Die Anwendung des Ausschlußprinzips ist in diesen - freilich seltenen - Fällen ineffizient. Bewirkt der Verzicht auf Ausschluß jedoch - wie in der überwiegenden Zahl der Fälle - nicht nur eine Nachfrage- sondern auch eine Kostenerhöhung, dann sind die Einsparungen durch den Fortfall der Ausschlußkosten abzuwägen gegen die zusätzlich auftretenden Kosten der Nachfrageausweitung. Beide gesamtwirtschaftlichen Kostenfaktoren, zwischen denen mithin eine Trade-off-Beziehung besteht, können - da sie unmittelbar im Zuge der Betreibung von Koordinationsmechanismen anfallen - als Transaktionskosten bezeichnet werden.

überdies ist auf einen Punkt aufmerksam zu machen, der bisher weitgehend vernachlässigt wurde. Allgemein wird aus der Anwendung des Ausschlußprinzips gefolgert, die Güterbereitstellung werde uber den Marktpreis gesteuert, d.h. die Praktizierung des Ausschlusses wird mit Marktausschluß gleichgesetzt. 1) Mit der Anwendung des Ausschlußprinzips ist jedoch nicht bereits automatisch die Entscheidung zugunsten des Marktpreissystems gefallen. Vielmehr wird ein Ausschluß auch bei den nichtmarktlichen Koordinationsmechanismen Kontingentierung, Windhundverfahren und Verlosung praktiziert. Vom Marktpreissystem unterscheiden sich diese Mechanismen im Hinblick auf den AusschluB lediglich durch das Ausschlußverfahren.

Werden die obigen überlegungen in die Analyse einbezogen, so ist die Entscheidungsfrage nochmals wie folgt zu präzisieren: In welchen Fällen ist unter Berücksichtigung der Transaktions-

1) Dies gilt auch für Head und Shoup, deren Anliegen es ist, zu zeigen, in welchen Fällen die Anwendung des Ausschlußprinzips im Vergleich zum Verzicht auf Ausschluß unter Finanzierungsgesichtspunkten die effizientere Alternative darstellt. Der Ausschluß ist bei innen identisch mit Marktpreisfinanzierung (marketing mode), während der Nichtausschluß der Budgetfinanzierung (non-marketing mode) entspricht. Vgl. J. G. Head und C.S. Shoup, Publ ic Goods, Private Goods, and Ambiguous Goods, a.a.0., S. 567, prazisierend dieselben, Public, Private, and Ambiquous Goods Reconsidered, a.a.0., S. 385 . 
kosten ${ }^{1)}$ das Angebot von Gütern und Dienstleistungen über einen Koordinationsmechanismus, dem das Ausschlußprinzip zugrundeliegt, effizienter als die Güterbereitstellung über einen Mechanismus ohne Anwendung des AusschluBprinzips?

Fünf Fälle sollen im folgenden kurz illustriert werden:

Erster Fall: Weisen Güter eine ausgeprägte Nichtrivalität auf, so können die Grenzkosten der Konsumentenbeteiligung über weite Strecken Null sein (Beispiel der Brücke, solange keine Oberfiillung eintritt). Erfolgt kein AusschluB und fallen damit auch keine Ausschlußkosten an, so werden die Transaktionskosten im oben definierten Sinn ebenfalls Null. Folglich ist es gesamtwirtschaftlich ineffizient, das Ausschlußprinzip anzuwenden, und zwar unabhängig von der Höhe der AusschluBkosten und unabhängig von dem gewählten Ausschlußverfahren. Die öffentliche Bereitstellung über ein Angebot bis zur Sättigung ist daher in diesem Fall der gesamtwirtschaftlich effiziente Koordinationsmechanismus.

Zweiter Fall: Unter den im ersten Fall genannten Bedingungen ist eine Konstellation denkbar, die - selbst bei strenger Beachtung der Effizienz - nicht notwendigerweise eine öffentliche Güterversorgung erforderlich macht. Ein privates Angebot ist dann zu erwarten, falls der Nutzen, den ein einzelnes Individuum oder eine Gruppe aus dem Konsum eines bestimmten Gutes zieht, die Bereitstellungskosten dieses Gutes übertrifft. Gängige Beispiele hierfür sind das private Feuerwerk oder die private Gartenanlage. Flankierende staatliche Interventionen können hier positiv wirken, sofern sie darauf gerichtet sind, die Leistungsbereitschaft der Privaten zu erhöhen, indem u.a. die rechtlichen Voraussetzungen geschaffen oder finanzielle Hilfen gewährt werden ( $z$.B. im Hinblick auf den Bau und die Beaufsichtigung eines Kinderspielplatzes in Eigeninitiative der Gemeindebewohner).

1) Zu den Transaktionskosten gehören allerdings nicht nur die oben genannten Kostenfaktoren, sondern ebenso weitere Kosten der Durchführung sowie Kosten zur Herstellung und Kontrolle des Koordinationsprozesses; vgl. dritten Teil, Abschnitt II, Punkt 3.3. dieser Arbeit. 
Dritter Fall: Sind die Ausschlußkosten hoch und herrscht partielle Rivalität im Konsum ${ }^{1)}$, dann kann die Höhe der Transaktionskosten je nach Wahl des Koordinationsverfahrens stark variieren. Während bei der Marktlösung die Ausschlußkosten voll zu Buche schlagen, sind sie bei einem Angebot bis zur Sättiquna Nul1. Dafür muissen die infolge des Verzichts auf AusschluB zusätzlich anfallenden gesamtwirtschaftlichen Kosten der Nachfrageausweitung in Rechnung gestellt werden. Bei der Kontingentierung, dem Windhundverfahren und der Verlosung sind die Ausschlußkosten unterschiedlich hoch, auf jeden Fall aber positiv. Allgemeingültige Aussagen über etwaige kostenverursachende Nachfrageänderungen sind hier nicht möglich, sie häncen vielmehr von der konkreten Ausgestaltung des jeweiligen Koordinationsmechanismus ab. Die Kosten können ähnlich hoch ausfallen wie beim Angebot bis zur Sättigung, andererseits sind aber auch gesamtwirtschaftliche Kostenersparnisse gegenuber der Marktpreiskoordination nicht ausgeschlossen.

Die Formulierung einer Effizienzregel wird durch einen weiteren Umstand erschwert. Weder die Höhe der Ausschlußkosten noch der Rivalitätsgrad besitzen den Charakter eines Datums, sondern sind sowohl durch Aktivitäten im privaten Sektor als auch durch Interventionen des öffentlichen Bereichs variierbar. So können die Ausschlußkosten z.B. durch technischen Fortschritt (z.B. Leuchtturm mit Störungs-Entstörungsvorrichtungen) gesenkt werden. Ceteris paribus verbessert sich die Effizienz des Ausschlusses, d.h. Koordinationsmechanismen mit Anwendung eines Ausschlußverfahrens können nun gegenüber dem Angebot bis zur Sättigung Effizienzgewinne verzeichnen. Mit zunehmender Nachfrage vermindert sich häufig die Qualität eines angebotenen Gutes. Typische Beispiele sind Parkanlagen und Straßen. Treten Ballung, Warteschlangen und Umweltverschmutzung auf ${ }^{2}$, so entwickeln sich aus der ursprünglichen Nichtrivalität nun u.U.

1) Vgl. dazu zweiten Teil, Abschnitt III, Punkt 1.2.

2) Vgl. A. C. Deserpa, a.a.0., S. $68 \mathrm{ff}$. 
sehr ausgeprägte Rivalitätsbeziehungen zwischen den Konsumenten. Die Anwendung des Ausschlußprinzips verspricht dann auch hier gesamtwirtschaftliche Effizienzgewinne.

Inwieweit aber der Marktausschluß effizientere Resultate als andere Ausschlußverfahren gewährleistet, hängt zum einen von der Höhe der übrigen Transaktionskosten (Informations-, Durchführungs- und Kontrollkosten), zum anderen von den Kosten der dann erforderlichen statlichen Interventionen in den Marktpreismechanismus ab. Ohne flankierende Maßnahmen wird ein ursprüaliches Versagen des Marktes auf der Nachfrageseite u.U. ledialich in ein neues, durch Monopolbildungen, sinkende Durchschnittskosten oder externe Effekte hervorgerufenes Marktversagen auf der Angebotsseite transformiert.

Vierter Fall: Bisher wurde ausschließlich auf der Güterebene argumentiert, d.h. Güter wurden als Produktions- und Dienstleistungsergebnisse aufgefaßt und nicht funktional definiert. Die Effizienzbetrachtungen werden erschwert, sobald der Zusammenhang zwischen Güterbereitstellung und Aufgabenerfüllung heraestellt wird. 1) Ein Beispiel mag den Sachverhalt wiederum verdeutlichen. Eine Parkanlage stellt nicht nur ein Erholungsangebot für die Besucher dar, sondern sie dient gleichzeitig auch der Reinhaltung der Luft. Das Gut Parkanlage erfiult mithin zwei ganz verschiedene Aufgaben: die (private) Aufgabe "Erholung für Parkbesucher", auf die das Anschlußprinzip ohne Schwierigkeiten anzuwenden ist, und die (öffentliche) Aufaabe "Reinhaltung der Luft", die eine effiziente Praktizierung des Ausschlußprinzips bei dem gegenwärtigen Stand der Technik und den gegebenen institutionellen Bedingungen nicht zuläßt. Wir haben es hier mit Gütern zu tun, die eine effiziente Anwendung des Ausschlußprinzips nur auf Teile, nicht aber auf die gesamte Nutzenstiftung erlauben.

Fünfter Fall: Ist es denkbar, daß ein einzelnes Gut der Erfüllung zweier (oder auch mehrerer) ganz unterschiedlicher Aufgaben dient, so ist es umgekehrt ebenso möglich, daß dieselbe

1) Vgl. den zweiten Teil, Abschnitt III, Punkt 1.4 dieser Arbeit. 
Aufgabe mit Hilfe verschiedener "privater" und "öffentlicher" Guiter wahrgenommen wird. So kann z.B. ein verbesserter schutz vor Einbruch in einem Stadtgebiet grundsätzlich entweder durch den Einsatz von Polizeistreifen oder durch die Installation von Haussicherungsanlagen erreicht werden. Hier gilt es, gleich eine doppelte Frage zu beantworten, nämlich welche Form der Aufgabenerfüllung führt über welchen koordinationsmechanismus zur effizienten Ressourcenallokation?

Werden zum Schutz vor Einbruch Polizeistreifen eingesetzt, so bleiben aufgrund der ausgeprägten Nichtrivalität im Konsum die Kosten der Aufgabenerfiillung - in einer beachtlichen Bandbreite - unbeeindruckt davon, wieviele Einwohner des Stadtgebietes die Dienste der Polizei in Anspruch nehmen. 1) Entstehen die Kosten aber insoweit unabhängig von der Zahl der Nachfrager, so ist die Anwendung des Ausschlußprinzips ineffizient. Deshalb ist im Hinblick auf den Einsatz von Polizeistreifen das Angebot bis zur Sättigung der Koordinationsmechanismus der Wahl.

Aufgrund der Rivalität im Konsum und der problemlosen Anwenduna des Ausschlußprinzips ist eine effiziente Bereitstellung von Haussicherungsanlagen über den Marktpreismechanismus möglich. Würde hingegen politisch entschieden, daß Haussicherungsanlagen in jedem Haushalt des Stadtgebietes unentgeltiich zu installieren sind (Angebot bis zur Sättigung), so würden damit zwar die ohnehin nicht effizienzschädlichen Ausschlußkosten auf Null gesenkt, gleichzeitig aber würde - infolge des Free-Rider-Verhaltens der Wirtschaftssubjekte - die Nachfrage nach den zum Nullpreis angebotenen Haussicherungsanlagen im Verqleich zur Marktnachfrage höher ausfallen. Deshalb führt der Verzicht auf AusschluB und das damit korrespondierende Mitnahmeverhalten der Wirtschaftssubjekte zweifellos zu einer höheren Belastung der Volkswirtschaft mit gesamtwirtschaftichen Kosten. 2 )

1) Anders als im dritten Fall geher, wir hier wieder - wie bereits im vierten Fall - von einem gegebenen Stand der Technik aus.

2) Außerdem fallen zusätzliche Kosten an, da auch mit der Installation von Haussicherungsanlagen weiterhin polizeiliche Bereitschaftsdienste erforderlich sind. 
Alle bisherigen Oberlegungen münden freilich lediglich in ein kaum überraschendes Zwischenergebnis: Die Installation von Haussicherungsanlagen ist dann effizient, wenn sie über den Marktpreismechanismus erfolgt, während der mittels Polizeistreifen zu erzielende Schutz vor Einbruch effizient über ein Angebot bis zur Sättigung vorgenommen werden kann. Ob die öffentliche Aufaabe "Schutz vor Einbruch" aber unter dem Aspekt der Allokationseffizienz besser über den Markt oder über die öffentliche Alternative zu erfüllen ist, kann ohne zusätzliche Kriterien nicht entschieden werden. Aufzunehmen sind insbesondere Hypothesen über das Nachfrageverhalten der Individuen.

\subsection{Meritorische Güter}

Werden im Hinblick auf öffentliche Güter statliche Interventionen damit begründet, daß infolge des Free-Rider-Verhaltens der Konsumenten die Umsetzung der individuellen Präferenzen in Marktnachfrage unterbleibt, so erfolgt die Rechtfertiguna staatlicher Aktivitäten auch noch mit einem zweiten Argument: Gewisse Güter und Dienstleistungen werden zwar von den Konsumenten nachgefragt, die Entfaltung dieser Nachfrage sei allerdings "fehlerhaft", sie falle bei einigen Gütern zu gering, bei anderen zu hoch aus.

MUSGRAVE bezeichnet diese Güter als meritorische bzw. demeritorische Güter und führt als Beispiele für die erstaenannte Kategorie vom öffentlichen Verband bereitgestelltes Schulfrühstück, Wohnungsbauzuschüsse sowie Lern- und Lehrmittelfreiheit an. 1) Als demeritorische Güter können entsprechend Alkohol, Tabak und Rauschgifte genannt werden. Meritorische Güter sind nach MUSGRAVE folgendermaßen zu kennzeichnen: Das Ausschluß- und das Rivalitätsprinzip sind zumindest teilweise, wenngleich nicht immer auf den Gesamtnutzen, effizient anwendbar. Demzufolge werden meritorische Güter zwar in den durch die effektive Nachfrage gesteckten Grenzen über den Markt bereitgestellt, jedoch als so bedeutend angesehen, daß für sie durch das öffentliche Budget über

1) Vgl. R. A. Musgrave, The Theory of Public Finance, a.a.0., S. 13. 
den Rahmen des Marktangebotes und der Zahlungsbereitschaft der Konsumenten hinaus Sorge zu tragen ist. 1 )

Wie kaum ein zweiter finanztheoretischer Ansatz hat das Konzept der meritorischen Güter Verwirrungen und kontroverse Diskussionen in der wissenschaftlichen Literatur ausgelöst. Zum einen dürften hierfür wohl die nicht immer eindeutican und deshalb Interpretationsversuche provozierenden Ausführungen MUSGRAVES selbst verantwortlich sein. Bedeutsamer ist aber zum anderen der Umstand, daß dieses Konzept einen Fremdkörper in der traditionellen, individualistisch fundierten Nutzentheorie bildet. Die Präferenzen der Konsumenten sind statt - wie vom okonomen gewohnt - als exogene Größen zu behandeln, nun als Variablen eines für Demokratien typischen, d.h. durch gesellschaftliche Gruppen und Institutionen genauso wie durch politische Führuna beeinfluBten fortlaufenden Wertbildungsprozesses in der ökonomischen Analyse zu berücksichtigen.

Es ist nicht erforderlich, die Diskussion im einzelnen nachzuzeichnen ${ }^{2)}$, sondern es genügt, sie insoweit zu berücksichtigen, wie sie zur Beantwortung folgender Fragen beiträgt:

- Lassen sich aus dem Konzept der meritorischen Güter ökonomische Beurteilungskriterien für ein Versagen des Marktes ableiten?

1) Vgl. R. A. Musgrave, The Theory of Public Finance, a.a.0., S. 13.

2) Vgl. dazu J. G. Head, On Merit Goods, in: Finanzarchiv, N.F., Bd. 25 (1966), S. T ff.; Ch. E. Mc Lure, Merit Wants: a Normatively Empty Box, in: Finanzarchiv, N.F., Bd. 27 (1968), S. $474 \mathrm{ff}$.; J. G. Head, Merit Goods Revisited, in: Finanzarchiv, N.F., Bd. 28 (1969), S. $214 \mathrm{ff}$.; N. Andel, Zur Diskussion über Musgraves Begriff der "merit wants", in: Finanzarchiv, N.F., Bd. 28 (1969), S. 209 ff.; D. A. L. Auld und P. C. Bing, Merit Wants: A Further Analysis, in: Finanzarchiv, N.F., Bd. $30^{\circ}$ $(197 / / 72)$, S. $257 \mathrm{ff}$.; A. G. Pulsipher, The Properties and Relevancy of Merit Goods, in: Finanzarchiv, N.F., Bd. $30(1971 / 72)$, S. 266 ff.; F. Buttler, Explikative und normative Theorie der meritorischen Güter - eine Problemanalyse, in: Zeitschrift für Wirtschafts- und Sozialwissenschaften, Bd. 93 (1973), S. $129 \mathrm{ff}$.; C. Folkers, Meritorische Güter als Problem der normativen Theorie öffentTicher Ausgaben, in: Jahrbuch für Sozialwissenschaft, Bd. 25 (1974), S. 1 ff.; zusammenfassend dazu K. Schmidt, Kollektivbedürnisse und Staatstätigkeit, a.a.0., S. $9 \mathrm{ff}$. 
- Vorausgesetzt, ein Interventionsbedarf ist zu begruinden, welche Interventionsformen sind prinzipiell denkbar?

- Ist mit Hilfe ökonomischer Entscheidungskriterien feststellbar, ob die Meritorisierung bestimmter Guiter anderen Interventionstechniken uberlegen ist und welche Formen der Meritorisierung (Beibehaltung von Marktlösungen oder Obergang zu nichtmarktlichen Koordinationsverfahren) gegebenenfalls zu empfehlen sind?

Ein Versuch zur Unterstützung des Musgrave'schen Konzepts wurde von HEAD unternommen. Er rechtfertigt staatliche Eingriffe in die Marktpreiskoordination in Form der "Meritorisierung" auf dreifache Weise: ${ }^{1)}$

1. Unvollständige und irreführende Informationen am Markt einerseits sowie irrationales Verhalten der Nachfrager andererseits bewirken eine Verzerrung der individuellen Präferenzen. Es ist deshalb eine legitime wirtschaftliche Aufgabe des Staates, eine Korrektur der bekundeten Konsumentenpräferenzen vorzunehmen.

2. Das Marktpreissystem reagiert auf die kaufkräftige Nachfrage, ohne die Einkommenssituation der Konsumenten zu berücksichtigen. Die Existenz meritorischer Güter ist daher bereits als Instrument der Verteilungspolitik zu begrüden, verhindert eine Meritorisierung doch, daß der Konsum bestimmter Güter nur einkommensstarken Gruppen ermöglicht wird.

3. Es gibt Güter, die untrennbare private und öffentliche Gutseigenschaften aufweisen. Die Bereitstellung über den Marktpreis im Rahmen der effektiven Nachfrage führt deshalb zu einem im Vergleich zur effizienten Allokation zu niedrigen versorgungsniveau.

1) Vgl. J.G. Head, On Merit Goods, a.a.0., S. $3 \mathrm{ff}$. 
Weitgehende obereinstimmung besteht darin, daß aus allokativer Sicht die vermutete Verzerrung der individuellen Präferenzen als das zentrale Kriterium für meritorische Interventionen anzusehen ist. 1) Unvollständige Informationen bewirken, daß Individuen den Nutzen bestimmter Güter falsch einschätzen. Dies ailt insbesondere für solche Güter, deren Nutzen erst zu einem späteren Zeitpunkt sichtbar werden. Verläßt ein 15-Jähriger das Gymnasium, so duirfte er sich kaum der Tragweite seines Handelns bewußt sein. Es ist zu vermuten, daß er den Nutzen einer guten Schulausbildung infolge unzureichender Informationen unterbewertet.

Besonders häufig wird offenbar der Nutzen jener Güter falsch eingeschätzt, die zu einer Verminderung des Zukunftsrisikos beitragen. So wird z.B. auf vorbeugende Gesundheitsmaßnahmen oftmals ganz verzichtet oder Altersvorsorge auf freiwilliger Basis nicht selten nur in unzureichendem Maße betrieben. Oder: Ein Afrikareisender schließt zwar eine Reisegepäckversicherung ab, da hier sowohl die Höhe als auch die Zeitdauer des Risikos überschaubar ist, er hält dagegen eine Malaria-Schutzimpfung für nicht erforderlich. Im Hinblick auf die Einschätzung des Zukunftsrisikos ist darüber hinaus ein weiterer Aspekt von Bedeutung. Die Risikobereitschaft der Individuen ist unterschiedlich ausgeprägt. Selbst vollständige Information über die Gefahren bestimmter Handlungsweisen wird den Risikofreudigen nicht davon abhalten, die Wahrscheinlichkeit des Schadenseintritts für sich persönlich als äußerst gering einzustufen (z.B. der auf das Tragen eines Sturzhelms verzichtende Motorradfahrer). Der Konsum von Zigaretten, Alkohol, Rauschgiften etc. dürfte zumindest u.a. auch Ausfluß dieser individuell unterschiedlichen Risikobereitschaft sein. 2) Der Nutzen von Gütern kann schließlich auch auf-

1) So J. G. Head, Merit Goods Revisited, a.a.0., S. 214 f.; N. Andel, a.a.0., S. 211; K. Schmidt, Kollektivbedürnisse und Staatstätigkeit, a.a.0., S. 16 und A. G. Pulsipher, a.a.0., S. $269 \mathrm{ff}$.

2) Bereits aus diesem Grunde ist daher die Feststellung Heads, es sei schlicht irrationales Verhalten, wenn Individuen trotz vollständiger Information über die gesundheitlichen Gefahren des Konsums dieser Güter die "falsche" Entscheidung träfen, wenig glücklich; vgl. J. G. Head, On Merit Goods, a.a.0., S. 5 . 
grund irreführender Informationen falsch eingeschätzt werden. So rückt bei der Werbung die Information zugunsten plakativer und teils desinformativer Aussagen, mit deren Hilfe ein bestimmtes Produkt für den potentiellen Käufer attraktiv gemacht werden soll, in den Hintergrund.

Im Hinblick auf das Verteilungsargument wird verschiedentlich die Ansicht vertreten, HEAD überinterpretiere MUSGRAVE, da dieser dem verteilungspolitischen Argument keine wesentliche Bedeutung beigemessen habe. ${ }^{1)}$ ohne Frage stellen aber meritorische Eingriffe zum einen ein eigenständiges Instrument der Distributionspolitik dar ${ }^{2)}$, zum anderen dürfte zwischen den verzerrten Konsumentenpräferenzen infolge unvollständiger oder irreführender Informationen und der Zugehörigkeit dieser Konsumenten zu den unteren Einkommensklassen eine Korrelation bestehen. Beziehern höherer Einkommensklassen fällt es leichter, ihre Informationsdefizite zu überwinden als Empfängern relativ niedrigerer Einkommen. Wenn im folgenden ausschließlich die allokativen Implikationen meritorischer Interventionen analysiert werden, so dient dieses Vorgehen allein dem Zweck, die Allokationsprobleme klar herauszustellen.

1) So insbesondere N. Andel, a.a.0., S. 210 und A. G. Pulsipher, a.a.0., S. $271 \mathrm{f}$.

2) Das Gut Wohnen bietet ein typisches Beispiel für die Nennuna gleich mehrerer Gründe zur Rechtfertigung fördernder staatlicher Eingriffe. Während der Wissenschaftliche Beirat beim Familienministerium betont, die zu gering entfaltenden Präferenzen seien auf mangelnde Kenntnisse der positiven Wirkungen befriedigender Wohnverhältnisse zurückzuführen, sieht die Bundesregierung in ihrem achten Subventionsbericht den Zweck von Wohnaeldzahlungen darin, auch Haushalte mit niedrigem Einkommen in die Lage zu versetzen, die ihnen allein nicht zumutbaren Mieten aufzubringen. Val. Wissenschaftlicher Beirat für Familienfragen beim Bundesminister für Jugend, Familie und Gesundheit, Familie und Wohnen, AnaTysen und Empfehtungen zur Wohnungsversorgung der Familien und zur Förderung familiengerechten Wohnens in der Bundesrepublik Deutschland, Schriften des Bundesministers, Bd. 20, Stuttgart 1975, S. 42; Bundesministerium der Finanzen (Hrsg.), Bericht der Bundesregieruna über die Entwickluna der Finanzhilfen und Steuervergünstigungen für die Jahre 1979 bis 1982 gemäß $\S 12$ des Gesetzes zur Förderung der Stabilität und des Wachstums der Wirtschaft vom 8. Juni 1967 (Achter Subventionsbericht), Bonn 1981, S. 41. Val. hierzu auch K. Mackscheidt, Zur Kritik einiger Rechtfertioungsaraumente für staatliche Interventionen auf dem Wohnungsmarkt, in: Zeitschrift für Wirtschafts- und Sozialwissenschaften, 102. Jg. (1982), S. $113 \mathrm{ff}$. 
Was schließlich die letzte Begründungskategorie HEADS anlangt, so hat bereits die Erörterung im Zusammenhang mit den öffentlichen Gütern gezeigt, daß es kaum ein Gut gibt, dem nicht private und öffentliche Nutzenkomponenten zuqleich zugewiesen werden können. Die Verbindung von privaten und öffentlichen Gutseigenschaften kann deshalb nicht als Spezifikum meritorischer Güter herangezogen werden.

Obschon die Verletzung der Konsumentensouveränität weder im privaten Sektor noch im Bereich öffentlicher Aktivitäten etwas Ungewöhnliches darstellt ${ }^{1)}$, ist das Spezifische der meritorischen Güter, daß hier - anders als bei privaten und öffentlichen Gütern - der Eingriff des Staates in die Konsumentenpräferenzen zur Erfüllung öffentlicher Aufaaben zum eigentlichen Zweck erhoben wird. 2) Gleichzeitig liegt aber in der bewußten Korrektur der individuellen Präferenzen auch das Hauptproblem der meritorischen Güter. Zum einen harmoniert der Eingriff in die Konsumentensouveränität nur äußerst schlecht mit der traditionellen normativen Theorie, zum anderen stellt sich nun die Frage, wer denn eigentlich legitimiert sei und in welchem Umfang, die Präferenzen der Konsumenten zu korriaieren. Beide Problembereiche sind in der Literatur intensiv diskutiert worden und müssen deshalb nicht erneut aufgegriffen werden. ${ }^{3}$ )

Hier genügt folgende Anmerkung: Ein großer Teil der wirtschaftlichen Aktivitäten ist weder das alleinige Ergebnis individueller Präferenzen noch das bloße Resultat der überlegenen Einsicht

1) So betont Andel in Bezug auf die Bereitstellung öffentlicher Güter: "Da hier die tatsächlichen Präferenzen aus den bekannten Gründen (weitgehend) unbekannt sind, kann man nicht angeben, bis zu welchem Punkt eine Maßnahme wirklich im Hinblick auf die gegebenen individuellen Konsumentenpräferenzen (bei gegebener Kaufkraftverteiluna) erforderlich ist und wo die diese Konsumentenpräferenzen übermehende 'eigenmächtige' diktatorische Entscheidung einsetzt." N. Andel, a.a.0., S. 112.

2) Vgl. R. A. Musgrave, The Theory of Public Finance, a.a.0., S. 14.

3) Statt vieler vgl. K. Schmidt, Kollektivbeduirfnisse und Staatstätiakeit, a.a.0., S. 0 ff. sowie F.Buttler, a.a.0., S. $129 \mathrm{ff}$. 
politischer Handlungsträger. Vielmehr handelt es sich um einen dynamischen Prozeß gegenseitiger Beeinflussung von politischer Führung und aktiver offentlichkeit. In diesem Prozeß hat der nach Stimmenmaximierung strebende und sich folglich an der Wählermehrheit orientierende Politiker gemäß DOWNS genauso seinen Raum wie der von COLM zitierte Politiker, der im politischen Kampf um aute Lösungen seine Popularität aufs spiel setzt. ${ }^{1}$ ) Insoweit ist das Konzept der meritorischen Güter "ein partielles Zugeständnis an die Realität der Demokratie"2), und meritorische Eingriffe des Staates unterscheiden sich dann nicht von anderen wirtschaftspolitischen Interventionen.

Wird anerkannt, daß verzerrte Konsumentenpräferenzen infolge unvollständiger oder irreführender Informationen sowie einer ausgeprägten Bereitschaft, Zukunftsrisiken einzugehen, allokative Ineffizienzen bewirken, wobei die Enge dieses Zusammenhanges eine empirische Tatfrage ist, so bildet dieser Zusammenhang für die theoretische Analyse das - notwendige - Beurteilungskriterium für einen Interventionsbedarf.

Es ist zu prüfen, welche Interventionsformen zur Korrektur der Konsumentenpräferenzen prinzipiell geeignet erscheinen.

In Obersicht 22 sind die relevanten Interventionsebenen und - formen gegenüber verzerrten Konsumentenpräferenzen dargestellt. Sie verdeutlicht bereits dreierlei:

1) Vgl. G. Colm, National Goals Analysis and Marainal Utility Economics, a.a.0., S. 217.

2) S. Katterle, a.a.0., S. 18. 
Obersicht 22: Interventionsebenen und -formen bei verzerrten Konsumentenpräferenzen

\begin{tabular}{|c|c|}
\hline $\begin{array}{l}\text { INTERVENT IONSEBENE } \\
\text { "INSTRUMENTE" }\end{array}$ & 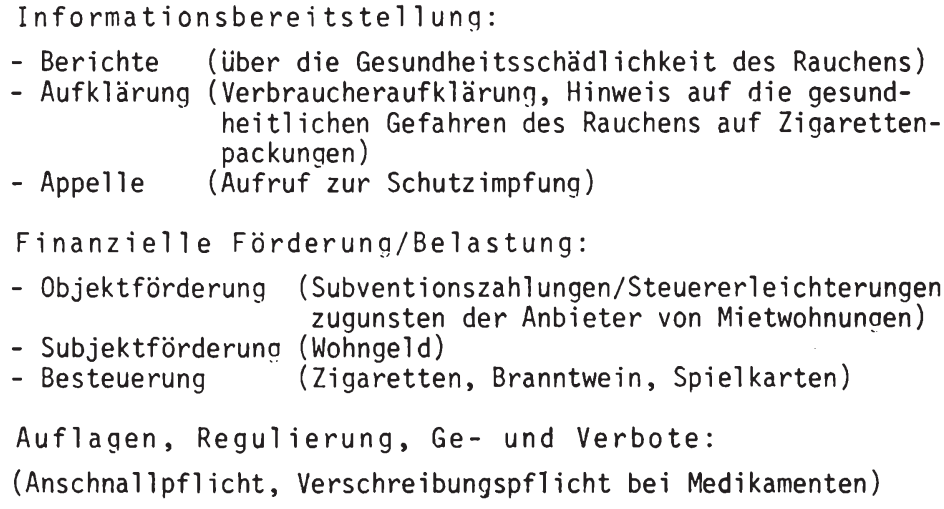 \\
\hline $\begin{array}{l}\text { INTERVENTIONSEBENE } \\
\text { "INSTITUTION" }\end{array}$ & $\begin{array}{c}\text { Verbände (Verbraucherschutzverband, Caritas) } \\
\text { Selbstorganisation (Altenhilfeverein, Initiativgruppe } \\
\text { für Nachhilfeunterricht) }\end{array}$ \\
\hline $\begin{array}{l}\text { INTERVENTIONSEBENE } \\
\text { "KOORDINATIONS- } \\
\text { MECHANISMEN" }\end{array}$ & $\begin{array}{l}\text { Kontingentierung: } \\
\text { - nach individuellem Merkmal (Sozialwohnungen) } \\
\text { Angebot bis zur Sättigung: } \\
\text { - mit Benutzungszwang (Schulbildung) } \\
\text { - ohne Benutzungszwang (Volkshochschulkurs) }\end{array}$ \\
\hline
\end{tabular}


- Staatliche Interventionen, die auf eine Korrektur der individuellen Präferenzen abzielen, sind nicht in jedem Falle gleichbedeutend mit einer Bereitstelluna meritorischer Güter. Eine Alternative zur Meritorisierung ist die Informationsbereitsteilung.

- Die Meritorisierung selbst kann wiederum sehr unterschiedliche Formen annehmen. Die Beibehaltung der Marktpreiskoordination ist dabei genauso denkbar wie die Bereitstellung über nichtmarktiche Koordinationsverfahren.

- Mit der Meritorisierung ist nicht automatisch eine Verminderung der Entscheidungsfreiheit der Individuen verbunden. Lediglich der Einsatz regulativer Instrumente oder das Angebot bis zur Sättigung mit Benutzungszwang führt zu einer mit physischen Mitteln durchsetzbaren Einschränkuna der individuellen Dispositionsmöglichkeit. ${ }^{1)}$ Daneben wird deutlich, daß die Intensität der Entscheidungseinschränkung nichts damit zu tun hat, ob eine Marktlösung beibehalten wird oder zu einem nichtmarktlichen Koordinationsmechanismus übergegangen wird. U. U. werden regulative Eingriffe in den Markt (z.B. Anschnalloflicht) intensiver empfunden als die öffentliche Güterbereitstelluna in Form eines Angebots bis zur Sättigung ( $z$. B. Schulpflicht).

Unter dem Gesichtspunkt der allokativen Effizienz sind vor allem drei alternative Interventionstechniken auszuleuchten:

- (a) Informationsbereitstellung versus Meritorisierung

- (b) Meritorisierung durch Objekt- versus Subjektförderung

- (c) Bereitstellung meritorischer Güter über den Marktpreis versus nichtmarktliche Güterbereitstellung.

1) Head vertritt deshalb die Ansicht, daß "only those measures which involve some form of 'physical compulsion' might be regarded as true merit goods policies." J.G.Head, Merit Goods Revisited, a.a.0., S. 219. Damit wird freilich erneut die von uns bereits bei anderer Gelegenheit kritisierte Verknüpfung von Aufgabenbestimmung und Art der Aufgabenerfuillung vorgenommen. A priori und ohne exakte Fallanalyse kann jedoch beispielsweise nicht geschlossen werden, die meritorische Aufgabe "Eindämmung des Alkoholkonsums" sei wirksamer durch ein Verbot als auf dem Wege einer Besteuerung zu erfuillen. Vielmehr handelt es sich zunächst um gleichwertige Alternativen der "merit goods policy". 
Zu (a): Im Hinblick auf die Interventionsalternative "informieren" oder "meritorisieren" hat MCLURE mit seinem eindeutigen Votum zugunsten der Informationspolitik die Diskussion angeregt: Werden unvollständige oder irreführende Informationen als Hauptursache verzerrter Konsumentenpräferenzen angesehen, so ist zur Korrektur dieser Präferenzverzerrungen konsequenterweise ausschließlich für eine gezielte öffentliche Informationstätiakeit, nicht aber für eine öffentliche Güterbereitstelluna zu plädieren. 1) Demgegenüber sieht HEAD die Beschränkung des öffentlichen Sektors auf eine bloße Informationstätigkeit als unzureichend an, da Individuen den Nutzen von Gütern erst durch deren Konsum kennenlernen, mit dem wünschenswerten Effekt, daß die Betroffenen nicht nur ex post den meritorischen Eingriff billigen, sondern darüber hinaus auch eine adäquate Marktnachfrage entfalten. ${ }^{2}$ )

Keines der beiden Argumente kann indes vollkommen überzeugen. Gegen eine reine Informationspolitik ist einzuwenden, daß - wie der Fall ausgeprägter individueller Risikobereitschaft zeigt - selbst be $i$ vollständiger Information Präferenzverzerrungen auftreten können. Zusätzliche Aufklärungsmaßnahmen bewirken dann keine Änderungen der Präferenzen. Zudem wird kritisiert, daß McLURE die Informationen als freies Gut behandelt. ${ }^{3}$ ) Wird realistischerweise davon ausgegangen, daß die Informationsbeschaffung und -verarbeitung Kosten verursacht, so handelt ein Individuum rational, wenn es sich nur bis zu einem gewissen Grade mit Informationen versorgt. Okonomisch exakter formuliert: Ein Wirtschaftssubjekt wird nur solange Informationen aufnehmen, solange die Grenzkosten den Grenznutzen der Information nicht übertreffen. Mit diesem Hinweis ist zwar das Problem erkannt, aleichwohl aber noch keine Lösung in Sicht. Die Abwägung von Grenznutzen und Grenzko-

1) $\mathrm{Vgl}$. Ch. E. McLure, a.a.0., S. 477.

2) $\mathrm{Vgl}$. J.G. Head, On Merit Goods, a.a.0., S. 5.

3) Vgl. A. G. Pulsipher, a.a.0., S. 271. McLure macht allerdings darauf bereits selbst aufmerksam; vgl. Ch. E. McLure, a.a.0., S. 477, Fußn. 2. 
sten setzt nämlich zwingend voraus, daß die Konsumenten in der Lage sind, den Nutzen der Information richtig einzuschätzen. Gehen wir davon aus, daß sie es nicht können, so haben wir es mit einem logischen Dilemma zu tun. "The difficult problem is to inform those who are unaware that they are uninformed."1) Positiver ausgedrückt: Die Informationsbereitstellung wird nun selbst zu einem meritorischen Gut.

Auf der Suche nach einem Entscheidungskriterium stellen AULD und BING ebenfalls die Informationskosten, allerdings die Kosten der Informationsbereitstellung, in den Vordergrund. Sie empfehlen eine Meritorisierung dann, wenn durch öffentliche Informationsbereitstellung mehr Ressourcen gebunden werden als durch den meritorischen Eingriff. 2 )

Zur Beantwortung der Entscheidungsfrage "informieren" oder "meritorisieren" werden somit auch hier die relativen Kosten der Intervention zum ausschlaggebenden Entscheidungskriterium erhoben. Freilich stoßen Kostenvergleiche bereits deshalb auf enae Grenzen, weil sich zwar die öffentlichen Informationsbereitstellungskosten überwiegend problemlos ermitteln lassen, die Feststellung auf seiten der Konsumenten jedoch Schwierinkeiten bereitet.

HEADS Argument, Individuen würden den Nutzen von Gütern erst durch deren Konsum kennenlernen, kann ebenfalls keine allgemeine Gültigkeit beanspruchen. Es gibt eine Reihe von Gütern, deren Nutzen nicht durch den Konsum erkannt wird, deren öffentliche Bereitstellung also nicht automatisch auch Lernprozesse auslöst (z.B. Schutzimpfung, Tragen von Sicherheitsgurten oder Schutzhelmen). Hier handelt es sich vorwiegend um präventiven Konsum

1) A. G. Pulsipher, a.a.0., S. 271.

2) In ihrer Untersuchung beschränken sie sich allerdinas auf den Fall der demeritorischen Guiter und kommen hier zu dem Ergebnis, daß unter Effizienzaspekten eine rein steuerliche Beeinflussung der Konsumentenentscheiduna einer staatlich finanzierten Aufklärungskampagne überlegen ist. Vgl. D. A. L. Auld und P. C. Bing, a.a.0., S. 261. Kritisch dazu M. Braulke, Merit Goods: Einige zusátzTiche Anmerkungen, in: Finanzarchiv, N.F., Bd. 31 $(1972 / 73)$, S. $307 \mathrm{ff}$. 
zur Verhütung von Nutzeneinbußen in der Zukunft. Diese Güter sind jedoch weitgehend identisch mit jenen, die trotz vollständioer Information von risikofreudigen Individuen in unzureichendem Maße nachgefragt werden. Da zusätzliche Informationen in diesen Fällen keine Präferenzänderungen bewirken, dürfte eine Korrektur der individuellen Verhaltensweisen lediglich durch den Einsatz regulativer meritorischer Instrumente zu erreichen sein. Entweder sind dann - bei Aufrechterhaltung der Marktpreiskoordination - gesetzliche Regelungen zu schaffen (z.B. Anschnallpflicht) oder die Güterbereitstellung müßte öffentlich über ein Angebot bis zur Sättigung mit Benutzungszwang erfolgen ( $z$.B. Schutzimpfung).

Die Diskussion hat gezeigt, daß eine generelle Entscheidung zugunsten der Information oder für eine Meritorisieruna weder aus Effektivitäts- noch aus Effizienzgesichtspunkten gefällt werden kann. Verzerrte Konsumentenpräferenzen haben unterschiedliche Ursachen und diese sind zu berücksichtigen. Konstatiert werden kann lediglich folgendes: Beruht die Präferenzverzerruna auf durch Werbung initiierte Desinformation, so durfte als Gegencewicht zur Werbung eine verbesserte Informationstätigkeit nicht nur effektiver sondern auch effizienter sein als die Meritorisierung. Ist hingegen eine ausgeprägte Risikobereitschaft der Grund für unerwünchtes Nachfrageverhalten der Konsumenten, so stellt offensichtlich eine Meritorisierung bzw. Demeritorisierung die überlegene Interventionsform dar. Darüber hinaus zeiat die Praxis, daß es häufig weniger um eine strenge Alternativentscheidung als vielmehr um ein geeignetes Policy-Mix geht. Schließlich kann aber ein endgültiges Urteil allenfalls dann gefällt werden, wenn die verschiedenen Formen der Meritorisierung ins Kalkül einbezogen werden.

Zu (b): Die Korrektur der Konsumentenpräferenzen durch Meritorisierung ist prinzipiell sowohl mit Hilfe der objektförderung als auch mittels Subjektförderung zu erreichen, ohne daß die Güterbereitstellung über den Marktpreismechanismus aufgegeben werden muß. Bei der reinen objektförderung werden Marktanbietern Subventionszahlungen oder Steuererleichterungen gewährt, die - eine Weiter- 
Gabe vorausgesetzt - Preissenkungen der betreffenden Güter bewirken. Diese Angebotsförderung hat eine indirekte Begünstigung sämtlicher Nachfrager zur Folge, ohne daß spezifische Konstellationen auf der Nachfrageseite Berücksichtigung finden. Dagegen wird bei der Subjektförderung der Konsum bestimmter Güter direkt über gebundene Transferzahlungen an ausgewählte Nachfrageraruppen begüstigt, d.h. es wird bewußt an jene individuellen Merkmale der Nachfrager angeknüpft, die als Indikatoren für vermutete Präferenzverzerrungen gelten (soziale Stellung, Einkommenshöhe).

Für die Objekt- wie für die Subjektförderung gilt gleichermaßen, daß die gewünschte Korrektur der Präferenzen um so eher erreicht wird, je stärker die Konsumenten auf die Preissenkung des betreffenden Gutes mit einer Ausweitung ihrer Marktnachfrace reagieren, je nöher also die Elastizität der Nachfrạe in bezug auf den Marktpreis ausfällt. Leider lassen sich ex ante selten verläßliche Aussagen über die Preiselastizitäten der Nachfrage machen, so daß der erforderliche Umfang der finanziellen Förderung im voraus kaum zu bestimmen ist.

Abgesehen von diesen generellen Schwierigkeiten leidet die reine objektförderung unter dem Nachteil, daß sämtliche Konsumenten, undifferenziert nach dem $A$ usmaß der bei innen vermuteten Präferenzverzerrungen, gleichermaßen begünstigt werden. Besteht die allokative Zielsetzung darin, generelle Versorgungsmängel, wie z. B. die Wohnungsnot in der Nachkriegszeit, zu beseitigen, kann die objektförderung als effektives und effizientes Instrument angesehen werden. Gilt es hingegen, die Wohnverhältnisse ausschließlich jener Gruppen zu verbessern, bei denen verzerrte Präferenzen vermutet werden, ist ein differenzierter Einsatz finanzieller Instrumente erforderlich. Mit der objektförderuna wird dann nicht nur das eigentliche Ziel der Meritorisierung verfehlt, sondern es sind auch neue allokative Verwerfungen zu erwarten.

Effektivitäts - und Effizienzaspekte sprechen deshalb für eine Subjektförderung. Grundsätzlich besteht hier die Möglichkeit, 
Konsumentengruppen differenziert nach dem Ausmaß der vermuteten Präferenzverzerrungen zu begünstigen. Meritorische Eingriffe bewirken dann zumindest Korrekturen in die gewünschte Richtung, und im Vergleich zur objektförderung ist eine Einsparung öffentlicher Finanzmittel zu erwarten. 1) Schwierigkeiten bereitet bei der Subjektförderung allerdings die Identifikation der gemäß dem Meritorisierungsziel zu fördernden Nachfragergruppen. Begünstigt werden heute überwiegend soziale Gruppen wie Schüler, Studenten, Arbeitslose und Rentner oder - wie z.B. beim Wohngeld - Personen bis zu einer bestimmten Einkommenshöhe. Nur wenn die soziale Stellung und/oder die Einkommenshöhe tatsächlich geeionete Indikatoren für die Präferenzverzerrung darstellen, kann das Meritorisierungsziel realisiert werden. Ist dies aber - wie zu vermuten ist - nicht der Fall, so hat die Subjektförderuna ebenfalls effizienzmindernde Mitnahmeeffekte zur Folge.

Eine Reihe von Gütern, die im Zusammenhang mit dem Meritorisierungsargument eine Rolle spielen, werden in der Bundesrepublik nicht über den Markt angeboten sondern öffentlich, d.h. entweder in Form des Angebots bis zur Sättigung (z.B. Schulbildung) oder in Form der Kontingentierung (z.B. Sozialwohnungen), bereitaestellt. Wie bei der finanziellen Förderung im Rahmen der Marktpreiskoordination ist auch hier prinzipiell eine Unterscheidung in objekt- und subjektbezogene Interventionen möglich. Während beim Angebot bis zur Sättigung die unentgeltiche oder im Vergleich zum Marktpreis zumindest erheblich verbilligte Güterbereitstellung für sämtliche Nachfrager in gleichem Maße erfolat, wird bei der Kontingentierung die begünstigte Bereitstelluna auf jene Nachfrager beschränkt, die konkrete individuelle Merkmale aufweisen. ${ }^{2)}$ uberwiegend werden jedoch objekt- und subjekt-

1) Einsparungseffekte dïften selbst dann eintreten, wenn die - den Effizienzvorteil schmälernden - höheren administrativen Kosten der Subjektförderung berücksichtigt werden.

2) Im Hinblick auf einen Effizienzvergleich zwischen den beiden Koordinationsmechanismen Angebot bis zur Sättigung und Kontingentierung haben deshalb die im vorigen Abschnitt aufgeführten Argumente weitgehend Gültiakeit. Im allgemeinen durfte die Kontingentierung das effizientere Verfahren sein. 
bezogene Elemente gekoppelt, d.h. die weitgehend über allaemeine Finanzmittel öffentlich bereitgestellten Güter kommen zunächst allen Nachfragern gleichermaßen zugute, darüber hinaus werden aber zusätzlich gezielt Nachfrageraruppen wie z.B. Schüler, Studenten, Rentner, Arbeitslose etc. beaünstict. Dies gilt u.a. für öffentliche Leistungen wie Theater, Museen oder Bibliotheken.

Zu (c): Die Ausführungen zeigen, daß die Korrektur der Konsumentenpräferenzen grundsätzlich keine Abkehr von der Marktpreissteuerung verlangt, das Meritorisierungsziel vielmehr bereits durch den Einsatz staatlicher Förderunasinstrumente zu Unterstützung der Marktnachfrage erreicht werden kann. Zu prüfen bleibt indes, ob auch im Hinblick auf das Kriterium der allokativen Effizienz eine staatlich gestützte. Marktlösung der öffentlichen Güterbereitstellung vorzuziehen ist.

Am Beispiel des Gutes Theateraufführungen lassen sich einige allokative Implikationen der Alternative "subventioniertes Markttheater" oder "Staats(Stadt)-Theater" skizzieren. " Wir gehen zunächst davon aus, daß das Marktangebot an Theateraufführungen unter den folgenden Bedingungen zu erstellen ist:

1. Den privaten Theaterunternehmen werden nicht kostendeckende Subventionen in fester Höhe gewährt. ${ }^{2}$ )

1) Die gegenwärtige Kosten-Erlös-Struktur eines repräsentativen öffentlichen Theaters ist unter Effizienzgesichtspunkten nicht anders als deprimierend zu bezeichnen. Die Grenzaufführungskosten übertreffen die Grenzaufführunaserlöse etwa um das Fünffache. Eine Deckung der Durchschnittskosten ist selbst durch beliebige Erhöhungen der Aufführungszahlen eines Theaterstuickes (economies of scale) nicht zu erreichen. Im Gegenteil, jede weitere Aufführung eines Stückes steigert die Verluste des öffentlichen Theaters. Vgl. dazu H. Hanusch, Privatisierung einer öffentlichen Tätigkeit: Das Beispiel Theater, in: Staat und Wirtschaft, hrsg. von C.C. von Weizsäcker, SchdVSocpol N.F., Bd. 102, Berl in 1979, S. $171 \mathrm{ff}$, insbesondere S. $177 \mathrm{f}$.

2) Wie die Subventionszahlungen im einzelnen zu gestalten sind, muß hier nicht erörtert werden. Von Bedeutung ist allein, daß die Höhe der Subventionen ex ante limitiert wird, um die negativen allokativen Aliswirkunaen der staatlichen Defizitgarantie zu vermeiden, die insbesondere darin bestehen, daß Unternehmen zu einem Subventionenmaximierungsverhal ten anceregt werden. 
2. Das Meritorisierungsziel soll dann als erreicht gelten, wenn die bisherige Preisdifferenzierung in Form ermäßiater Eintrittspreise für bestimmte Besuchergruppen bestehen bleibt.

3. Das Repertoire und die Qualität der Aufführungen ist aufrecht zu erhalten. Die Bewahrung des traditionellen Bildungs- und Kulturtheaters wird mithin nach wie vor als öffentliche Aufgabe betrachtet. Die Erfüllung dieser Aufgabe durch Markttheater erfordert dann einen regulativen statichen Eingriff in Form von Auflagen für die privaten Anbieter.

Ein Marktangebot wird unter diesen Bedingungen nur dann zustande kommen, falls die privaten Theaterunternehmen gegenüber den öffentlichen Theatern Spielräume für Kostensenkungen und/oder Erlössteigerungen nutzen können, die ihnen eine Gewinnerzielung ermöglichen. In einer Fallstudie über die Möglichkeiten der Privatisierung öffentlicher Theater kommt HANUSCH zu dem Ergebnis, daß eine Kostenentlastuna allenfalls durch den Abbau überhöhter Personalbestände möglich sei, während eine Verbesseruna. der Erlössituation u.a. durch eine Anhebung der Preise (Ausschöpfuna der unausgenutzten Zahlungsbereitschaft der Theaterbesucher) sowie durch eine Erhöhung der Platzkapazität (Rückkehr zum Massentheater antiker Prägung) erreicht werden könne. " ${ }^{1)}$ obschon also Spielräume für eine Verbesserung der Erlös- und Kostensituation vorhanden sind, bleibt fraglich, ob unter den aenannten Bedingungen ein Marktangebot gesamtwirtschaftliche Effizienzvorteile erbringt. Je geringer nämlich die spielräume ausfallen, umso höhere Subventionszahlungen werden nötig. Je höher aber die Subventionszahlungen sind, desto schmaler wird zwangsiäufia der Effizienzvorteil. Darüber hinaus sind zusätzliche Interventionskosten zu berücksichtigen, die bei der Kontrolle der vom Staat gemachten Auflagen entstehen.

1) Vgl. dazu im einzelnen $\mathrm{H}$. Hanusch, Privatisierung einer öffentlichen Tätigkeit: Das Beispiel Theater, a.a.0., S. $171 \mathrm{ff}$. 
Erheblich bessere Chancen der rentablen Bewirtschaftung bieten sich den privaten Theaterunternehmen, falls die Bedinguna, Repertoire und Qualität des Angebots zu erhalten, aufgegeben wird. Es ist zu erwarten, daß die privaten Anbieter dann eine Verbesserung ihrer Erlös-Kosten-Situation anstreben, indem sie beispielsweise das Repertoire verkleinern und die Zahl der Aufführungen je Inszenierung erhöhen oder dazu übergehen, vornehmlich bekannte, mit geringem Personal- und Sachaufwand verbundene Unterhaltungsstuicke zu spielen. 1) Kein Zweifel, das Markttheater besäße unter diesen Umständen einen beträchtlichen Effizienzvorteil gegenüber dem gegenwärtigen öffentlichen Theater. Zudem werden die öffentlichen Haushalte weiter finanziell entlastet, da nun die Interventionskosten zur Kontrolle der staatlichen Auflagen entfielen und infolge der verbesserten Erlös-Kosten-Situation auch die Subventionszahlungen reduziert werden könnten. Freilich wird damit auch bereits der Pferdefuß dieser Lösung offensichtlich. Die Erhaltung des Bildungs- und Kulturtheaters kann jetzt kaum mehr als öffentliche Aufgabe begriffen werden, d.h. die dritte der oben formulierten Bedingungen ist aufzuheben. Zugleich verlieren aber auch die beiden ersten Bedingungen ihren Sinn, denn die Notwendigkeit eines meritorischen Eingriffes entfällt und folglich läßt sich auch eine Subventionszahlung nicht mehr begründen. Weiter: Die bestehende Nachfrage nach Leistungen des Bildungs- und Kulturtheaters kann aufgrund des oberaangs zum Geschäfts- und Boulevardtheater nicht mehr befriedigt werden, d.h. zwangsläufig werden nun die Präferenzen jener Individuen verletzt, die das bisherige Angebot konsumiert haben. Mithin fallen Angebot und Nachfrage erneut auseinander. Und schlieBlich: Die Produkte des Bildungs- und Kulturtheaters besitzen neben der privaten auch öffentliche Nutzungskomponenten wie z.B. die Erhaltung der Kultur- und Theatertradition von einer Generation zur nächsten. 2) Die aus einzelwirtschaftlicher Sicht effizientere

1) Vg1. H. Hanusch,Privatisierung einer öffentlichen Tätigkeit: Das Beispiel Theater, a.a.0., S. $187 \mathrm{ff}$.

2) Vgl. dazu insbesondere C. A. Andreae, Verteilungswirkungen der Theaterfinanzierung, in: Offentliche Finanzwirtschaft und Verteilung IV, hrsg. von W. Dreißig, SchdVSocpol N.F., Bd. 75/IV, Berlin 1976, S. 134. 
marktwirtschaftliche Lösung erfährt demzufolge in der gesamtwirtschaftlichen Betrachtung eine Relativierung, sobald der "kulturpolitischen Verpflichtung" ein besonderes Gewicht beigemessen wird. Diese Gewichtung vorzunehmen, obliegt freilich nicht dem ókonomen, sondern dem Politiker.

Zusammenfassend läßt sich feststellen, daß die unter Effizienzgesichtspunkten zu empfehlende Interventionsalternative zur Korrektur verzerrter Konsumentenpräferenzen entscheidend davon abhängt, auf welche konkreten Ursachen die Präferenzverzerrungen zurückzuführen sind, zudem aber auch davon beeinflußt wird, welche Bedingungen am jeweiligen Markt herrschen und welche spezifischen Charakteristika dem betreffenden Gut zugewiesen werden:

- Sind die Präferenzverzerrungen - wie im Falle überbordender Werbung - Folge irreführender Informationen, so ist als Geqenstrategie eine gezielte öffentliche Informationstätigkeit effizienter als eine Meritorisierung sämtlicher "werbungsgeschädigter" Guiter.

- Sind die verzerrten Konsumentenpräferenzen primär Ausdruck einer unerwijnscht hohen individuellen Risikobereitschaft, dann ist von einer zusätzlichen Informationsbereitstellung keine Präferenzänderung zu erwarten, vielmehr sind in diesem Fall regulative meritorische Interventionen in den Marktpreismechanismus erforderlich.

- Sind die verzerrten Präferenzen aufarund unvollständiger Informationen entstanden, so ist zu ihrer Korrektur im Regelfall ebenfalls keine Abkehr von der Marktpreissteuerung notwendig. Abgesehen vom Fall genereller Versorgunasmängel am Markt wird hier überwiegend der Einsatz subjektbezogener finanzieller Instrumente als die effiziente Interventionsform anzusehen sein. Eine Bereitstellung über nichtmarktliche Koordinationsmechanismen ist freilich dann die zu empfehlende Alternative, falls bereits die Verfolgung des Meritorisierungszieles und/oder ausaeprägte öffentliche Nutzungskomponenten ein effizientes Marktangebot unmöglich machen. 


\section{Ergebnisse}

Die kritische Würdigung der ökonomischen Beurteilunaskriterien fur ein allokatives Marktversagen hat gezeigt, daß mit der Feststellung eines Interventionsbedarfes noch nicht notwendigerweise die Ablösung der Marktpreissteuerung und der übergang zu nichtmarktlichen Koordinationsmechanismen erforderlich wird. Anhand der ökonomischen Entscheidungskriterien wird vielmehr deutlich, daB als alternative Strategie zur Erreichung einer möalichst effizienten Allokation Eingriffe in den Marktpreismechanismus auf der Interventionsebene der Instrumente oder/und der Institutionen zu empfehlen sind.

Inwieweit ein beobachtbares monopolistisches Unternehmensverhal ten tatsächlich einen Interventionsbedarf begründet, hängt einerseits von der Höhe der durch die Monopolbildung verursachten sozialen Kosten und andererseits von der Höhe der mit dem Einsatz verschiedener Interventionsformen zur Reduzierung der Wettbewerbsbeschränkungen verbundenen Interventions - und Transaktionskosten ab. Die dem Staat zur Verfügung stehenden Instrumente, die institutionellen Gestaltungsmöglichkeiten und die prinzipiell denkbaren Koordinationsmechanismen sind so zu wählen, daß die durch Monopolisierung bedingte Gesamtkostenbelastuna (Effizienzverluste) für die Volkswirtschaft ein Minimum wird.

Im Falle sinkender Durchschnittskosten besteht die unter ökonomischen Aspekten adäquate Strategie darin, Monopolstellungen zuzulassen und somit das Marktpreissystem weitgehend außer Kraft zu setzen. Die Aufrechterhaltung eines natürlichen Monopols erzeugt im Gegensatz zu den künstichen Monopolen damit stets einen Interventionsbedarf seitens des States. Mit der staatiichen Regulierung und der Beteiliguna des States an privaten Monopolen bieten sich zwei alternative Formen öffentlicher Aufabenwahrnehmung an, wobei die staatliche Requlierung als die überlegene Interventionsform anzusehen ist.

Aus der Unfähigkeit des Marktpreissystems, zukünftige Knappheitsverhältnisse zu signalisieren, ergeben sich differenzierte Inter- 
ventionsempfehlungen. Im Hinblick auf angewandte Forschung und Entwicklung düften im privaten Sektor immer dann ausreichende Innovationsreize induziert werden, wenn der öffentliche Sektor auf der Instrumentenebene unterstuitzend in den Marktmechanismus eingreift (Erhöhung der Ertragssicherheit der Unternehmen bzw. Senkung der betrieblichen Investitionskosten). In bezug auf risikoreiche Großprojekte sind hingegen temporäre Eingriffe auf der Institutionenebene günstiger zu beurteilen (z.B. öffentliche Unternehmen als "Markterkunder"). Die Grundlagenforschunq muß schließlich infolge der Unmöglichkeit, Wirtschaftssubjekte von den Forschungsergebnissen auszuschließen, vom Staat getragen werden (Interventionen auf der Ebene der Koordinationsmechanismen und der Institutionen).

Die Erörterung externer Effekte der Produktion hat gezeigt, daß der marktwirtschaftliche Königsweg, die Internalisierung externer Nachteile mittels Fusion oder Verhandlung, nur in Ausnahmefällen zu allokativen Verbesserungen führt. Das bedeutet, daß mit der Identifikation relevanter externer Effekte in der Regel staatliche Interventionen notwendig werden. Ahnlich wie die Ausweitung des Marktpreissystems ist freilich auch die Ablösung der Marktpreissteuerung durch nichtmarktliche Koordinationsmechanismen (z.B. Kontingentierung) kaum als ein geeigneter Ansatz zur Lösung des Externalitätenproblems zu werten. Jenseits dieser beiden Extreme steht jedoch ein breiter Fächer alternativer staatlicher Instrumente zur Verfüquna, deren relative Vorteilhaftigkeit allerdings am Einzelfall zu prifen ist.

Gilt als empirisch gesichert, daß bei öffentlichen Gütern der Ausschluß von Konsumenten nur in den seltensten Fällen technisch völlig unmöglich ist, so rücken die Kosten des Ausschlusses in den Blickpunkt des Interesses. Die Entscheidungsfrage lautet dann: Unter welchen Bedingungen ist das Anaebot von Gutern und Diensten über einen Koordinationsmechanismus, dem das AusschluBprinzip zugrundeliegt, gesamtwirtschaftlich effizienter als die Guiterbereitstellung über einen Mechanismus ohne Anwendung des Ausschlußprinzips? Eine eindeutige Antwort ist nur möalich, falls 
die Güter eine ausgeprägte Nichtrivalität im Konsum aufweisen. Die öffentliche Bereitstellung über ein Angebot bis zur Sättigung ist hier der gesamtwirtschaftlich effiziente Koordinationsmechanismus, es sei denn, ein einzelnes Individuum oder eine Gruppe ziehen aus dem Konsum eines Gutes einen Nutzen, der die Bereitstellungskosten dieses Gutes übertrifft (z.B. privates Feuerwerk). Darüber hinaus jedoch wird die Wahl des effizienten Koordinationsverfahrens entscheidend von der Höhe der Transaktionskosten bestimmt.

Welche Interventionsalternative im Zusammenhana mit dem Problemkreis meritorischer Güter unter Effizienzgesichtspunkten zu empfehlen ist, hängt ganz wesentlich davon ab, auf welche konkreten Ursachen die Präferenzverzerrungen der Konsumenten zurückzuführen sind. Sind sie - wie im Falle überbordender Werbuna - Folae irreführender Informationen, so ist als Gegenstrateaie eine aezielte öffentliche Informationstätigkeit effizienter als eine Meritorisierung sämtlicher "werbungsgeschädigter" Guiter. Sind die verzerrten Konsumentenpräferenzen primär Ausdruck einer unerwünscht hohen individuellen Risikobereitschaft, dann ist von einer zusätzlichen Informationsbereitstelluna keine Präferenzänderung zu erwarten, vielmehr sind in diesem Fall regulative Interventionen in den Marktpreismechanismus erforderlich. Sind die verzerrten Präferenzen aufgrund unvollständiger Informationen entstanden, so ist zu ihrer Korrektur im Regelfall ebenfalls keine Abkehr von der Marktpreissteuerung notwendig, vielmehr wird der Einsatz subjektbezogener finanzieller Instrumente als die effiziente Interventionsform anzusehen sein. Eine Güterbereitstellung über nichtmarktliche Koordinationsmechanismen ist freilich dann die zu empfehlende Alternative, falls ausgeprägte öffentliche Nutzungskomponenten ein effizientes Marktangebot unmöqlich machen. 


\section{ZUSAMMENFASSUNG}

1. Die Wissenschaft hat - trotz umfangreicher Beschäftiouna mit dem Problemkreis - bisher wenig zur allaemeinen Begriffsbestimmung öffentlicher Aufgaben beigetragen. Der häufig ohne Erläuterung verwendete Terminus "öffentliche Aufgaben" bildet vielmehr die klammer für die in rechts-, verwaltunas-, politik- und wirtschaftswissenschaftlichen Untersuchungen üblichen Termini wie Staatsaufgaben, Verwaltunasaufaaben, öffentliche und meritorische Güter, Kollektivgüter, öffentliche Leistungen oder öffentliche Bedürnisse. In der terminoloaischen Variationsbreite spiegelt sich vor allem das unterschiedliche Erkenntnisinteresse der beteiligten Wissenschaften wider. Darüber hinaus besitzt der Begriff der Aufgabe selbst eine große Spannweite, d.h. ihr materieller Gehalt kann recht unterschiedliche Dimensionen aufweisen.

2. Soll aus wirtschaftswissenschaftlicher Sicht ein Beitrag zur Abgrenzung öffentlicher von privaten Aufaaben geleistet und sollen Kriterien für eine ökonomisch zweckmäßige Aufgabenverteilung in der Marktwirtschaft entwickelt werden, so lassen sich die methodischen Ansätze auf folgende Fragestellunaen verengen:

- Mit Hilfe welcher Kriterien können die gegenwärtia in der Bundesrepublik Deutschland als öffentliche Aufgaben betrachteten Aktivitäten von den privaten Aufqaben abgegrenzt werden (deskriptiver Ansatz)?

- Welche Bestimmungsgründe können zur Erkläruna des geaenwärtigen Bestandes und der zukünftigen Entwickluna öffentlicher Aufgaben herangezogen werden (positiver Ansatz)?

- Welche Kriterien sind für die Ableitung einer ökonomisch zweckmäßigen Aufgabenverteilung in der Marktwirtschaft maßgebend (normativer Ansatz)?

Gegenstand dieser Untersuchung sind die deskriptiven und normativen Kriterien. 
3. Der Versuch einer deskriptiven Aufgabenabarenzuna mittels eines einzigen umfassenden Kriteriums muß an der Komplexität öffentlicher Aufgaben scheitern. Im Rahmen einer systematisch angelegten Deskription wird deshalb die Tauglichkeit verschiedener Kriterien einer Aufgabenabgrenzung, die unterschiedlichen Fragestellungen Rechnung tragen, überprüft. Einzelne Kriterien können dabei zu Kriterienkategorien zusammengefaßt werden, und zwar zu

- funktionalen Kriterien, welche die Zuordnung anhand der speziell öffentlichen oder privaten Zwecksetzuna einer Aufgabe vornehmen;

- institutionellen Kriterien, welche die "offentlichkeit" oder "Privatheit" einer Aufgabe allein von der Zugehörigkeit des Aufgabenträgers zum öffentlichen bzw. privaten Sektor abhängig machen;

- technisch-ökonomischen kriterien, welche entweder aus den spezifischen Eigenschaften einer Aufgabe auf ihre "offentlichkeit" schließen oder die Ausgestaltung des Koordinationsmechanismus, über den die Aufgabenerfülluna erfolat, zum entscheidenden Merkmal erheben.

4. Der Erkenntniswert einer Beschreibung öffentlicher und privater Aufgaben mittels funktionaler Kriterien muß als gering veranschlagt werden. Versuche, öffentliche Funktionen zu einem öffentlichen Kernbereich zusammenzufassen oder öffentliche Aufgaben anhand funktionaler Gesichtspunkte systematisch zu ordnen, tragen genauso wenig zur Identifikation öffentlicher und privater Aufgaben bei wie der Versuch, eine Trennuna von Aktivitäten in solche, die dem Gemeinwohl dienen, und solche, die aus dem Eigennutzmotiv hervorgehen, durchzuführen.

Eine Erörterung der institutionellen Abgrenzungskriterien zeigt, daß es zwar gelingt, anhand der Rechtsformen und der Eigentumsverhältnisse öffentliche und private Aufaabenträger zu unterscheiden. Sie macht aber zugleich sichtbar, daß öffentliche Aufgaben sowohl von öffentlichen als auch von pri- 
vaten Trägern durchgeführt und private Aufgaben nicht nur von privaten, sondern auch von öffentlichen Trägern wahrgenommen werden. Zudem verdeutlicht sie die Kompetenzstruktur der Aufgabenträger und gibt damit einen Oberblick über den Fächer alternativer staticher Instrumente.

Eine differenzierte Bewertung ist bezüglich der technisch-ökonomischen Abgrenzungskriterien erforderlich. Das Konzept der öffentlichen Güter leistet keinen nennenswerten Beitrag zur Aufgabenabgrenzung, da die Unterscheidung öffentlicher und privater Güter mit Hilfe gutstechnischer Eigenschaften nur mit erheblichen Einschränkungen gelingt. Es können allenfalls einige polare Fälle erfaßt werden, während die Zahl der nicht eindeutig zurechenbaren Mischformen bei weitem uiberwiegt. Das Konzept der Koordinationsmechanismen besitzt dagegen den Vorteil, daß die Güter und Dienste den einzelnen Mechanismen nicht nur überwiegend zugeordnet werden können, sondern auch unabhängig von der Trägerschaft die weitgehende Erfassung marktlicher und nichtmarktlicher Formen der Güterbereitstellung gelingt.

5. Insgesamt liefern die deskriptiven Abgrenzungsversuche eine eindrucksvolle Dokumentation der Komplexität öffentlicher Aufgaben, können zur terminologischen Klarstellung und zur systematisierung der oftmals mit uneinheitlichen Begriffen und aus unklaren Begründungszusammenhängen geführten Privatisierungsdiskussion beitragen und ermöglichen die Vorstrukturierung der für die normative Analyse bedeutsamen Fragestellungen.

6. In der ökonomischen Theorie bildet überwiegend das wohlfahrtsökonomische Modell des allgemeinen Konkurrenzgleichgewichts den Ausgangspunkt für Oberlegungen über die unter Allokationsaspekten "richtige" Aufgabenverteilung in der Marktwirtschaft. Der Versuch, mit Hilfe des wohlfahrtsökonomischen Modells Bestlösungen abzuleiten, somit Abweichungen von einem Optimum feststellen zu können, und auf diesem Wege dem öffentlichen Sektor klar umrissene Aufgaben zuzuweisen, schlägt jedoch aus mehreren Gründen fehl: 
- Das Pareto-Kriterium formuliert Effizienzbedingunaen für ein inhaltsleeres Zielsystem und läßt zudem nur in einem äuBerst engen Rahmen wirtschaftliche Reorganisationen $z u$.

- Die Realisierung der wohlfahrtsökonomischen Marginalbedingungen gewährleistet lediglich ein lokales optimum und führt damit zu einer rein statischen Betrachtunasweise.

- Es wird vernachlässigt, daß sämtliche wirtschaftlichen Reorganisationen kosten verursachen und überdies stets nur neue suboptimalitäten hervorbringen können.

7. Zur Wahl steht nicht ein mit Mängeln behafteter oder ein ideal funktionierender Koordinationsmechanismus ("Nirwana-Ansatz"), sondern die einzig akzeptable Vorgehensweise - jedenfalls dann, wenn die Wissenschaft Entscheidungshilfe für die Politik bieten will - besteht darin, realisierbare Alternativen zu vergleichen. Die Leitlinien zur Beantwortung der Frage nach der ökonomisch zweckmäßigen Aufgabenverteilung in der Marktwirtschaft sind deshalb in mehreren Schritten zu erarbeiten.

8. In einem ersten Schritt werden die ökonomischen Kriterien entwicke1t, die Funktionsstörungen des Marktpreissystems belegen. Dabei werden Marktmängel auf der Angebotsseite des Wirtschaftsprozesses (monopolistisches Unternehmensverhalten, sinkende Durchschnittskosten, fehlender Ausweis zukünftiger Knappheitsverhältnisse, externe Effekte der Produktion) und Marktmängel auf der Nachfrageseite des Wirtschaftsprozesses (öffentliche Güter, meritorische Güter) unterschieden. Anders als den in der wohlfahrtsökonomischen Literatur abgeleiteten "Fällen des Marktversagens" wird ihnen allerdings nicht von vornherein implizit die Rolle von Entscheidungskriterien für eine Aufgabenverteilung zugewiesen, sondern, da sie zunächst nur die Legitimationsgrundlage für öffentliche Interventionen bilden, sind sie als Beurteilungskriterien und damit als notwendige Bedingungen für einen Eingriff in den Marktpreismechanismus und gegebenenfalls für eine Ablösung des Marktpreissystems zu betrachten. 
9. Wird anhand der Beurteilungskriterien ein Interventionsbedarf konstatiert, so ist in einem zweiten Schritt eine genaue Analyse der prinzipiell denkbaren Interventionsebenen und -formen vorzunehmen. Zu untersuchen ist, ob

- der notwendige Eingriff in die Marktpreiskoordination durch den Einsatz regulativer, finanzieller oder informatischer Instrumente des States erfolgen sollte;

- die Güterversorgung weiterhin privaten Institutionen überlassen bleiben oder aber öffentlichen Trägern überantwortet werden sollte;

- der Obergang zu einem nichtmarktlichen Koordinationsmechanismus (Kontingentierung, Angebot bis zur Sättigung, Windhundverfahren oder Verlosung) als Problemlösungsstrategie zu empfehlen ist.

10. Die Auswahl geeigneter Interventionsformen hat schlieBlich in einem dritten Schritt auf Grundlage ökonomischer Entscheidungskriterien zu erfolgen, die dann den Charakter hinreichender Bedingungen für die Korrektur der Marktpreissteuerung besitzen. Von besonderer Bedeutung sind in diesem Zusammenhang Effizienzvergleiche, die aber auf eine möglichst breite Basis gestellt werden müssen. Zu berücksichtigen sind nicht nur die direkten und indirekten kosten, die mit den Interventionen in das Marktpreissystem verbunden sind, sondern auch die zur Betreibung eines Koordinationsmechanismus anfallenden Transaktionskosten. Darüber hinaus sind soziale Kosten zu beachten, die zwar gesamtwirtschaftlich existent sind, aber keine Berücksichtigung im Rechensystem eines Koordinationsmechanismus finden. 
Albert Peters - 978-3-631-75163-3

Downloaded from PubFactory at 01/11/2019 07:34:44AM

via free access 


\section{LITERATURVERZEICHNIS}

ALBERT, Hans: Das Ende der Wohlfahrtsökonomik, in: Gewerkschaftliche Monatshefte, 1958, S. 33-36.

-: Wertfreiheit als methodisches Prinzip, in: Probleme der normativen okonomik und der wirtschaftlichen Beratung, hrsg. von E. von Beckerath und H. Giersch, Schriften des Vereins für Socialpolitik, N. F., Bd. 29, Berlin 1962, S. 32-63.

ANDEL, Norbert: Zur Diskussion über Musgraves Begriff der "merit wants", in: Finanzarchiv, N.F., Bd. 28 (1969), S. 209-213.

ANDREAE, Clemens-August: Verteilungswirkungen der Theaterfinanzierung, in: Offentliche Finanzwirtschaft und Verteilung IV, hrsg. von W. Dreißia, Schriften des Vereins für Socialpolitik, N.F., Bd. 75/IV, Berlin 1976, S. $131-150$.

v. ARNIM, Hans Herbert: Gemeinwohl und Gruppeninteressen, Frankfurt/M. 1977.

ARROW, Kenneth J.: Social Choice and Individual Values, 2. Auf1., New York 1963.

-: The Organization of Economic Activity: Issues Pertinent to the Choice of Market versus Nonmarket Allocation, in: The Analys is and Evaluation of Public Expenditures: The PPB System, A Compendium of Papers submitted to the Subcommittee on Economic in Government of the Joint Economic Committee Congress of the United States, Vol. 1, Washington 1969, S. 47-64.

ARROW, Kenneth J./DEBREU, Gërard: Existence of an Equilibrium for a Competitive Economy, in: Econometrica, 22. Jg. (1954), S. 265-290.

AUBEL, Peter van: Die gemischtwirtschaftliche Unternehmung, in: Handbuch der Kommunalen Wissenschaft und Praxis, 3. Bd., Berlin-Göttingen-Heidelberg 1959 , S. $877-892$.

AULD, D. A. L./BING, P. C.: Merit Wants: A Further Analysis. in: Finanzarchiv, N.F., Bd. $30(1971 / 72)$, S. 257-265.

BADELT, Christoph: Sozioökonomie der Selbstorganisation, Frankfurt/M., New York 1980.

-: Selbstorganisation kommunaler Aufgaben, in: Das öffentliche Haushaltswesen in Osterreich, 21. Jg. (1980), S. 57-69.

BARTELS, Hildegard: Das Einkommenskonto für den Staat in den Volkswirtschaftlichen Gesamtrechnungen, in: Wirtschaft und Statistik, 1955, S. 119-131.

-: Das Kontensystem für die Volkswirtschaftlichen Gesamtrechnungen der Bundesrepublik Deutschland, in: Wirtschaft und Statistik, 1960, S. 317-344. 
BARTLING, Hartwig: Leitbilder der Wettbewerbspolitik, München 1980.

BATOR, Francis M.: The Anatomy of Market Failure, in: Quarterly Journal of Economics, Vol. 72 (1958), S. 351-379.

BAUER, Rudolph: Wohlfahrtsverbände in der Bundesrepublik. Materialien und Analysen zur Organisation, Programmatik und Praxis, Weinheim und Basel 1978.

BAUM, Gerhart: Probleme der Privatisierung öffentlicher Aufgaben, in: Privatisierung - Gewinn für wen?, Vorträge und Diskussionsbeiträge der 21. beamtenpolitischen Arbeitstagung des Deutschen Beamtenbundes, Bonn 1980 , S. 13-38.

BAUMOL, William J./BRADFORD, David F.: Optimal Departures from Marginal Cost Pricing, in: The American Economic Review, Vol. 60 (1970), S. 265274.

BECKER, Erich: Verwaltungsaufgaben, in: Verwaltung, hrsg. von F. Morstein Marx, Berlin 1965, S. 187-214.

BERG, Wilfried: Die Verwaltung des Mangels. Verfassungsrechtliche Determinanten für Zuteilungskriterien bei knappen Ressourcen, in: Der Staat, 15. Jg. (1976), S. 1-30.

BERICHT der Bundesregierung über die Lage der freien Berufe in der Bundesrepublik Deutschland, Bundestagsdrucksache 8/3139 vom 29.8.1979.

BEYME, Klaus von: Interessengruppen in der Demokratie, 4. Auf $1 .$, München 1974.

BISCHOFF, Detlef/NIKUSCH, Karl-0tto: Privatisierung öffentlicher Aufgaben, Berlin, New York 1977.

BLAAS, Wolfgang: Zur Theorie der Mischguiter, in: Der öffentliche Sektor, Bd. 4 (1978), S. 63-90.

BLANKART, Charles B.: Die wirtschaftspolitische Bedeutung von Scalenerträgen öffentlicher Unternehmen, in: Zeitschrift für öffentliche und gemeinwirtschaftliche Unternehmen, 2. Jg. (1979), S. 1-25.

BLAUM, Kurt: Rotes Kreuz, in: Handwörterbuch der Sozialwissenschaften, 9. Bd., Göttingen 1956 , S. 43-44.

BOBEL, Ingo: Wohlfahrtsverluste durch Marktmacht: Eine Untersuchung für den Bereich der Industrie der Bundesrepublik Deutschland, in: Jahrbücher für Nationalökonomie und Statistik, 197. Jg. (1982), S. 509-520.

BODECKER, Wilhelm: Allokations- und Distributionsprobleme bei Kollektivguitern, Meisenheim am Glan 1972.

BOHRET, Carl: Entscheidungshilfen für die Regierung, Opladen 1970.

BOHRET, Carl/JANN, Werner/JUNKERS, Marie Therese/KRONENWETT, Eva: Innenpolitik und politische Theorie, Opladen 1979. 
BOS, Dieter: Offentliche Unternehmen, in: Handbuch der Finanzwissenschaft, Bd. 2, 3. Auf7., Tübingen 1980.

BOSSMANN, Eva: Volkswirtschaftliche Probleme der Transaktionskosten, in: Zeitschrift für die gesamte Staatswissenschaft, 138. Bd. (1982), S. 664-679.

BOMBACH, Gottfried: Die öffentliche Finanzwirtschaft im Wirtschaftskreislauf, in: Handbuch der Finanzwissenschaft, Bd. 1, 3. Aufl., Tübingen 1978, S. 53-75.

BONUS, Holger: Offentliche Güter und der Offentlichkeitsarad von Gütern, in: Zeitschrift für die gesamte Staatswissenschaft, Bd. 136 (1980), S. 50-81.

BOULDING, Kenneth E.: Welfare Economics, in: A Survey of Contemporary Economics, Vol. II, hrsg. von F. Haley, Homewood, IIl. 1952, S. 1-34.

-: Economics as a Moral Science, in: American Economic Review, Vol. 59 (1969), S. 1-12.

BOWEN, H.: The Interpretation of Voting in the Allocation of Ressources, in: Quarterly Journal of Economics, Bd. 58 (1943), S. 27-48.

BRANDT, U1rich: Wettbewerbspolitische Ausnahmebereiche. Probleme ihrer Ident ifizieruna und Gestaltung, Frankfurt/M. U.a. 1977.

BRAULKE, Michael: Merit Goods: Einige zusätzliche Anmerkunqen, in: Finanzarchiv, N.F., Bd. $31(1972 / 73)$, S. 307-309.

BREITLING, Ruppert: Die Verbände in der Bundesrepublik. Ihre Arten und ihre politische Wirkungsweise, Meisenheim am Glan 1955.

BRINKMANN, Gerhard: Der Funktionenplan. Anmerkungen eines Verwunderten, in: Der öffentliche Haushalt, Jg. 13 (1972), S. 77-84.

BRUCHE, Gert: Elektrizitätsversorgung und Staatsfunktion. Das Regulierungssystem der öffentlichen Elektrizitätsversorgung in der Bundesrepublik Deutschland, Frankfurt/M. und New York 1977.

BROMMERHOFF, Dieter: Das "Planning-Programming-Budgetina System" (PPBS), in: Finanzarchiv, N.F., Bd. 29 (1970), S. 64-74.

BUCHANAN, James M.: The Theory of Public Finance, in: Southern Economic Journal, Vol. 26 (1960), S. 234-238.

-: What Kind of Redistribution Do We Want?, in: Economica, Vol. 25 (1968), S. $185-190$.

-: The Demand and Supply of Public Goods, 2. Auf1., Chicago 1969.

BUCHANAN, James M./STUBBLEBINE, W. Craig: Externality, in: Economica, N.S., Vol. 29. (1962), S. 371-384. 
BULL, Hans Peter: Die Staatsaufgaben nach dem Grundgesetz, 2. Auf1., Kronberg/Ts. 1977.

BUND DER STEUERZAHLER (Hrsg.): Privatisierung öffentlicher Dienstleistungen, Wiesbaden 1978.

BUNDESMINISTER FOR FORSCHUNG UND TECHNOLOGIE (Hrsq.): Bundesbericht Forschung VI, Bonn 1979.

BUNDESMINISTERIUM DER FINANZEN (Hrsg.): Bericht der Bundesregierung über die Entwicklung der Finanzhilfen und Steuervergünstigungen für die Jahre 1979 bis 1982 gemäß $\S 12$ des Gesetzes zur Förderung der Stabilität und des Wachstums der Wirtschaft vom 8. Juni 1967 (Achter Subventionsbericht), Bonn 1981.

-: Verwaltungsvorschriften zur Haushaltssystematik des Bundes, Funktionenplan, 0.J., 0.0 .

BUSCHOR, Ernst: Theorie der Allokation der Staatsausgaben. Darstellung und kritische Würdigung der Theorie der Staatsausgaben unter dem Aspekt der Allokationsproblematik, Diss., Heersbrugg 1970.

BUTTLER, Friedrich: Explikative und normative Theorie der meritorischen Giuter - eine Problemanalyse -, in: Zeitschrift für Wirtschafts - und Sozialwissenschaften, Bd. 93 (1973), S. 129-146.

CANSIER, Dieter: Marktversagen und staatliche Güterbereitstellung als Merkmale öffentlicher Güter, in: Public Finance, Bd. 27 (1972), S. 421-437.

CHRISTENSEN, Laurits R./GREENE, William H.: Economics of Scale in U.S. Electric Power Generation, in: Journal of Political Economy, Vol. 84 (1976), S. 655-676.

CLARK, John M.: Competition as a Dynamic Process, Washinaton, D.C. 1961.

COASE, Ronald H.: The Problem of Social Cost, in: Journal of Law and Economics, 3. Jg. (1960), S. 1-44.

-: The Lighthouse in Economics, in: Journal of Law and Economics, 17. Ja. $(1974)$, S. 357-376.

COLM, Gerhard: Comments on Samuelson's Theory of Public Finance, in: Review of Economics and Statistics, Bd. 38 (1956), S. 408-412.

-: In Defense of the Public Interest, in: Social Research, 27. Ja. (1960), S. 295-307.

-: Art. Nationalbudget, in: Handwörterbuch der Sozialwissenschaften (HdSW!), Bd. 7, Stuttgart u.a. 1961, S. 535-540.

-: National Goals Analysis and Marainal Utility Economics, in: Finanzarchiv, N.F., Bd. 24 (1965), S. 209-224.

COWLING, Keith/MUELLER, Dennis C.: The Social Costs of Monopoly Power, in: The Economic Journal, 88. Ja. (1978), S. 727-747. 
COX, Helmut/HOBENER, Harald: Wettbewerb. Eine Einführung in die Wettbewerbstheorie und Wettbewerbspolitik, in: Handbuch des Wettbewerbs, hrsg. von H. Cox, U. Jens und K. Markert, München 1981, S. 1-48.

DAHLMANN, Carl J.: The Problem of Externality, in: The Journal of Law and Economics, 22. Jg. (1979), S. 141-161.

DEMSETZ, Harold: Information and Efficiency: Another Viewpoint, in: Journal of Law and Economics, 11. Jg. (1969), S. 1-22.

DENEKE, J. F. Volrad: Die freien Berufe, Stuttgart 1956.

_-: Klassifizierung der Freien Berufe, Köln, Berlin 1969.

DESERPA, Allan C.: Congestion, Pollution, and Impure Public Goods, in: Public Finance, Bd. 33 (1978), S. 68-83.

DEUTSCHER STADTETAG: Privatisierung öffentlicher Aufgaben, DST-Beiträge zur Kommunalpolitik, Heft 2, Köln 1976.

DIECKMANN, Rudolf: Aufgabenkritik in einer Großstadtverwaltung, Schriftenreihe der Hochschule Speyer, Bd. 65, Berlin 1977.

DOWNS, Anthony: Okonomische Theorie der Demokratie, Tübingen 1968.

EASTON, David: A Framework for Political Analysis, Englewood Cliffs 1965.

-: A Systems Analysis of Political Life, New York, London, Sydney 1965.

EICHHORN, Peter: Zum Begriff der gemischtwirtschaftlichen Unternehmung, in: Betriebswirtschaftliche Forschung und Praxis, 18. Jg. (1966), S. 609-619.

_-: Struktur und Systematik kommunaler Betriebe, Stuttgart 1969.

ELLWEIN, Thomas: Einführung in die Regierungs- und Verwaltungslehre, Stuttgart, Berlin, Köln, Mainz 1966.

-: Regierung und Verwaltung, 1. Tei1: Regierung a1s politische Führung, Stuttgart, Berlin, Köln, Mainz 1970.

-: Das Regierungssystem in der Bundesrepublik Deutschland, 4. Aufl., Opladen 1977.

EMMERICH, Volker: Das Wirtschaftsrecht der öffentlichen Unternehmen, Bad Homburg v. d. H. 1969.

ENDRES, Alfred: Die Coase-Kontroverse, in: Zeitschrift für die gesamte Staatswissenschaft, Bd. 133 (1977), S. 637-651.

-: Neuere Entwicklungen in der Theorie der öffentlichen Güter, in: Zeitschrift für die gesamte Staatswissenschaft, Bd. 137 (1981), S. 134-149. 
ENGELS, Wolfram: Privater Wohlstand - Offentliche Armut?, in: Der Staatssektor in der sozialen Marktwirtschaft, hrsg. von D. Duwendag, Schriftenreihe der Hochschule Speyer, Bd. 59, Berlin 1976, S. 149-159.

ESCHENBURG, Theodor: Herrschaft der Verbände?, Stuttgart 1956.

EUCKEN, Walter: Grundsätze der Wirtschaftspolitik, 5. Auf1., Tübingen 1975.

EYNERN, Gert von: Das öffentlich gebundene Unternehmen, in: Archiv für öffentliche und freigemeinwirtschaftliche Unternehmen, Bd. 4 (1958), S. 1-59.

FOLKERS, Cay: Meritorische Güter als Problem der normativen Theorie öffentlicher Ausgaben, in: Jahrbuch für Sozialwissenschaft, Bd. 25 (1974), S. 1-29.

FORSTHOFF, Ernst: Die öffentliche Körperschaft im Bundesstaat, Beiträge zum öffentlichen Recht der Gegenwart, Bd. 3, Tübingen 1931.

FORTE, Francesco: Should "Public Goods" be Public?, in: Papers on Non-Market Decision Making, Bd. 3 (1967), S. 39-46.

FREY, Bruno S.: Moderne politische Okonomie. Die Beziehungen zwischen Wirtschaft und Politik, München und Zürich 1977.

FREY, René L.: Infrastruktur. Grundlagen der Planung öffentlicher Investitionen, 2. Auf1., Tübingen 1972.

-: Theorie und Messung der finanzwirtschaftlichen Umverteilung, in: Neue Aspekte der Verteilungstheorie, hrsg. von G. Bombach, B. S. Frey und B. Gahlen, Tübingen 1974, S. 401-434.

FRIEDRICH, Carl J. (Hrsg.): The Public Interest, Nomos Bd. 5, New York 1962.

GEWERKSCHAFT OTV: Zur Privatisierung öffentlicher Dienstleistungen, Stellungnahme des geschäftsführenden Hauptvorstandes der Gewerkschaft oTV, Heft 1 , Stuttgart 1977.

GEYER, Herbert: Offentliche Güter, in: Handwörterbuch der Wirtschaftswissenschaft, Bd. 5, Stuttgart u.a. 1980, S. 419-431.

GIERSCH, Herbert: Allgemeine Wirtschaftspolitik, Erster Band: Grundlagen, Wiesbaden 1961.

GRONER, Helmut: Die Ordnung der deutschen Elektrizitätswirtschaft, BadenBaden 1975.

-: Wettbewerbspolitische Ausnahmebereiche im GWB: Das Beispiel der Elektrizitätsversorgung, in: Handbuch des Wettbewerbs, hrsg. von H. Cox u.a., München 1981, S. 421-455.

GROSSEKETTLER, Heinz: Konzepte zur Beurteilung der Effizienz von Koordinationsmethoden, in: Jahrbuch für neue Politische Okonomie, 1. Bd. (1982), S. 213-257. 
HÄBERLE, Peter: Offentliches Interesse als juristisches Problem. Eine Analyse von Gesetzgebung und Rechtsprechung, Bad Homburg v.d.H. 1970.

HÄUSER, Karl: Über Ansätze zur Theorie der Staatsausgaben, in: Herbert Timm, Heinz Haller (Hrsg.): Beiträge zur Theorie der öffentlichen Ausgaben, Schriften des Vereins für Socialpolitik, N.F., Bd. 47, Berlin 1967, S. 36-65.

HAMER, Günter: Revidierte Konten der Volkswirtschaftlichen Gesamtrechnungen, in: Wirtschaft und Statistik, 1970, S. 281-288.

HAMM, Walter: Kollektiveigentum. Die Rolle öffentlicher Unternehmen in der Marktwirtschaft, Heidelberg 1961.

HANSMEYER, Karl-Heinrich/RÜRUP, Bert: Staatswirtschaftliche Planungsinstrumente, 2. Auf1., Tübingen, Düsseldorf 1975.

HANSMEYER, Karl-Heinrich: Die Abwasserabgabe als Versuch einer Anwendung des Verursacherprinzips, in: Ökonomische Probleme der Umweltschutzpoltiik, hrsg. von 0. Issing, Schriften des Vereins für Socialpolitik, N.F., Bd. 91, Berlin 1976, S. 65-97.

HANUSCH, Horst: Theorie des öffentlichen Gutes, Göttingen 1972.

-: Reform öffentlicher Leistungen. Beiträge zur Entstaatlichung, BadenBaden 1978.

-: Privatisierung einer öffentlichen Tätigkeit: Das Beispiel Theater, in: Staat und Wirtschaft, Schriften des Vereins für Socialpolitik, N.F., Bd. 102, Berl in 1979, S. 171-193.

HANUSCH, Horst u.a.: Verteilung öffentlicher Realtransfers auf Empfängergruppen in der Bundesrepublik Deutschland, Schriften zum Bericht der Transfer-Enquête-Kommission "Das Transfersystem in der Bundesrepublik Deutschland", Bde. 3.1 und 3.2, Stuttgart u.a. 1982.

HAX, Karl: Die öffentliche Unternehmung in der Marktwirtschaft, in: Finanzarchiv, N.F., Bd. 27 (1968), S. 37-48.

HEAD, John G.: Public Goods and Public Policy, in: Public Finance, Bd. 17 (1962), S. 197-219.

-: On Merit Goods, in: Finanzarchiv, N.F., Bd. 25 (1966), S. 1-29.

-: The Theory of Public Goods, in: Rivista di Diritto Finanziario e Scienca delle Finanze, Bd. 27 (1968), S. 209-236.

-: Merit Goods Revisited, in: Finanzarchiv, N.F., Bd. 28 (1969), S. 214-225.

-: Externality and Public Policy, in: Rivista di diritto Finanziario e Scienca delle Finanze, Bd. 28 (1969), S. 383-414.

HEAD, John G./SHOUP, Carl S.: Public Goods, Private Goods, and Ambiguous Goods, in: Economic Journal, Bd. 79 (1969), S. 567-572. 
HEAD, John G./SHOUP, Carl S.: Public, Private, and Ambiguous Goods Reconsidered, in: Public Finance, Bd. 28 (1973), S. 384-392.

HEDTKAMP, Günter: Bestimmungsgründe für Umfang und Struktur der öffentlichen Ausgaben, in: Beiträge zur Theorie der öffentlichen Ausgaben, hrsg. von H. Timm und H. Haller, Schriften des Vereins für Socialpolitik, N.F., Bd. 47, Berlin 1967, S. 66-103.

HENKE, Klaus-Dirk: Die mangelnde Aussagekraft der öffentlichen Finanzen als Indikator raumwirksamer Staatstätigkeit, in: Jahrbuch für Sozialwissenschaft, Bd. 25 (1974), S. 393-407.

HERDER-DORNEICH, Philipp: Politisches Modell zur Wirtschaftstheorie, Freiburg 1959. (Eine Teilauflage dieses Buches ist unter dem Pseudonym Fred 0 . Harding erschienen).

HERMANN, Friedrich Benedikt Wilhelm. von: Staatswirthschaftliche Untersuchungen, 2. Auf1., München 1870.

HERMANN, Walter: Intermediäre Finanzgewalten, Jena 1936.

HESSE, Günter: Staatsaufgaben. Zur Theorie der Legitimation und Identifikation staatlicher Aufgaben, Baden-Baden 1979.

HEYEN, Erk Volkmar: Das staatstheoretische und rechtstheoretische Problem des Beliehenen, Berlin 1973.

HILLMANN, Reinhard/WERSEBE, Hilmar von: Der Stand der Diskussion um eine Theorie der öffentlichen Aufgaben, in: Reform kommunaler Aufgaben, Studien zur Kommunalpolitik, Bd. 19, Bonn 1978, S. 65-84.

HOCHMAN, Harold M./RODGERS, James D.: Pareto Optimal Redistribution, in: The American Economic Review, Bd. 59 (1969), S. 542-557.

HOTZ, Beat: Politik zwischen Staat und Wirtschaft, Diessenhofen 1979.

HUBER, Ernst Rudolf: Wirtschaftsverwaltungsrecht, Bd. 1, 2. Aufl., Tübingen 1953.

HUMBOLDT, Wilhelm von: Ideen zu einem Versuch, die Grenzen der Wirksamkeit des Staates zu bestimmen, Berlin 1841.

INSTITUT für angewandtes Wirtschaftsrecht e.V.: Privatisierung der Risiken? Für eine Entstaatlichung nach wirtschaftlichen Grundsätzen, IaW-Schriften 2, Muinchen 0.J.

JAEGER, Klaus: Steuern, Entschädigungen und das Coase-Theorem, in: Jahrbuch für Sozialwissenschaft, Bd. 26 (1975), S. 211-227.

JANN, Werner: Kategorien der Policy-Forschung, Speyerer Arbeitshefte 37, Speyer 1981.

JECHT, Hans: Unternehmen und Verwaltungsorganisation, in: Verwaltung mit Unternehmen, hrsg. von H. Bülck u.a., Baden-Baden 1968, S. 65-87. 
JECHT, Horst: Wesen und Formen der Finanzwissenschaft. Umrisse einer Finanzwirtschaftslehre und Finanzsoziologie, Jena 1928.

JOACHIMSEN, Reimut: Ansatzpunkte der Wohlstandsökonomik, Tübingen 1969.

JOHANSEN, Leif: Public Economics, Amsterdam und London 1971.

JONSSON, Ernst: On the Rationing of Public Products (Services) by Queuing vis-a-vis Rationing by Price, in: Public Finance, 36. Ja. (1981), s. $430-438$.

KÁSTLI, René/SAURER, Peter: Aufgaben und Funktionsweise des Staates aus ökonomischer Sicht, in: Schweizerische Zeitschrift für Volkswirtschaft und Statistik, 114. Jg. (1978), S. 49-67.

KAHN, Alfred E.: The Economics of Regulation: Principles and Institutions, Vol. 1 und Vol. 2, New York u.a. 1970 und 1971.

KANTZENBACH, Erhard: Die Funktionsfähigkeit des Wettbewerbs, Göttingen 1966.

KANTZENBACH, Erhard/KALLFASS, Hermann H.: Das Konzept des funktionsfähigen

Wettbewerbs - workable competition -, in: Handbuch des Wettbewerbs, hrsg. von H. Cox, U. Jens und K. Markert, München 1981, S. 103-127.

KATTERLE, Siegfried: Wohlfahrtsökonomik und Theorie der Staatswirtschaft, in: Finanzarchiv, N.F., Bd. $30(1971 / 72)$, S. 14-26.

KAUFER, Erich: Theorie der öffentlichen Regulierung, Muinchen 1981.

KEHRLE, Karl: Rationalität und Effektivität des Wirtschaftspolitischen Trägersystems. Grundlagen der Verteilung wirtschaftspolitischer Entscheidungsbefugnisse, Frankfurt/M. 1980.

KEYNES, John M.: Das Ende des Laissez-Faire, München und Leipzig 1926.

KEYNES, John Neville: The Scope and Method of Political Economy, 4. Aufl., London, New York 1955 (1891).

KIRBERGER, Wolfgang: Staatsentlastung durch private Verbände. Die finanzpolitische Bedeutung der Mitwirkung privater Verbände bei der Erfüllung öffentlicher Aufgaben, Schriften zur öffentlichen Verwaltung und öffentlichen Wirtschaft, Bd. 30, Baden-Baden 1978.

KIRCHHOF, Paul: Der Begriff der hoheitlichen Befugnisse in Artikel 33 Absatz IV des Grundgesetzes, Diss., München 1968.

KLEIN, Hans H.: Zum Begriff der öffentlichen Aufgabe, in: Die Offentliche Verwaltung, 18. Jg. (1965), S. 755-759.

-: Die Teilnahme des Staates am wirtschaftlichen Wettbewerb, Stuttgart 1968.

KLEINSTEUBER, Hans J.: Art. Offentliche Bindung, in: Wörterbuch zur politischen Ökonomie, hrsg. von G. von Eynern und C. Böhret, 2. Aufl., Opladen 1977, S. 299-303. 
KNAPPE, Eckhard: Einkommensumverteilung in der Demokratie, Freiburg i.Br. 1980.

KNIEPS, Günter/MüLLER, Jürgen/WEIZSACKER, Carl C. von: Die Rolle des Wettbewerbs im Fernmeldebereich, Baden-Baden 1981.

KóLBLE, Josef: Entwicklung der Bundesaufgaben und ihrer Finanzierung im Hinblick auf das Grundgesetz, in: Entwicklung der Aufgaben und Ausgaben von Bund, Ländern und Gemeinden, Schriftenreihe der Hochschule Speyer, Bd. 47, Berl in 1971, S. 41-75.

KOSTERS, Rudolf: Die Regulierung externer Effekte, Tübingen 1979.

KOHN, Leopold: Probleme der Kollektivguiterallokation, Zürich 1973.

KOMMISSION für wirtschaftlichen und sozialen Wandel: Wirtschaftlicher und sozialer Wandel in der Bundesrepublik Deutschland, Gutachten der Kommission, Göttingen 1977.

KORTZFLEISCH, Gert von: Privater Wohlstand - offentliche Armut? Anmerkungen eines Systemanalytikers, in: Der Staatssektor in der sozialen Marktwirtschaft, hrsg. von D. Duwendag, Schriftenreihe der Hochschule Speyer, Bd. 59, Berlin 1976, S. 168-175.

KOSIOL, Erich: Organisation der Unternehmung, Wiesbaden 1962.

KRAUSE, Alfred: Privater Wohlstand - offentliche Armut?, in: Der Staatssektor in der sozialen Marktwirtschaft, hrsg. von D. Duwendag, Schriftenreihe der Hochschule Speyer, Bd. 59, Berlin 1976, S. 141-148.

KRAUSE-JUNK, Gerold: Probleme der Berechnung und Schätzung öffentlicher Ausgaben, in: Publ ic Finance, 27. Jg. (1972), S. 127-144.

-: Zur Theorie des distributiven Marktversagens, in: Offentliche Finanzwirtschaft und Verteilung II, hrsg. von W. Albers, Schriften des Vereins für Socialpolitik, N.F., Bd. 75/II, Berlin 1974, S. 33-67.

-: Abriß der Theorie von den öffentlichen Gütern, in: Handbuch der Finanzwissenschaft, Bd. 1, 3. Auf1., Tübingen 1977, S. 687-711.

KRAUTZBERGER, Michael: Die Erfüllung öffentlicher Aufgaben durch Private. Zum Begriff des staatlichen Bereichs, Berlin 1971.

KRUPP, Hans-Jürgen: Theorie der personellen Einkommensverteilung, Berlin 1968.

KüLP, Bernhard: Art. Wohlfahrtsökonomie I: Grundlagen, in: Handwörterbuch der Wirtschaftswissenschaft, 9. Bd., Tübingen 1982, S. 469-486.

KUX, Wilhelm: Ansätze zur Privatisierung öffentlicher Aufgaben, Institut für Kommunalwissenschaften der Konrad-Adenauer-Stiftung, St. Augustin 1976. 
LAUSCHMANN, Elisabeth: Grundlagen, Grenzen und Entwicklungsmöglichkeiten der Welfare Economics, in: Probleme der normativen okonomik und der wirtschaftlichen Beratung, hrsg. von E. von Beckerath und H. Giersch, Schriften des Vereins für Socialpolitik, N.F., Bd. 29, Berl in 1963, S. $99-128$.

LAUX, Eberhard: Aufgabenentwicklung und Personalbedarf in der öffentlichen Verwaltung, in: Die offentliche Verwaltung, 32. Jg. (1979), S. 729-737.

LEISNER, Walter: Werbefernsehen und öffentliches Recht, Berlin 1967.

-: Privatinteressen als öffentliches Interesse, in: Die offentliche Verwaltung, 23. Jg. (1970), S. 217-223.

LEPELMEIER, Dirk: Soziale Sicherung und Parafiskalität. Zur Einkommensumverteilungsproblematik im Bereich der Sozialversicherung, Frankfurt/M. 1979.

LINDBLOM, Charles E.: Inkrementalismus: die Lehre vom "Sich-Durchwursteln", in: Wohlfahrtsstaat und Massenloyalität, hrsg. von W.-D. Narr und C. Offe, Köln 1975, S. 161-177.

-: Jenseits von Markt und Staat. Eine Kritik der politischen und ökonomischen Systeme, Stuttgart 1980 (dt. Übersetzung von Politics and Markets. The World's Political-Economic System, New York 1977).

LIPSEY, Richard G./LANCASTER, Kelvin: The General Theory of Second Best, in: Review of Economic Studies, 24. Jg. (1956/57), S. 11-32.

LITTLE, I. M. D.: A Critique of Welfare Economics, 2. Auf1., Oxford 1957 (1. Auf1. 1950).

LITTLECHILD, S.C.: Misleading Calculations of the Social Costs of Monopoly Power, in: The Economic Journal, 91. Jg. (1981), S. 348-363.

LITTMANN, Konrad: Zunehmende Staatstätigkeit und wirtschaftliche Entwicklung, Köln und Opladen 1957.

--: Strukturen und Entwicklungen der staatlichen Aktivität in der Bundesrepublik Deutschland 1950-1970, in: Strukturwandlungen einer wachsenden Wirtschaft, hrsg. von F. Neumark, Schriften des Vereins für Socialpolitik, N.F., Bd. 30/II, Berlin 1964, S. 779-834.

-: Umweltbelastung - Sozialökonomische Gegenkonzepte, Göttingen 1974.

-: Definition und Entwicklung der Staatsquote, Göttingen 1975.

—: Die Chancen staatlicher Innovationslenkung, Göttingen 1975.

--: Ausgaben, öffentliche, II: Die "Gesetze" ihrer langfristigen Entwicklung, in: Handwörterbuch der Wirtschaftswissenschaft Bd. 1, StuttgartTüb ingen-Göttingen 1977, S. 349-363.

-: Problemstellung und Methoden der heutigen Finanzwissenschaft, in: Handbuch der Finanzwissenschaft, Bd. 1, 3. Auf1., Tübingen 1977, S. 99-120. 
LOESCH, Achim von: Die gemeinwirtschaftliche Unternehmung, Köln 1977.

LOSCH, Dieter: Zur Ideologiekritik des traditionellen bipolaren Ordnungsdenkens, in: Hamburger Jahrbuch für Wirtschafts- und Gesellschaftspolitik, 20. Jg. (1975), S. 87-109.

LUHMANN, Niklas: Theorie der Verwaltungswissenschaft, Bestandsaufnahme und Entwurf, Köln und Berlin 1966.

MACHLUP, Fritz: Art. Monopol, in: HdSW, Bd. 7, Tübingen 1961, S. 427-452.

MACKSCHEIDT, Klaus: Zur Theorie des optimalen Budgets, Tübingen 1973.

-: Entstehung öffentlicher Aufgaben und öffentlicher Güter, in: Reform kommunaler Aufgaben, Institut für Kommunalwissenschaften, Studien zur Kommunalpolitik, Bd. 19, Bonn 1978, S. 47-64.

-: Zur Kritik einiger Rechtfertigungsargumente für staatliche Interventionen auf dem Wohnungsmarkt, in: Zeitschrift für Wirtschafts- und Sozialwissenschaften, 102. Jg. (1982), S. 113-134.

MACKSCHEIDT, Klaus/STEINHAUSEN, Jörg: Finanzpolitik II. Grundfragen versorgungspolitischer Eingriffe, Tübingen 1977.

MADDING, Erhard: Aufgaben der öffentlichen Verwaltung, in: Die Verwaltung, Bd. 6 (1973), S. 257-282.

-: Aufgaben als Reformthema, in: Reform kommunaler Aufgaben, Institut für Kommunalwissenschaften, Studien zur Kommunalpolitik, Bd. 19, Bonn 1978, S. $1-46$.

MANN, Fritz Karl: Die intermediären Finanzgewalten und ihr Einfluß auf Deutschlands finanzielle Belastung, in: Jahrbücher für Nationalökonomie und Statistik, Bd. 129 (1928), S. 219-237.

-: Die Staatswirtschaft unserer Zeit, Jena 1930.

MARJOLIS, Julius: A Comment on the Pure Theory of Public Expenditure, in: The Review of Economics and Statistics, Bd. 37 (1955), S. 347-349.

MARX, Karl/ENGELS, Friedrich: Manifest der Kommunistischen Partei, Berlin 1945.

MAUNZ, Theodor: Der öffentliche Charakter der kirchlichen Aufgaben, in: Festschrift für E. Forsthoff zum 70. Geburtstag, hrsg. von R. Schnur, 2. Auf1., München 1974, S. 229-240.

MAYNTZ, Renate: Soziologie der öffentlichen Verwaltung, Heidelbero und Karlsruhe 1978.

-: Sachverständigenanhörung zu Ursachen einer Bürokratisierung in der öffentlichen Verwaltung sowie zu ausgewählten Vorhaben zur Verbesserung des Verhältnisses von Bürger und Verwaltung am 19. und 20. Juni 1980 in Bonn, Teil C: Wissenschaftliche Auswertung Gesetzgebung und Bürokratisierung, Bonn 1980 . 
McLliRE, Charles E.: Merit Wants: a Normatively Empty Box, in: Finanzarchiv, N.F., Bd. 27 (1968), S. 375-483.

MEADE, James G.: External Economies and Diseconomies in a Competitive Situation, in: Economic Journal, Bd. 62 (1952), S. 54-67.

MICHAELIS, Rüdiger: Der Beliehene. Ein Beitrag zur Verflechtung von öffentlichem und privatem Recht, Diss., Münster 1969.

MISHAN, E. J.: A Survey of Welfare Economics 1939-59, in: Economic Journal, Vol. 70 (1960), S. 197-265.

-: The Postware Literature on Externalities: An Interpretative Essay, in: The Journal of Economic Literature, Bd. 9 (1971), S. 1-28.

MITNICK, Barry M.: The Political Economy of Regulation. Creating, Designing, and Removing Regulatory Forms, New York 1980.

MORGENSTERN, Oskar: Descriptive, Predictive, and Normative Theory, in: Kyklos, Bd. 25 (1972), S. 699-714.

MORSS, Elliott R.: Some Thoughts on the Determinants of State and Local Expenditures, in: National Tax Journal, Bd. 19 (1966), S. 95-103.

MÜLLER, Jürgen/VOGELSANG, Ingo: Ist eine Effizienzsteigerung der öffentlichen Verwaltung durch Anwendung des Instrumentariums der amerikanischen "Public Utility Regulation" möglich?, in: Neuere Entwicklungen in den Wirtschaftswissenschaften, hrsg. von E. Helmstädter, Schriften des Vereins für Socialpolitik, N.F., Bd. 98, Berlin 1978, S. 775-793

-: Staatliche Regulierung, Baden-Baden 1979.

-: Das amerikanische Konzept staatlicher Regulierung im Vergleich mit öffentlichen Unternehmen in der Bundesrepublik Deutschland, in: Zeitschrift für öffentliche und gemeinwirtschaftliche Unternehmen, 2. Jg. (1979), S. 115126.

MULLER-THOMA, Frank: Der halbstaatliche Verein. Eine Organisationserscheinung der institutionalisierten Durchdringung von Staat und Gesellschaft, Berlin 1974.

MÜNCH, Klaus N.: Kollektive Güter und Gebüinren. Elemente einer Gebührentheorie für Kollektivgüter, Göttingen 1976.

MUSGRAVE, Richard A.: The Theory of Public Finance, New York, Toronto, London 1959, in deutscher übersetzung erschienen unter dem Titel: Finanztheorie, 2. Aufl., Tübingen 1969.

-: Provision for Social Goods, in: Public Economics. An Analysis of Public Production and Consumption and their Relations to the Private Sector, hrsg. v. J. Margolis und H. Guitton, London, Melbourne, Toronto, New York 1969, S. 124-144. 
MUSGRAVE, Richard A.: Allocation, Distribution, and the Theory of Public Finance, in: H. Haller u.a. (Hrsg.): Theorie und Praxis des finanzpolitischen Interventionismus, Fritz Neumark zum 70. Geburtstag, Tübingen 1970, S. 29-45.

-: Provision for Social Goods in the Market System, in: Public Finance, Bd. 26 (1971), S. 304-320.

MUSGRAVE, Richard A./PEACOCK, Alan T. (Hrsg.): Classics in the Theory of Public Finance, 4. Aufl., New York 1967.

NACHTKAMP, Hans Heinrich: Idealtypen von Fiskalsystemen. Ein Versuch, in: Finanzsysteme: Ideal- und Realtypen - Gesundheitswesen und Hochschulbildung, hrsg. von K. Häuser, Berlin 1983, S. 11-53.

NARR, Wolf-Dieter: Theoriebegriffe der Systemtheorie, 3. Aufl., Stuttgart, Berlin, Köln, Mainz 1972, S. 151.

OAKLAND, William H.: Congestion, Public Goods and Welfare, in: Journal of Public Economics Bd. 1 (1972), S. 339-357.

OBENDORFER, Dieter: Zur Typologie der Verbände, in: Staatsführung, Verbandsmacht und innere Souveränität, hrsg. von K. H. Biedenkopf und R. von Voss, Stuttgart 1977, S. 20-24.

OETTLE, Karl: über den Charakter öffentlich-wirtschaftlicher Zielsetzungen, in: Zeitschrift für betriebswirtschaftliche Forschung, N.F., 16. Jg. (1966), S. 241-259.

-: Die ökonomische Bedeutung der Rechtsform öffentlicher Betriebe, in: Archiv für öffentliche und freigemeinnützige Unternehmen, 8. Jg. (1967), S. 193226.

OLSON jr., Mancur: The Logic of Collective Action, Public Goods and the Theory of Groups, Cambridge, Mass. 1965.

OSSENBUHL, Fritz: Die Erfüllung von Verwaltungsaufgaben durch Private, in: Veröffentlichungen der Vereinigung Deutscher Staatsrechtslehrer, Bd. 29 Berlin 1971, S. 137-236.

OTT, Alfred E.: Grundzüge der Preistheorie, 2. Auf1., Göttingen 1970.

PETERS, Hans: Offentliche und staatliche Aufgaben, in: Festschrift für Hans Carl Nipperdey zum 70. Geburtstag, Bd. 2, München und Berlin 1965, S. 877-895.

PICHT, Christine: Möglichkeiten einer verstärkten Verlagerung öffentlicher Dienste auf freiberuflich Tätige, Berlin 1984.

POSNER, Richard A.: The Social Costs of Monopoly and Regulation, in: Journal of Political Economy, 83. Jg. (1975), S. 807-827.

PRYOR, Frederic L.: Elements of a Positive Theory of Public Expenditure, in: Finanzarchiv, N.F., Bd. 26 (1967), S. 405-430. 
PÜTTNER, Günter: Die öffentlichen Unternehmen, Bad Homburg V.d.H. 1969.

PUTZ, Theodor: Das "Gemeinwohl" als Begriff der theoretischen Wirtschaftspolitik, in: Zeitschrift für Nationalökonomie, 26. Jg. (1966), S. 260267.

PULSIPHER, Allan G.: The Properties and Relevancy of Merit Goods, in: Finanzarchiv, N.F., Bd. $30(1971 / 72)$, S. 266-286.

RAABE, Karl-Heinz: Projektionen eines "Korridors für den Staatssektor", in: Der Staatssektor in der sozialen Marktwirtschaft, hrsg. von D. Duwendag, Schriftenreihe der Hochschule Speyer, Bd. 59, Berlin 1976, S. 37-59.

DER RAT VON SACHVERSTANNDIGEN FÜR UMWELTFRAGEN: Umweltgutachten 1974, BTDrucksache $7 / 2802$ vom 14.11.1974.

-: Umweltgutachten 1978, Stuttgart und Mainz 1978.

RECKTENWALD, Horst C.: Staatsausgaben in säkularer Sicht. Versuch einer Beschreibung, in: Theorie und Praxis des finanzpolitischen Interventionismus. F. Neumark zum 75. Geburtstag, hrsg. von H. Haller u.a., Tübingen 1970 , S. 407-430.

REINERMANN, Heinrich: Programmbudgets in Regierung und Verwaltung, BadenBaden 1975.

RIEGER, Wilhelm: Einführung in die Privatwirtschaftslehre, 3. Aufl:, Erlangen 1964 (erste Auf1. 1928).

RITTIG, Gisbert: Die Definitionen des Terminologie-Ausschusses der Gesellschaft zur Förderung der öffentlichen Wirtschaft, in: Archiv für öffentliche und freigemeinwirtschaftliche Unternehmen, Bd. 1 (1954), S. 214224.

ROPPE, Burkhardt: Zur Frage nach den Trägern der Wirtschaftspolitik, in: Zeitschrift für die gesamte Staatswissenschaft, Bd. 124 (1968), S. 741-762.

ROSE, Richard: On the Priorities of Government: A Developmental Analysis of Public Policies, in: European Journal of Political Research, 4 (1976), S. $247-289$.

SACHVERSTANDIGENRAT zur Begutachtung der gesamtwirtschaftlichen Entwicklung, Jahresgutachten 1975/76, Stuttgart, Mainz 1975.

SAMUELSON, Paul A.: The Pure Theory of Public Expenditure, in: Review of Economics and Statistics, Bd. 36 (1954), S. 387-389.

-: Diagrammatic Exposition of a Theory of Public Expenditure, in: Review of Economics and Statistics, Bd. 37 (1955), S. 350-356.

--: Aspects of Public Expenditure Theories, in: Review of Economics and Statistics, Bd. 40 (1958), S. 332-338. 
SAMUELSON, Paul A.: Pure Theory of Expenditure and Taxation, in: Public Economics. An Analysis of Public Production and Consumption and their Relations of the Private Sector, hrsg. von J. Margol is und H. Guitton, London, Melbourne, Toronto, New York 1969, S. 98-123.

SCHARPF, Fritz W.: Komplexität als Schranke der politischen Planung, in: Politische Vierteljahresschrift, Sonderheft 4 (1972), S. 168-192.

-: Planung als politischer Prozeß, Frankfurt/M. 1973.

SCHLIEPER, Ulrich: Art. Wohlfahrtsökonomik II: Theorie des Zweitbesten, in: Handwörterbuch der Wirtschaftswissenschaft, 9. Bd., Tübingen 1982 , S. $486-493$.

SCHMELCHER, Ernst: Art. Gemischtwirtschaftliche Unternehmen, in: Handwörterbuch der Staatswissenschaften, 4. Bd., Jena 1927, S. 846-852.

SCHMIDT, Kurt: Zur Geschichte der Lehre von den Kollektivbedürfnissen, in: Systeme und Methoden in den Wirtschafts- und Sozialwissenschaften. E. von Beckerath zum 75. Geburtstag, hrsg. von N. Kloten U.a., Tübingen 1964 , S. 335-362.

-: Zu einigen Theorien über die relative Ausdehnung der öffentlichen Ausgaben, in: Finanzarchiv, N.F., Bd. 24 (1965), S. 193-208.

-: Kollektivbedürnisse und Staatstätigkeit, in: Theorie und Praxis des finanzpolitischen Interventionismus. Fritz Neumark zum 70. Geburtstag, hrsg. von H. Haller u.a., Tübingen 1970, S. 3-27.

SCHMOLLDER, Günter: Das Selbstbild der Verbände, Schriften des Vereins für Socialpolitik, N.F., Bd. 38, Berlin 1965.

-: Finanzpolitik, 3. Aufl., Berlin, Heidelberg, New York 1970.

SCHNETTLER, Albert: Offentliche Betriebe, Essen 1956.

SCHOLZ, Rupert: Grenzen staatlicher Aktivität unter der grundgesetzlichen Wirtschaftsverfassung, in: Der Staatssektor in der sozialen Marktwirtschaft, hrsg. von D. Duwendag, Schriftenreihe der Hochschule Speyer, Bd. 59, Berlin 1976, S. 113-133.

SCHUBERT, Glendon: The Public Interest. A Critique of the Theory of a Political Concept, Clencoe, I11. 1960.

SCHULTZE, Charles L.: The Public Use of Private Interest, Washington D.C. 1977.

SCHUMPETER, Josef A.: Kapitalismus, Sozialismus und Demokratie, Bern 1946.

SCHUPPERT, Gunnar F., Die öffentliche Aufgabe als Schlüsselbegriff der Verwaltungswissenschaft, in: Verwaltungsarchiv, Bd. 71 (1980), S. 309-344.

-: Die Erfüllung öffentlicher Aufgaben durch verselbständigte Verwaltungseinheiten, Göttingen 1981. 
SCHWARTING, Gunnar: Positive Theorien der Staatsausgaben, in: Schweizerische Ze-itschrift für Volkswirtschaft und Statistik, 116. Jg. (1980), S. 67-91.

SHUBIK, Martin: On Different Methods for Allocating Resources, in: Kyklos, 23. Jg. (1970), S. 332-337.

SIEBERT, Horst: Analyse der Instrumente der Umweltpolitik, Göttingen 1976.

-: Neuere Entwicklungen in der ökonomischen Analyse des Umweltschutzes, in: Umweltökonomik, hrsg. von H. Möller, Königstein/Ts. 1982, S. 267283.

SINGER, Neil M.: Public Microeconomics, Boston 1972.

SMEKAL, Christian: Die Finanzwirtschaft intermediärer Gruppen, Innsbruck 1969.

SMITH, Adam: Der Wohlstand der Nationen. Eine Untersuchung seiner Natur und seiner Ursachen. Aus dem Englischen übertragen und mit einer Würdigung von H. C. Recktenwald, München 1974.

SMOLENSKY, Eugene/TIDSMAN, T. Nicolaus/NICHOLS, Donald: Waiting Time as a Congestion Charge, in: G. Mushkin (ed.): Public Prices for Public Products, Washington D.C. 1972, S. 95-108.

SOHMEN, Egon: Grundlagen, Grenzen und Entwicklungsmöglichkeiten der Welfare Economics, in: Probleme der normativen okonomik und der wirtschaftlichen Beratung, hrsg. von E. von Beckerath und H. Giersch, Schriften des Vereins für Socialpolitik, N.F., Bd. 29, Berlin 1963, S. 69-98.

-: Allokationstheorie und Wirtschaftspolitik, Tübingen 1976.

STACKELBERG, Heinrich von: Grundlagen der theoretischen Volkswirtschaftslehre, 2. Auf1., Tübingen und Zürich 1951.

STEINER, Udo: Offentliche Verwaltung durch Private, Hamburg 1975.

STOHLER, Jacques: Zur Methode und Technik der Cost-Benefit-Analyse, in: Kyklos, 20. Jg. (1967), S. 218-245.

STREETEN, Paul: Programs and Prognoses, in: Quarterly Journal of Economics, Bd. 68 (1954), S. 355-376.

STUDIENGRUPPE für Sozialforschung e.V.: Gutachten zu den Bedingungen und Möglichkeiten einer Privatisierung öffentlicher Dienstleistungen durch übertragung an gebührennehmende Freie Berufe, München 1980.

THIEL, Eberhard: Zielgerechte Veränderung der Wahrnehmung von Staatsaufgaben, in: Hamburger Jahrbuch für Wirtschafts- und Gesellschaftspolitik, 22. Jg. (1977), S. 55-71.

THIEMEYER, Theo: Wirtschaftslehre öffentlicher Betriebe, Reinbek bei Hamburg 1975.

THUROW, Lester C.: The Income Distribution as a Pure Public Good, in: Quarterly Journal of Economics, Bd. 85 (1971), S. 327-336. 
TIEPELMANN, Klaus: Parafiski, in: WiSU, 4. Jg. (1975), S. 295-300.

TOBIN, James A.: A Survey of the Theory of Rationing, in: Econometrica, 20. Jg. (1952), S. 521-553.

TOMUSCHAT, Christian: Güterverteilung als rechtliches Problem, in: Der Staat, 13. Jg. (1973), S. 433-466.

TUCHTFELDT, Egon: Wirtschaftspolitik und Verbände, in: Hamburger Jahrbuch für Wirtschafts- und Gesellschaftspolitik, 1. Jg. (1956), S. 72-84.

-: Ober die Staatsfunktionen bei Adam Smith, in: Ordo, 27. Jg. (1976), S. $29-45$.

VAN DER BELLEN, Alexander: Offentliche Unternehmen zwischen Markt und Staat, Köln 1977.

VICKREY, William S.: Decreasing Cost, Publicy Administered Prices, and Economic Efficiency, in: The Analys is and Evaluation of Public Expenditures: The PPB System, A Compendium of Papers submitted to the Subcommittee on Economic in Government of the Joint Economic Committee Congress of the United States, Vol. 1, Washington 1969, S. 119-148.

VOGT, Fritz: Die Bedeutung der Rechtsform für die Einflußmöglichkeiten der Gemeinden auf die Zielbildung kommunaler Versorgungsbetriebe, dargestellt am Beispiel des Eigenbetriebes und der Eigengesellschaft, Diss., Mannheim 1970.

WAGENER, Frido: Typen der verselbständigten Erfüllung öffentlicher Aufgaben, in: Verselbständigung von Verwaltungsträgern, hrsg. von F. Wagener, Schriften der Deutschen Sektion des Internationalen Instituts für Verwaltungswissenschaften, Bd. 1, Bonn 1976, S. 31-50.

- : Organisation der öffentlichen Verwaltung, in: Handwörterbuch der Organisation, 2. Auf1., Stuttgart 1980, Sp. 1407-1417.

WAGNER, Adolph: Grundlegung der politischen ókonomie, Theil 1: Grundlagen der Volkswirtschaft, 3. Auf1., Leipzig-Heidelberg 1893.

WEGEHENKEL, Lothar: Coase-Theorem und Marktsystem, Tübingen 1980.

-: Gleichgewicht, Transaktionskosten und Evolution, Tübingen 1981.

WEHNER, Georg: Privater Wohlstand - Offentliche Armut?, in: Der Staatssektor in der sozialen Marktwirtschaft, hrsg. von D. Duwendag, Schriftenreihe der Hochschule Speyer, Bd. 59, Berlin 1976, S. 160-167.

WEIDENBAUM, Murray L.: The High Cost of Government Regulation, in: Challenge, Nov./Dec. 1979, S. 32-39. 
WEISSER, Gerhard: Das Problem der systematischen Verknüpfung von Normen und von Aussagen der positiven Okonomik in grundsätzlicher Betrachtung, erläutert anhand des Programms einer sozialwissenschaftlichen Grunddisziplin aus Empfehlungen und Warnungen, in: Probleme der normativen Okonomik und der wirtschaftlichen Beratung, hrsg. von E. von Beckerath und $H$. Giersch, Schriften des Vereins für Socialpolitik, N.F., Bd. 29, Berlin 1962, S. 16-31.

WEIZSÄCKER, Richard von: Leistet der Markt die optimale intertemporale Allokation der Ressourcen?, in: Erschöpfbare Ressourcen, hrsg. von H. Siebert, Schriften des Vereins für Socialpolitik, Bd. 108, Berlin 1980, S. 795-814.

-: Staatliche Regulierung - positive und normative Theorie, in: Schweizerische Zeitschrift für Volkswirtschaft und Statistik, 118. Jg. (1982), S. 325-343.

WILLE, Eberhard/GLÄSER, Martin: Staatsaufgaben bei Adam Smith - eine Würdigung unter Allokationsaspekten, in: Ordo, 28. Jg. (1977), S. 34-77.

WILLE, Eberhard: Art. Offentlicher Haushalt IV: Finanz- und Aufgabenplanung, in: Handwörterbuch der Wirtschaftswissenschaft (HdWW), Bd. 5, Stuttgart u.a. 1980, S. 591-620.

WISSENSCHAFTLICHER BEIRAT beim Bundesministerium der Finanzen: Gutachten zur Finanzierung eines höheren Staatsanteils am Sozialprodukt vom 3.6.1972, in: Wissenschaftlicher Beirat beim BMF. Entschließungen, Stellungnahmen und Gutachten 1949-1973, hrsg. vom Bundesministerium der Finanzen, Tübingen 1974 , S. 535-571.

-: Zur Lage und Entwicklung der Staatsfinanzen in der Bundesrepublik Deutschland, Bulletin der Bundesregierung Nr. 103 vom 16.8.1975, S. 1001-1016.

-: Gutachten zur Aussagefähigkeit staatswirtschaftlicher Quoten, Bulletin der Bundesregierung, $\mathrm{Nr}$. 90 vom 30.7.1976, S. 849-862.

WISSENSCHAFTLICHER BEIRAT für Familienfragen beim BMJFG: Familie und Wohnen, Analysen und Empfehlungen zur Wohnungsversorgung der Familien und zur Förderung familiengerechten Wohnens in der Bundesrepublik Deutschland, Schriftenreihe des Bundesministers, Bd. 20, Stuttgart 1975.

WISSENSCHAFTLICHER BEIRAT beim Bundesministerium der Wirtschaft: Kosten und Preise öffentlicher Unternehmen, Bulletin der Bundesregierung Nr. 4 vom 8.1.1976, S. 36-48.

WOHE, Günter: Einführung in die allgeme ine Betriebswirtschaftslehre, 12. Auf1., München 1976.

WOLFF, Hans J./BACHOF, Otto: Verwaltungsrecht I, 9. Auf1., München 1974.

-: Verwaltungsrecht II, 4. Auf1., München 1976.

ZIMMERMANN, Horst: Die Ausgabenintensität der öffentlichen Aufgabenerfüllung, in: Finanzarchiv, N.F., Bd. 32 (1974), S. 1-20. 
ZIMMERMANN, Horst/HENKE, Klaus-Dirk: Finanzwissenschaft. Eine Einführung in die Lehre von der öffentlichen Finanzwirtschaft, München 1975.

ZINN, Karl Georg: Allgemeine Wirtschaftspolitik, 2. Auf1., Stuttgart, Berlin, Köln, Mainz 1974.

ZUMBOHL, Marcel: Privatisierung staatlicher Wirtschaftstätigkeit. Notwendigkeit und Möglichkeiten?, Zürich 1978. 


\section{FINANZWISSENSCHAFTLICHE SCHRIFTEN}

Band 1 Werner Steden: Finanzpolitik und Einkommensverteilung. Ein Wachstums- und Konjunkturmodell der Bundesrepublik Deutschland. 1979.

Band 2 Rainer Hagemann: Kommunale Finanzplanung im föderativen Staat. 1976.

Band 3 Klaus Scherer: Maßstäbe zur Beurteilung von konjunkturellen Wirkungen des öffentlichen Haushalts. 1977.

Band 4 Brita Steinbach: „Formula Flexibility” - Kritische Analyse und Vergleich mit diskretionärer Konjunkturpolitik. 1977.

Band 5 Hans-Georg Petersen: Personelle Einkommensbesteuerung und Inflation. Eine theoretisch-empirische Analyse der Lohn- und veranlagter, Einkommensteuer in der Bundesrepublik Deutschland. 1977.

Band 6 Friedemann Tetsch: Raumwirkungen des Finanzsystems der Bundesrepublik Deutschland. Eine Untersuchung der Auswirkungen der Finanzreform von 1969 auf die Einnahmenposition der untergeordneten Gebietskörperschaften und ihrer regionalpolitischen Zieladäquanz. 1978.

Band 7 Wilhelm Pfähler: Normative Theorie der fiskalischen Besteuerung. Ein methodologischer und theoretischer Beitrag zur Integration der normativen Besteuerungstheorie in der Wohlfahrtstheorie. 1978.

Band 8 Wolfgang Wiegard: Optimale Schattenpreise und Produktionsprogramme für öffentliche Unternehmen. Second-Best-Modelle im finanzwirtschaftlichen Staatsbereich. 1978.

Band 9 Hans P. Fischer: Die Finanzierung des Umweltschutzes im Rahmen einer rationalen Umweltpolitik. 1978.

Band 10 Rainer Paulenz: Der Einsatz finanzpolitischer Instrumente in der Forschungs- und Entwicklungspolitik. 1978.

Band 11 Hans-Joachim Hauser: Verteilungswirkungen der Staatsverschuldung. Eine kreislauftheoretische Inzidenzbetrachtung. 1979.

Band 12 Gunnar Schwarting: Kommunale Investitionen. Theoretische und empirische Untersuchungen der Bestimmungsgründe kommunaler Investitionstätigkeit in Nordrhein-Westfalen 1965-1972. 1979.

Band 13 Hans-Joachim Conrad: Stadt-Umland-Wanderung und Finanzwirtschaft der Kernstädte. Amerikanische Erfahrungen, grundsätzliche Zusammenhänge und eine Fallstudie für das Ballungsgebiet Frankfurt am Main. 1980.

Band 14 Cay Folkers: Vermögensverteilung und staatliche Aktivität. Zur Theorie distributiver Prozesse im Interventionsstaat. 1981.

Band 15 Helmut Fischer: US-amerikanische Exportförderung durch die DISC-Gesetzgebung. 1981.

Band 16 Günter Ott: Einkommensumverteilungen in der gesetzlichen Krankenversicherung. Eine quantitative Analyse. 1981.

Band 17 Johann Hermann von Oehsen: Optimale Besteuerung. (Optimal Taxation). 1982.

Band 18 Richard Kössler: Sozialversicherungsprinzip und Staatszuschüsse in der gesetzlichen Rentenversicherung. 1982.

Band 19 Hinrich Steffen: Zum Handlungs- und Entscheidungsspielraum der kommunalen Investitionspolitik in der Bundesrepublik Deutschland. 1983.

Band 20 Manfred Scheuer: Wirkungen einer Auslandsverschuldung des Staates bei flexiblen Wechselkursen. 1983.

Band 21 Christian Schiller: Staatsausgaben und crowding-out-Effekte. Zur Effizienz einer Finanzpolitik keynesianischer Provenienz. 1983. 
Band 22 Hannelore Weck: Schattenwirtschaft: Eine Möglichkeit zur Einschränkung der öffentlichen Verwaltung? Eine ökonomische Analyse. 1983.

Band 23 Wolfgang Schmitt: Steuern als Mittel der Einkommenspolitik. Eine Ergänzung der Stabilitätspolitik? 1984.

Band 24 Wolfgang Laux: Erhöhung staatswirtschaftlicher Effizienz durch budgetäre Selbstbeschränkung? Zur Idee einer verfassungmäßig verankerten Ausgabengrenze. 1984.

Band 25 Brita Steinbach-van der Veen: Steuerinzidenz. Methodologische Grundlagen und empirisch-statistische Probleme von Länderstudien. 1985.

Band 26 Albert Peters: Ökonomische Kriterien für eine Aufgabenverteilung in der Marktwirtschaft. Eine deskriptive und normative Betrachtung für den Allokationsbereich. 1985. 
Albert Peters - 978-3-631-75163-3

Downloaded from PubFactory at 01/11/2019 07:34:44AM

via free access 
Albert Peters - 978-3-631-75163-3

Downloaded from PubFactory at 01/11/2019 07:34:44AM

via free access 